\title{
ASSESSING AND ENRICHING THE CAGE ENVIRONMENT OF
}

LABORATORY RATS

\author{
A thesis \\ submitted in fulfilment \\ of the requirements for the Degree \\ of \\ Doctorate of Philosophy \\ in Psychology \\ at \\ Victoria University of Wellington \\ by \\ EMILY GAY PATTERSON-KANE
}

Victoria University of Wellington

1999 


\begin{abstract}
Public concern with the caging conditions of animals kept in laboratories led to research assessing the standard conditions of rats housed in New Zealand laboratories. A total of 113 rats were used in experiments of four basic types. The experiments presented in the first and second chapters assessed the behaviours of rats housed in enriched, standard and deprived conditions. The assessment procedures used were the emergence box, open field and Hebb William's maze as well as the T-maze and a range of operant procedures. The behaviour of rats housed in standard conditions for the emergence box, open field and maze were intermediate between the enriched and deprived rats, but more closely resembled that of the deprived rats. However, the deprived rats displayed no general cognitive deficits on procedures other than the Hebb William's maze, causing the validity of the maze in this context to be questioned. A more specific cognitive deficit relating to attention at the time of encoding was indicated. The thesis then moved from looking for behavioural damage to examining what conditions rats would prefer, and extending these findings using behavioural economics. The rats showed significant preferences for only a small number of cage modifications. They clearly preferred nesting boxes and shredded paper, and showed some preference for a larger group size of rats. The demand experiments demonstrated that the rats worked hardest for access to moderately sized environments with a group size of six. Therefore, the recommendation arising from the current study is that rats should be provided with nest boxes and paper, and provision should be made in the future for using cages suitable for groups of around six. There were also implications for the range of procedures used during the course of this investigation. The open field data suffers from an unstandardised procedure
\end{abstract}


that probably allows a range of confounding variables to come into effect, specifically changes in activity across time. The preference tests (T-maze and continuous access) gave broadly equivalent data although there were small systematic differences in the results between the two tests which suggest they should be used together in order to cancel out these biases. The demand procedure is the pre-eminent option from a theoretical point of view but the detail of the procedure is in need of some development. The best way to achieve this progress would be through more extensive applied use of behavioural economics. 


\section{ACKNOWLEDGEMENTS}

Thanks to my supervisors Dr. Maree Hunt and Dr. David Harper, and the rats without whom none of this would have been possible. I would like to acknowledge those involved in endowing and administering the Victoria University of Wellington Postgraduate Scholarship and the William Georgetti Scholarship. Money may not be everything, but it certainly makes a lot of things possible. Also the Royal Society and the Beaglehole fund that provided funding for conference presentations at the European Meeting for the Experimental Analysis of Behaviour (1997), The Behaviour Analysis conferences (1995 and 1996) and the NZPA and PGSA conferences (1998). Finally, thanks to Judi, David, Craig, Linton and Darrell for good advice, not always well received. 


\section{TABLE OF CONTENTS}

$\begin{array}{lc}\text { ABSTRACT } & \text { ii } \\ \text { ACKNOWLEDGEMENTS } & \text { iv } \\ \text { TABLE OF CONTENTS } & \text { v } \\ \text { LIST OF TABLES } & \text { viii } \\ \text { LIST OF FIGURES } & \text { ix } \\ \text { LIST OF APPENDICES } & \text { xii }\end{array}$

CHAPTER 1: Behavioural Effects of Enrichment 1

EXPERIMENT 1: Evaluating the Standard Caging Conditions 32

$\begin{array}{ll}\text { Method } & 35\end{array}$

$\begin{array}{ll}\text { Results } & 39\end{array}$

$\begin{array}{ll}\text { Discussion } & 44\end{array}$

CHAPTER 2: The Cognitive Deficit 53

EXPERIMENT 2.1: Memory $\quad 54$

$\begin{array}{ll}\text { Method } & 56\end{array}$

$\begin{array}{ll}\text { Results } & 59\end{array}$

$\begin{array}{ll}\text { Discussion } & 64\end{array}$

$\begin{array}{ll}\text { EXPERIMENT 2.2: Persistence } & 67\end{array}$

$\begin{array}{ll}\text { Method } & 70\end{array}$

$\begin{array}{ll}\text { Results } & 72\end{array}$

$\begin{array}{ll}\text { Discussion } & 78\end{array}$

$\begin{array}{ll}\text { EXPERIMENT 2.3: Learning } & 80\end{array}$ 
$\begin{array}{ll}\text { Method } & 83\end{array}$

$\begin{array}{ll}\text { Results } & 85\end{array}$

$\begin{array}{ll}\text { Discussion } & 90\end{array}$

$\begin{array}{ll}\text { EXPERIMENT } 2.4 & 96\end{array}$

$\begin{array}{ll}\text { Method } & 97\end{array}$

$\begin{array}{ll}\text { Results } & 98\end{array}$

$\begin{array}{ll}\text { Discussion } & 100\end{array}$

$\begin{array}{ll}\text { General Discussion } & 101\end{array}$

$\begin{array}{ll}\text { CHAPTER 3: Preference } & 105\end{array}$

EXPERIMENT 3.1: Three Studies on Rats' Preferences for Objects 114

$\begin{array}{ll}\text { Method } & 116\end{array}$

$\begin{array}{ll}\text { Results } & 119\end{array}$

$\begin{array}{ll}\text { Discussion } & 124\end{array}$

EXPERIMENT 3.2: Preference for the Dimensions of Enrichment 126

$\begin{array}{ll}\text { Method } & 127\end{array}$

$\begin{array}{ll}\text { Results } & 128\end{array}$

$\begin{array}{ll}\text { Discussion } & 128\end{array}$

$\begin{array}{ll}\text { EXPERIMENT 3.3: Preferences for Cage Furniture } & 133\end{array}$

Method 134

$\begin{array}{ll}\text { Results } & 135\end{array}$

$\begin{array}{ll}\text { Discussion } & 138\end{array}$

$\begin{array}{ll}\text { CHAPTER 4: Demand } & 140\end{array}$

EXPERIMENT 4.1: The Effect of Economy on Demand Intensity in Short

$\begin{array}{ll}\text { Sessions } & 152\end{array}$ 
Method

Results

Discussion

EXPERIMENT 4.2: Rat's Demand for the Dimensions of Enrichment

Method

Results

Discussion

EXPERIMENT 4.3: Demand for Different Sized Social Groups

Method

Results

Discussion 


\section{LIST OF TABLES}

TABLE

PAGE

1 Interventions that result in changes in open field activity levels

$2 K$ and $R o$ values from Herrnstein's hyperbolas fitted to the individual data of seven deprived and eight enriched rats

3 Mean choices for the intervention out of a total of 50

4 Individual ratings of commodities based on individual's last FR where a commodity was obtained

5 Individual ratings of commodities based on individual's pmax data

6 Inelasticity hierarchies based on pmax parameters 


\section{LIST OF FIGURES}

FIGURE

PAGE

1 Photographs of the emergence Box, open field and Hebb William's

maze

2 Latencies to emerge from the dark half of a box into the light half

3 Mean number of open field squares entered during the first two minutes

of the first session for deprived, standard, semi-enriched and enriched

rats

4 Mean open field squares entered in each minute of the first session

5 Open field squares entered as a function of consecutive minutes during

Sessions Two and Three

6 Hebb-Williams maze errors; total (whole bar), initial (filled portion) and repeated (white section) errors for deprived, standard, semi-enriched and enriched rats

7 Percent correct responses as a function of inter-trial delay, for rats from deprived and enriched caging

8 Proportion correct as a function of inter-trial delay; group mean and standard errors for two groups of six rats (deprived and enriched rats) 63

9 Resistance to change as a function of disrupter and schedule component 73

10 Log proportion of baseline responding maintained under conditions of disruption due to pre-feeding

11 Log proportion of baseline responding maintained under conditions of disruption due to novel objects 
12 Log proportion of baseline responding maintained under conditions of disruption due to extinction

13 Mean proportion responses to $\mathrm{S} 1$ by enriched and deprived rats

14 Box and whisker plot of the matching line slopes, and mean matching lines for enriched, semi-enriched, standard and deprived groups

15 Arithmetic means for enriched and deprived rats response rates as a function of reinforcer density, on a single schedule

16 a) the small continuous access box, b) the large continuous access box and c) the T-maze

17 Mean contact with objects (/30) as a function of consecutive morning or afternoon point samples

18 Mean time samples occuring on the objects side $(/ 30)$ as a function of consecutive morning or afternoon point samples

19 Mean contact preference for the four dimensions of semi-enrichment and the total semi-enriched condition

20 Preference for nine cage options as measured by the continuous access box and T-maze

21 Behaviours shown by rats in the presence of nest boxes and shredded paper

22 Demand data demonstrating straight and curved line fits

23 The demand curves and fitted lines for one subject across three conditions

24 The mean values of $L$ and pmax for the fitted line of 12 demand curves 
of four subjects, for all conditions

25 Intensity (response rate at FR2) and elasticity (last FR with at least one reinforcer earned) of demand for FR2 Group (FR2 steps)

26 Intensity $(L)$ and elasticity (pmax) of demand for FR10 Group (FR10 steps)

27 Intensity $(L)$ and elasiticity (pmax) of demand for six commodities (FR10 steps) 


\section{LIST OF APPENDICES}

APPENDIX

PAGE

1 Individual data from Experiment 1; emergence, open field and

Hebb William's maze

2.1 Individual raw data from Experiment 2.1: correct choices: T-maze and experimental chamber

2.2 Individual response ratio data from Experiment 2.2: response in S1/ total responding

2.3 Individual matching line slopes and biases and ratios of responding to $\mathrm{S} 1 /$ total from Experiment 2.3

2.4 Herrnstein's hyperbola individual $K$ and $R o$ values from Experiment

3.1 Time spent contacting or cohabiting with objects (out of 50) from

Experiment 3.1

3.2 Choice for the non standard option (out of 60) from Experiment 3.2219

3.3 Choice for the non standard option (out of 60) from Experiment 3.3220

4.1 Parameters of individual demand curves 222

4.2 Parameters of individual demand curves $\quad 224$

4.3 Parameters of individual demand curves 226 


\section{Chapter 1:}

\section{The Behavioural Effects of Deprived Environments}

\section{Aims of the Thesis}

This thesis documents an investigation into the behavioural welfare of the rats kept at the Victoria University of Wellington (V.U.W.) animal behaviour laboratory, and by extension those kept under similar conditions throughout New Zealand. There were two inseparable aims of this research. One was to understand as much as possible about the impact of caging environment on the welfare of rats used in laboratories, the other was to evaluate some of the most common procedures used to quantify behaviours that are considered indices relevant to welfare.

The assessment of welfare was broken into three related sections which address the following issues: first, what are the behavioural effects of deprived environments, and are these effects present in the rats kept in this laboratory? Second, are the current methods for measuring the behavioural effects of environmental restriction valid? Finally, what is the best way to provide an improved caging environment for laboratory rats?

The second question is addressed in Chapter 2 which discusses the validity of a common behavioural procedure, the Hebb William's maze. However all four of the chapters of this thesis used different procedures that relate an animals behaviour to its welfare. These procedures are traditional maze and open field methods, operant procedures, preference and economic demand procedures. In each chapter, steps have been taken to inspect the validity 
and reliability of the procedures by comparing them across experiments, to other procedures used in this thesis and to previously published results.

\section{$\underline{\text { Definitions }}$}

There is a lot of disagreement about the definitions of the key terms in animal welfare. This may be due in part to the diversity of people working in the area; ethologists, psychologists, veterinarians and so on. So this chapter shall begin by providing definitions for the key terms used throughout this thesis. The definitions used here are generally drawn from the traditional environmental enrichment literature that makes up the bulk of the studies cited in this section. Otherwise, preference is given to the most concrete and value-free definition of a term, even when this greatly reduces the scope of its meaning. My definition of Animal Welfare itself includes any injury or reduced capacity of the animal regardless of the animals subjective state or feelings.

Readers who disagree with the definitions provided should note that I have erred on the side of caution, in order to avoid misunderstandings, and that the experimental findings are in no way affected by the definitions chosen for their description.

\section{Animal Welfare is compromised}

a) when the animal is significantly impaired in its ability to adapt to the contingencies of reinforcement and punishment operating in its chronic environment.

b) when the contingencies operating in the environment will not support a varied behavioural repertoire without abnormal behaviours, regardless of the animal's efforts to adapt to it. 
Cognitive Behaviour: 'Cognitive' refers to the acquisition of a behaviour (Learning), maintenance behaviour in the presence of positive consequences (Memory), and maintenance of behaviour in the absence of positive consequences (Persistence). The definition used here is drawn from the traditional environmental enrichment research which form the basis of Chapter 1.

Emotional Behaviours: Behaviours (or specific intensities of behaviour) resulting from both inherited tendencies, and the subsequent sensitisation (or habituation) that results from simple exposure to the stimulus. Emotional behaviours range from exploration to fear. Exploration involves approaching and possibly touching a relevant stimulus whereas fear responses involve avoiding a stimulus and might include freezing in place, moving away or burying behaviour.

Enriched Environment: A physical environment with a high degree of complexity when compared with the deprived environment; normally including greater area, a larger group size, and containing various fixtures and objects. It is not used here to describe any quality of the animal, nor any dependant variable in the experiment.

Deprived Environment: A small cage containing a single animal, usually measuring about $20 \mathrm{~cm}$ sq. and containing some bedding material like wood chips. Environments that lack complexity but may not meet these specific dimensions are referred to more generally as restricted.

Restricted Environment: A less specific term referring to environments of the deprived type, that might not meet the specific definition of deprivation that is used here.

Socially Housed: A condition with group sizes larger than the standard conditions, and normally a larger sized cage to encompass them, but no objects or fixtures and so not a full enrichment condition. 
Standard Environment: The normal cage conditions of the laboratory in question, usually the V.U.W. Learning laboratory and as described in Experiment 1.

Standard/Enriched/Deprived Rat: A rat that occupies or has occupied the specified condition. It does not refer to the presence or absence of any measurable effect of this occupation. Traditional Environmental Enrichment Studies: Procedures that involve keeping subjects in enriched and restricted housing conditions and then measuring the differences between them (post. hoc.) using extra-cage procedures. This is contrasted with Modern Environmental Enrichment Studies which began around 1982 (Chamove, 1997) and involved attempting to improve an animals environment, and assessing the effect by measuring the naturalness or complexity of the animals behavioural repertoire (in the cage) before and during the attempted enrichment.

\section{Statements About Laboratory Rat Housing}

Confinement is often the most prominent area of concern when studying the welfare of an animal, especially in a behavioural laboratory where invasive procedures are rarely employed.

"What is increasingly giving cause for public concern is that animals might be suffering, not only in experiments, but because their housing is in some way inadequate." (Birke, 1988 cited in Rose 1994).

On one side of the argument there are those that assume the laboratory housing of rats is unacceptable. This is illustrated by the following exert from an Australian Animal Liberation publication. 
"Regarded merely as experimental 'tools', laboratory animals such as rats suffer constant stress from being handled and kept in close confinement." (Pope, 1991). Despite such claims the findings on handling are fairly clear cut, for example rats have been shown to find handling by humans reinforcing and they will perform tasks to get access to it (Candland et. al., 1962; Davis \& Perusse, 1983). However, there is no such reassuring evidence relating to rats' laboratory housing. Furthermore, concern about rat caging has been raised by less partisan publications (for example, the journal 'Animal Technology') carried the following statement...

"The view put forward here, therefore, is that current housing systems for rats are ethologically, physiologically and psychologically damaging, inappropriate and restrictive and should gradually be replaced." (Batchelor, 1993).

Meanwhile, some researchers seem all too ready to minimise the possible gains to be made by making laboratory housing less constrained. Although there are no general statements against the need for enrichment it is inappropriate in some specific circumstances as it can introduce forms of stimulation that act as confounds to the experimental variables. There are how ever a plethora of objections to any specific form of enrichment which are not justified on any specific basis. Take for example, one of the main ways to reduce confinement - increasing the size of the cage. Chamove (1989) wrote that "It is hard to imagine a case where simply increasing usable cage space would not constitute an enrichment procedure (but some disagree)".

The idea of increasing the size of rats cages seems to have meet with a disproportionate amount of opposition. It has been argued, for example that welfare and enrichment are 
complex phenomena that cannot be so simply solved (Rose, 1990). It has been suggested that factors other than space may be important and at least some behavioural requirements can be met in restricted space (Bantin \& Sanders, 1989; Rose, 1990). It has been argued that the shape of the space or social group may be more important than floor area (Stricklin, 1995), and that increases in space may not always be beneficial (Bantin \& Sanders, 1989), and can even be harmful (Bantin \& Sanders, 1989). It is said that domestic rats are not like wild rats and so therefore (presumably) may not have the same need for space (Bantin \& Sanders, 1989), or on the other hand - rats have evolved in and probably prefer closed in spaces like burrows (Stricklin, 1995). Finally, large cages can be difficult for technicians to work with (Bantin \& Sanders, 1989). These points are quite legitimate but the amount of discussion seems to outweigh the number of actual studies on the behavioural effects of larger cages. In some cases the researchers conclude that it is better to make what improvements we can until we are better able to take into account these complexities (Stricklin, 1995). However, in other cases the conclusion is that we can be complacent as long as the animal appears physically healthy and the animals are not "proven to suffer by our practises" (Bantin \& Sanders, 1989). The main problem with waiting for such proof is that relevant investigations are few and infrequent.

A more moderate position would be that some housing conditions may be significantly compromising of the laboratory rat's welfare, and others are probably not. The most important thing to do is to find a way to tell the difference between these two extreme positions. It may be that little or no change in laboratory caging is necessary, but this cannot be assumed in the absence of evidence. It is our obligation as animal caretakers to doubt the adequacy of the current conditions unless we can prove otherwise, and to try to prove 
otherwise. The specific housing condition investigated by this thesis was that of rats serving as subjects in New Zealand's behavioural laboratories (as determined by the authors anonomous survey, 1995).

First, we needed to know what the potential damaging effects of confinement were and how to measure them. Second we had to determine whether such impairments were present in the animal under investigation and finally we needed to know what cage modifications would be beneficial in ameliorating the cage conditions (if necessary). This section deals with identifying and quantifying the behavioural effects of environmental restriction.

Researchers working with rats have the (largely neglected) advantage of a significant body of research that I shall refer to as traditional environmental enrichment research. The field was initiated by Hebb in the 1940's. Hebb (1947) determined that rats raised as domestic pets performed better in mazes than their laboratory raised counterparts. This led to hundreds of different studies that used standardised tests to compare rats raised in enriched environments with those raised in single subject (deprived) laboratory cages. Enrichment studies provided animals (usually rats) with housing conditions that differed in complexity. Typically, one group was individually housed in small bare cages, another was group housed in large cages that often contained objects and/or fixtures. These studies were most common in the sixties and seventies (Will et. al., 1976; Woods et. al., 1961 for example). The basic form of these experiments is still used, often in conjunction with drugs as a treatment for different forms of brain damage (Rose, 1988). Over the years a number of tests were developed that differentiate between the rats from these enriched and deprived environments. 
The traditional enrichment area was reviewed by Renner and Rosenzweig (1987). They concluded that environment enrichment produces reliable improvement in a wide range of brain parameters as well as marked emotional and cognitive advantages. However, the traditional enrichment researchers rarely discussed their results in terms of their welfare implications. Although Rose (1988) noted that "many of the behavioural changes consequent on enriched conditions... can be seen as contributing to an overall increase in functional efficiency". By appreciating the assumptions of the existing research, the data from enrichment procedures should be able to be applied to questions of welfare.

Traditional enrichment dealt mainly with the theoretical aspects of the effects of environment on brain and behaviour. Thus it tended to see the effects of enrichment as improvements, using the deprived status quo as a baseline. The same procedure could easily be adapted as a test relating to welfare if the enriched conditions was used as the baseline, and any deficits shown by the deprived rats were used as indices of compromised welfare. It is not difficult to equate the deficits shown by deprived rats with compromised welfare and this will be discussed in detail later in this introduction.

The behaviours of deprived rats have been found to differ from those of enriched rats in several key ways (for example, Renner \& Rosenzweig, 1987). These differences were referred to as deficits and were generally divided into two groups. First, there are emotional deficits of which the open field is by far the most frequently used quantification, this is dealt with in Section 1.1. Second, there are the cognitive deficits of which the errors on a HebbWilliam's maze are the pre-eminent example, these are addressed in Section 1.2. Other 
deficits that do not clearly fit in either category are grouped in a final section called Other Behavioural Effects of Environmental Deprivation (Section 1.3).

\section{1) Emotionality}

\section{The Open Field Procedure}

Most of the following review is concerned with the open field, as it is by far the most commonly used measure of emotionality. The open field is an arena enclosed by a wall and divided into sections (see Experiment 1). The number of sections entered within a certain period of time is recorded, often on several consecutive days.

\section{Open Field Results}

Deprived rats are normally described as less active than deprived rats in an open field, but the actual findings are mixed. Some studies support the idea that deprived rats are less active (Ader, 1965; Gill et. al., 1966; Holson, 1986). Others found that enriched rats are less active (Brown, 1968; Denenberg \& Morton, 1962). Finally, some studies have found no significant group differences (Morgan, 1973; Smith, 1972). Presumably, activity is affected by variables that are not controlled across these experiments, and possible candidates will be discussed at the end of this section.

\section{Conclusions Based on Open Field Results}

Some researchers speak only in terms of activity without hypothesising a cause for any differences (Morgan, 1973, for example). However, open field inactivity is normally considered to be a result of fear. The actual words used in the literature include; emotionality (Ader, 1965; Denenberg \& Morton, 1962; Henderson, 1966a), hesitancy (Gill et. al., 1966), or 
freezing (Holson, 1986). The function of emotionality-based inactivity is to make the rats less conspicuous to predators thus it is included here in the category of fear responses. Attempts have been made to correlate this behaviour with variation in heart rate. However different activity levels make this difficult, by introducing a strong confounding variable in the form of activity induced tachycardia (Candland \& Nagy, 1969).

The other side of the coin is that the higher activity levels of enriched rats which have been taken to indicate lower fear levels (Candland \& Nagy, 1969). Thus enriched rats are considered to be less fearful, and more exploratory (Brown, 1968; Smith, 1972).

\section{Open Field Summary}

The lack of an agreed definition for the terms like emotionality and exploration has resulted in some variation in the way they are used. Renner and Rosenzweig (1987) state that the open field may elicit both fearful and exploratory behaviour "but it does not do so in such a way that we can, post hoc, disentangle them". This suggests, perhaps correctly, that emotionality and exploring are rather complex phenomena only loosely related to activity levels. However, these same terms are often used more simply with reference to open field results. Fear normally refers to inactivity and exploration describes activity.

All of these terms used to describe deprived rats behaviours (fearful, etc.) indicate that the less active animals may be suffering from compromised welfare. It is implied that the emotionality is a lasting and generalised quality of the animal rather than something specific to the open field test, and that an emotional animal will spend more time in a state of fear than an unemotional one. There is indirect evidence for this conclusion in that treatments that 
enhance fearfulness (electric shock), generally result in reduced open field activity, and vice versa, whilst neutral treatments have no effect (see Table 1.1). For example, the open field activity of rats is often increased by handling, or treatments that involve handling (Doty \& Doty, 1967; Henderson, 1966; Holson, 1986). Handling may act by reducing the rats overall emotionality and/or by reducing the aversiveness of being transferred by hand from the homecage to the open field (Spence \& Maher, 1962). Such handling may even counteract the effect of environmental deprivation. For example, enriched and deprived rats previously trained on a Lashley stand (with associated handling) did not differ in activity levels, in an experiment where similar unhandled groups showed the expected activity differences (Gill et. al., 1966).

On the other hand, Holson (1986) suggested that enriched rats are more active because of initial attempts to escape from the field due to fear of the experimenter, rather than because of a lack of fearful freezing. Holson interprets escape behaviour as only marginally less emotional than freezing. Renner and Rosenzweig (1987) suggest using video observation so Table 1.1

Interventions that result in changes in open field activity levels

\section{Result}

Increased activity Handling

Previous open field exposure

Hebb-Williams maze

training

Sexual experience

Decreased activity No effect on activity
Electric shock

Handling

Dark rearing

\section{Citation}

Doty \& Doty 1967

Henderson 1966b

Holson 1986

Henderson $1966 b$

Smith 1972

Broadhurst 1956

Henderson 1966 a

Henderson 1966a

Broadhurst 1956

Gibson, Walk \& Tighe 1959 


\section{Visual experience $\quad$ Lavallee 1970}

that the presence of an experimenter does not affect the rats behaviour. Inconsistent results may be attributed to emotional rats tending to freeze or attempt escape thus providing activity scores that are low or high compared with controls (Archer, 1973). This theory may have merit for explaining Holson's data, but there are more likely explanations for the discrepancies in the wider literature.

Confounding Variables of the Open Field: Session Length, Enclosed vs Open Fields and Light Levels

The lack of a standardised open field apparatus or procedure leaves the door open to a great number of confounding variables, in fact it is surprising that there is such a high degree of consensus between researchers as to the meaning of open field data. Walsh and Cummins (1976) wrote that

"almost every physical characteristic of the apparatus, its surroundings and every procedural step have been widely varied".

Activity levels in the open field can change over time and the rats environment experience affect the extent of this change. Over consecutive sessions enriched rats (Holson, 1986; Zimbardo \& Montgomery, 1957) and rats in general (Henderson, 1966a; Smith, 1972) show decreased activity, while shocked rats show increased activity in a second session (Henderson, 1966b). Deprived and handled rats showed no changes in activity (Holson, 1986). Such variations in activity cast doubt on the validity of using a single overall total to describe the behaviour, particularly as the rate of habituation may be affected by previous experience. Activity levels decrease within sessions as well, (Woods et. al., 1960: 
Broadhurst and Eysenk, 1969 cited in Walsh and Cummins 1976). However it is not clear whether these changes also vary with environmental experience.

Some studies have used an enclosed maze instead of an open field. They found that deprived rats were more active (Zimbardo \& Montgomery, 1957) or no different to (Forgays \& Read, 1962) enriched rats in Y-maze activity, and more active in a five choice maze (Sackett, 1967). As rats are 'thigmotaxic' (wall-seeking), a small enclosed environment may seem 'safer' to the deprived rats. Thus a close walled environment may not elicit fear responses from rats. In the absence of a fear response it is possible that the deprived rats would be more active to compensate for the lack of stimulation in their home environment.

Hall et. al. (1997) used variable light levels in conjunction with socially reared and deprived rats. The deprived rats were less active only under the more aversive (brightly lit) open field conditions, and they showed greater avoidance of the brightly lit open field than an open field lit by red light.

\section{Conclusion}

A field that is (for example) very bright or large, might produce a ceiling effect with all of the subjects freezing. A very enclosed or dim maze might not cause any of the subjects to freeze. These floor or ceiling effects would indicate that the researcher did not have the independent variable at the right intensity. The effects of such confounds are limited as they invalidate specific results rather than the procedure in general. If the open field is a valid measure of fear then low scoring animals will show a typical freezing posture, while the high scoring animals will show normal exploratory movements with their bodies and noses. A priority 
should be to identify the independent variables that cause fear in the open field, and to develop a standardised procedure that represents their effective intensity.

Measures Other than Activity in the Open Field: Novel Objects, Defecation and Emergence Sometimes behaviours other than activity are observed using an open field, alternatively behaviours exhibited in other equipment are used as indices of emotionality. These results are generally consistent with the open field activity data.

Some studies move beyond the empty field to assess the effect of novel objects on rats from enriched and deprived rats. Usually the rat is placed in an arena with a number of objects. Exploratory behaviours are categorised and quantitative measures are taken. Enriched rats may show greater levels of investigation of manipulatible objects (Widman \& Rosellini, 1990), but this effect is not usually found (McCall et. al., 1969; Renner \& Rosenzweig, 1986). The more common difference is that enriched rats show more diverse exploratory behaviour (Renner \& Rosenzweig, 1986; Widman \& Rosellini, 1990). Even socially housed (rather than fully enriched) rats contact objects more, and contact a greater variety of objects. They show greater manipulatory behaviour, and more rapid loss of interest in the object and return to previous behaviour patterns (Einon \& Morgan, 1976). Conversely stressed rats showed the same amount of contact as controls, but their exploratory behaviour is less diverse (Rosellini \& Widman, 1989).

Renner and Rosenzweig (1986) write that exploration creates a situation where learning is likely to occur, but also exposes an animal to risk. Rosellini \& Widman (1989) extended this by suggesting that shocked rats were sensitised to predation (more fearful), and they show 
less of the 'diverse' behaviours which would require longer to disengage from an object if a threat arose.

Defecation rates are also thought to reflect fear (Henderson, 1966a; Luchins \& Forgus, 1955). Deprived rats usually defecate more than enriched rats in the open field (Luchins \& Forgus, 1955; Denenberg \& Morton, 1962-elevated maze). Ader (1965) did not find this difference and defecation did not occur frequently enough to be used as a measure by Forgays $\&$ Read (1962). Candland and Nagy (1969) did not find a correlation between heart rate and field defecation despite the fact that both are thought to reflect fear or emotionality.

Generally defecation is thought to be a less sensitive measure of emotionality than activity, however it is consistent with the activity measure in that it is affected by the same variables. For example, handling decreased defecation (Doty \& Doty, 1967-young or old rats; Henderson, 1966b cf. Broadhurst, 1956) as did previous experience of the test (Broadhurst, 1956) and sexual experience (Broadhurst, 1956), while shocked rats defecated more (Henderson, 1966a) and dark rearing (Gibson et. al., 1959), visual experience (Lavallee, 1970) and trauma (Bingham \& Griffiths, 1959) had no effect. Defecation levels vary between sessions in a similar way to activity levels (Henderson, 1966a; Henderson, 1966a; Doty \& Doty, 1967).

Broadhurst (1956) favours the defecation frequency over activity levels as a measure of emotionality because it is less sensitive thus reducing the possibility of a 'false positive' finding. However other researchers favour activity as the more sensitive measure (Doty \& 
Doty, 1967), and in at least one case (Denenberg \& Morton, 1962) enrichment had a significant effect on defecation when activity did not.

Emotionality is also measured as latency to emergence into an open or unfamiliar place. This can be tested in a specially designed box or runway, or the start box of a maze (see Experiment 1 for an illustration of the emergence box). Longer latencies are thought to indicate higher levels of fear. If enriched rats were less fearful they would be expected to emerge sooner and/or more frequently than deprived rats (Renner \& Rosenzweig, 1987).

Holson (1986) found that enriched, and to a lesser extent, handled rats made more turns into the light side of an 'emergence box'. These findings indicate that enriched rats show less avoidance of novel environments. Enriched rats were also quicker to start running in an elevated maze (Luchins \& Forgus, 1955) and had short latencies in a Lashley jumping stand (Gill et. al., 1966).

\section{The Relation Between Fear and Novelty}

The open field, emergence and novel object results are consistent with the deprived rats showing greater avoidance of novelty (neophobia; e.g. King, 1970). Rats from deprived environments may take longer to make contact with novel objects or areas, and their subsequent contact may be limited in amount and variety. When the behaviour is described as caution or simply avoidance of novelty, it is less clear whether this behaviour indicates poorer welfare. 
Blanchard et. al. (1974) suggested that novelty reliably produces fear in rats and that novelty induced fear is the key element of emergence and activity behaviour. In an experiment where some subjects were pre-exposed to the equipment to reduce it's novelty, they found increased activity and reduced emergence latency. They also showed that rats found novel environments aversive, as they would cross an electric grid to return to their home cages. Thus it appears that rats are biologically prepared to fear novelty.

Enriched animals have often been exposed to novel stimuli that do not have any harmful potential, thus their natural fear of novelty may have been diminished by this experience. In contrast, deprived animals have not experienced novel stimuli and so are equally sensitive to the possibilities for reinforcement or punishment. Thus the enriched rats advantage may be largely due to the open field being more similar to the enriched home cage than the deprived home cage. Thus the open field will be less novel to the enriched rats in terms of space, light levels, openness or some combination of these and other variables. This 'transfer effect' between the enriched environment and the open field, does not deny that the deprived rats experienced greater fear than the enriched rats. It does not deny that this tendency to be more fearful occurs in a wide variety of situations, but it gives a simpler explanation of the phenomenon with less use of problematical intervening variables like 'emotionality'.

\section{The Relation Between Neophobia and Welfare}

Good animal welfare can be defined as the absence of any evidence of physical or emotional suffering, including "fear and distress" (F.A.W.C., 1992). The deprived rats tendency to avoid novelty suggests that they experience more distress when a change is made in their 
environment (such as experimental interventions). Thus they are arguably experiencing more compromised welfare.

Another definition of welfare is that the animal should show a similar repertoire of behaviour to a wild animal. This has a number of difficulties in that the laboratory environment will never, even roughly, resemble a natural setting, and it is unclear how different the captives rat's behaviour may be from that of the wild rats before welfare is considered to be compramised.

The comparison of wild and domestic animal does, however, have implication regardling welfare. Chamove (1994), for example, wrote that wild animals frequently encounter and even seem to seek out fear inducing situations. This might suggest that stress levels should be optimised rather than minimised, in which case results from the open field are more ambiguous. The optimal level of stress would be determined by a great many factors, and is almost impossible to determine. Theoretically the optimal level of exploration should maximise the animals chances of encountering a reinforcing stimulus, but minimise the risk of encountering a punishing stimulus, with the appropriate level of exploration being proportional to the predominance and intensity of these contingencies.

Roeder et. al. (1980) exposed enriched and deprived rats to polecat predation in a large environment. Initially the enriched rats were more heavily predated, but after a while the enriched group became superior to the deprived rats at avoiding the predator. So neophobia may be (at least initially) an advantage in natural situations where there are predators, poisons 
and so on. It is harder to interpret the latter part of the experiment as the surviving animals were not a random sample of those that began the experiment.

\section{In the Laboratory Setting}

The laboratory rat is not living in the wild, and so the concept of "natural behavioural repertoires' may not be particularly helpful. In a behavioural laboratory life threatening, or even painful, environments are not typically present. Even when the laboratory does provide an aversive situation it is normally inescapable and /or made worse by fear responses. In the lab, being neophobic is not adaptive. Neophobic animals simply experience unnecessary stress in response to unthreatening stimuli, or respond more intensely to inescapable aversive stimuli. Also, animals that are difficult to handle due to escape or aggressive behaviours are unlikely to be retained or bred from. As it is the laboratory environment that is being discussed here, it seems likely minimal neophobia in a rat is the most adaptive trait, and best for the animals welfare. Thus conditions that reduce neophobia in rats are best for their welfare. In terms of practical considerations an animal with reduced avoidance of novelty would also be easier to handle and manipulate.

\section{Conclusion}

The open field is a common and widely accepted procedure for measuring the degrees to which subjects avoid novelty. The procedure could be further standardised to increase reliability. The best procedure would probably include a large field and short, repeated sessions observed by a remote method such as a video camera. Other factors such as lighting, field colour, materials and dimensions should be described in enough detail to allow replication. The emergence box, though less commonly used, could provide a second 
indicator of this neophobia. However, the relevance of both of these tests would depend on the individual's definition of welfare.

\section{2) Cognitive Behaviours}

\section{The Hebb William's Maze Procedure}

The Hebb Williams maze was originally described as an animal intelligence test, and is now called a test of problem solving ability or of learning (Renner \& Rosenzweig, 1987). It is a square arena with start and goal box in diagonal corners, and movable barriers (see Experiment 1). Subjects are given experience with the arena and position of the food, and then individually trained until they can run from start to goal box, around a pattern of barriers, nine times within sixty seconds. Next, rats are tested with twelve different barrier patterns and entry into areas that do not lead to the goal box are scored as errors.

\section{Hebb William's Maze Results}

Rats from enriched environments make significantly fewer errors on the Hebb-Williams maze problems (Denenberg et. al., 1968; Forgays \& Forgays, 1952; Forgays \& Read, 1962;

Hymovitch, 1952; Smith, 1972; Woods, 1959; Woods et. al., 1961), at least when the maze is stationary (Brown, 1968). These differences in performance may be maintained 75 (Hymovitch, 1952) and 330 (Denenberg et. al., 1968) days after termination of the enrichment condition. When the control group was socially housed, enriched rats did not show superior performance (Venable et. al., 1988). So social housing may be an important aspect of enrichment, at least as it is measured by maze running. 


\section{Interpretations of the Maze Results}

Enrichment ameliorates the impact of deficits in maze running caused by brain lesions. For example Hughes (1965) found that pre-operative enrichment reduced the impact of hippocampal lesions on maze efficiency. Post-operative enrichment also reduces the maze deficits of rats subjected to a range of injuries including; cortical lesions (Schwartz, 1964; Will et. al., 1976; Will et. al., 1977; Pacteau et. al., 1989; Rose et. al., 1992), and Xirradiation (Shibagaki et. al., 1981). Kolb and Elliot (1987) found significant differences in maze performance only in the lesioned enriched and deprived group, and not in the normal control group. However, the improvement produced by enrichment rarely demonstrated an interaction between the deficit (e.g. lesion) and the enrichment, normally enrichment has a roughly equal effect on lesioned and control animals. Thus enrichment does not reverse the effects of lesions, but provides an independent advantage.

The Hebb-Williams maze finding is generally supported by experiments that used other types of mazes. Enriched rats superior performance was replicated by Holson (1986) with a maze of his own design, as well as with the Warner-Warden and inclined plane mazes (Bingham \& Griffiths 1952). Hypothyroid enriched rats make fewer errors than hypothyroid deprived rats on a symmetrical maze (Davenport et. al., 1976).

In addition Luchins and Forgus (1955) reported that enriched rats maze performance was more flexible and they used more variable paths to the goal box of a specially designed maze. They were quicker to learn to take a long route when guided and are quicker to revert to the short route afterwards. Therefore, a researcher arguing against the validity of the HebbWilliam's maze is, by extension, maligning a wide range of open maze procedures. 


\section{Conclusion}

There are several possible explanations for the better performance observed with enriched animals on mazes. The maze task was designed to test animal intelligence or problemsolving and better performance was expected to be the result of a cognitive advantage. Rats from enriched environments show superior maze performance in the absence of measurable differences in exploration (Brown, 1968; Forgays \& Read, 1962). Thus the improved performance is normally considered to be a global cognitive advantage and it has been linked to brain changes discovered in other enrichment studies (Denenberg et. al., 1968).

The Results are Affected by Non-Cognitive Variables: Simple Transfer, Perception and Exploration

An alternative explanation is that enriched environments are more like the maze than simple cages, and enriched rats are simply more practised at moving around barriers. This may explain why large environments with barriers produce rats that make fewer maze errors (Brown, 1968; Hymovitch, 1952). However, this hypothesis would not explain the superior performance of rats from enrichment conditions that do not provide any barriers (Denenberg et. al., 1968, for example).

Theories that claim that one specific part of the enrichment (barriers, space etc.) is responsible for the maze findings are unconvincing because of the wide range of enriched conditions used between laboratories normally reveals at least one that did not include this dimension. 
Treatments other than enrichment also affect maze performance. For example, rats exposed to complex visual images make fewer errors on the Hebb William's maze (Lavallee, 1970). Visual experience does not affect open field performance (Gibson et. al., 1959; Lavallee, 1970), which suggests that the open field and the maze are measuring independent qualities in the animal. However, it is more significant that this finding suggests that the Hebb William's maze measures a perceptual ability rather than a cognitive one (under the definition of cognition used here). This finding was replicated by Walk (1958) who found that the rats performance was enhanced even further by visual and tactile forms of experience (using an elevated maze).

Many studies have suggested that the enriched rats advantage is more perceptual than intelligence based. Hymovitch (1952) suggested that the enriched environment allows for greater visual and non-visual perceptual learning. This improved perceptual ability then allows enriched rats to make greater use of extra maze cues. This explanation is consistent with the enriched rats poor performance when the maze was rotated between problems (Brown, 1968; Hymovitch, 1952). If this explanation is valid then the size of the enriched environment should be crucial because it would allow rats more opportunity to learn to use distal cues (Brown, 1968).

Deprived rats performance also equalled that of enriched rats when food deprivation was extreme (Woods et. al., 1961), or foot-shock was applied in the maze (Woods et. al.). This may suggest that the deprived rats poor performance in mazes may not be due to an absolute inability to do the task accurately, but because the reinforcement offered is in some way less 
effective with them. This could be for a number of reasons. Crossing the maze might be more aversive for deprived rats, the food may be less reinforcing or there may be other sources of reinforcement (like exploring the novel environment) that compete with the food.

Alternatively deprived rats may make more errors than enriched rats because they show more exploratory behaviour in the maze, because the maze is relatively more novel compared with a deprived environment, than compared with an enriched environment (Zimbardo \& Montgomery, 1957). The previous section showed that rats from deprived environments normally show less exploratory behaviour than rats from enriched environments, but suggested that the opposite may be true in enclosed spaces such as provided by the maze barriers. It is also possible that in an environment as familiar as the Hebb William's maze after preliminary training, the enriched rats' activity has habituated to low levels, while the deprived animals activity has increased. When the rats are discouraged from exploring by extreme food deprivation or foot shock, the deprived rats performance does improve to levels shown by enriched rats (Woods et. al., 1961).

Fearfulness that depends on the novelty of the environment might be neutralised by the habituation and maze training provided to all rats before testing in the maze (Denenberg et. al., 1968). However the deprived rats may still be more reactive to novel barriers even if they are habituated to the arena (Holson, 1986), and a set period of habituation might have an uneven effect on the enriched and deprived rats. Holson (1986) also suggested that deprived rats may avoid the goal box to some extent because they avoid eating in an unfamiliar environment. 
Although an animals emotionality may legitimately contribute to its ability to solve problems emotionality is not normally considered to be a cognitive variable per. se. The maze represents the kind of problem that might be common in a rats natural environment, the ability to navigate around physical features to get to reinforcement. This is an ability that would be affected by both the cognitive skills (perception, insight, memory) as well as the degree of avoidance shown to a novel change in a basically familiar environment (neophobia). However neophobia is not the kind of variable that the original Hebb William's intelligence test was designed to detect nor is it part of most current researchers understanding of what the maze measures.

\section{Conclusion}

In the long run Hebb William's maze findings should probably be replicated by a different task which is not confounded by neophobia and measures a specific ability that is impaired by environmental deprivation. If such a replication occurred, it would suggest that the Hebb William's maze is robust against its possible confounding variables.

\section{The Relation Between Cognitive Deficit and Welfare}

From a welfare perspective an environment that has caused a cognitive deficit could easily be described as detrimental to welfare. It would demonstrate that there is a lack of necessary stimulation in the environment, and indicates that the animal has more difficulty in day-to-day learning than its enriched counterparts. In practical terms such a deficit may make an animal difficult to train or limit the range and extent of its behavioural capacity. It is a variable that would impact on most of the data produced by animal behavioural laboratories. 
Cognitive Measures Other Than Mazes: Visual Discrimination and Performance Flexibility Several studies have studied the effect of enrichment on visual discrimination performance. Discrimination performance is normally quantified as the number of errors made, or time taken, before the subject reaches a certain level of accuracy. The correct choice is rewarded with food or water, the choice is quantified using equipment such as a T-maze, water T-maze or Lashley jumping stand. General enrichment did not affect the ability of rats to learn a visual discrimination (Bingham \& Griffiths, 1952; Gill et. al., 1966; Woods et. al., 1960). However, Krech et. al. (1962) found that deprived rats performed poorly on discrimination reversals. Neither of these findings is consistently reported as some studies find enriched rats do learn a visual discrimination quicker (e.g. Lavallee, 1970) or that deprived rats show no deficit on a discrimination reversal (e.g. Gill et. al., 1966).

Some of the variability of these results may reflect the choice of visual stimuli and their relation to stimuli in the enriched environment. For example, if the rats housing contains the same visual patterns later used as visual discrimination stimuli, they show better performance (Gibson \& Walk, 1956; Walk et. al., 1958; Lavallee, 1970) and even better performance if one form is later used on the discrimination and one is not (Forgus, 1958a). If the environmental forms are only similar to the discrimination stimuli learning is improved even further (Forgus 1958; Gibson et. al., 1959), but not if they are different (Gibson et. al., 1959).

In contrast to these studies, Gibson et. al. (1959) failed to produce better performance in rats even after providing rats with exposure to specific discrimination patterns. This failure may have been due to the stimuli being painted and flat rather than cut-out. Meier \& McGee 
(1959) found that only rats with tactile contact with the visual forms were superior to controls.

These data show that only very specific forms of pre-exposure reliably improved rats ability to learn visual discriminations, and that general enrichment of the environment normally has no effect on initial learning. However deprived rats may be slower to learn a discrimination reversal. This deficit could not be due to differences in exploratory behaviour. Activity differences cannot explain learning deficits that occur with discrimination reversal only, as this would have an equal effect on initial learning (Krech et. al., 1962).

The possibility that deprived rats are impaired in discrimination reversal is interesting in that other findings also point to their behavioural inflexibility. For example Luchins and Forgus (1955) found that after learning to run a maze, deprived rats took longer to learn to take a long maze route when guided and take longer to revert to using a short maze path when it became available again. Davenport et. al. (1976) found that hypothyroid deprived rats bar pressing behaviour takes longer to extinguish under extinction than hypothyroid enriched rats. Morgan (1973) found that deprived rats showed no deficit when learning to move an object or go through a door to get food, but took longer to move an object in a different way, or climb a ladder to a door leading to food.

Less work has been done in the area of learning deficits other than with mazes or measures of activity, so there is no common standardised test. Learning and modification of learning are cognitive abilities which deprived environments could be shown to affect, but few of these findings are well replicated. A valid test of these abilities would have to incorporate a 
minimum of open space, novelty and handling so that it is not confounded by issues of emotionality. Acquisition of a visual discrimination and subsequent reversal could provide a basis for the task.

A reliable finding of deficits in reversal or modification of learning would suggest that deprived animals adapt poorly to changes in reinforcement contingencies. Ideally the task should allow for further investigation of the exact nature of this deficit.

\section{3) Other Behavioural Effects of Environmental Deprivation}

\section{Operant Response Rate, Dexterity and Forgetting}

There are findings that indicate that deprived rats show higher response rates in operant experiments of several types. Ough et. al. (1972) found that deprived rats performed poorly on schedules that differentially reinforce low rates of responding. Coburn and Tarte (1976) found deprived rats contra-freeload to a greater extent than enriched rats, and they responded more during extinction (Davenport et. al., 1976). Deprived rats also pressed a lever sooner and more often than enriched rats when responding was reinforced by light and food (Rose, 1988). Kolb and Elliot (1987) found a whole raft of effects of enrichment on the physical dexterity of normal and frontally decordicated rats. These included superior performance in tongue extension, grooming, beam walking, fore-paw pellet manipulation and sticky tape removal. Finally, time in an enriched environment after initial training, reduced the incidence of successful active avoidance (Parsons \& Spear, 1972). This is one effect that might give enriched rats a disadvantage in learning tasks. 


\section{4) Conclusion}

Traditional environmental enrichment studies have shown the behavioural effects of caging, and demonstrated methods for quantifying them. The deprived and enriched conditions provide well understood behavioural land-marks for determining the levels of behavioural deficit that are unacceptable in terms of welfare.

Although a lot of research has been published in the area of environmental enrichment, few of the findings have been directly applied to the welfare of the animals concerned. Normally the enriched and deprived conditions are constructed to provide the greatest possible contrast, and so increase the chance of producing a significant enrichment effect. However, it is not normally stated whether any of the experimental conditions are equivalent to standard laboratory housing. If they were then the results of the enrichment experiment might provide information about the welfare of animals kept under these standard conditions.

Enrichment studies normally employ the deprived (or standard when included) conditions as a control group. To study animal welfare with these techniques the enriched group would be the control (as the more normal animal), allowing the experimenter to detect behavioural deficits on the part of animal in standard (or other) housing conditions. For example, a rat in a standard laboratory cage could be compared to rats in enriched conditions similar to those in studies of environmental enrichment.

As the aim of environmental enrichment is apparently the removal of a behavioural deficit produced by a deprived environment, a more appropriate term to apply to the techniques when used in the field of welfare might be environment mitigation. In the long term the 
mitigating effects of different aspects of the enriched environment (i.e. space, social contact, manipulatible objects) could be assessed using these techniques. This would provide indications of how best to improve environments.

The open field and related emotionality measures suggest that rats from deprived environments show a greater avoidance of novelty and so would presumably experience greater fear when confronted with unavoidable novelty. Such avoidance is a welfare concern if we regard our goal as minimising stress rather than providing optimal or natural stress level.

Likewise the open field test is useful if we are trying to adapt the animal to a risk-free environment where the loss of learning opportunities is not counter-balanced by better avoidance of predators or other harmful stimuli. A behavioural laboratory would be an example of such a minimally dangerous environment.

The Hebb William's maze is normally described as detecting deficits in problem-solving ability. These deficits in problem solving would seem to indicate that the animals environment had caused it to suffer a cognitive deficit, as much an indication of poor welfare as a physical deficit. It may also produce an animal that performs poorly in everyday learning situations, thus reducing the effectiveness with which it exploits its environment. However it must be kept in mind that maze scores may also be affected by differences in reaction to novelty (as discussed in relation to the open field), or more basic perceptual or motivational characteristics. Despite this the Hebb William's maze is the most well recognised and replicated test of cognitive abilities that is currently available. 
A less ambiguous cognitive test could be based on other learning tasks as discussed in Chapter 2. There is an indication that deprived rats adapt poorly to changes in their environment on a cognitive basis as well as showing avoidance of these situations, but this finding needs further investigation and replication. An informative test could be based on initial and reversal visual discrimination learning. If this was carried out in a response chamber, space and novelty could be minimised and opportunities for habituation to the environment maximised.

The maze and open field tests do seem to have some relevance to animal welfare, although it is not clear cut. A way to approach the assessment of welfare would be to look for the effects of the environment on the animal, in a standardised test environment with well defined demands on their behavioural abilities. Thus we are not studying the interaction between the animal and it's environment, but the state of the animal itself and it's behavioural abilities and reactions. With this in mind tests of more specific behaviours could be constructed, ones that would be more easy to interpret in relation to animal welfare. 


\section{EXPERIMENT 1:}

\section{Evaluating the Standard Caging Conditions}

This experiment provided evidence relevant to assessing the welfare of rats kept under standard laboratory conditions in New Zealand laboratories. The aim was to determine whether laboratory rats kept under standard conditions demonstrated the same behavioural abnormalities that have been shown to result from deprived housing conditions. If behavioural deficits were evident, then a programme would be initiated to determine how the rat housing should be modified.

There are two advantages of doing a priori tests of the baseline welfare of an animal. Firstly, it encourages an open mind about the quality of the animals life under existing conditions, empirical data can challenge those who assume the best or the worst about the animals condition. While it is true of welfare indicators that "absence of evidence is not evidence of absence" (Morton, pers. comm.), it is also true that some animals probably live in captive conditions that do not significantly compromise their welfare. Secondly, the baseline measures may serve as an indicator of the nature and extent of any problem, thus indicating the kind of intervention that may be required.

Most research on enriching the home environments of laboratory rats has relied on preference measures (Denny, 1975; Anzaldo et. al., 1994; Bradshaw \& Poling, 1991 and others) and there is potential for economic demand procedures to extend this approach. However, these studies become difficult to interpret when the subjects do not prefer the alternative environment. Such a lack of preference could mean either that the current environment is as 
good as it can be, or alternatively that significant improvements may be possible but the current options do not produce them. Given this ambiguity, preference and demand procedures should be used in conjunction with other procedures which give a broader picture of the animal's welfare.

In order to decide whether an animal's welfare is compromised, there must be a standard against which the animal's behaviour can be compared. The most common behavioural gold standard is behaviour closely related to the behaviour of a wild animal, (the "natural-living concept" Fraser et. al., 1997). The use of such behaviour to assess laboratory rats is questionable. Firstly, there are no published quantitative ethograms and time budgets for wild Norway rats (the subjects of interest here). Secondly, while laboratory rats can show suprisingly feral behaviour in some situations (Boice, 1977), there are also significant behavioural differences between inbred and wild type Norway rats under other circumstances (Cowan, 1977; Huck \& Price, 1975). Generally, domestic rats are less fearful but otherwise negligibly different from their wild counter-parts. Finally it is not clear whether it is possible or advisable to encourage an animal to behave naturally in its home environment but unnaturally in course of its use by humans. That is, it is unlikely that an animal could behave like a wild rat in its home environment but also tolerate handling, injections and novel test environments without becoming extremely fearful.

Without an acceptable guideline of what behaviours rats should show, or at what levels, existing behavioural data are difficult to interpret. For example, rats provided with nest boxes explore more than rats from barren cages, but they walk and rear less than these controls (Townsend, 1997). Is there any valid reason to believe that the behaviour pattern 
shown by the rats with nest boxes is better than the behaviour of the controls? All that the data demonstrates is that they behave differently.

The enriched and deprived conditions that have been used by the traditional environmental enrichment studies offer an alternative to the use of a wild baseline. The behaviour of animals in extreme laboratory environments may serve as anchor points on a welfare continuum.

The traditional enriched environment was normally the most expansive and stimulating laboratory environment that the laboratory in question could provide and maintain for any length of time. Rats from such an enriched environment could be used as a gold standard instead of wild animals. The construction of the enriched environment has differed between laboratories, but its general characteristics have remained very similar, as have the behavioural benefits produced in subjects compared with rats under deprived conditions.

This chapter has already outlined the extensive evidence of behavioural differences between rats from deprived versus enriched conditions. Ideally the effect of the environmental conditions should be assessed using a battery of tests that sample aspects of the subjects physical health as well as their behaviour. However, behavioural tests have many advantages as a place to begin. They are non-invasive and raise very few welfare issues of their own. Behaviour measures are also very relevant to those subjects that are kept as subjects in a behavioural laboratory. That is, the current experiment could demonstrate how caging environment can act as a confounding variable in behavioural experiments. 
The current experiment examined whether the open field, emergence box and Hebb-Williams maze would differentiate between rats kept under standard New Zealand university behavioural laboratory conditions (as determined by an anonymous 1995 survey performed by the author) and those from deprived, semi-enriched and enriched conditions. The standard conditions were two rats in a $20 \times 23 \times 45 \mathrm{~cm}$ cage with wood shavings as bedding.

\section{Method}

\section{$\underline{\text { Subjects }}$}

The subjects were 35 female Hooded Norway rats from the Victoria University of Wellington breeding colony ( 8 enriched, 8 semi-enriched, 12 standard and 7 deprived). All subjects were $30(+/-2)$ days old when they were differentially housed and $60(+/-2)$ days old at the beginning of the experiment.

\section{Apparatus}

Enriched housing consisted of a $70 \times 40 \times 50 \mathrm{~cm}\left(14,000 \mathrm{~cm}^{3}\right)$ glass display case with a perforated metal lid. The cage contained two large nesting boxes, branches, a cardboard box, a running wheel, several plastic containers, straw and tissue paper. The position of these objects and of the food pellets were changed every ten days. Four manipulatible objects were placed in the cage and changed every day from a pool of 120 objects. Four adult females occupied the cage in addition to the eight enriched subjects.

Semi-enriched housing consisted of two large cages constructed from two $20 \times 23 \times 45 \mathrm{~cm}$ cages with a plastic bottom half and a wire top half joined along the long side. The left half 
of the cage contained four fixed wooden pillars that measured $10 \times 10 \mathrm{~cm}$ at the base and ranged from 5 to $15 \mathrm{~cm}$ in height. The right half of the cage contained four manipulatible objects which were changed daily from a pool of 120 objects. Each cage housed four subjects.

The standard conditions were based on median values from this experimenters (1995) survey of 20 New Zealand animal behaviour laboratories, regarding the size, composition and contents of experimental rats cages. The four standard cages were $20 \times 23 \times 45 \mathrm{~cm}$ in size with a plastic bottom half and a wire top half, and housed two subjects with wood-shavings.

The deprived housing consisted of seven $23 \times 23 \times 26 \mathrm{~cm}$ solid metal cages with wire mesh lids. All of the cages contained wood-shavings and a single subject. All subjects had free access to water and were housed in a colony room with a 12 hour light/dark cycle and maintained at 21C.

An open field measuring $100 \times 100 \times 40 \mathrm{~cm}$ was constructed out of wood and the inside surfaces painted mid grey. The floor was divided into $20 \mathrm{~cm}$ squares with black painted lines. A standard Hebb-Williams maze was constructed according to the instructions provided by Robinovich and Rosvold (1951). The Hebb-Williams maze is a large square maze with entry and exit points on opposite corners and a number of movable barriers that can be used to construct the 12 standardised problems. The open field, Hebb-William's maze and emergence box are shown in Figure 1.1.

Sessions in the field and maze were recorded by a JVC RM-V20U compact video camera connected a JVC HR-D910EA video cassette recorder. 
The Emergence Box

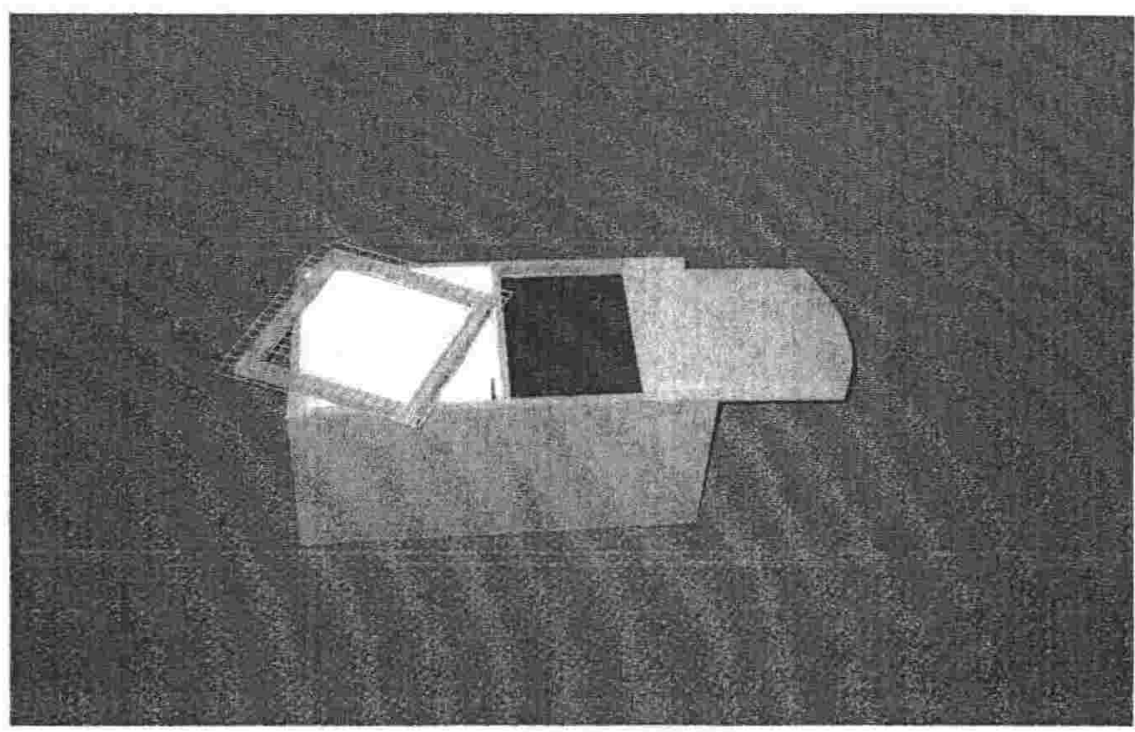

The Open Field
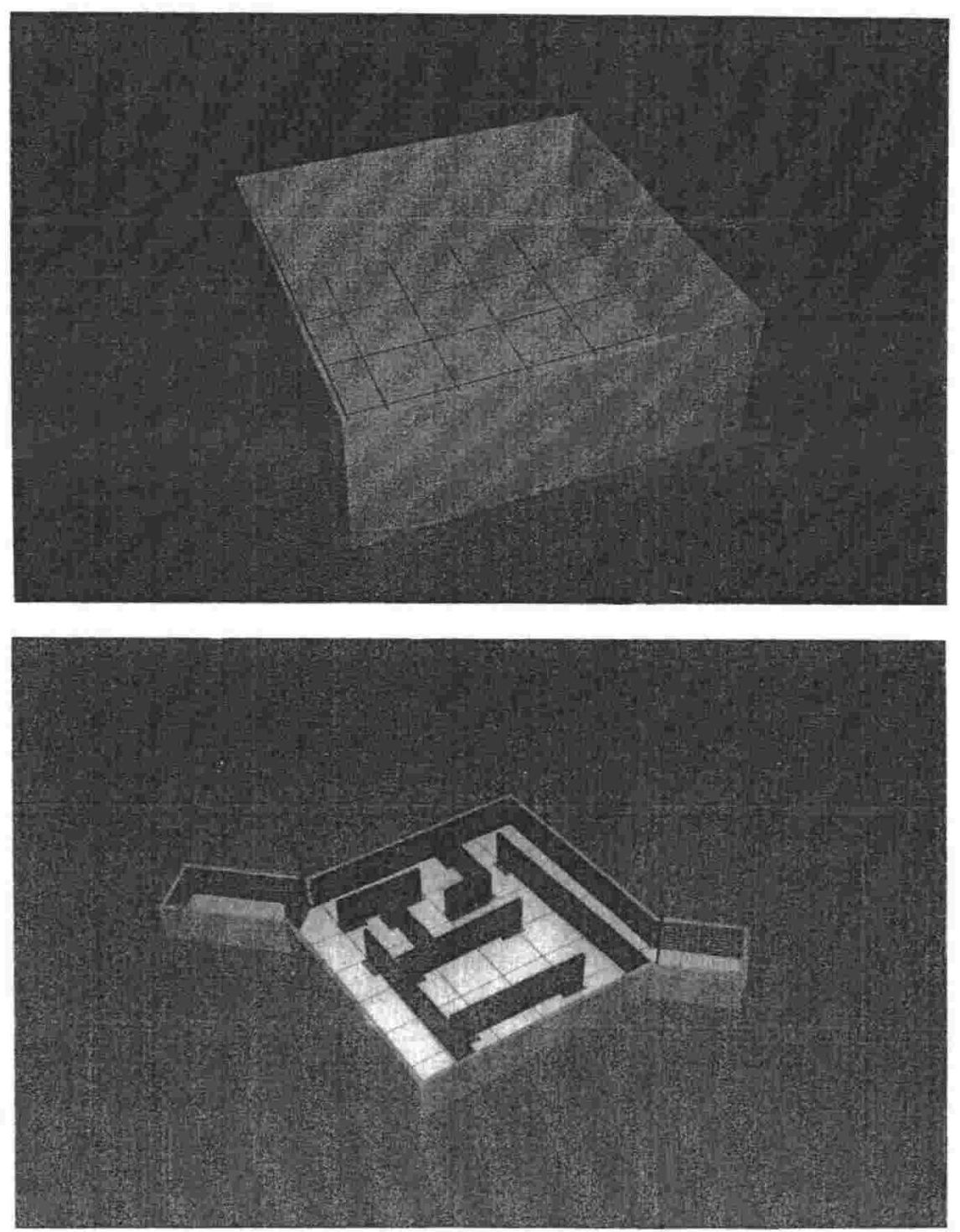

Figure 1.1 Photographs of the emergence box, open field and Hebb William's maze. 


\section{$\underline{\text { Procedure }}$}

The subjects were run in three groups of 12 animals, at separate times. The first two groups were divided into three sets of four with litters split equally between the deprived, standard and semi-enriched groups. At a later date a third group was divided into a set of eight enriched rats and a control group of four standard rats.

All of the subjects remained in their respective housing conditions with minimal handling and free access to water for thirty days. Food was available $a d$. lib. until day 26 when it was restricted to 1 hour of ad. lib. feeding per day (3-4p.m.). This feeding schedule is commonly used in traditional enrichment studies with rats that have not reached their full adult weight (Denenberg et. al., 1968 for example). One hour feeding maintained the rats in a similar condition to adults that are at $80 \%$ of free-feeding weight.

On days 31 to 33 each rat was tested in the emergence box and open field. The rats were carried in their group cages to a darkened room adjoining the testing room, and then individually carried by hand into the testing room. They were placed in the dark side of the emergence box. After ten seconds the door to the lit side of the box was opened and the stopwatch started. Once the rat had all four feet through the door, or after fifteen minutes, the stopwatch was stopped. The subject was immediately placed in the corner of the open field, facing the wall. The experimenter left the room and the field was video-taped from above for ten minutes. Testing occurred between 2-4 pm during the rats active period (give the reversed lighting in the housing room). The testing room was similar to the housing room in dimensions, materials and lighting. Light levels in the open field and lit side of the emergence box were $71.6 \mathrm{~cd} / \mathrm{m}^{2}$. They were fed immediately after testing for the day had 
finished. The open field data were recorded in successive one minute bins throughout the ten minute sessions. Each time a rat's nose entered a new square the score for that interval was increased by one.

On day 34 training on the Hebb William's maze task commenced, followed on successive days by testing on twelve standardised maze problems. This task requires rats to navigate a series of barriers in order to reach a goal box containing food. The maze testing followed the protocols of Robinovich and Rosvold (1951) and a chocolate chip placed in the goal box was used as bait. Each day a new problem was constructed by altering the barrier configuration. The rats ran eight trials, form entry box to goal box. Entry into pre-determined 'error zones' were added to their total error scores. A video record (from above) was kept for precise scoring.

Each Hebb William's maze error zone was given an identifying number. The first time a rat made this error it was scored as an initial error, any repetition of this error in the same, or subsequent trials was scored as a repeated error. Total errors for each rat were the sum of initial and repeated errors. The only difference in procedure between the first two groups was the order in which the sets were tested each day. In Group One the enriched set were tested first and the deprived set last. For Group Two the order was reversed.

\section{Results}

The individual data used in the following results section are shown in Appendix 1. 


\section{Open Field and Emergence}

Figure 1.2 shows the mean emergence latencies for each group. The deprived group showed longer latencies to emerge than any other group, but this difference was statistically significant for the first session only (Kruskal-Wallis ANOVA $\mathrm{H}=16.93, \mathrm{df}=3,35, \mathrm{p}<0.05$ ) and a post-hoc Mann-Whitney $\mathrm{U}(\mathrm{Z}=-3.24,-3.12,-2.92, \mathrm{p}<0.05)$. However, there was a very large standard error for this group. Some of the deprived rats showed very long latencies (up to the 15 minute ceiling) while some showed latencies similar to the other groups.

Figure 1.3 shows the group means for total open field activity during the first two minutes of the first session. This figure shows that there was a tendency, in the first two minutes, for rats from the enriched and semi-enriched groups to enter more squares than rats from other groups (Kruskal-Wallis ANOVA $\mathrm{H}=11.94, \mathrm{df}=3,35, \mathrm{p}<0.05$ ). However when the activity scores were averaged over the whole ten minute session there were no significant group differences (Kruskal-Wallis ANOVA $\mathrm{H}=7.20, \mathrm{df}=3,34, \mathrm{p}>0.05$ ).

The loss of the group differences seen in the first two minutes, when the sample is increased to ten minutes, is consistent with a trend in the data which is displayed in Figure 1.4. Figure 1.4 shows activity levels for each successive minute during the first 10 minute session. The enriched group showed a rapid decrease in activity levels during the session. The semienriched rats also show some decrease in responding, but the standard and deprived rats tended to maintain their activity levels throughout the session. When this drop in activity is expressed as the difference between squares entered during the first minute of the session, and squares entered during the last minute of the session, there is a significant effect of housing condition across all groups (Kruskal-Wallis ANOVA $\mathrm{H}=12.02$, $\mathrm{df}=3,34, \mathrm{p}<0.05$ ). 


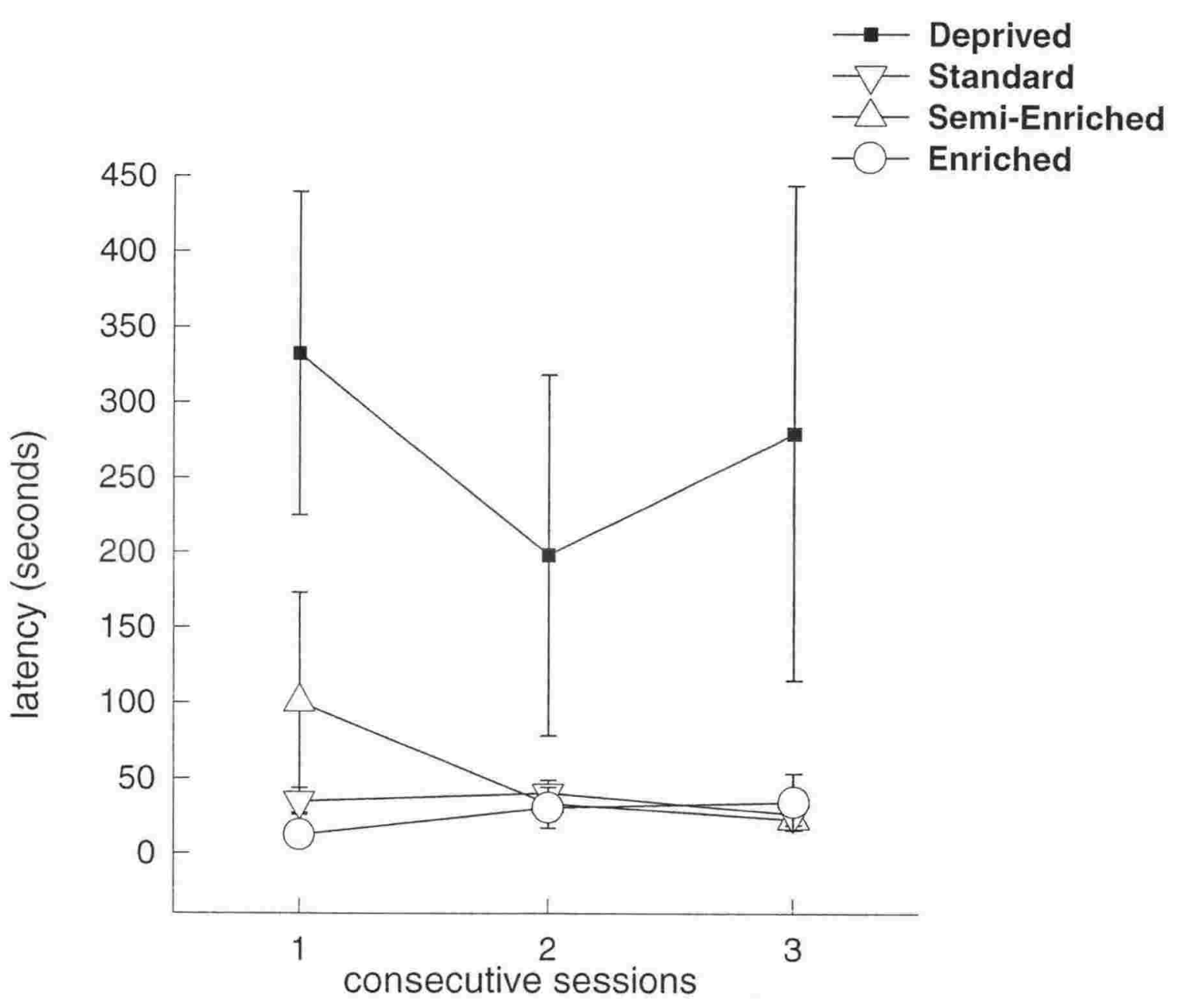

Figure 1.2 Latencies to emerge from the dark half of a box into the light half. Group averages for deprived, standard, semi-enriched and enriched rats, with bars showing standard errors of the mean. 


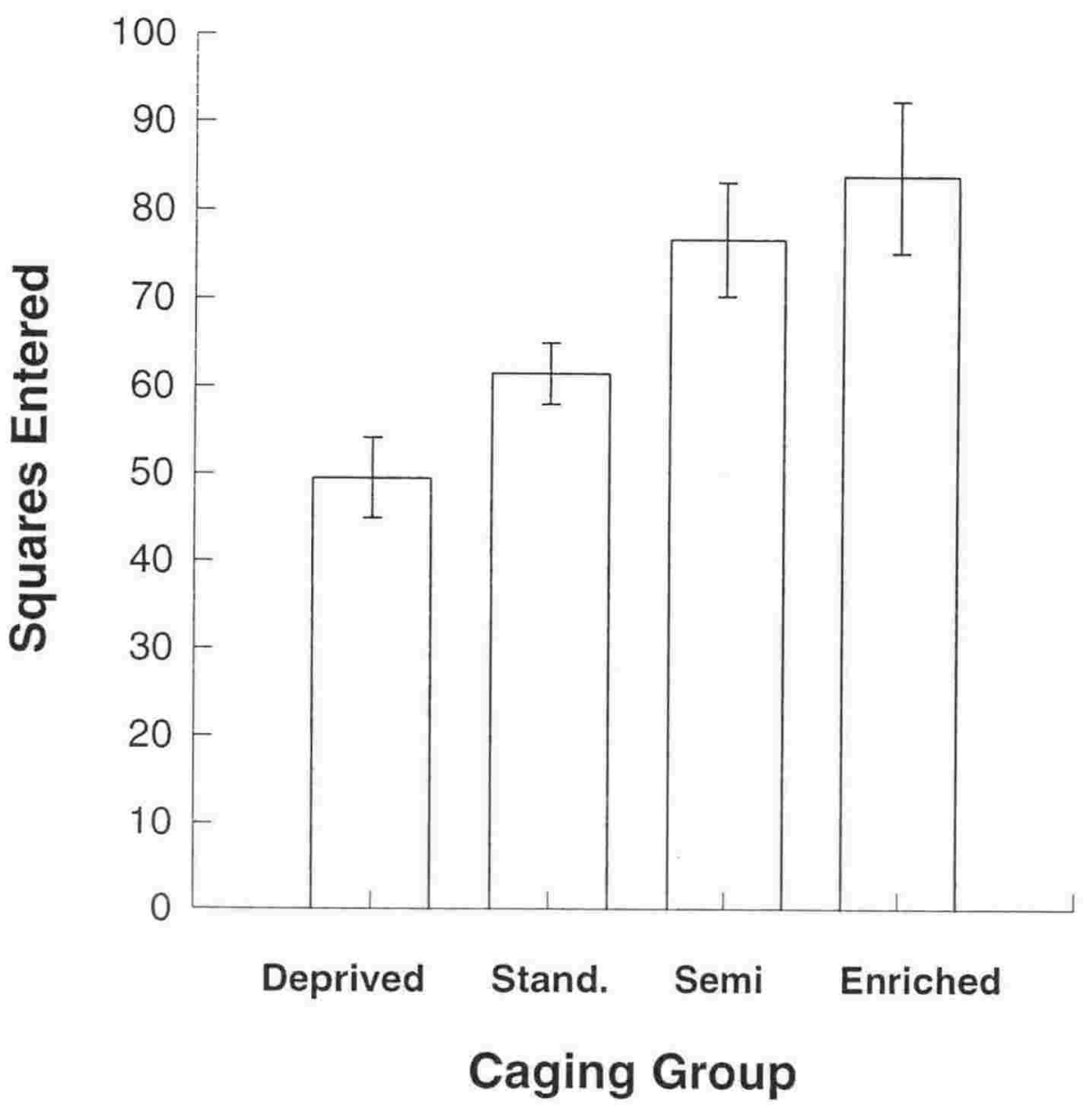

Figure 1.3 Mean number of open field squares entered during the first two minutes of the first session for deprived, standard, semi-enriched and enriched rats, (with bars showing the standard error of the mean). 


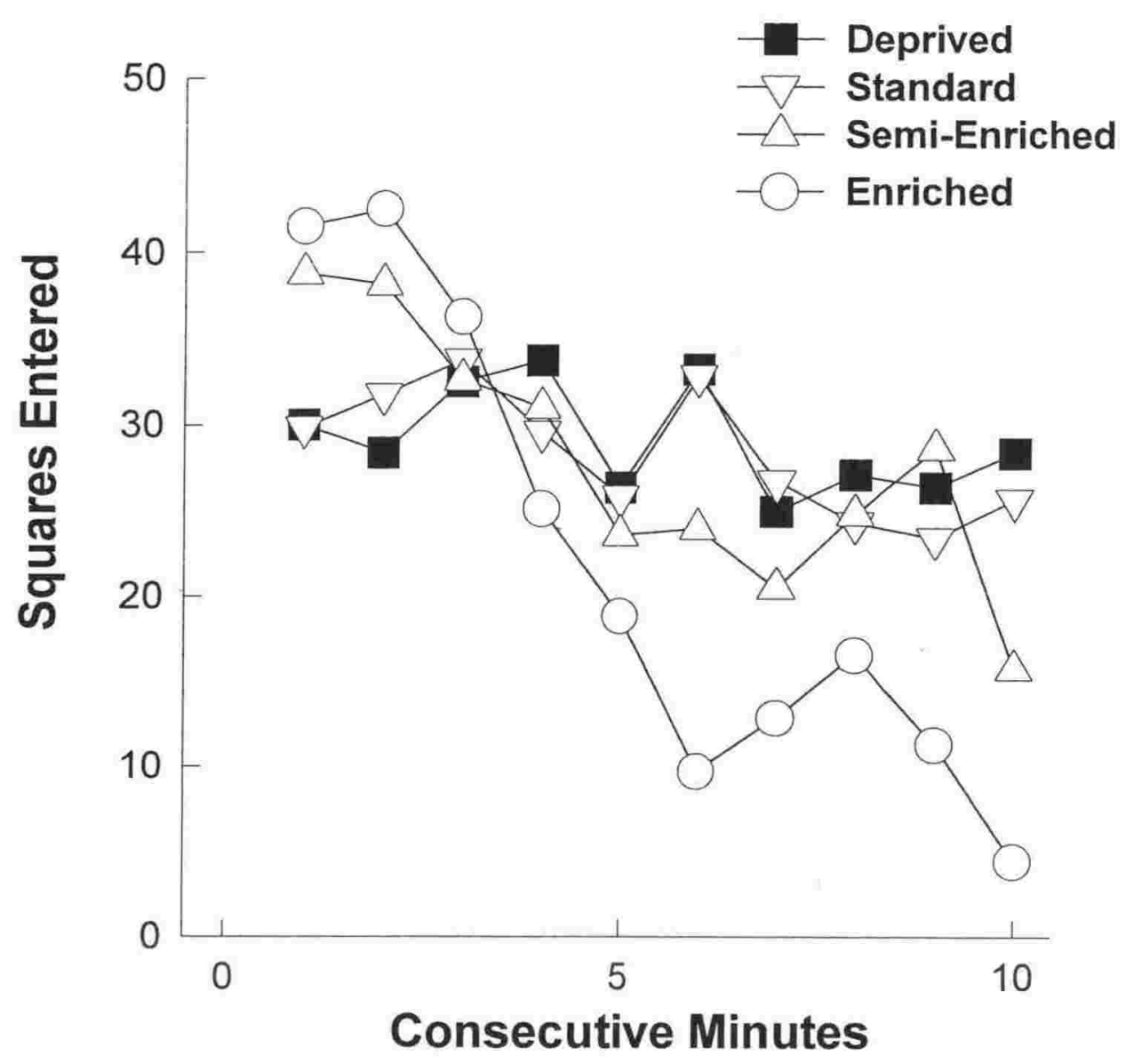

Figure 1.4 Mean open field squares entered in each minute of the ten minutes of Session 1, group means for the deprived, standard, semi-enriched and enriched rats. 
This trend was also apparent during the second and third sessions as shown in Figure 1.5. Over these two sessions there were significant group differences in total activity levels (Kruskal-Wallis, $\mathrm{H}=8.38 / 10.82, \mathrm{df}=3,34, \mathrm{p}<0.05$ ) and in activity levels for the last two minutes $(H=9.20 / 10.07, d f=3,34, p<0.05)$. A post-hoc Mann-Whitney $U$ test $(Z=-2.21$, $\mathrm{p}<0.05)$ demonstrated that this difference was the result of the low activity levels of the rats from the enriched housing condition, particularly in the second half of the sessions. (Note that one enriched rat escaped the field during all three sessions. This subject's data were included in the averages for the time that it was in the field, and excluded after its escape).

Figure 1.6 shows that there was a difference between the groups in the total and repeated Hebb William's maze errors (Kruskal-Wallis ANOVA H=11.313/13.996, df=3,35, p<0.05), but not for initial maze errors $(H=3.35, d f=3,35, p>0.05)$. A post-hoc Mann-Whitney test $(\mathrm{Z}=$ $-2.31, \mathrm{p}<0.05)$ demonstrated that the rats from enriched housing made significantly fewer total and repeated errors than any other group.

\section{Discussion}

Differential housing conditions did result in rats showing behavioural differences in the emergence box, open field and Hebb Williams maze. There was a clear difference between the behaviour of the standard rats and that of the enriched rats, so the evidence suggests that the standard conditions compromise the welfare of the rats to some extent. In terms of animal welfare the results could be interpreted in the following ways. 
- Deprived

$\checkmark$ - Standard

Semi-Enriched

- Enriched

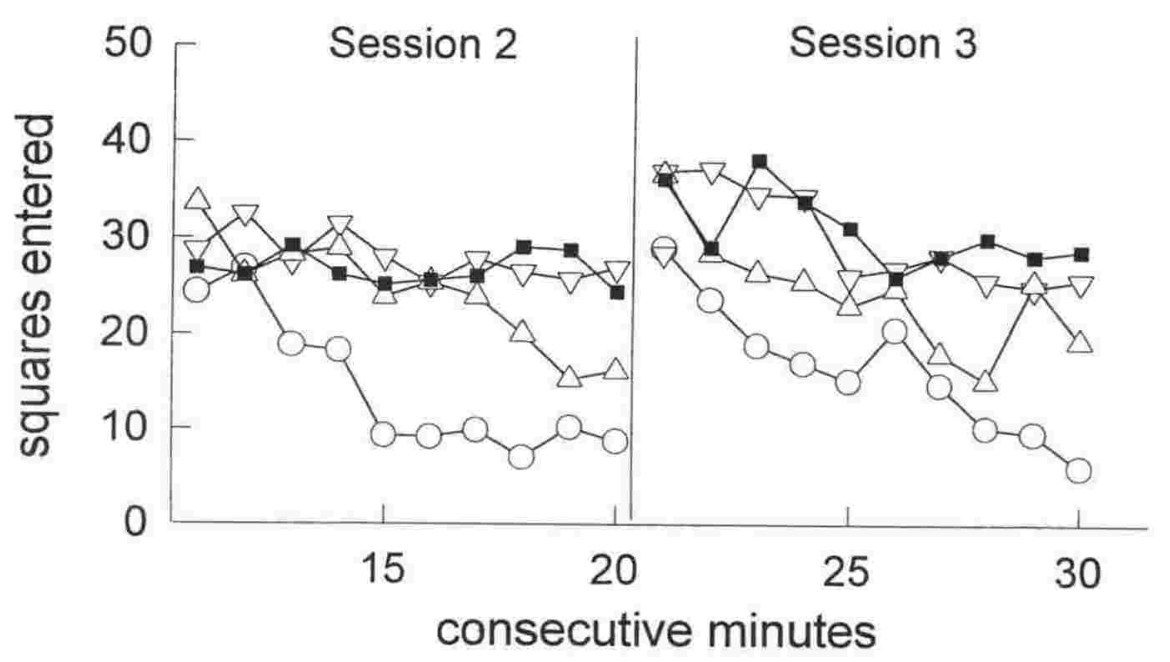

Figure 1.5 Open field squares entered as a function of consecutive minutes during Sessions Two and Three. 


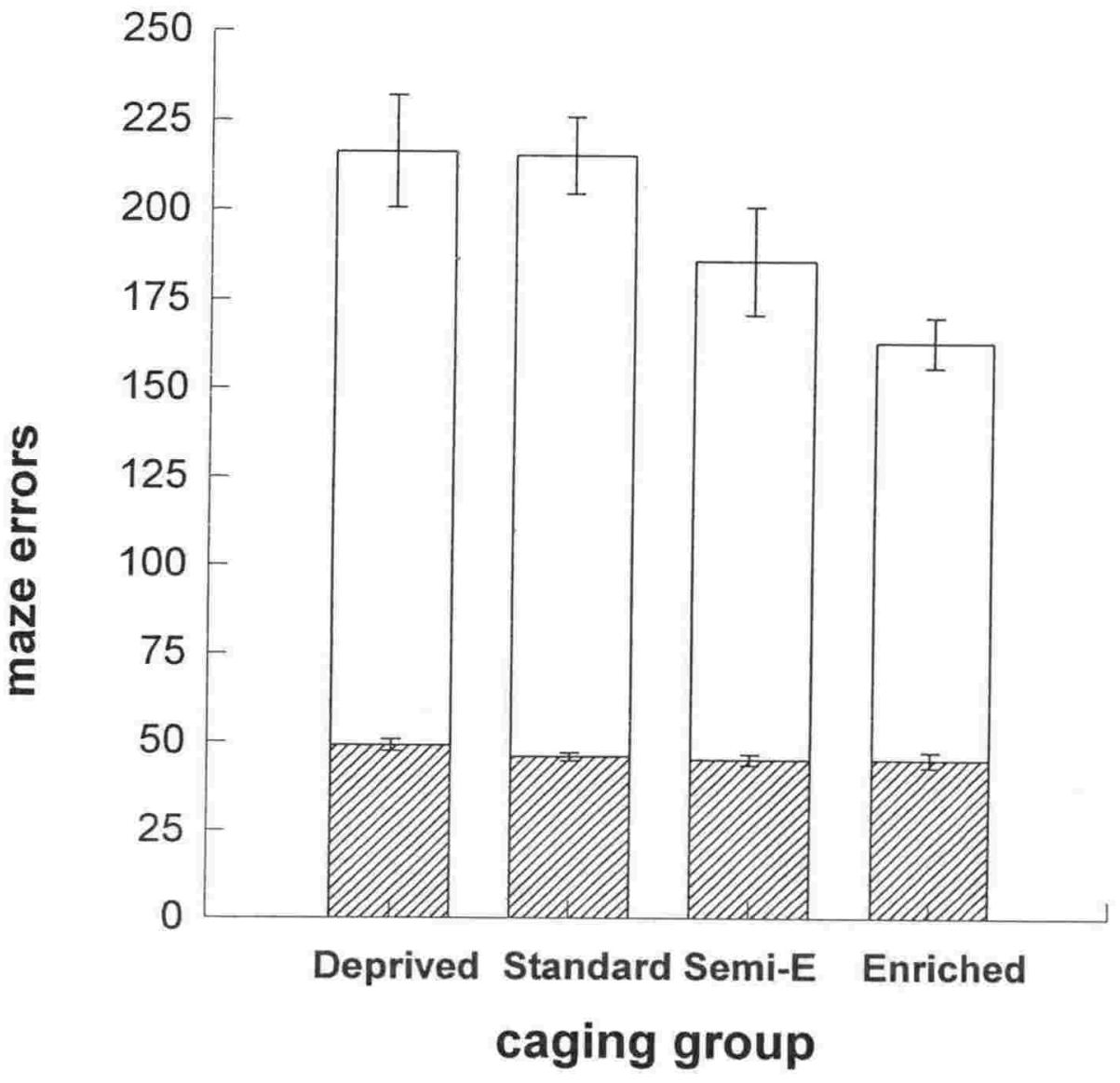

Figure 1.6 Hebb William's maze errors; total (whole bar), initial (filled portion) and repeated (white section) errors for deprived, standard, semi-enriched and enriched rats. Error bars show the standard error of the mean. 
Emergence and open field activity are both widely used indices of an animals fearfulness or emotionality. Rats that are slightly stressed, but not under attack, will become motionless in order to become harder for predators to detect. This motionless state is called 'freezing'. As a situation is made more aversive rats will freeze more frequently and for longer periods of time. On the basis of this argument the freezing group, even in their home cage, would spend more time in a state of fear than the active group does. An environment that produces rats which are more fearful than they might otherwise be, is deemed to be detrimental to their welfare.

The significantly longer emergence latencies shown by deprived rats suggest that this environment increased the time that the rats spent freezing rather than entering the novel environment. There was no such difference between the behaviour of the rats from standard and enriched environments. This suggested that standard conditions are sufficient to eliminate the fearfulness shown by deprived animals, and that enriching the environment further does not produce further improvement.

In contrast, the initial responses to the open field, which was in effect a similar neophobic reaction, showed graded differences between all of the groups. The performance of the standard group was intermediate between that of the enriched and deprived groups. It seems that the open field was a more sensitive way of coding freezing, while the emergence score produced an 'all or none' type of response and does not reflect the intermediate levels of responsiveness very well. 
The current ten minute session length was chosen in order to investigate the effect of enrichment on habituation rates. Archer (1973) suggested that the rate at which a rat adapts to the environment is an important aspect of its neophobic responsiveness which is often neglected in favour of measures of over-all levels of activity. The enriched rats immediate high levels of exploration quickly led to them becoming inactive and showing resting behaviour. This rapid sequence of behaviours could be interpreted as a superior rate of settling in or adapting to the change of environment. The standard and deprived groups maintain their levels of responsiveness during all three sessions, suggesting a more chronic fearful state.

It is possible to argue that the deprived rats are not failing to habituate, but merely more novelty-seeking and thus they explore longer. However this possibility is inconsistent with the deprived groups initial freezing, or their failure to ever reach a high level of activity,

Previous studies that used the open field have found a range of results, largely due to the fact that there is no standardisation of equipment or procedures between laboratories. Although many variables may be contributing to this variability the current data suggests that session length might be one of the most important. Researchers who have reported enriched rats as the most active group often used short sessions; Ader (1965) for example, used a two minute session and Holson (1987) used three minute sessions. Brown (1968) found no group differences when a single session length of five minutes was used. Studies that found deprived rats were more active have often used longer exposure times; (e.g. Morgan, 1972; ten minutes; Smith, 1972, fifteen minutes). The tendency for researchers to collect data as 
sums over varying session lengths may account for a lot of the disagreement in the literature.

Indeed, in the current study, averaging activity levels over the ten minute total time, made activity levels seem similar across the groups when they actually differed both in initial activity and habituation rates. Only one experiment did not fit this trend; Gill et. al. (1966) found enriched rats were more active using a ten minute session. This might be due to the influence of one of the other uncontrolled (and often unreported) variables, such as lighting, wall height, field shape and size, method of subject transportation, and so on.

The Hebb William's maze is an animal 'intelligence' test of long standing use. It provides very reliable results and is frequently used to assess the cognitive effects of drugs or brain damage (Rose, 1988). In this context a poor maze score is thought to reflect a cognitive deficit relating to memory or learning.

On the Hebb William's maze task, the enriched rats significantly out-performed the rats from other conditions. On average the semi-enriched rats performed better than those from deprived or standard housing. This result suggested that rats kept under standard conditions may experience a deficit similar to that of deprived rats. This result suggests that behavioural laboratories may be studying complex behaviours, such as memory, with animals whose ability to perform these behaviours is impaired. Housing conditions must be considered a significant variable in the area of behavioural research.

This study used two methods for delivering enrichment to laboratory rats. The first was a large scale enrichment provided for a short period after weaning. The current study 
demonstrated that this enrichment has a significant effect on rats' behaviour. However the full enrichment may be impractical to provide for long periods of time, and it is uncertain how long these benefits would persist after the rats returned to standard conditions.

The other method was a more moderate form of enrichment as part of the rat's normal housing. The current 'semi' enrichment was only mildly effective. However this does not invalidate the over-all approach. The result does indicate that further research is needed into how to construct an effective long term enriched environment, and to investigate how long lasting its effect might be.

Currently very little research about rats environmental needs exists. The enriched condition was constructed according to 'educated guesses' and does not control for, or attempt to measure the effect of any particular dimension (such as manipulatable objects or stocking density). As such, it is impossible to determine what aspects of the enrichment are effective, or to what extent. If the key elements of enrichment were known, then moderate forms of enrichment could be made more effective, and the need for more unwieldy enrichment conditions would be reduced. After establishing that the cage is in need of improvement, different interventions could be assessed using preference or economic demand procedures (Hursh, 1980; 1984).

In summary, results from the emergence task suggests that standard conditions may be sufficient to alleviate 'fearfulness'. However, results from the open field test showed that standard rats were on average slightly more active than deprived rats, while semi-enriched and enriched rats were significantly more active and show rapid habituation of activity levels. 
Thus, the fearfulness of standard rats could be further reduced by more enriched housing conditions. Keeping an animal in a cage that makes it more sensitive to stressors is equivalent to increasing the stressors themselves.

The problem solving ability (as measured by the Hebb William's maze) of rats kept in the enriched housing was superior to other groups. This implies that the standard environment was not sufficiently stimulating for these abilities to develop optimally. Rats with these deficits may have more difficulties in day-to-day learning situations. As such, the enriched environment seems to be preferable in terms of welfare, and more efficient ways of delivering this enrichment need to be investigated.

Although there is enough evidence to proceed with a programme of improvement of the standard cage, there is also significant doubt about the validity of some of the findings. The Hebb William's maze is a widely used piece of equipment. Its long history and popularity has however always been balanced by a minority who doubted that the behaviour measured was of a 'higher' cognitive ability, equitable with intelligence, rather than of emotional or motivational or basic perceptual abilities (for example, Zimbardo \& Montgomery, 1960). There is indirect evidence both for and against the validity of the old intelligence test, but the concerns have never been properly laid to rest.

The Hebb William's maze continues to be used, especially in pharmacological research (for example; Shibagaki et. al., 1981). While the implications of the maze results are widely accepted, often without a proper appreciation of their origins. The study of animal welfare must be accompanied by constant re-evaluation of the procedures we use in this process. The 
following experiments have these two goals in mind not only for the Hebb-William's maze, but with all of the procedures used in this thesis. 


\section{Chapter 2:}

\section{The Cognitive Deficit}

Experiment 1 suggested that deprived caging affects not only fear levels, but also the cognitive abilities of rats. However, there are doubts about the use of the Hebb William's maze to assess cognitive performance.

The present study aimed to find the cognitive deficit that the Hebb-William's maze purports to measure, by using procedures other than the Hebb-William's maze. Finding such an effect might support the validity of the maze and it would justify the widely held (but weakly supported) belief that such a deficit exists. Also, the exact nature of such a deficit might be elucidated by the use of a modern test of a more specific range of cognitive abilities. What remains to be decided is, what specific cognitive abilities might the Hebb William's maze performance depend on? As demonstrated in Experiment 1, the deprived rats have a greater tendency to repeat an error after they have made it, compared with enriched rats. This suggests that the deficit identified by the Hebb William's maze may be a memory difference between enriched and deprived rats (Experiment 2.1). However, this chapter also investigated persistence (Experiment 2.2) and learning (Experiment 2.3) as two other cognitive abilities that Hebb William's maze performance could plausibly reflect. 


\section{EXPERIMENT 2.1}

\section{Memory}

The Hebb William's maze was originally described as measuring 'animal intelligence' (Hebb, 1947) and 'problem-solving' (e.g. Denenberg et. al., 1968). These terms are fairly loosely defined, and there is no independent evidence to suggest that maze scores really reflect this kind of general cognitive competence. Examination of the data in Experiment 1 and other studies suggests that the key behaviour is the repetition of mistakes. Maze errors can be divided into 'initial' errors when a blind alley is entered for the first time, and 'repeated' errors when the same alley is re-entered before the end of the session. Enriched and deprived rats made the same number of initial errors and differ only in terms of their repeated errors, specifically - the deprived rats made more repeated errors. This suggested a number of possible cognitive explanations. The first of these is that the deprived rats do not remember their actions during the previous seconds or minutes. That is that they enter the dead end because they do not recognise it from earlier in that trial or from previous trials in the same session.

A common modern method for assessing memory in non-human animals is called Delayed Matching to Sample (DMTS; Blough, 1959; White \& Alsop, 1993). Each trial in this procedure is made up of three steps. First, the subject is presented with one stimulus. Second, there is a delay period when no stimuli are presented. Finally, two stimuli are presented including the stimulus from step one. Each of these stimuli are associated with a response option. If the animal responds to the stimulus presented in stage one it receives reinforcement. If it responds to the other stimulus there is a time-out period. 
Following the time-out or reinforcement period there is a brief interval, and then the next trial begins. Care is taken to provide different trials which vary the stimulus that is correct and the position of the correct stimulus. With rats, a variation of this procedure is often adopted, called Delayed Non-Matching to Sample (DNMTS). DNMTS provides reinforcement in step three for responding to the stimulus that was not seen in step one. Rats are believed to learn DNMTS more readily than DMTS.

Two basic kinds of apparatus that can be used with the DNMTS procedure are an enclosed maze and an experimental response chamber. The following experiment utilised an enclosed maze (a T-maze) in Part 2.1.1 and an automated experimental chamber in Part 2.1.2. The advantage of the T-maze was that it is similar to the Hebb William's maze in terms of the response requirement and stimuli used. The fully enclosed and automated chamber had the advantage of producing a uniformity of stimulus and manipulanda presentation that a human maze operator could not provide. Both the T-maze and the chamber have advantages over the Hebb William's maze because they do not incorporate open or expansive areas, and so should minimise any exploration based differences. Thus, if a genuine memory difference exists between enriched and deprived rats, it should be apparent as measured by these methods.

Another factor, the period of deprived housing, was introduced into the first part of the experiment (2.1.1: The T-maze). There is some inconsistency in the literature as to whether the rats remain in their housing conditions throughout an experiment (for example Woods et. al., 1961), or are rehoused into a more moderate condition (for example, Krech et. al., 1966). While re-housing in more restrictive conditions generally has very little effect on enriched rats, improvements in condition can have marked effects on deprived rats (Krech, 
Rosenzweig \& Bennett, 1962). If a memory deficit was detected it would be interesting to assess the relative contribution of pre-experimental housing versus housing during the experiment. One group of (6) deprived rats was rehoused under standard conditions after thirty days, and the other deprived group remained in deprived caging throughout the experiment. As the re-housed and non-rehoused deprived subjects were kept at different times, two separate groups of re-housed enriched groups were provided for comparison purposes. Re-housing was not practised with the enriched rats because previous literature has indicated that re-housing had little effect on enriched rats (Krech et. al., 1962). In addition, the configuration of the enriched cage made it hard to identify and remove specific animals without disturbing them much more than the deprived rats would be disturbed by the same manipulation.

\section{Method}

\section{Subjects}

The subjects were 36 female Hooded Norway rats (24 in Part 2.1.1, 12 in Part 2.1.2) that were $60(+/-2)$ days old at the beginning of the experiment.

\section{Apparatus}

Part 2.1.1: The T-Maze

The enriched and deprived housing is described in Experiment 1. The T-maze was wooden, with an arm width and height of $100 \mathrm{~mm}$. The length of the start arm and choice arms were 800 and $600 \mathrm{~mm}$ respectively. 


\section{Part 2.1.2: The Experimental Chamber}

The chamber had two retractable levers on the front wall. There was a $12 \mathrm{~W}$ light above each lever and a condensed milk dispenser between the two levers. The milk dipper provided 0.1 $\mathrm{ml}$ of sweetened condensed milk diluted with three parts of water. There was also a fixed lever on the opposite wall. The chamber was $20 \times 25 \times 20 \mathrm{~cm}$ in size with a grid floor over wood shavings.

\section{Procedure}

Half of the rats were assigned to an enriched condition and half were assigned to a deprived condition. All of the enriched rats and six of the deprived rats from Part 2.1.1 were rehoused into standard conditions at the beginning of the experiment. The remaining deprived rats were left in the deprived environment throughout the experiment. The enriched, deprived and standard conditions were as described in Experiment 1.

\section{Part 2.1.1: The T-Maze}

Maze testing occured between 10am and 3pm with the order counterbalanced for housing group, but constant across days. The animals were all fed after the last rat had run at 3.30$4 \mathrm{pm}$.

The rats were habituated in the maze for one session. This session ended when they had eaten at least ten chocolate chips while in the maze. In the next session they were placed in the start arm and the experimenter waited until they had run to the end of a choice arm and taken a chocolate chip placed at the end of that arm (this was repeated at least ten times). Next each subject was introduced to the experimental procedure. Trials were conducted in 
pairs with no delay between the two trials in a pair but a 30 second delay between pairs. On the first trial of each pair one arm was blocked and the other was baited with chocolate. On the second trial of each pair both arms were open but only the previously blocked arm was baited. Thus a correct choice was to go to the arm not baited in trial one. Rats were given 20 pairs of trials per session until they achieved an accuracy of $9 / 10$ for five consecutive blocks of ten trial pairs.

In the memory testing phase trial pairs were still separated by 30 seconds but the delay between the two trials within a given pair changed to equal numbers of $0,1,3$ and 5 minute delays. The baited side (left or right) and delay length on successive pairs was determined according to a quasi-random sequence with a maximum of three consecutive trials that were the same on either variable. Training continued for 23 sessions ( 460 trial pairs per rat) by which time over-all-accuracy was visually stable.

\section{Part 2.1.2: The Experimental Chamber}

The rats were maintained at approximately $80 \%$ of their free-feeding weight by restricting feeding to a one hour period at the end of the day. Restriction was by time rather than weight as most of the animals were not fully grown at the beginning of the experiment. Sessions were conducted for 50 minutes a day in six sessions between about 9 am and $3 \mathrm{pm}$, and fed between 10 and 60 minutes after completing their session. Rats were auto-shaped to respond on the front lever for reinforcers.

The rats were then trained on a DNMTS task until their performance was stable. The DNMTS programme involved trials separated by unlit inter-trial intervals of 30 seconds. At 
the beginning of each trial one of the levers was extended. Immediately after the delay had timed out, the first response on the back lever resulted in both levers being inserted into the chamber. If the rat pressed the lever that was not previously extended at the start of the trial, it was rewarded with 3 seconds access to sweetened condensed milk. Otherwise it was not reinforced and the 30 second interval began immediately. Each session lasted for 40 minutes and occurred daily.

The rats were trained with a 0 second delay, before being tested with $0,5,10$ and 20 second delays. Each session contained equal numbers of each trial type. The order of delay length and side of the sample lever were random with the restriction that no more than three consecutive trials had the same delay or the same correct side choice. A subsequent set of trials was conducted with $0,5,10,20$ and 40 second delays. The data analysed were from day 32 to 37 for the first set of delays, and from day 15 to day 20 for the second set. The five data collection days began when overall performance levels were visually stable.

\section{Results}

The individual raw data (number correct) for this experiment are shown in Appendix 2.1.

\section{Part 2.1.1: The T-Maze}

Error rates were stable across all 23 sessions that included delays greater than zero. So data from all of these sessions were included in the analysis (115 trials at each delay). Each subject's data was reduced to one score (percent correct) for each delay. 
The top graph in Figure 2.1 shows the mean percent correct for each housing condition. There is no apparent difference between the deprived rats that were rehoused under standard conditions (1) and those that remained in deprived conditions during testing (2). Nor is there any clear difference between the two enriched groups that were tested at these separate times (1 and 2). For this reason, all 24 subjects were used to assess the effect of the other variable housing condition.

The lower graph in Figure 2.1 shows the mean accuracy (percent correct) for the enriched and deprived groups. The enriched rats showed superior performance at all delays. A repeated measures ANOVA found a main effect for delay $(\mathrm{F}=204.15, \mathrm{df}=3,66, \mathrm{p}<0.05)$ and group $(\mathrm{F}=6.17, \mathrm{df}=1,22, \mathrm{p}<0.05)$, but no interaction $(\mathrm{F}=1.09, \mathrm{df}=3,66, \mathrm{p}>0.05)$. For both groups accuracy decreased as delay increased and the enriched rats were more accurate than the deprived rats at all delays, including zero. However, if the differences between the enriched and deprived rats was purely memorial the enriched rats superiority would increase as delay increased, which would be reflected in a significant interaction. This was not the case.

\section{Part 2.1.2: The Experimental Chamber}

The automated data collection supported the use of a more sophisticated measure of accuracy in this part of the experiment. The measure used $(\log d)$ was calculated according to Equation 2.1 below.

\section{Equation 2.1}

$$
\log d=0.5 x((\log (\text { correct left/error left }) x(\text { correct right/error right }))
$$



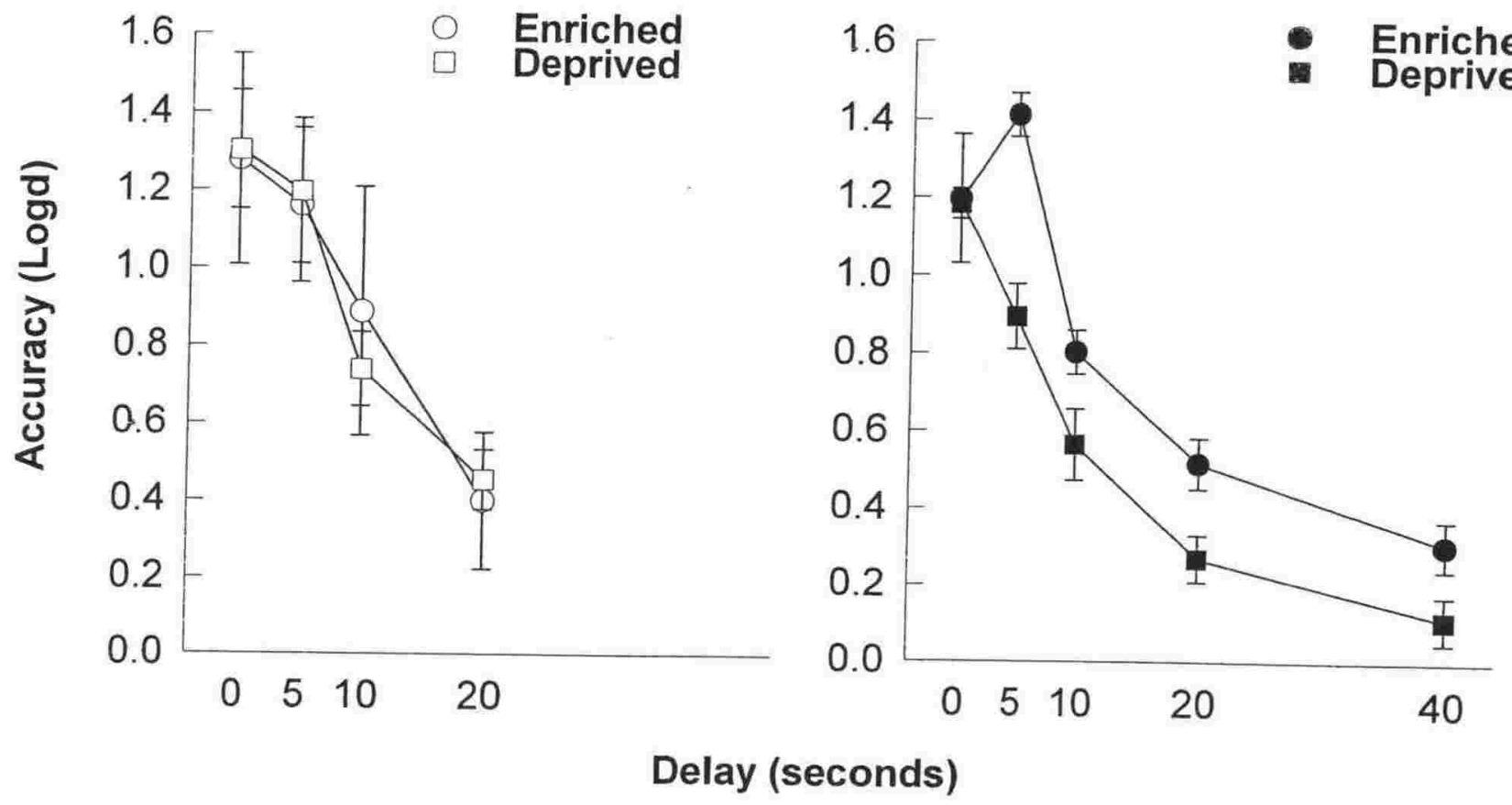

Figure 2.1 Percent correct responses as a function of inter-trial delay, for rats from deprived and enriched caging (with standard error bars). The top graph shows the two sets of enriched and deprived groups, which are combined in the bottom graph. 
A correct was a response to the lever not presented during step one of that trial, and an error was a response that matched the lever shown in step one (remembering that this was a non-matching to sample task). Left are trials when responding to the left lever would result in food reinforcement (and right when a response to the right lever would be reinforced). This equation gives a measure of accuracy which is not affected by any tendency of the subject to respond according to a side bias independent of ability to discriminate between levers (e.g. to the left lever). Any such position bias is quantified by the following equation.

\section{Equation 2.2}

Bias $=0.5 x \log (($ correct left/error left $) x($ error right/correct right $))$

Davison \& Tustin (1978).

The bias estimates from the current data were small and consistent across all delays, allowing the following analysis to concentrate on the $\log d$ (accuracy) parameter. Figure 2.2 shows the mean $\log d$ values for both enriched and deprived rats. The first graph includes data from the sessions that included delays up to 20 seconds. In this case there was no apparent difference between the performance of the enriched and deprived rats. A mixed design ANOVA showed that $\log d$ was significantly affected by delay $(\mathrm{F}=31.31, \mathrm{df}=3,30, \mathrm{p}<0.05)$, but there was no effect of housing condition on the degree of impairment produced by these delays $(F=0.00, d f=3,30, p>0.05)$ and no interaction between these two variables $(F=0.45, d f=3,30$, $\mathrm{p}>0.05)$.

The second graph (Figure 2.2) summarises sessions that included a 40 second delay. In this case the enriched rats appeared to perform more accurately than the deprived rats. This is 

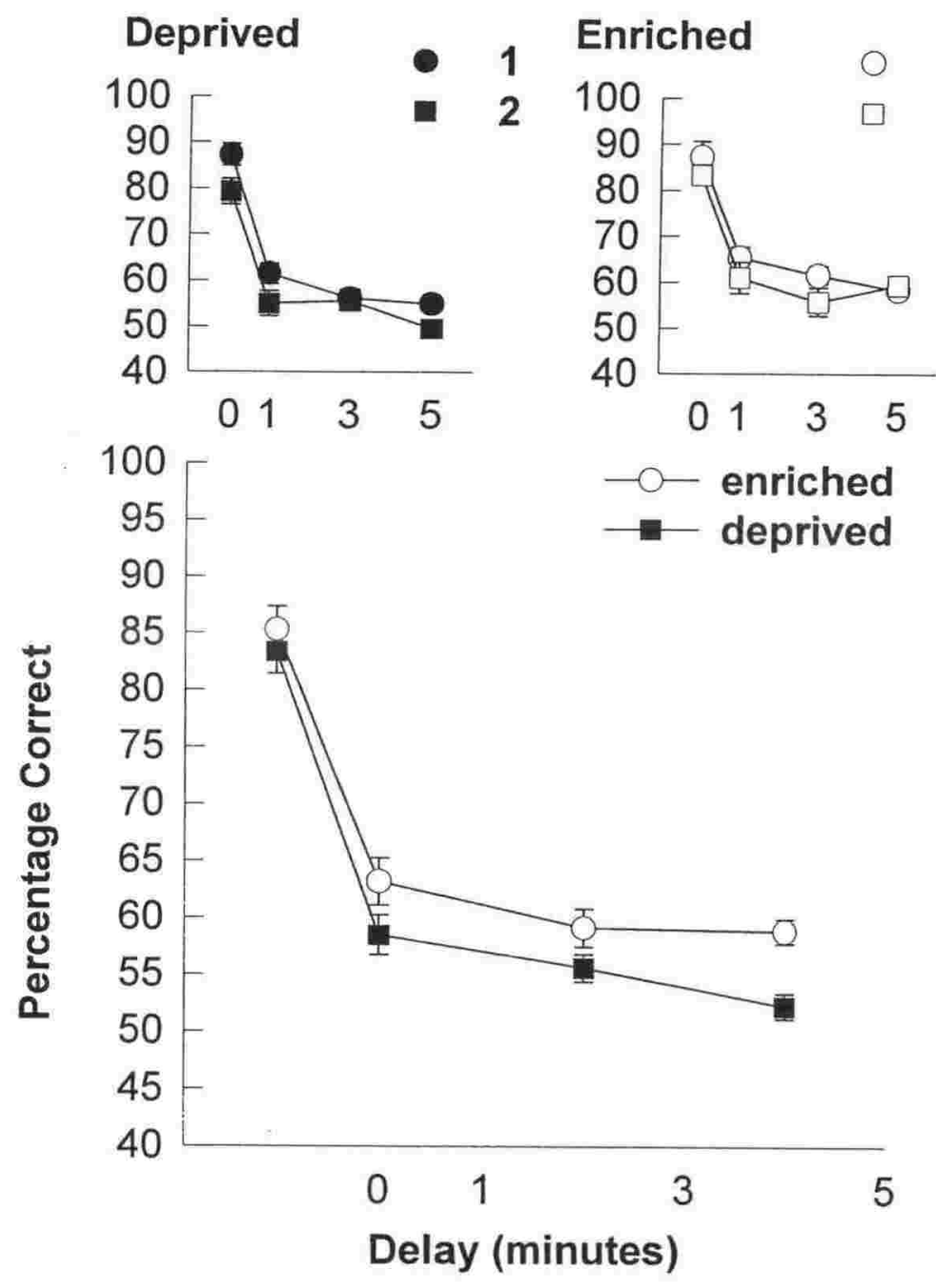

$\begin{array}{lll}\text { Enriched } & & \\ 100 & & \mathbf{1} \\ 90 & \square & \mathbf{2}\end{array}$

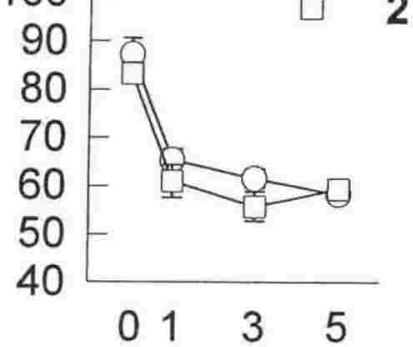

-O- enriched

$$
2
$$


reflected in a mixed design ANOVA with two significant main effects for delay $(F=62.86$, $\mathrm{df}=4,40, \mathrm{p}<0.05)$ and housing condition $(\mathrm{F}=20.67, \mathrm{df}=1,10, \mathrm{p}<0.05)$. However, there was again no interaction between delay length and housing condition $(F=2.64, d f=4,40, p>0.05)$, indicating that the difference between groups was not based in memorial processes.

\section{Discussion}

The results of this experiment do not provide any compelling evidence that caging environment affects the memory of rats. The T-maze data suggested that the enriched rats have a small but statistically significant advantage at all delays (including zero). The fact that this advantage was not dependent on delay length shows that it is not a superiority of memory. It appears that the enriched rats are more able to perform the discrimination, and thus they may have a superior perceptual or attentional ability than the deprived rats. Given that the left and right choices used as stimuli would arguably be easy to distinguish, differences in attention offer the most convincing description of the data. This kind of ability is independent of memory, as it is measured by the relative decay of performance over time (White, 1985).

Data from the experimental chamber found no effect of housing on performance on delays of up to 20 seconds. However, when the task was made more difficult (by adding a 40 second delay) the enriched rats showed more accurate performance than the deprived rats. This finding was consistent with the T-maze data in that the difference between enriched and deprived rats occurred at all delays and so was not due to differences in rates of forgetting. 
When the 40 second delay was added to the operant procedure the deprived rats performance on the 0,510 and 20 second delays fell below that of the enriched rats who maintained their accuracy at these delays. This suggests that the deprived rats were able to encode visual discriminations as well as enriched rats (at least at delays of 0-20 s), but this performance was sensitive to disruption by changes in the procedure. It is not clear how the addition of the 40 second delay could have disrupted the deprived subjects performance, it may have been that the disruption occurred because the task became more difficult, or due to the drop in overall reinforcer rate when a longer delay was added. Both of these would lead to an overall decrease in accuracy in normal subjects (White, 1985). If the change in the reinforcer density was implicated, the deprived rats response suggests that they are more sensitive to reinforcement than the enriched rats. The effect of housing condition on T-maze performance might also reflect the deprived rats being more easily distracted from the relevant stimuli, as the T-maze involves exposure to handling, being tested in the same room as other subjects and other possible disturbances.

It would be interesting to repeat this experiment with the 40 second delay included from the outset, to see whether this would produce equivalent performance between enriched and deprived rats at this delay also. At this point it is still possible that the deprived rats would show a deficit at this delay even under optimal conditions (i.e. this difference could still be due to differences in rates of forgetting).

Regardless of the implications for attentional differences between the two groups there is no convincing evidence that enriched and deprived rats differed in their memory performance. The lack of memorial differences suggests that if cognitive differences are present in deprived 
rats they are differences other than memory. If an animal repeats its errors, but does not have an impaired memory, there are other possible cognitive explanations. 'Response perseveration' was identified by Van Haaren and van Hest (1989) as a description of an animal's tendency to repeat errors. This possibility was examined in Experiment 2.2. 


\section{EXPERIMENT 2.2:}

\section{Persistence}

Experiment 2.1 ostensibly assessed memory performance, but the effect of delay on accuracy did not differ across groups. The crucial aspect of the DNMTS task in this case may be the simple visual discrimination that the procedure is based on. It was argued that the data could be interpreted as showing that the enriched rats persistently attended to the discrimination, but the deprived rats were more easily disturbed by the less automated maze method of testing or by changes to the operant procedure.

On the other hand, a range of indirect evidence suggests that deprived rats typically respond more persistently than enriched rats in a range of experimental contexts. Luchins and Forgus (1955) used a specially designed maze to demonstrate the deprived rat's 'behavioural inflexibility' in terms of the path they follow through the maze and how slowly they change this path in response to obstacles. Deprived rats are also resistant to extinction (of bar pressing behaviour) in an experimental chamber (Davenport et. al., 1976), their lever responses were less affected by disrupters (Jones et. al., 1991) and they take more time to change food obtaining strategies (Morgan, 1973).

Most of the studies cited above are isolated cases and given the possible effects of emotional confounds their meaning is uncertain. However, two findings consistent with the idea that deprived rats are more persistent, are more reliable. One is the finding that deprived rats make more repeated errors than enriched rats on the Hebb William's maze (Experiment 1 for 
example). The other is that deprived rats perform poorly on visual discrimination reversals but not initial discrimination learning (Krech et. al., 1962; Jones et. al., 1991).

In addition, from a theoretical perspective, it could be argued that the more diverse early experience of enriched rats might have increased their ability to swap from task to task and seek out alternative sources of reinforcement, whilst the deprived rats have had few sources of reinforcement other than food and so might be more difficult to distract from a foodrewarded task. This difference in reinforcement history would suggest the deprived rats would be more persistent.

On the other hand, differences in persistence are not the only possible explanation of the existing data. The deprived rats may have a perceptual deficit which stops them from using extra maze cues to distinguish between the correct path and a dead end. In the absence of extra maze cues they may then confuse paths and repeat their errors. Alternatively, the enriched rats advantage on a discrimination reversal task might be due to over-training. The longer an animal successfully performs a task the more flexible it will be (Macintosh, 1962). As the enriched rats learn more quickly they would spend more time within a condition where their performance showed high levels of accuracy, and this in turn could produce more flexible behaviour. If this were so, the enrichment effect would be on learning rate and only indirectly on persistence. The evidence is currently too variable and too thinly spread to differentiate between these possible explanations.

In order to evaluate the nature and extent of any genuine differences in flexibility between enriched and deprived rats, we need to be able to measure persistence directly. One 
procedure that quantifies behaviours of this type has been outlined by Tony Nevin in experiments relating to his 'Behavioural Momentum Theory' (Nevin, 1992). There is a concept in this theory termed resistance to change which is very similar to persistence.

Resistance is normally tested experimentally in a response chamber. An animal is shaped to press a lever or key, and then responds on intermittent schedules (usually a multiple variable interval schedule), until their rate of responding is stable. Then some form of disruption occurs and change in behaviour is then measured as a proportion of the baseline response rate.

There are two independent variables that are used in this type of experiment. One is the type of disrupter used. Previously used examples are extinction of reinforcement (Nevin, 1974), free food (Nevin, 1974) or drugs (Cohen, 1986). The other independent variable is the reinforcement rate in the two schedules provided within the experimental session. Behaviour in the presence of the schedule that provides more frequent or larger reinforcement is normally more resistant to change proportional to its pre-disruption baseline level, than behaviour shown in the presence of the other schedule (see Nevin, 1992, for a review).

This experiment introduces a third independent variable in the form of the previous housing experience of the subjects. There is a definite potential for addressing the effect of preexperimental manipulations of the environment using the behavioural momentum methodology. In this case all other experimental conditions would be held constant, and performance of enriched versus deprived groups would be compared in terms of the relative proportion of baseline responding maintained in the presence of disrupters. 
The following experiment used extinction, pre-feeding and the presence of novel objects as disruptive stimuli. The data will be average across both schedules to compare the relative resistance to change exhibit by enriched and deprived rats in the presence of these stimuli. If the deprived rats are more persistent then they should be less distracted by the disrupters and maintain a higher level of responding (compared with baseline). Different types of distracter were used so that the results could not be attributed to any specific aspect or types of intervention, rather than the overall distracting role. Response rates will also be compared between the rich (VI30 s), and lean (VI240 s) schedule. Behaviour in the presence of the VI30 s schedule should be more resistant to change, replicating this finding would help to suggest that the behaviour being measured was indeed resistance to change.

\section{Method}

\section{$\underline{\text { Subjects }}$}

The subjects were two groups of 12 female Hooded Norway rats. Each group consisted of 6 enriched and 6 deprived subjects. Six of the deprived rats remained in deprived conditions, but all other subjects were rehoused under standard conditions at the beginning of the experiment. They were approximately 60 days old at the beginning of this experiment.

\section{Apparatus}

Two operant chambers similar to those used in Experiment 2.1.2 were used. They differed in having only one retractable lever on the front wall, and no levers on the back wall. A pool of small objects were used in three of the conditions. For the one-object condition the object 
was a ping pong ball, the two object condition used a wooden block $(2 \times 5 \times 10 \mathrm{~cm})$ and a plastic pottle (plastic with an open top of $10 \mathrm{~cm}$ diameter, $8 \mathrm{~cm}$ diameter base and $12 \mathrm{~cm}$ in height) and for the three object condition, paper tissue, a curtain ring and a bulldog clip.

\section{$\underline{\text { Procedure }}$}

The rehoused (Group 2.2.1) and non rehoused (Group 2.2.2.) subjects were differentiated as described in Experiment 2.1. At the commencement of training the rats were auto-shaped to press a lever and they then spent two sessions on a VI30 s schedule. Finally the rats were exposed to a multiple VI30 s VI240 s schedule. Components alternated every 60 seconds and were signalled by illuminating or turning off the light above the lever. The schedule that occurred first, and the component signalled by the light, were counter-balanced across rats.

After the response rates were stable (judged visually by agreement of three experimenters after 37 sessions) probe disruption sessions began. Probe sessions occurred with at least five days in between each, to obtain an estimate of baseline response rates. The disrupters were pre-feeding (6-15ml of reinforcer), novel objects (1-3 objects) and extinction ( 3 consecutive days). The subjects were pre-fed in their home cage 10 minutes prior to the session with the same sweetened condensed milk used as a within session reinforcer. Twelve identical objects of each type were used on the novel objects trials. The objects were washed and dried, and then placed in the operant chamber approximately ten seconds before the session began. Extinction involved the subject being run as usual with the difference that responses on the lever produced no food or food-hopper movement. 


\section{Results}

The effect of housing on resistance to change was calculated by averaging the response rate of the subject for the five days prior to the probe (disrupted) session and across both of the schedules. This baseline response rate is designated 1.0, and responding during the probe trial is calculated as a proportion of that 1.0. For example if the subject's response rate doubled this would be a 2.0 , if it halved this would be a 0.5 . These values were then plotted on a $\log _{10}$ scale as shown in Figures $2.3,2.4,2.5$ and 2.6.

Figure 2.3 shows the relative resistance to change of behaviour maintained on the two schedules. The dark circles show proportion of baseline response rate shown on the VI30s, and the white circles the VI240 s. With pre-feeding the richer schedule was slightly more resistant to change, as shown by the dark circles being, on average, above the white circles. However, this pattern was small and not statistically significant (mixed design ANOVA F values were between 0.36 and 1.62 depending on the disrupter, $\mathrm{P}>0.05)$. In the remainder of the figures in this experiment performance on both VI schedules was averaged in order to investigate the effect of housing condition.

Figures $2.4,2.5$ and 2.6 show the $\log$ proportion of baseline responding shown by the rats under the influence of the three disrupters; pre-feeding, novel objects and extinction respectively. The three graphs that make up each figure show performance of the group including the rehoused deprived rats, the group with the non-rehoused deprived group and both groups combined. The group whose data is higher, or whose data has the shallowest 
- VI $30 \mathrm{~s}$

O VI $240 \mathrm{~s}$

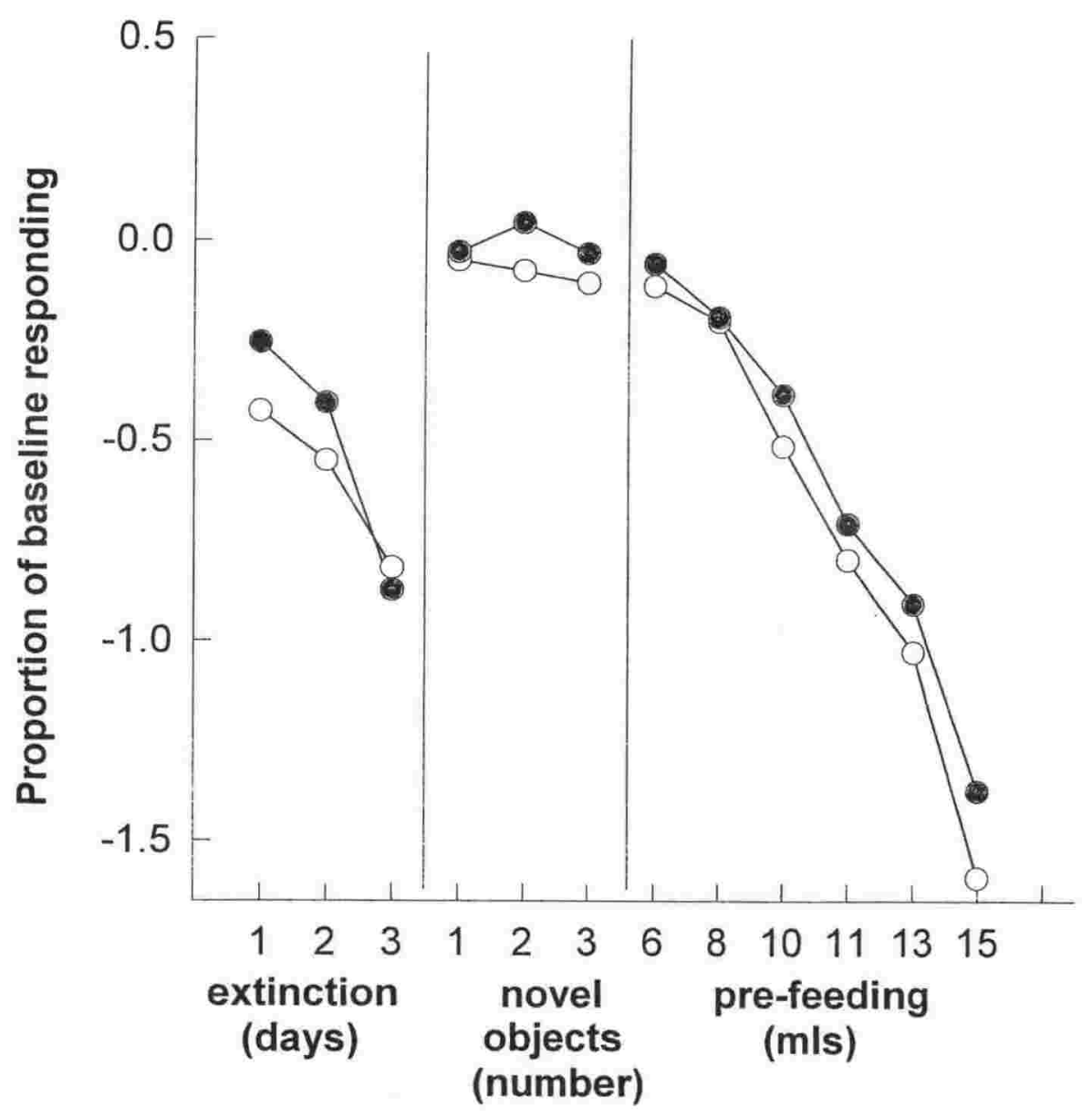

Figure 2.3 Resistance to change as a function of disrupter and schedule component (VI240 s versus VI30 s). Showing group means for VI240 s (white circles) and VI30 s (black circles).. 
slope, is said to be more resistant to change. On most of the figures the differences between the enriched and deprived rats are small but consistent.

Figure 2.4 showed that pre-feeding was generally more disruptive to deprived rats than enriched rats. Figure 2.4 shows that the unfilled circles representing the enriched rats average data, are above that of the deprived rats (filled squares) in all cases, so the enriched rats are maintaining response rates closer to baseline. Also the slope of a straight line fitted to these points (by least squares regression) is shallower for the enriched rats, showing that as prefeeding amounts increased the enriched rats' response rates drop more slowly.

As different amounts of pre-feeding were used with the two groups, they were analysed using separate mixed design ANOVAs. With both groups, response rate declined significantly with the amount pre-fed $(F=35.22 / 50, d f=3,30, p<0.05)$, and with a significant difference between enriched and deprived rats $(F=6.82 / 6.80, d f=1,10, p<0.05)$. For the first group (which was pre-feed relatively smaller amounts) there was also a significant interaction between prefeeding amount and housing condition $(\mathrm{F}=2.94, \mathrm{df}=3,30, \mathrm{p}<0.05)$, but with the second group there was not $(F=0.61, \mathrm{df}=3,30, \mathrm{P}>0.05)$.

In the case of novel objects (Figure 2.5) the number of objects did have an effect on responding (mixed design ANOVA, $F=271.22$, $d f=3,30, p<0.05$ ) but this effect was not systematic. On average, across the three conditions the deprived rats were slightly more disrupted but this difference was not significant $(F=40, d f=1,10, p>0.05)$, nor was there a significant interaction $(\mathrm{F}=0.61, \mathrm{df}=3,10, \mathrm{P}>0.05)$. 


\section{PRE-FEEDING}

enricher

- depriver
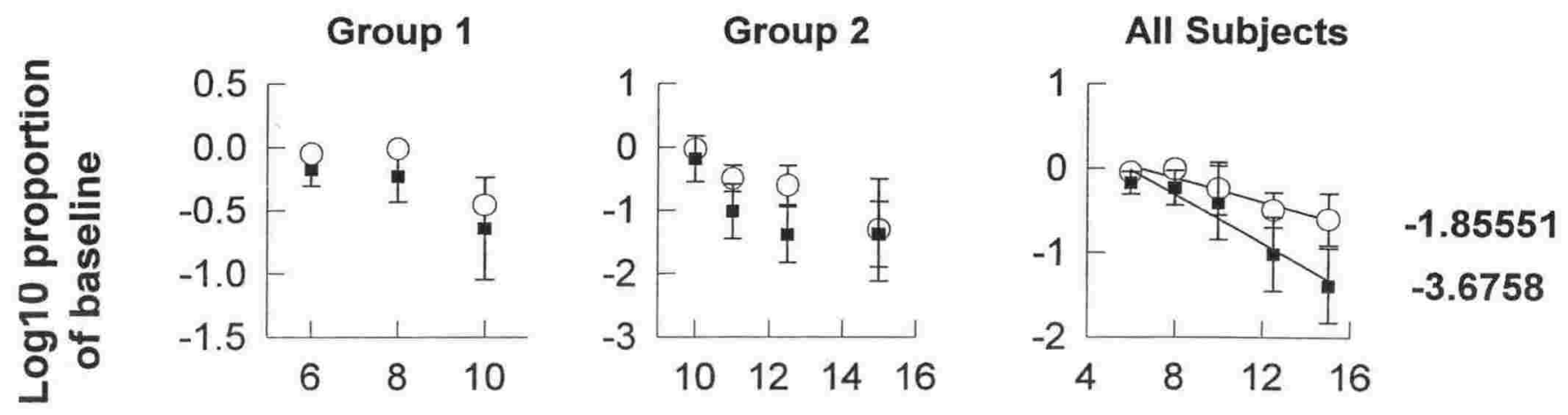

Sweetened condensed milk (m/s)

Figure 2.4 Log proportion of baseline responding maintained under conditions of disruption due to pre-feeding. Data are group means and standard errors. The three columns show the data for group one, group two and a summary of both. White circles are enriched rats, and black squares are deprived rats. (Some of the smaller error bars are obsured by the symbols, on the first figure). 
enriched

- deprived

\section{NOVEL OBJECTS}
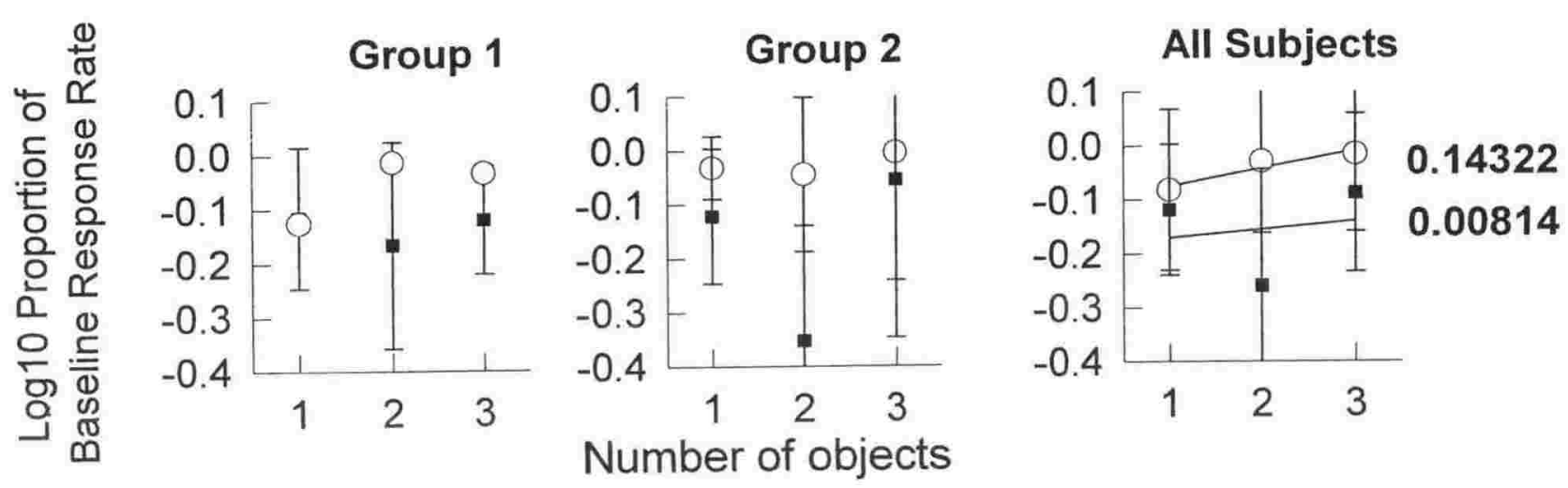

Figure 2.5 Log proportion of baseline responding maintained under conditions of disruption due to novel objects. Data are group means and standard errors. The three columns show the data for group one, group two and a summary of both. White circles are enriched rats, and black squares are deprived rats. 
enriched

- deprived

\section{EXTINCTION}
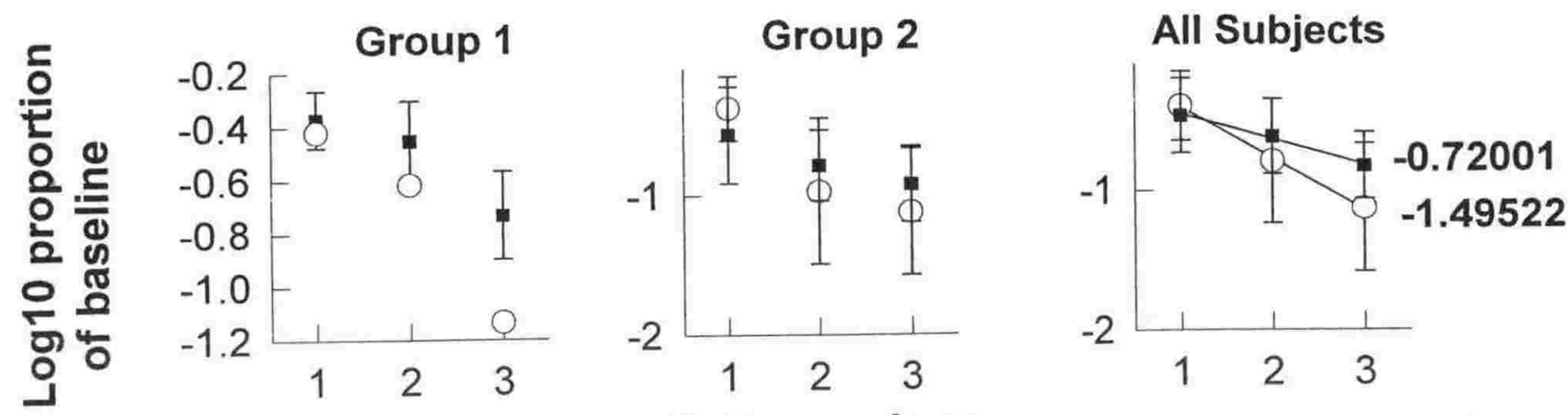

Daily sessions

Figure 2.6 Log proportion of baseline responding maintained under conditions of disruption due to extinction. Data are group means and standard errors. The three columns show the data for group one, group two and a summary of both. White circles are enriched rats, and black squares are deprived rats. 
Figure 2.6 shows that deprived rats are less disrupted by extinction than enriched rats.

A mixed design ANOVA showed main effects for housing group ( $F=9.90$, $\mathrm{df}=1,10, \mathrm{p}<0.05)$ and session $(\mathrm{F}=3.88, \mathrm{df}=3,30, \mathrm{p}<0.05)$, but with no significant interaction $(F=2.6, d f=3,30, P>0.05)$.

\section{Discussion}

This experiment suggested that there is no single effect of housing on resistance to change Those housing effects that are apparent must be treated cautiously as this procedure did not replicate the finding that behaviour on rich schedules is more resistant to change than lean schedules. When a trend is apparent it is the enriched rats who were more resistant to disruption by pre-feeding, while deprived rats were more resistant to disruption by extinction of reinforcement. This is consistent with Davenport et. al. (1975) who found that deprived rats respond more than enriched rats during extinction. However it also shows that extinction may not act in the same manner as the pre-feeding. The novel objects data are questionable, in that disruption did not increase as the number of objects was increased. Overall this experiment's data may be considered suggestive, but they require replication with subjects that clearly demonstrate the effects of schedule richness on resistance to change, prior to the introduction of other variables.

In the absence of consistent effects of environmental conditions on either memory or persistence there was one more cognitive deficit that could explain the Hebb William's maze 
results and possibly clarify the meaning of the current data. It is possible that the deprived rats suffer from a simple learning deficit. If the deprived rats remember their previous responses, and are not more resistant to change, then the lack of change might be due to an inability to learn from these experiences in the first place. 


\section{EXPERIMENT 2.3}

Learning

At least one article has indicated that the Hebb William's maze is a measure of "learning performance" (Shibagaki et. al., 1981). Learning involves linking behaviour with a consequence and the setting where its occurrence reliably produces the consequence. It is not necessary at this point to try and decide exactly which of these two links might be impaired in a deprived rat, so a general test of learning can be used. The task chosen was learning to discriminate between a rich and a lean VI schedule. The task was similar in many ways to the visual discrimination tasks that have been commonly used in the past (Bingham \& Griffiths 1952; Gill et. al., 1966; Krech et. al., 1962; Woods et. al., 1960 etc.).

The visual discrimination task used in previous experiments required rats to chose one of two stimuli, when one was always paired with a reward and one was never paired with a reward. The results were analysed in terms of percent correct which provided a fairly limited amount of information. Multiple VI VI schedules also use two stimuli; typically one that often produces a reward, and one that rarely produces a reward (on VI schedules). This means that the best strategy is to respond frequently to the lever that provides access to a rich reinforcement schedule, and only occasionally on the lever that provides access to the lean schedule. Rats that successfully match their response rates to the rates of reinforcement will obtain the maximum amount of food for the minimum amount of effort. This kind of data can be analysed using a range of more sophisticated and informative analysis techniques including the use of the Matching Law as discussed in Part 2.3.2. 


\section{Part 2.3.1: Visual Discrimination Reversal}

The visual discrimination procedure involved reinforcing animals for responding exclusively to one of two available stimuli. Once they reached a certain level of accuracy (say $80 \%$ correct), the requirement changed so that reinforcement was gained only by responding to the previously unreinforced stimulus (a discrimination reversal). Modifications of this basic procedure were used to assess many aspects of learning performance. With time the visual discrimination procedure has been increasingly replaced by procedures based on variable interval schedules analysed using equations such as the Matching Law (as in Part 2.3.2 to follow). Part 2.3.1, replicated a standard visual discrimination procedure with two exceptions. First, VI30 s and VI240 s were used in the place of continuous reinforcement and non-reinforcement respectively. Second, the schedules were presented consecutively rather than simultaneously to avoid the possibility that rats could respond to a position bias. The replication of the other features of the visual discrimination procedure (the use of reversal etc.) was to allow hypotheses to be made based on the discrimination literature.

This visual discrimination based procedure allowed investigation of the notion that deprived rats are impaired in reversal learning. The previous experiment suggested that this is not due to a greater resistance to change. The differences in reversal learning may be due to a learning deficit, or to a specific confounding variable relating to the use of continuous reinforcement and/or aversive aspects of the testing apparatus. 


\section{Part 2.3.2: The Matching Law}

Part 2.3.2 of the experiment made further use of VI schedules to analyse the learning behaviour of enriched and deprived rats. The procedure was altered to allow the use of an analytical method called the Matching Law (Baum, 1974).

The relative number of the animals' responses that occured in the presence of one of two schedules, is generally proportional to the relative amount of reinforcers available on that schedule (Herrnstein, 1970). The schedules used in this experiment were multiple VI240 s VI30 s, multiple VI53.3 s VI53.3 s and multiple VI30 s VI240 s. Learning rate was addressed rather than the subjects' asymptotic levels of performance which are more reflective of capacity than learning. Pre-asymptotic learning was assessed by allowing the subjects only twenty sessions experience of each schedule combination. Subjects that learn rapidly will reach a higher level of performance within this time period, than slower learners do.

Experiment 2.1 suggested enriched rats sometimes show higher asymptotic levels of performance on a visual discrimination. However, under non-distracting circumstances there were no differences. The intention of this experiment was to study learning under optimal conditions rather than the effect of attentional variables. For this reason an automated experimental chamber was used as it provided the most enclosed and uninterrupted environment. It is also noted that VI schedules result in less abrupt changes in reinforcement rate between condition, than continuous reinforcement would.

The data were analysed by plotting the proportion of responding to one of the two schedules against the proportion of total reinforcers provided by that component, on $\log / \log$ co- 
ordinates. When the data is plotted in this way its can be well described by the Generalised Matching Law

\section{Equation 2.3}

$$
\log _{10}(B 1 / B 2)=a \cdot \log _{10}(R 1 / R 2)+\log _{10} C
$$

Davison and McCarthy (1988)

where $\mathrm{B} 1$ and $\mathrm{B} 2$ are the response rates on the two components and $\mathrm{R} 1$ and $\mathrm{R} 2$ are the reinforcer densities of the two components. $c$ is the elevation of a least squares regression line fitted to the data, and represents response bias. $a$ is the slope of the line and this parameter is used as the measure of the animal's sensitivity to changes in reinforcement, used here as an estimate of extent of learning after a set period of training.

The slope of this Matching line provides a measure of how closely the animal's relative response rate matches the reinforcement rates available across the schedules (as outlined in Baum, 1974; Davison \& McCarthy, 1988). A steeper slope indicates that an animal that is matching its responding more closely to the reinforcement available than an animal whose data produces a more shallow slope.

\section{Method}

\section{$\underline{\text { Subjects }}$}

Part 2.3.1: Visual Discrimination Reversal

The subjects were 12 female Hooded Norway rats (6 enriched and 6 deprived using the housing procedure from Experiment 1). 


\section{Part 2.3.2: The Matching Law}

The subjects had previously served in Experiment 1, however some subjects from Experiment 1 were not used here because they had subsequently been rehoused under other environmental conditions. The groups sizes for this condition were; enriched 8, semi-enriched 8 , standard 4 and deprived 7.

\section{Apparatus}

Two experimental chambers (as described in Experiment 2.1).

\section{Procedure}

\section{Part 2.3.1: Visual Discrimination Reversal}

The rats were auto-shaped to respond on a lever to obtain sweetened condensed milk. They then operated on a VI30 s schedule and the lever light was lit for one minute periods, alternating with dark one minute periods. Subsequently VI30 s and VI240 s schedules were paired with the one minute components. For half of the subjects the VI30 s was originally paired with the lit component, and for the other half the VI240 s was paired with the lit component (this initial learning period is called Condition A). After all subjects showed stable response proportions on each schedule, Condition B began. The stimulus that signalled the VI30 s schedule now signalled the VI240 s and vice versa. After a further 30 sessions this reversal was repeated (Condition C), and 30 further sessions were run. 


\section{Part 2.3.2: The Matching Law}

This experiment followed the same procedure as Part 2.3.1 with the following changes. All of the conditions lasted for 20 sessions, and the conditions were VI240 s/VI30 s, VI30 s/VI240 s and VI53.3 s/VI $53.3 \mathrm{~s}$.

\section{Results}

The data used to construct the figures in this experiment are shown in Appendix 2.3.

\section{Part 2.3.1: Visual Discrimination Reversal}

The rats performance on each component was assessed by averaging the individuals' data over five day periods. The proportion of responses to component S1 was calculated as the total responses to $\mathrm{S} 1$ divided by the total responses during the session.

For half of the subjects S1 was the lit component, and for the other half it was the unlit component. Prior to learning anything about the components the rats would respond equally to both components, producing a ratio of 0.5 . A VI30 s schedule provides up to eight times the reinforcers that are provided by VI240 s schedule. If the rats responded optimally it would respond eight times more frequently to the component signalling the VI30 s schedule.

On Figure 2.7 the black horizontal line shows chance levels of performance. The dotted line shows optimal performance, a ratio of $1 / 9$ for conditions $A$ and $C$, and a ratio of $9 / 1$ for condition B. A learning advantage would produce data that moved rapidly from a response 
A B $\quad$ C $~-0$ - enricher

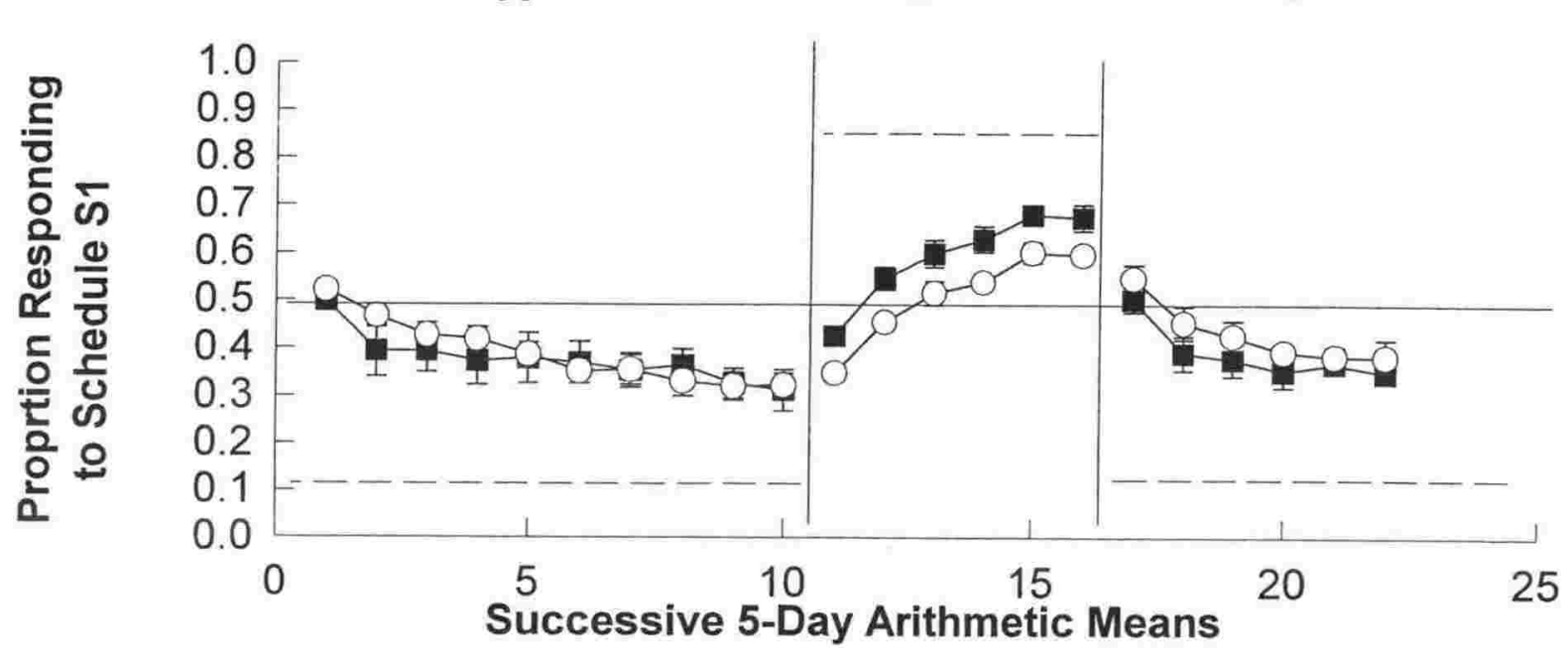

Figure 2.7 Mean proportion responses to S1 (and standard errors) by enriched and deprived rats, as a function of successive five day averages. Showing initial learning and two reversal conditions (Conditions A, B and C). White circles indicate the enriched rats and black squares the deprived rats. 
proportion of 0.5 to a response proportion near the dotted line. This figure shows arithmetic means and standard error bars for the enriched and deprived groups.

In all three conditions both enriched and deprived groups demonstrated learning (mixed design ANOVA, $\mathrm{F}=35.29, \mathrm{df}=9,90, \mathrm{p}<0.05$ ). In Condition $\mathrm{A}$ there is no significant effect of housing group on overall performance (mixed design ANOVA, $F=0.31, \mathrm{df}=1,10, \mathrm{p}>0.05$ ), but there was a significant interaction suggesting that the deprived rats learning rate was slightly faster than that of enriched rats (mixed design ANOVA, $F=2.82, \mathrm{df}=9,90, \mathrm{p}<0.05$ ).

On the first reversal (B), once again there was significant learning effect (mixed design ANOVA, $\mathrm{F}=11.13, \mathrm{df}=1,10, \mathrm{p}<0.05)$, but in this case there is a significant effect of housing $(\mathrm{F}=86.89, \mathrm{df}=5,50, \mathrm{p}<0.05)$ and no interaction between learning and housing condition $(\mathrm{F}=0.28, \mathrm{df}=5,50, \mathrm{p}>0.05)$. So deprived rats performed closer to the optimum than enriched rats, but they did so as soon as the reinforcer densities changed, without a period of learning being necessary. This finding held when individual sessions were used rather than five session totals (mixed design ANOVA interaction not significant, $\mathrm{F}=1.17 \mathrm{df}=5,50, \mathrm{p}>0.05$ ).

Finally, on the second reversal $(C)$ only the learning was significant $(F=37.80, d f=5,50$, $\mathrm{p}<0.05)$, and housing did not affect learning rates $(\mathrm{F}=1.23, \mathrm{df}=1,10, \mathrm{p}>0.05)$ nor was there an interaction $(\mathrm{F}=0.66, \mathrm{df}=5,50, \mathrm{p}>0.05)$. Thus, enriched and deprived rats performed equally well during this condition.

In summary, deprived rats showed a minor learning advantage in initial acquisition, and they had an immediate advantage on the reversal that was fully apparent by the end of the first 
reversal session. Subsequently the deprived rats maintained this advantage and seem to reach a higher asymptotic level of performance than enriched rats. However, on the second reversal there were no significant group differences.

\section{Part 2.3.2: The Matching Law}

As in Part 2.3.1 the rats proportion of responding to one of the two components was calculated as an average of the last five days of the condition. The individual's ratio of responses in S1 (versus total response rates) was plotted on $\log / \log$ co-ordinates as a function of the ratio of reinforcers programmed to be available in S1 (versus total reinforcement). A straight line was fitted (by least squares regression) to each individuals' data to obtain estimates of the matching law equation. As discussed in the procedure section, a steeper slope reflects that a rat has learned to match its responding more closely to the reinforcer rate. A shallow slope means that response rates in the presence of each stimulus were the same regardless of which schedule was in force.

Figure 2.8 shows the group mean slopes of the matching lines fitted to the individual data, and the mean matching lines for the four housing groups. The top figure shows that all of the subjects produced data with fairly shallow slopes (0.1 to 0.25$)$. Given time, subjects will typically produce data with slopes of 0.3 to 0.4 , on tasks of this type. So the current data suggests that these subjects were still in the early stages of learning about the reinforcement conditions. However, both figures show that the more enriched groups produced steeper slopes, thus more rapid learning, than the more deprived groups. This effect is not significant as measured by a Kruskal Wallace ANOVA $(\mathrm{H}=8.22, \mathrm{df}=3,35, \mathrm{p}>0.05)$. However it is worth 

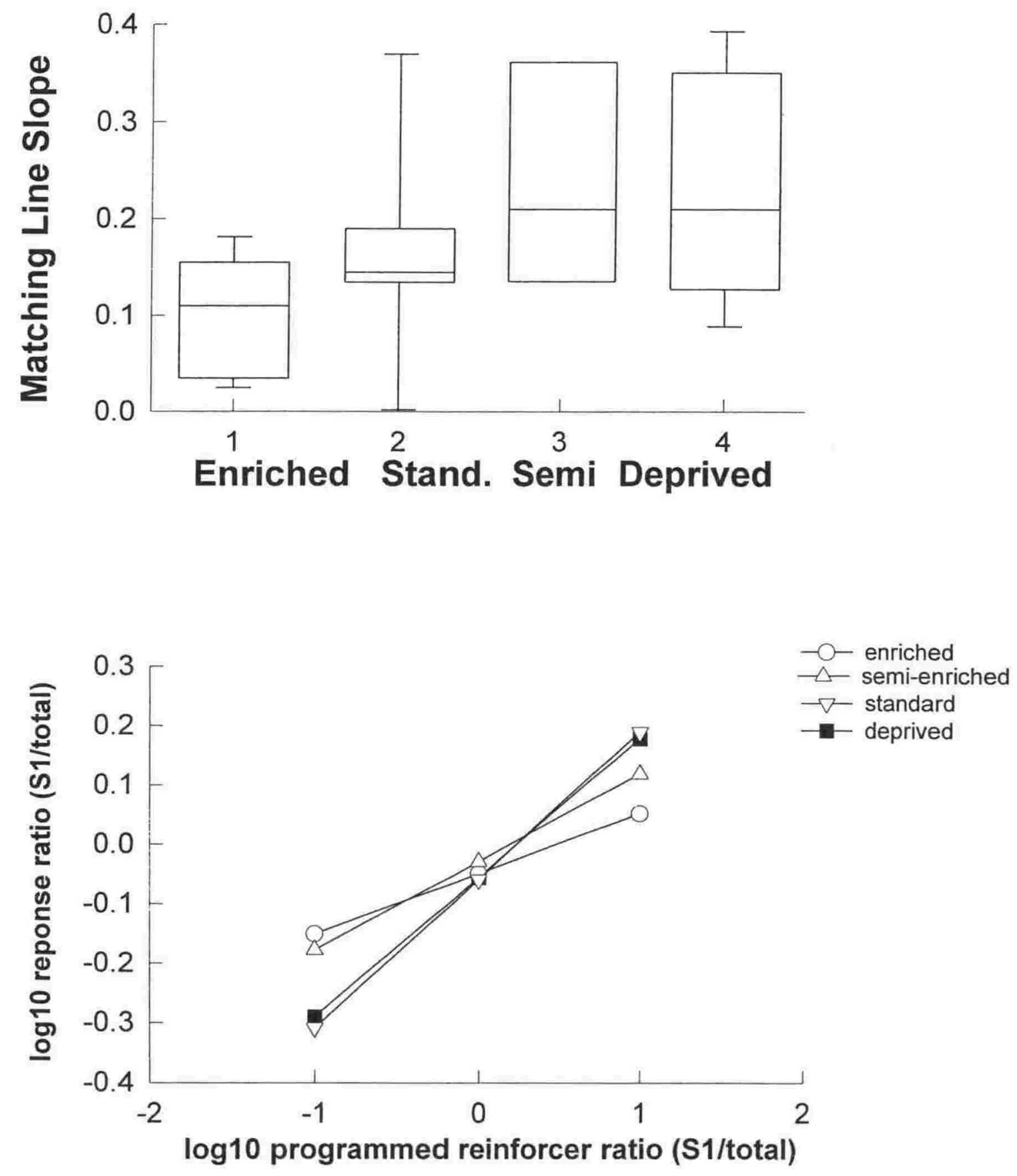

Figure 2.8 Box and whisker plot of the matching line slopes, and mean matching lines for enriched, semi-enriched, standard and deprived groups. 
noting that if the enriched and deprived groups are considered in isolation, there is a significant group difference (Mann-Whitney $\mathrm{U}=-2.11, \mathrm{df}=6,7, \mathrm{p}<0.05$ ).

\section{Discussion}

In Part 2.3.1 the deprived rats showed a minor advantage in initial learning, and their initial response to the first reversal were more optimal than that of the enriched rats in that they more closely matched the scheduled response rates. The reversal result raised the possibility that deprived rats were more sensitive to changes in reinforcement density changes. Such a sensitivity would allow rapid adaptation to change that is not based on a long period of learning, as shown by the deprived rats during Condition B. Sensitivity differences were further implicated by Part 2.3.2, where the deprived rats matched their responses rates more closely than the enriched rats, to the reinforcer densities of the two schedules. This resulted not only in the enriched rats showing no learning advantage, but performing somewhat more poorly than the deprived rats.

There is no evidence in this experiment that the deprived rats learned more slowly than enriched rats. Thus, there is nothing in this experiment to suggest that the deprived rats ability to learn is implicated in their poor Hebb William's maze performance. The results from Part 2.3.1 (visual discrimination) were, however, inconsistent with the existing continuous reinforcement literature, which found that the enriched rats were superior performers under conditions of reversal (Bingham \& Griffiths 1952; Gill et. al., 1966; Woods et. al., 1960). 
So we must first explain why the enriched rats have an advantage in previous discrimination studies but not in the current VI schedule variant. It has been suggested that the enriched rats learning advantage is more obvious on complex tasks than on simple tasks (Renner \& Rosenzweig, 1987). This idea seems to be based on the fact that there is seldom any group difference on visual discrimination learning, sometimes the enriched rats are superior on discrimination reversal, and they are always superior in the Hebb William's maze. This theory does not explain why deprived rats performed well on the VI schedule reversals, so the following is a theory that would explain the existing data and the experiment in Part 2.3.1.

It is possible that there are some problems with the previous visual discrimination procedures. Krech et. al. (1962) tested their subjects using a maze-like structure which may well share the same problems with exploratory confounds as the Hebb William's maze has been criticised for. The other procedures used are water mazes (Woods et. al., 1960) and Lashley jumping stands (Bingham \& Griffiths, 1952; Gill et al., 1966). These procedures could easily have an unforeseen detrimental effect on the performance of the more fearful deprived rats, as one involves water which is very aversive to rats, and the other regularly employs electric shock to facilitate jumping. Even in the absence of aversive stimuli the deprived rats might be disadvantaged by their susceptibility to disruption, as discussed in Experiment 2.1, and shown in the pre-feeding disruption studied in Experiment 2.2.

Greater fear responses or poorer attention might well explain the tendency for deprived rats to perform poorly when they do not in fact suffer from a learning deficit. It does not, however, explain one existing experiment that used an experimental chamber which should not be aversive to the deprived rats (Jones et. al., 1991) and yet they also replicated the finding that 
enriched rats performed better than deprived rats on a discrimination reversal. The major difference between the current experiment and that of Jones et. al. (1991) is that this experiment used intermittent rather than continuous reinforcement and so this is probably responsible for the difference between their result and those in this experiment. Jones et. al. (1991) like all of the previous visual discrimination papers, rewarded every correct response by the rat - rather than using a VI schedule as was done here.

It has been recognised that under conditions of continuous reinforcement (CR), discrimination reversal is very frustrating for animals (Maier, 1949, cited in Gill et. al., 1966). In other words

"If every response has been reinforced, the first omission of reinforcement frequently is followed by signs of disturbance, often identified with "frustration". One of the attributes of this frustration is an increase in the vigor of whatever responses are occurring at the moment." (Kling, 1972, p.576).

A CR discrimination reversal involves moving from continuous reinforcement to extinction on one schedule, and from extinction to continuous reinforcement on the other schedule. It is possible that if one group of animals that was more likely to make these frustration responses (but had no other learning deficit), they would perform poorly on discrimination reversals. However, the frustration prone animal would perform as well as other groups on initial learning of the discrimination, as there is no abrupt extinction process at work during the initial training. There are good reasons to argue that the deprived rats might be more prone to frustration responses than enriched rats. 
There is a range of evidence from studies on emotionality (summarised in Chapter 1) that suggests that deprived rats are more reactive than enriched rats in a number of circumstances. They are more fearful in a novel space, they are more exploratory in a familiar or enclosed space. Experiment 2.2 demonstrated that deprived rats tend to respond more than enriched rats during extinction which could indicate a frustration response. In a discrimination reversal the frustration response would consist of continuing to respond on the previously reinforcing key, and these responses would count as errors and cause the rat to score poorly in a learning task. It would also effect other transfer of learning tasks where the old strategy must be extinguished before the new one is learnt (e.g. Morgan, 1973).

However, extinction frustration would not occur when variable VI were reversed - as neither schedule goes into extinction. The change in reinforcer density will only gradually become apparent, and no discrete error response is made by the animal. In other words, the animal working on a VI schedule has no experience of responding in the presence of a stimulus producing a reward $100 \%$ of the time. Thus it does not experience a sudden disparity between its recent reinforcement history and the current reinforcer contingencies, when the schedules are reversed.

One way to test this would be to analyse visual discrimination accuracy in terms of the accuracy of the previous response. For example, one incorrect response would be likely to lead to a burst of other incorrect responses if frustration was the main reason for the performance deficit. Chamove (1994) found this type of frustration reaction in monkeys with a specific form of brain damage. These monkeys responded poorly on trials immediately following error trials, but well after correct trials or under errorless discrimination procedures. 
The rat's apparently long lasting frustration reaction might take a similar form, reappearing with each unsuccessful response.

So, the deprived rats may show a more marked response to extinction, and this causes their greater errors on discrimination reversals. This is consistent with the earlier finding that deprived rats responded more than enriched rats during extinction (Experiment2.2) even though they were more affected than enriched rats by other forms of disruption.

The 'Frustration' argument could explain why the deprived rats showed no performance deficit on the VI matching task. This argument then provides a basis (of equal group perfromance) for explaining how the deprived rats might show be superior to enriched rats in Experiment 2.3. The explanation may lie with the deprived rats tendency to be more exploratory than enriched rats in familiar or enclosed environments. If the deprived rats heightened exploratory activity extends to operant tasks it might result in them being more sensitive to reinforcement, and thus better at matching on multiple schedules.

Part 2.3.2 also showed that the deprived rats tended to show higher overall response rates, an average of 820 per session for deprived rats, but only 462 for enriched rats. This is consistent with other results that show that deprived rats respond more frequently than enriched rats on DRL schedules (Ought et. al., 1972), contra-freeloading procedures (Coburn \& Tarte, 1976), extinction (Davenport et. al., 1976) and shaping (Rose, 1988). However, it would be more informative to see how these response rates varied according to reinforcement densities of single schedules. This would show whether the higher response rate of the deprived rat occurred at all levels of reinforcement or if it is part of a more variable pattern of behaviour. 
This could be combined with a direct assessment of the effect of housing on reinforcer sensitivity, as shown in Experiment 2.4 


\section{EXPERIMENT 2.4}

The aim of the first three studies in this chapter was to investigate the presence or absence of cognitive differences between the enriched and deprived rats. After investigating the three major aspects of cognitive performance there was no evidence of a severe or global cognitive difference (such as could be described as intellegence or problem solving). The best explanation identified to explain the directions of the small group differences that were present, was that deprived rats may have a greater sensitivity to a range of novel stimuli or situations, including a greater sensitivity to reinforcement. If the deprived rats were more sensitive to reinforcement, any given reinforcement should have a greater effect on their behaviour than it would on the enriched rats.

This idea needs to be tested by measuring the effectiveness of set reinforcer levels on deprived and enriched rats. Herrnstein (1970, for example) has outlined a method for assessing reinforcer efficacy. This involves recording the animals' response rates on a single VI schedule set at various reinforcer densities. An animal that is more sensitive to reinforcement should make more rapid and marked changes in its response rate as the value of the VI schedule changes. This kind of data can be described by a negatively accelerating function.

Equation 2.4

$$
B=\frac{k \cdot R}{R+R o}
$$

(Herrnstein, 1970) 
This function incorporated response rate $(B)$, and reinforcement rate $(R) . K$ is the asymptote of the function and it has been described as a measure of motor capacity (Heyman, 1983, p.114). With enriched and deprived rats it might also bear some relation to overall activity levels, such as have been implicated as a possible confounding variable in many traditional environmental enrichment experiments.

More importantly, the Ro parameter is the reinforcement rate that maintains a level of responding half way between zero and $\mathrm{k}$, and it is said to reflect reinforcer efficacy (Heyman, 1983, p.114). When the reinforcer type is held constant, differences in Ro must reflect the subjective magnitude of the reinforcer, as opposed to its physical amount. Thus if the deprived rats are more sensitive to reinforcement, their data should result in lower Ro values than those of the enriched rats.

\section{Method}

\section{$\underline{\text { Subjects }}$}

The subjects were 5 enriched and 7 deprived female Norway hooded rats which were approximately 60 days old at the beginning of the experiment, the uneven group sizes were due to an error in the distribution across conditons when the subjects were 30 days old (see Experiment 1 for details of the enriched and deprived housing procedures).

\section{Apparatus}

Two experimental chambers (as described in Experiment 2.1) were used. 


\section{$\underline{\text { Procedure }}$}

The rats were auto-shaped to press the lever in the chamber. They then began the experimental sessions which were 45 minutes long, the lever light was on and a single VI schedule was in force throughout the session. In the first condition the schedule was VI240 s, the rats remained on this schedule until they all showed visually stable data (after 37 days). The schedule was then changed every twenty days in the order; VI150 s, VI75 s, VI30, VI10 s and VI5 s.

\section{Results}

Figure 2.9 shows total session response rates as a function of reinforcers per hour, averaged across the rats in each housing condition. The response rates used were arithmetic means from the last five sessions of each condition. The lines fitted to the group data have very similar asymptotic levels suggesting that there is little difference between the enriched and dęprived rats' $K$ values. However the line fitted tonthe utepriveu' rat's uata acceleratea towara's the asymptote more rapidly than that of the enriched rats, suggesting a difference in $R o$ values and therefore in reinforcer efficacy.

Hyperbolas were fitted to the data of each subject and the $K$ and $R o$ values derived are shown in Table 2.4.1. Statistical analysis confirmed that there is no significant group differences in $K$ (Mann-Whitney $\mathrm{U}=11, \mathrm{df}=5,6, \mathrm{p}>0.05$ ), but group differences in Ro were significant (Mann-Whitney $\mathrm{U}=1, \mathrm{df}=5,6, \mathrm{p}<0.05$ ). One of the deprived rats produced extremely outlying data, however its inclusion or exclusion did not affect this conclusion. Thus, Ro was significantly smaller for deprived rats than for enriched rats. 
enriched

- deprived

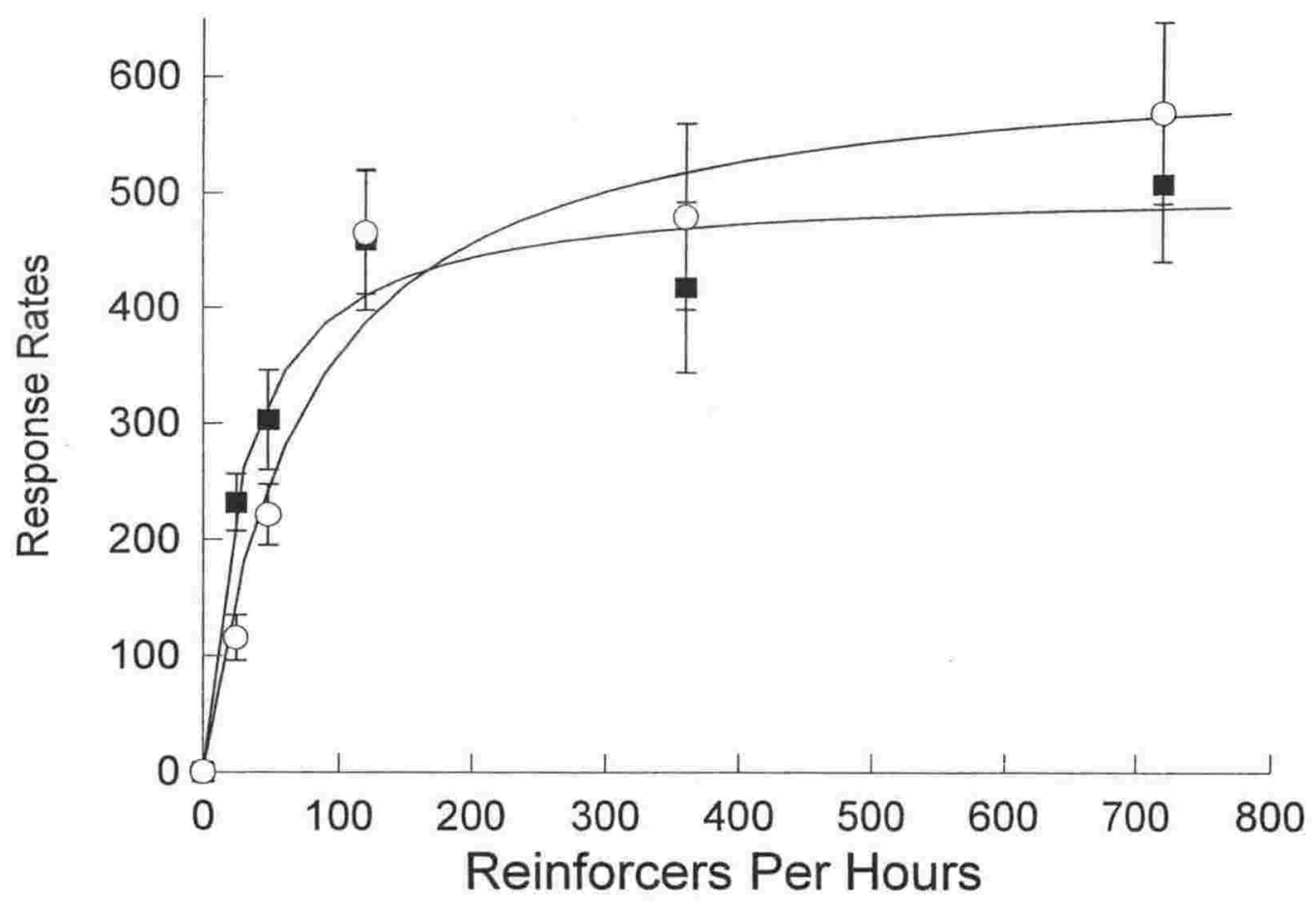

Figure 2.9 Arithmetic means for enriched and deprived rats response rates as a function of. reinforcer density, on a single schedule, with standard error bars. Negatively accelerating curves have been fitted to the mean data using Equation 1. 
Table 2.4.1

$\underline{K \text { and Ro values from Herrnstein's hyperbolas fitted to the individual data of seven deprived }}$ and five enriched rats.

\begin{tabular}{|l|l|l|}
\hline Rat & Deprived $\boldsymbol{K}$ & Deprived $\boldsymbol{R} \boldsymbol{o}$ \\
\hline A & 316.29 & 36.28 \\
\hline B & 519.19 & 22.10 \\
\hline C & 25.29 & -327.10 \\
\hline D & 673.03 & 43.60 \\
\hline E & 545.08 & 24.69 \\
\hline F & 834.27 & 47.28 \\
\hline G & 399.68 & 61.86 \\
\hline mean & 501.26 & 39.30 \\
\hline & Enriched $\boldsymbol{K}$ & Enriched $\boldsymbol{R o}$ \\
\hline H & 753.26 & 75.84 \\
\hline I & 459.24 & 51.98 \\
\hline J & 838.66 & 111.98 \\
\hline K & 442.27 & 63.24 \\
\hline L & 670.12 & 70.22 \\
\hline mean & 632.7 & 74.65 \\
\hline & & \\
\hline
\end{tabular}

\section{Discussion}

The results fell in the expected direction with the deprived rats being significantly more affected by reinforcement than the enriched rats, but with no clear difference in locomotor capabilities. This provides further support for the idea that the deprived rats heightened sensitivity to aversive situations or novel stimuli, also extends to sensitivity to reinforcement. Combined with the other studies n Chapter 2 it suggests that the perceived cognitive deficit may not only be absent, but under some circumstances the deprived rats might have a learning advantage. 
These results have a number of implications for animal behaviour researchers. They suggest one way in which housing conditions are a relevant variable in behavioural research. However, it would be inappropriate if these results were taken to mean that deprived conditions were preferable for the housing of laboratory rats. It is possible to suggest that the extra sensitivity is due to the animal being deprived of stimulation in its home cage, and so it is attempting to compensate by maximising the effect of stimulation available during the operant session. However, given that cognitive deficits are described as indicators of poor welfare in Experiment 1, one should be careful about explaining this reversal away too easily. It is probably more important to balance the possibility of a small learning advantage with the deprived rats, against the well known enhanced fearfulness that they experience. It is possible that a rat in deprived housing, that has its fearfulness reduced by human handling, might get all the extra stimulation it requires from its experimental sessions. However, rather more information would be required before this kind of housing practise could be justified.

\section{General Discussion}

The major conclusion to be drawn from the Chapter 2 experiments is that the data does not convincingly support the proposition that deprived rats suffer from a significant, global, cognitive deficit. In fact, the results from Experiments 2.1-2.4 suggest that deprived rats can out-perform enriched rats on some operant tasks. Many of the group differences that exist in the data were small, but they were consistent with the deprived rats being more sensitive than enriched rats to a range of stimuli and consequences. The final experiment directly assessed sensitivity to reinforcement experiment, and appears to confirm that this is the case. 
Based on this chapter, it is a strong possibility that the Hebb William's maze is an invalid test of cognitive abilities in a wide range of situations, if not universally. The Hebb-William's maze is intended to measure a broad cognitive ability comparable with intelligence. This does not seem to be the case with environmental enrichment, as no cognitive deficit is apparent using procedures other than the maze. This indicates that the Hebb William's maze should no longer be used to assess the cognitive effects of enrichment. It also means that we need to examine whether this erroneous belief about the cognitive effect of deprivation has formed the basis of any other misconceptions. Holson (1986) wrote ...

"...it is the work with isolated and enriched rats in mazes which provides the most compelling evidence for a strong relationship between early experimental deprivation and later cognitive or intellectual deficits."

This chapter has not demonstrated any such 'strong relationship' between enrichment and cognitive abilities. There appears to be differences in the nature of reactions to disruption and sensitivity to reinforcement. Persistence may be implicated in many of these housing effects on behaviour in Experiment 2.1, but this was hard to reliably produce in Experiment 2.2. One of the disrupters (objects) reduced behaviour without having the usual effect across schedules, one disrupter (extinction) seemed to measure frustration rather than persistence. Thus, only the pre-feeding results offer direct evidence that the deprived rats were less persistent than the enriched rats. So, at best the Hebb William's maze might reflect that the deprived rats perform less persistently on the task, probably because they are disrupted by irrelevant stimuli in the large and complex maze environment (as compared with their home cages). It does not seem to reflect a general cognitive ability that could be described as intelligence or problemsolving. 
One reason for the relatively modest impact that caging environment seems to have on rats' behaviours, may be due to the proportion of the rats' behaviour patterns that are genetically inherited. A behavioural repertoire made up largely of such fixed action patterns must be more robust against the modifying effects of the environment than those that need to be learnt from the environment. Boice (1977) demonstrated that inbred laboratory rats behave identically to wild rats when both are kept in large outdoor enclosure, he also reports information that suggests that the laboratory rat can become successfully feral. This resistance to the cognitive effect of restrictive housing is not necessarily present in less precocious (able to be independent at a young age)animal species.

Chapters 1 and 2 suggest that caging did have some systematic effects on the behaviour of the rats. The deprived rats were some what more fearful than enriched rats, although other research suggests that this effect can be ameliorated using handling or other nonenvironmental methods (Spence \& Maher, 1962 for example). The deprived cage does not seem to produce a large or consistent cognitive deficit in the rats housed in it. One difference (disruption) suggested the deprived rats had a deficit, the other (sensitivity to reinforcement) suggested the enriched rats had a deficit. So, it may be possible to use deprived caging without significantly reducing the rats welfare with respect to cognitive function.

The lack of large scale and permanent deficits in deprived rats does not necessarily mean that the rat's welfare could not be improved by environmental enrichment. Looking for deficits is rather like putting an ambulance at the bottom of the cliff. It is a procedure that is aimed at detecting fairly significant wide scale damage, and it might not be sensitive to detect the possibility of more modest improvement. Chapter 3 signals a move from a conservative 
damage control approach to a more pro-active search for an advantage that could be gained by enriching the standard cage. 


\section{CHAPTER 3:}

\section{Preference}

This chapter begins by reviewing published studies which have used preference measures to examine the effects of various aspects of the housing environment on the welfare of laboratory rats (Section 3.1). However, because the available data were very sparse, the review was supplemented with data relating to the rat's physical health (Section 3.2). This is followed by a discussion on the theory behind the use of preference procedures (Section 3.3) and the different types of procedure available (Section 3.4).

\section{1) Rats and Their Cages: Preference}

A review of the literature shows that, outside of the traditional environmental enrichment field, there is surprisingly little data relating to the caging of rats. The most commonly used behavioural assessments of caging, have been measures of preference, and even these amount to a relatively small number of studies. This summary aims to bring together the data that does exist, make practical suggestions about how to house laboratory rats, and determine what further information is required.

The most common method of testing preference consisted of placing an enrichment in one half of the normal cage. If the rat spent most of its time on the enriched side then the intervention was considered successful. Sometimes this measure has been supplemented with some observations about the relative frequency of common 
behaviours shown on each side of the cage. The most commonly used objects were barriers, tunnels, nest boxes and nesting materials.

The most extensive study of rats' preferences for cage modifications was by Bradshaw and Poling (1991). They found that rats showed preferences for wooden platforms, wood chips and paper towels over an empty cage. Other studies have found that rats prefer vertical cage barriers (Anzaldo et. al., 1994), cages hung with chains (Denny, 1975) and nest-boxes (Townsend, 1997). No significant preferences were shown for tunnels (Bradshaw \& Poling, 1991), or a pattern of horizontal and vertical barriers (Anzaldo et. al., 1994). Bradshaw and Poling (1991) used direct comparisons to show that rats preferred paper towels over platforms, but it is otherwise difficult to tell which enrichment option would be most preferred.

There are some suggestions about what behaviours the rats show in the environments they prefer. For example, Townsend (1997) measured the impact that the nest box has on the rat's behaviours, but the significance of these effects is not clear. Some behaviours declined in frequency (rearing, walking, eating), some were the same (resting) and some became possible (nest box related behaviours). Although the presence of wood chips results in nesting and chewing (Schneider, 1988), it is difficult to say whether these behavioural changes are necessarily beneficial. However, behavioural observations do help check whether the enrichment is used in the way that its name implies (nest-box, tunnel etc.). 


\section{2) Rats and their Cages: Physical Health}

There is a lack of data on rats preferences for different cage materials, increases in cage size and groups size. In the absence of preference data about space and group size requirements, the following physical data is provided to (at least partially) balance the picture. This is done in the knowledge that physical data will relate onlt loosely to behavioral data, and that health and injury measures are often not particularly sensitive indices of welfare.

Lawlor (1989) summarised the data available on what amount of space is necessary to avoid harm to the rat (such as foot lesions and premature death). She concluded that the largest rats (over $900 \mathrm{~g}$ ) required a cage with at least $1800 \mathrm{~cm}^{2}$ of floor space and $30 \mathrm{~cm}$ in height with each rat in the group having at least $1000 \mathrm{~cm}^{2}$ of floor area. However smaller animals required somewhat less than this. Beyond such dimensions, however, it is debatable whether further increases in space are desirable. Rats reproduce and grow well in cages of modest dimensions (Bantin \& Sanders, 1989), and isolated rats suffer more stress in larger cages than in small ones (Syme \& Hughes, 1972, cited in Bantin \& Sanders 1989). Thus, rats seem to have a limited need for open space.

Many studies have found that rats do have preferences for complex space. For example, rats prefer long narrow cages over square ones of equal volume (Weiss et. al., 1982, cited in Lawlor 1989). Rats prefer a small space broken by barriers over a larger open space (Anzaldo et. al., 1994), and they perform better when the cage incorporates a separate niche for feeding (Nicolaidis et. al., 1979, cited in Bantin \& 
Sanders, 1989). It is possible that rats would benefit from increases in complex, rather than open, cage space.

In terms of group size, rats grow and survive the best when housed in small stable groups of around three (Brown et. al., 1968) to five (Syme \& Hughes, 1972, cited in Bantin \& Sanders, 1989). Lawlor's (1989) summary concluded that “...in rats group size generally has more effect on well-being than cage size". However not all studies find groups size to be a very influential factor (Mundy \& Porter, 1969).

Finally, solid floors are considered better for rats than mesh floors (Mundy \& Porter, 1969). Rats raised on mesh floors have lower body weights and higher adrenal weights. This suggest a failure to grow normally and a state of long term adrenal activation (stress).

\section{Applied to Current Caging}

The current conditions at the V.U.W. animal behaviour laboratory (as described in Experiment 1) provided the recommended rectangular cage, solid floor and woodchips. However, the rats had no nest-box, platforms or paper. The cage dimensions were only $900 \mathrm{~cm}^{2} \times 23 \mathrm{~cm}$. This would be insufficient to keep the largest male $(450 \mathrm{~g})$ or female (355g) according to Lawlor's (1989) guideline. The New Zealand standard group size (of two) is below the recommended 3-5.

The conditions in this laboratory were typical of laboratories throughout New Zealand and the indication that the space and group sizes were not sufficient was worrying. 
However the above recommendations were based on the results from a limited number of studies. There is a strong need for replication. Firstly this would provide more evidence about the reliability (or otherwise) of the findings. Secondly, the space and group size recommendations should be repeated using a preference procedure so that the findings could be compared directly with the data relating to cage objects and fixtures. Thirdly, the preference studies reported all use Sprague-Dawley strain male rats, so a replication is needed with this laboratory's Hooded Norway rats and including female subjects. Finally, the research needs to be extended to a wider range of interventions. Preference can only be used to choose between the options provided and so far only a few possibilities have been examined.

\subsection{Preference Theory}

Tests of an animal's preference provide quick and simple measures that are often relevant to animal welfare. Most researchers extend the meaning of preference data by saying that animals tend to choose in their best interests (as originally outlined by Dawkins, 1976). This means that the most preferred option would be equivalent to the environment least detrimental to its welfare. However, there is debate on whether providing animals with their preferences will always result in an improvement in their welfare.

Researchers have tended to believe that preference tests normally relate to welfare (i.e. Dawkins), that they can relate to welfare if used very carefully (i.e. Duncan, 1978), or that preference does not legitimately relate to general welfare (i.e. Appleby, 1997). These three positions are based on whether the researchers think that the exceptions to 
the rule (that animals choose in their best interests) are insignificant, common, or too prevalent for the rule to hold.

Some examples of concerns about the validity of preference data are as follows. One, some researchers argue that through controlled breeding, domestic animals may have lost the ability to choose in their best interests (e.g. Duncan, 1978). Dawkins (1977) does not accept this possibility. She suggests that animals may be fooled by artificial choices that would not be found in nature, but that domestic animals perform as well as wild ones. Two, the relative novelty of an option will affect preference.

Sometimes animals will show a bias towards familiar environments (Zajonc, 1972; Cowan, 1977), and sometimes towards exploring unfamiliar environments regardless of their quality (Sherwin \& Nicol, 1993). Three, preference tests can only sample a limited number of environments, and a limited number of choices between these environments. Also, the animals choices are affected by a wide range of different variables at different times which affect their choices (Hutson, 1984). For example, the behaviours an animal is prepared to perform vary daily and seasonally, as well with the time that has passed since they were last able to perform that behaviour. These concerns suggest that any generalisation from preference data must be made cautiously, taking into account the amount and diversity of supporting evidence and the animals previous history with the options and the testing apparatus.

Another way to look at preference is that it is a choice between two consequences. As the behaviour that leads to each consequence is equivalent, the consequence chosen would be the one that is most reinforcing. Likewise, when the animal's behaviour is 
unconstrained, the behaviour it performs most often is that which is most reinforcing. Thus the environment that is chosen or inhabited significantly more often, is significantly more rewarding (or less punishing). This reasoning is based on Premack's Principle that...

"More probable behaviors will reinforce less probable behaviors" (Premack, 1959, cited in Mazur, 1998).

According to Premack, 'more probable' behaviours are those that the subject spends more of its time performing. Thus, by observing which environment the animal dwells in most frequently, given either continuous access or repeated choices, we can conclude which of the available options is most reinforcing.

It still remains for the human caretaker to determine whether the preferred option is unacceptably detrimental to the animal's long term health and well-being. It would be rather too much to expect that the raw data make this kind of decision for us. The expectation that it might, may be based on the idea that 'welfare' is a unitary phenomenon with an absolute optimum. Welfare might be, more realistically, thought of as some balance of the conflicting demands of health, freedom and long and short term enjoyment. Given that animals' choices are normally impulsive (Rachlin \& Green, 1972), preference probably relates most closely to the animal's short term enjoyment.

\section{4) Preference Methodology}

There are two main types of preference test, continuous access and discrete choice Tmaze procedures. Animals may be given continuous access to two or more 
environments, and the time spent in each recorded or sampled. The advantage of this method is that the animals choice can be monitored throughout any activity cycles that may affect its preferences. Also a large number of subjects can be monitored at the same time, and more than two options can be provided at the same time.

Exploration is an important variable in a continuous access preferences test. Blom et. al. (1993) claimed that an animal must be exploratory for continuous access sampling to be effective. The subjects must be exploratory so that they seek out and experience all of the options, and are in a position to choose between them. On the other hand, a continuous access test may not be particularly sensitive with highly exploratory animals and/or small environments, because the subjects would often wander into the less preferred environment. Hughes and Black (1973) mentioned that pre-laying pacing in hens would reduce test sensitivity in this way. So the size of the testing environment needs to be made with reference to the levels of exploratory behaviour shown by the animals.

\section{Discrete Choice in a T-Maze}

The other method of testing is to provide the animal with discrete choices (e.g. via a T-maze based procedure) that are followed by a period of time spent in the chosen environment. When provided with only one option the subjects approach latencies may give a measure of welfare (Dawkins 1976), and with two options the choice made indicates preference (Dawkins 1977, 1978, 1982; Hughes, 1975). In this situation entering a particular environment produces a significant and unavoidable consequence, and this might increase the sensitivity of the method. On the other hand, 
the tendency for rats to alternate the direction of T-maze choices would reduce sensitivity. It is not clear how sensitive the T-maze could be expected to be, compared, for example, with the continuous access box.

Two studies have used both continuous access and discrete choice methods in otherwise similar situations. Hughes (1976) and Dawkins (1977) found these two methods provided different results regarding hens floor and space preferences respectively. In both studies a continuous access box detected no difference but the Tmaze did, but the reasons for this difference were unclear. Since this time the issue appears to have been dropped, with most subsequent experiments using only the continuous access procedure; a curious choice given that it appeared to be the less sensitive method in that it failed to detect any significant preferences in situations when the T-maze did so (Hughes, 1976; Dawkins, 1977).

Before embarking on a long experimental programme a shorter pilot experiment was carried out to determine which apparatus and procedures should be used. This involved starting with a single cage modification (novel objects), and comparing the relative performance of the different preference assessment procedures. 


\section{EXPERIMENT 3.1:}

Three Studies on Rats' Preferences for Objects

This experiment compared the consistency and sensitivity of T-maze and continuous access procedures for assessing preference. This experiment also compared two sizes of continuous access box to see whether this, combined with the activity levels of the rats, might affect the sensitivity of the apparatus. The experiment is made up of a number of small pilot studies examing the preferences of rats for small manipulible objects.

Small objects often made up part of an enriched environment for rats (Luchins \& Forgus, 1955; Krech et. al., 1960; Rose et. al., 1986; and others). Renner and Rosenzweig (1960) suggested that the presence of inanimate objects may be a major factor contributing to the enrichment effect. They write "almost any type of object can serve the purpose of enriching the stimulus complexity of the cage environment" .

The presence of objects allows rats to engage in exploratory behaviour commonly called 'investigation'. Investigation involves nose and mouth contact; sniffing, gnawing and licking (Barnett, 1963). Past studies have shown that rats readily engage in investigatory behaviour when objects are introduced into their environment (McCall, Lester \& Dolan, 1969; Morgan, 1973; Einon \& Morgan, 1976; Renner \& Rosenzweig, 1986; Renner, 1987; Widman \& Rosellini, 1989). Investigation is seen as beneficial for the rats as it is seen as a form of learning (Renner \& Rosenzweig, 1986; Renner \& Seltzer, 1991; 1994). 
However, there are now a number of studies that show that contact with such objects is not sustained (Einon \& Morgan, 1967), and that in some situations rats will even avoid novel objects (Cowan, 1977). So on one hand there is the range of active behaviours that rats do show, and can only show, when objects are present. On the other hand there is evidence that this effect might be very short lived and depend on the objects novelty. Only one study has examined rats' preferences for novel objects, rather than simply their use of them. McCall et. al. (1960) found that rats spent more time on the side of an open field that contained objects. However a large novel arena is a very different situation to a home cage, and so this finding may not indicate that such objects would make good home cage enrichment items.

The following experiment is composed of three sections. Part 3.1.1, measured the levels of investigatory behaviour the rats showed towards objects both when they were novel and when they were familiar. Part 3.1.2, examined whether rats choose to be on the same side of the cage as the objects also when they are novel versus when they are familiar. Part 3.1.3, examined whether the estimate of preference was affected by the procedure used. 


\section{Method}

\section{$\underline{\text { Subjects }}$}

Eight female Hooded Norway rats from the Victoria University of Wellington breeding colony were used in Parts 3.1.1 and 3.1.2. They had previously served as subjects in undergraduate laboratories where they responded on simple operant schedules and ran in a T-maze. They were approximately 6 months old at the beginning of the experiment. Part 3.1.3 used six naive female Hooded Norway rats that were approximately 7.5 months old at the start of the experiment. All of the subjects used in this experiment were housed under standard conditions (outlined in Experiment 1) when not in the an experimental apparatus.

\section{Apparatus}

Observations were made using a JVC compact video camera attached to a video cassette recorder of the same make, and a ITC black and white monitor.

The objects used were 36 junk items made from a range of materials (examples were a broken pen, tennis ball, leggo block etc.). All items took up less than one eighth of the space in a standard cage per item, and were light enough for a single rat to move them across the cage floor.

Parts 3.1.1 and 3.1.2 were conducted using standard cages. Part 3.1.2 also used a standard cage bisected by a wooden barrier that was fifteen $\mathrm{cm}$ high and fixed to the cage lid with bolts. Part 3.1.3 used preference cages constructed from standard rat 
cages. All three cage types are shown in Figure 3.1. Cage A consisted of one cage that was bisected by a fifteen centimetre wood barrier. Cage B consisted of two standard cages with the wire tops joined together along the long side. Cage $\mathrm{C}$ consisted of two cages connected by a wooden T-maze with three sliding doors.

\section{Procedure}

During Part 3.1.1, pairs of rats were observed for five minute periods in their home cages for five days per condition (9.30 am and 5.30pm every day). Food and water were available continuously on one side of the cage. Objects were placed in the equipment immediately prior to the first morning session. The conditions related to how many objects were provided and how often they were changed; two changed daily, two not changed, four changed daily and four not changed. Sessions were video taped and coded for investigatory behaviour. This was identified as nose, paw or whisker contact with an object which was coded as present or absent in 30 second point samples. This produced a score out of six for each session, and a total score of 30 after five sessions.

In Part 3.1.2, type A preference cages were used instead of standard cages. The rats position on the objects, or empty side was point sampled every 30 seconds, producing a score out of six for each session and 30 after completing the five sessions in a condition. The objects were on the same side of the cage as the food and water dispenser for every second session. 
a)

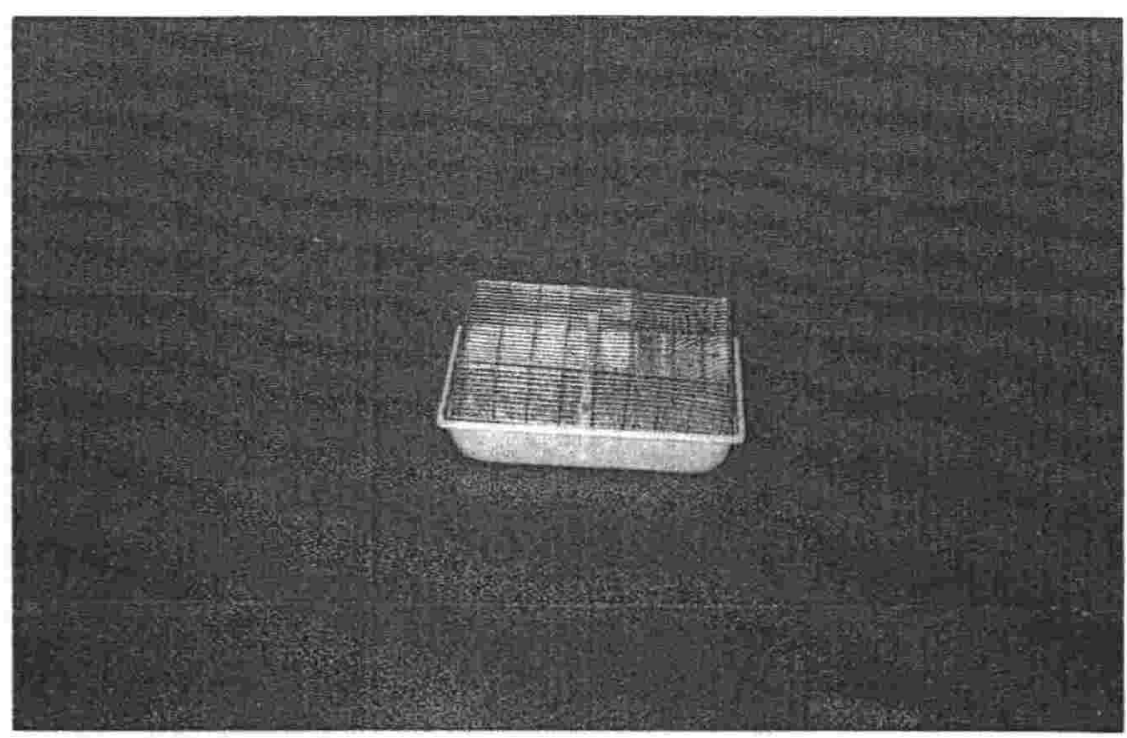

b)

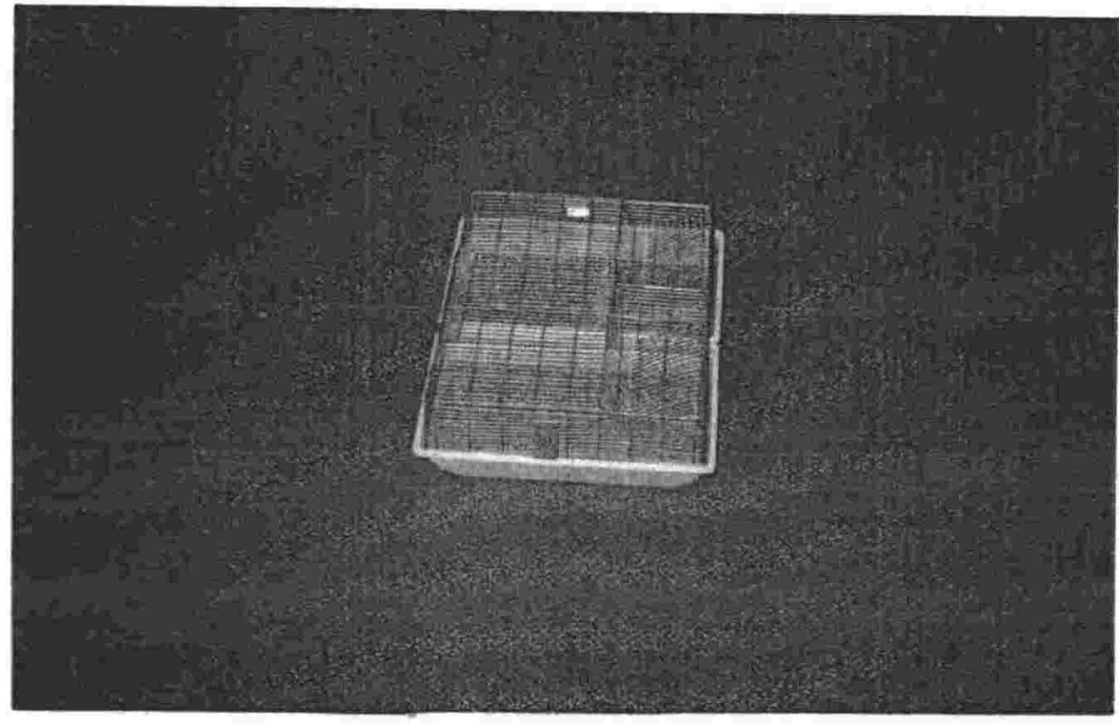

c)

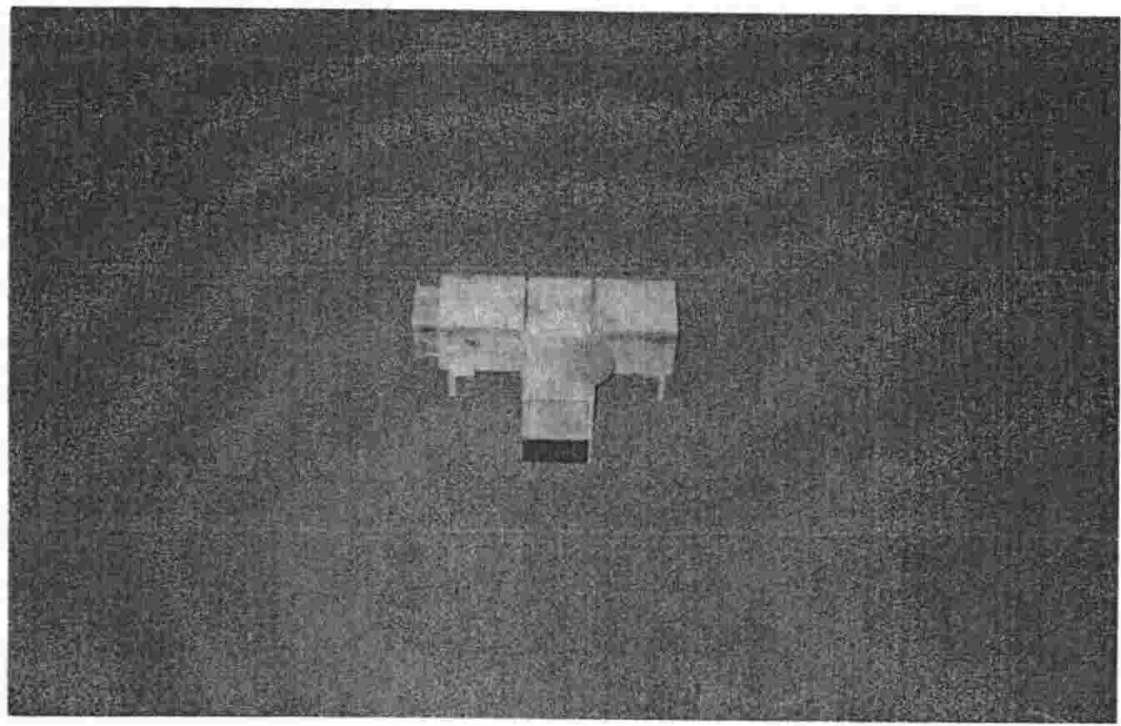

Figure 3.1 a) the small continuous access box, b) the large continuous access box and

c) the T-maze. 
In Part 3.1.3 the rats spent most of the day in home cages and were put into the testing equipment only during their the session, food and water was freely available in the home cage but unavailable during the session. Sessions occurred twice daily at 9.30am and $5.30 \mathrm{pm}$ and lasted for fifty minutes. Two preference options were tested. One, novel objects and wood shavings versus just wood shavings. Two, wood shavings versus no wood shavings. Each rat was tested in each type of cage. Order of testing was counter-balanced for cage type and wood-shavings versus objects.

In the T-maze, a rat was coded as choosing a particular option when all four feet entered the cage. The rat would then be shut in this cage for five minutes during which time the experimenter would leave the room. In each condition each rat completed five trials per session for ten days (total $=50$ ), with the side on which the intervention was presented alternated every session. With the continuous access boxes (Cages A \& B), the rat was placed in the left side of the equipment and its position was time sampled using a remote camera every five minutes. This produced a score out of a total of 50 point samples per subject.

\section{Results}

\section{Part 3.1.1: Investigatory Contact}

Figure 3.2 represents the samples during the sessions when the rats were in investigatory contact with an object. The data shown are means of each subject's score out of 50 . Novel objects were added prior to all of the novel object condition 
morning sessions, and the first morning session of the familiar object conditions. The figure shows clearly that contact was high in morning sessions when new objects were introduced, but dropped to a significantly lower level for sessions when the objects were familiar. When sessions 2 to 5 were compared the investigatory contact was significantly higher during morning sessions in the novel object conditions, than with the familiar object conditions (2-tailed Wilcoxon signed ranks test, $\mathrm{df}=7, \mathrm{~T}=1$, $\mathrm{p}<0.05)$. Contact with objects was low during all of the afternoon sessions, but significantly higher if there were four rather than two objects (2-tailed Wilcoxon signed ranks test, $\mathrm{df}=7, \mathrm{~T}=0, \mathrm{p}<0.05)$.

There was no consistent effect of object number on contact during the morning samples or between changed or unchanged objects during the afternoon sessions (2tailed Wilcoxon signed ranks test, $d f=7, T=1, p>0.05$ ).

\section{Part 3.1.2: Cage Side Occupation}

When the cage was divided to allow the rats to either contact, or avoid the objects, the rats showed no preference one way or the other. This is shown in Figure 3.3 where the rats spend about half of the sample periods in either side (approximately 15/30).

\section{Part 3.1.3: T-Maze of Continuous Access Boxes}

Table 3.1 shows preference scores out of 50 for objects and sawdust, as measured by the three apparatus (the T-maze, large continuous access box and small continuous access box). Based on a binomial probability distribution, a subject making random choices would, on average, produce a score of approximately 25 , whilst a score of 28 


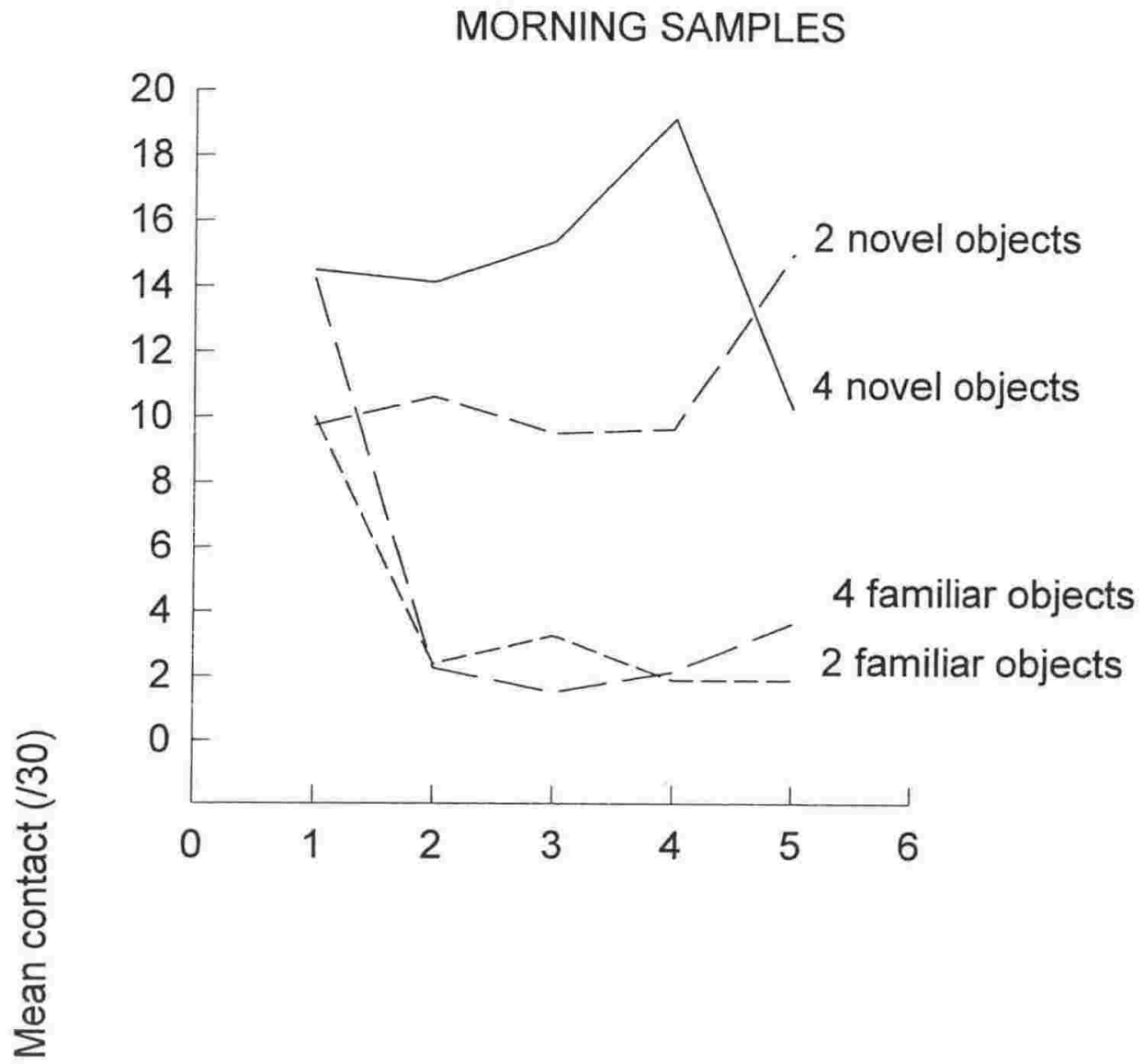

AFTERNOON SAMPLES

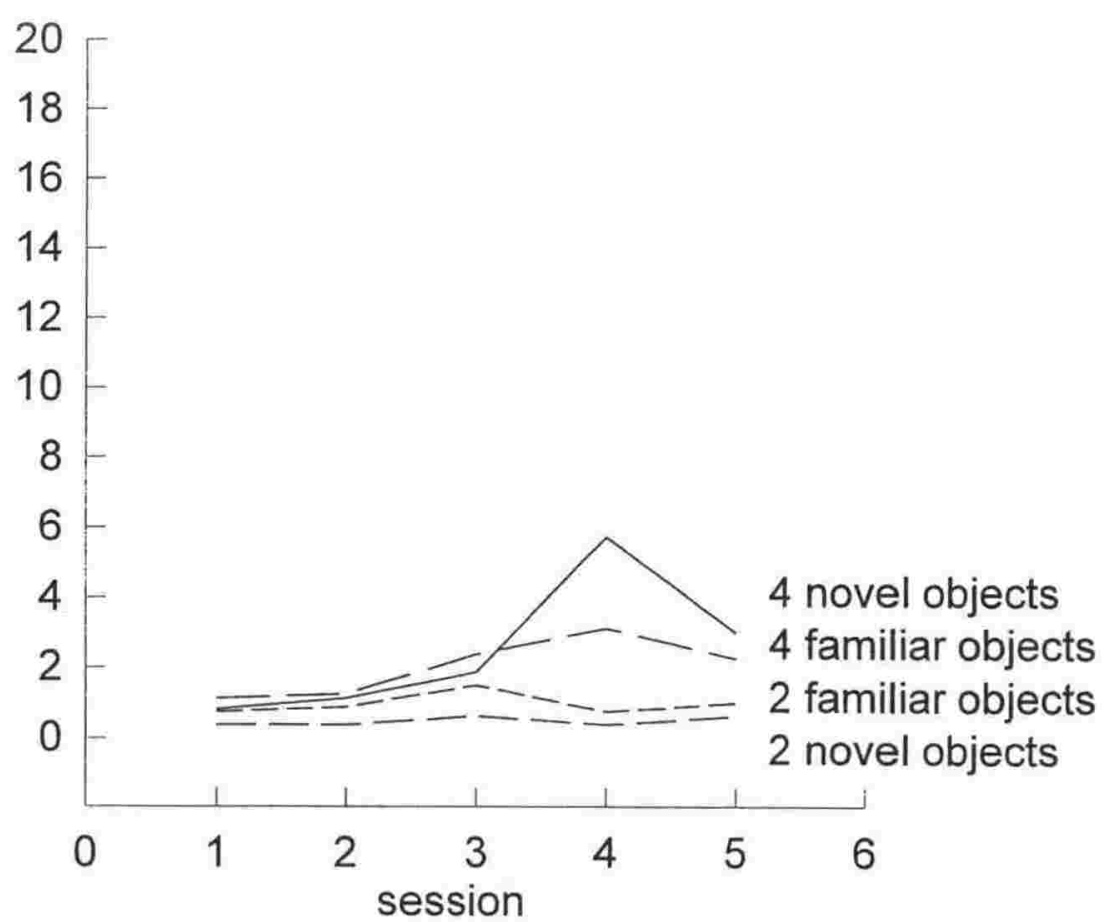

Figure 3.2 Mean contact with objects (/30) as a function of consecutive morning or afternoon point samples. 


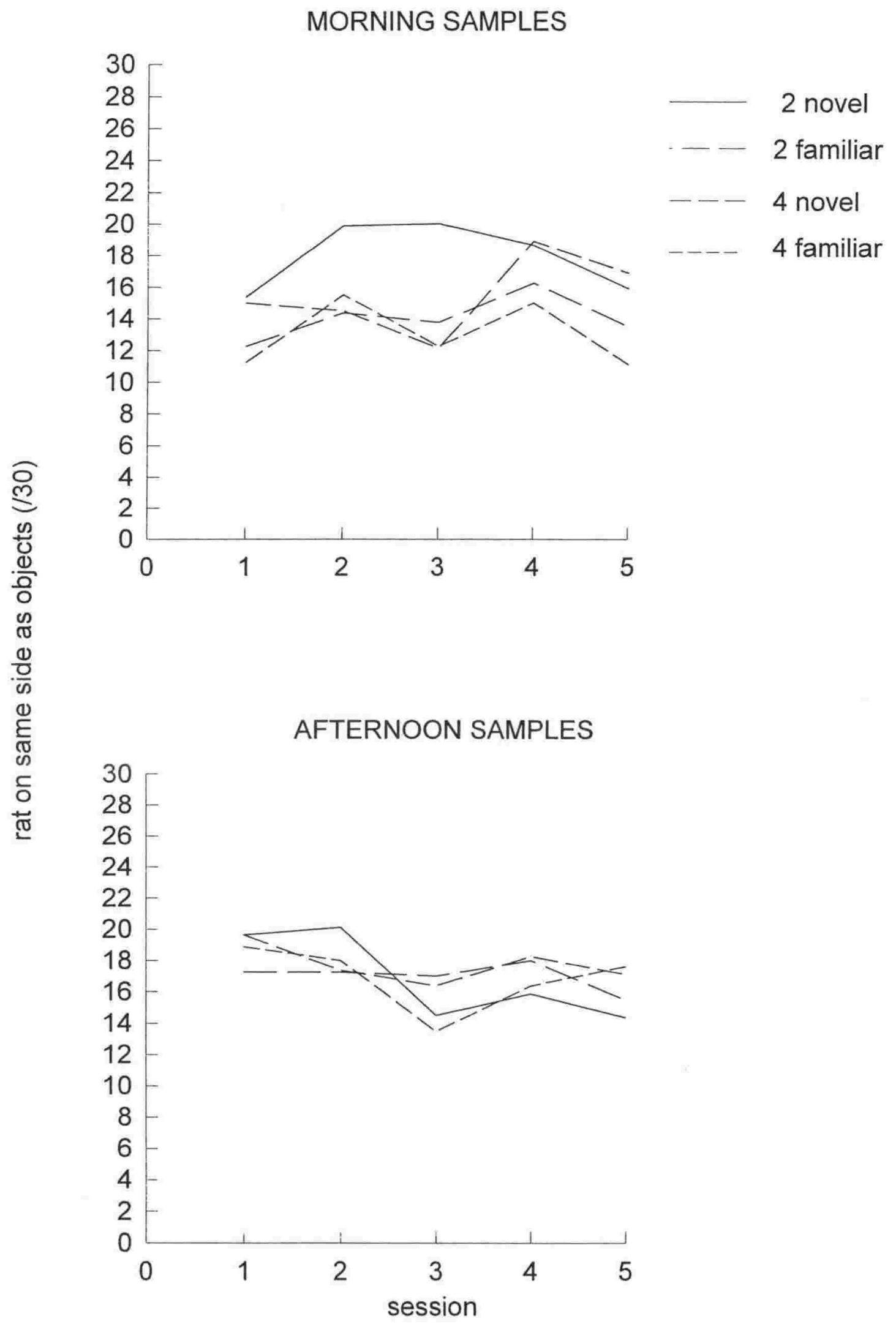

Figure 3.3 Mean time samples occurring on the objects side (/30) as a function of consecutive morning or afternoon point samples. 
would occur about $5 \%$ of the time (binomial p<0.059). Preferences of 28 or above were considered significantly different from chance.

Table 3.1 shows that individual scores out of 50 for six subjects. Wood shavings were significantly preferred $(>28)$ by all of the rats as measured by the access boxes and Tmaze. Preference for the wood-shaving option was also reflected in the group means. However, novel objects were generally not significantly preferred over an empty area, with an equal number of subjects scoring $<25$ and $>25$. Only a few individuals achieved a novel object preference score of 29 or higher (4/36), and all of the group means were less than 28 .

The T-maze estimates of preference for wood-shavings were significantly lower than those from the preference boxes (Friedmans ANOVA, $\mathrm{K}=0.58, \mathrm{df}=2$, Wilcoxon matched pairs, $\mathrm{T}=0 / 1, \mathrm{df}=5, \mathrm{p}<0.05)$. This was also reflected in that the rats mean preferences for wood-shavings which were above 28 only with data from the access boxes (and not from the T-maze). No clear preference was shown for novel objects. This data is not informative for further investigating the agreement of the three preference testing apparatus.

Table 3.1

Mean Choices for the Intervention out of a Total of 50. Choices $>28$ are Shown in Bold Type

OPTION

Wood Shaving

Large Access Box

Small Access Box

T-Maze
TIME

$\underline{9.30}$ 34

37

22
39

39

$\begin{array}{lllll} & & & & \text { Group Mean } \\ 43 & 43 & 42 & 44 & 40.83 \\ 40 & 43 & 46 & 30 & 39.17 \\ 31 & 28 & 32 & 33 & 29.33\end{array}$




$\begin{array}{llllllll}\text { Wood Shaving } & \underline{5.30} & & & & & & \text { Group Mean } \\ \text { Large Access Box } & \mathbf{4 7} & \mathbf{4 0} & \mathbf{4 6} & \mathbf{4 4} & \mathbf{4 2} & \mathbf{4 0} & \mathbf{4 3 . 1 7} \\ \text { Small Access Box } & \mathbf{5 0} & \mathbf{4 0} & \mathbf{3 2} & \mathbf{3 0} & \mathbf{3 4} & \mathbf{4 1} & \mathbf{3 7 . 8 3} \\ \text { T-Maze } & 24 & \mathbf{3 1} & \mathbf{3 0} & \mathbf{2 9} & \mathbf{3 0} & 26 & \mathbf{2 8 . 5} \\ & & & & & & & \\ \text { Novel Objects } & \underline{9.30} & & & & & & \text { Group Mean } \\ \text { Large Access Box } & 12 & 22 & \mathbf{3 6} & \mathbf{3 7} & 27 & 20 & 25.67 \\ \text { Small Access Box } & \mathbf{2 8} & 25 & 21 & 21 & 27 & 21 & 23.83 \\ \text { T-Maze } & 26 & \mathbf{3 0} & 19 & 23 & 26 & 23 & 24.5 \\ & & & & & & & \\ \text { Novel Objects } & \underline{5.30} & & & & & & \text { Group Mean } \\ \text { Large Access Box } & 17 & 17 & 22 & \mathbf{2 9} & \mathbf{2 9} & 24 & 21.4 \\ \text { Small Access Box } & 34 & 20 & 17 & 22 & 23 & 25 & 21.05 \\ \text { T-Maze } & 24 & \mathbf{3 1} & \mathbf{3 0} & \mathbf{2 9} & \mathbf{3 0} & 26 & \mathbf{2 8 . 5}\end{array}$

\section{Discussion}

The rats displayed investigatory behaviour in the presence of manipulatable objects, but it was frequent only when the objects were novel, and the preference data indicated that the rats did not prefer to be in cages containing such objects. T-maze estimates of preference were consistently lower than those from the continuous access boxes. As such, although the T-maze and continuous access seem to provide similar data, the T-maze may be slightly less sensitive. Given that previous studies have found the opposite trend, it may be that the relative effectiveness of the two preference procedures depends on variables such as the enrichment object being assessed, or the species. Rats tendency to alternate spatial response might be one reason for the Tmaze to be less sensitive than the continuous access box in this case.

It is possible that even very brief increases in investigatory behaviour are beneficial to rats, but based on the current evidence there is not sufficient evidence to justify 
providing laboratory rats with arbitrarily chosen objects as enrichment devices. From here, there were two directions open for investigating possible ways to enrich the rat cage environment. One option was to look at the other aspects of enrichment (space, other rats and fixtures) to see whether these produced significant levels of preference (Experiment 3.2). The other option was to see whether small enrichment objects could be found, that the rats did prefer (Experiment 3.3). Small objects as enrichment items have a number of advantages in terms of immediate application and cost. 


\section{EXPERIMENT 3.2:}

Preference for the Dimensions of Enrichment

Manipulible objects are only a small part of the traditional enrichment condition. The other qualities that an enrichment condition normally has are increased space, a larger social group, and fixed cage dividers.

The traditional enrichment literature provides some indirect evidence about what aspects of the enrichment contribute most to its effects. This comparison has normally been done by producing partial enrichment conditions which include only some of the normal dimensions, and comparing them to the effects of a full enrichment condition. Objects are normally found to contribute to some of the effect (Forgays \& Forgays, 1952), while increased group size makes little or no difference (Bennett et. al., 1964; Krech et. al., 1966).

The situation would be made clearer by examining the preferences of the rats for all four of the dimensions present in the standard enrichment condition. The following experiment measured the rats preference (over an empty standard cage) for novel objects, a double sized cage, three familiar rats and four fixed wooden pillars. A final option included all of these interventions and was equivalent to the semi-enriched condition from Experiment 1. 


\section{Method}

\section{$\underline{\text { Subjects }}$}

The subjects were twelve Hooded Norway rats, six male and six female. They were six months to two years old, with an average of around eighteen months. They had variable prior experience ranging from being breeding stock, or serving in a range of operant experiments. The subjects were kept under standard conditions as described in Experiment 1. Adjacent pairs of rats were kept interchangeably so that groups of four rats would be reasonably familiar with each other.

\section{Apparatus}

The apparatus were the T-maze and large continuous access box from Experiment 3.1. The environmental conditions provided were: one double sized cage, one cage containing three rats, one cage containing four fixed wooden pillars and one cage containing four novel objects. The double sized $\left(1800 \mathrm{~cm}^{2}\right)$ cage was made by joining two cages together and would meet Lawlor's (1989) standard dimensions for keeping adult pairs of either sex.

\section{$\underline{\text { Procedure }}$}

The procedure was the same as for Experiment 3.1 (Part 3.1.3) with the following exception. The number of sessions per condition was increased from five to six, thus a total of 60 observations were made. 


\section{Results}

If the rats' choices were random, then they would choose each option approximately 30/60 times. Based on a binomial probability distribution, choosing one option 36 or more times would occur less than five percent of the time. If the rats average score was 36 or more, the option indicated was consider to be preferred (binomial $\mathrm{p}<0.05$ ).

Figure 3.4 shows the arithmetic mean and standard errors for the 12 subjects. The means were representative of most of the subjects. This figure shows that most of the four separate enrichment options were not significantly preferred. This was with the exception of space as measured by the continuous access box (39.25), and for access to three rats in the T-maze procedure (36.4). However the four dimensions combined together (in the semi-enriched condition) were significantly preferred as measured by both procedures.

\section{Discussion}

The results show that few of the four 'single aspect' options were significantly preferred using any given testing method, and none were preferred as measured by both preference measures. However, the semi-enriched condition, which combined all of the other four options, was preferred as measured by both procedures. 

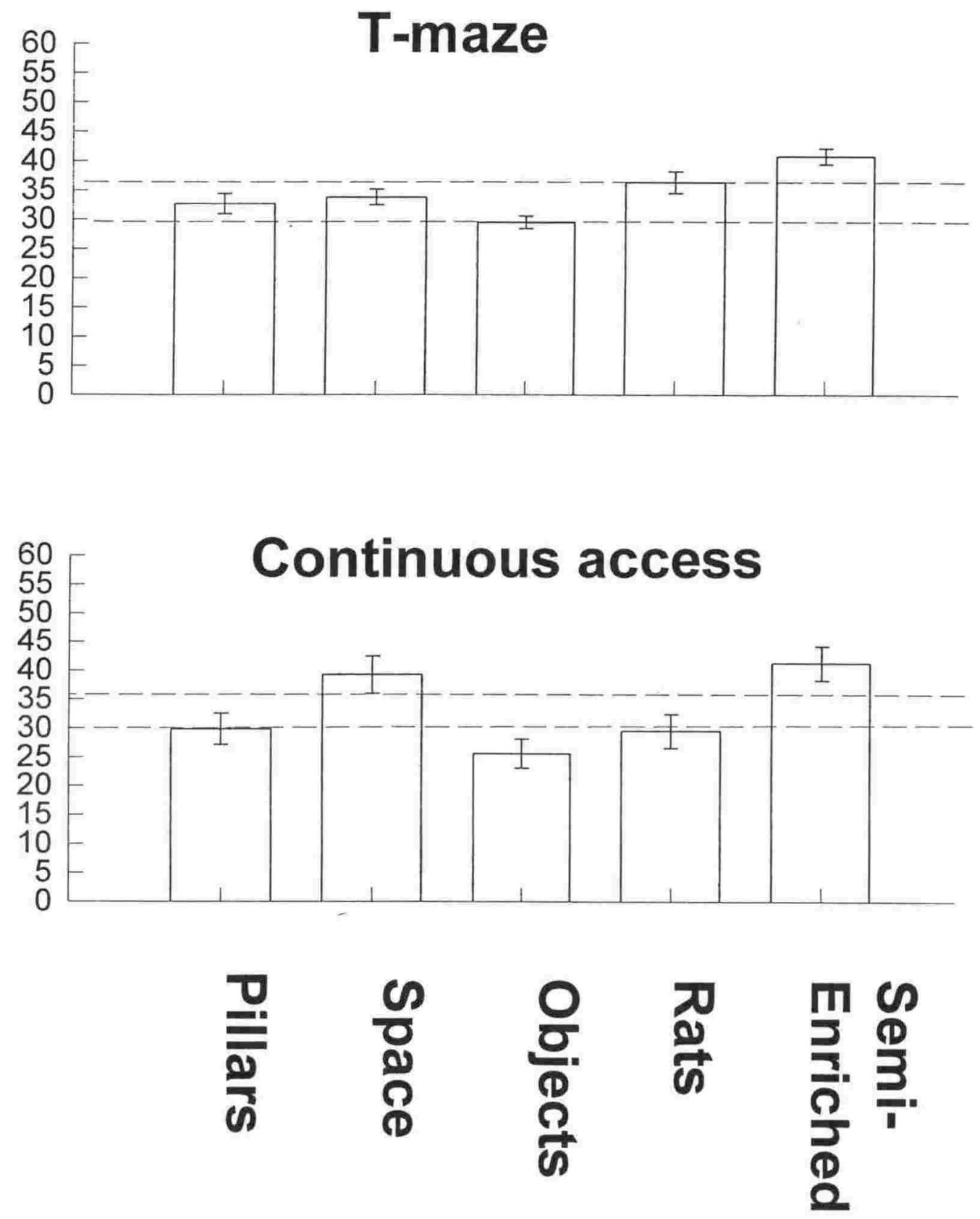

Figure 3.4 Mean contact preference for the four dimensions of semi-enrichment and the total semi-enriched condition. The lower dotted lines showed chance levels of performance (30/60) and the higher dotted line shows performance significantly above chance (36/60). 
There is one significant note of caution to be made when interpreting these results. With the continuous access box the 'space' and 'semi-enriched' option are twice the size of the empty cage. This means that a randomly wandering animal would spend two thirds of its time in the larger environment.

Unequal option spaces would have no effect on the T-maze results as the animals are not able to wander between environments, but it meant that there were two different ways to interpret the continuous access box results. First, the rat might have treated the empty cage and the alternate cage as distinct options, and chosen to be in one or the other. The distinct partitioning of the two areas might have lent itself to this treatment by the rats. This would mean that the continuous access box produced two significant results; for space and the semi-enriched cage. However, if the rats, as exploratory animals, made no real distinction between the two areas then the data relating to extra space and the semi-enriched environment are just on chance levels of $40 / 60$ (39.25 and 40.83 respectively). On balance the latter interpretation is probably more parsimonious.

This means that no significant results were produced using the continuous access box. This is consistent with Dawkin's (1976) and Hughes and Black's (1976) finding that the continuous access did not tend to detect any significant preferences even when the T-maze did. For the interventions that did not include double space (rats, objects and pillars) the T-maze gave much higher estimates of preference than the continuous access box. However, this difference might be due in part to the two procedures measuring slightly different behaviours rather than a difference in sensitivity per. se. 
So it would be prudent to continue using both procedures in tandem until there is more conclusive evidence.

Finally. the T-maze data suggested that rats do prefer access to a group of three rats, and the semi-enriched environment, over the empty cage. It was possible that the group of rats was preferred only in the T-maze because this method allowed the subjects to make direct contact with the companion rats. In the continuous access box this was not possible as the subject rats had to be free to move to the empty cage and back, while the other three rats had to stay on the 'enriched' side. This presumably reduced the enriching qualities of the social contact. When the T-maze was repeated with the three rats restrained behind a screen it returned a result of 35.25 which is below the level considered to reflect a significant level of preference (36). Thus, contact with rats might be more preferred than the continuous access box data indicates.

Experiment 3.1 showed the continuous access box gave a higher estimate of preference than the T-maze. This probably had to do with the use that the rats had for wood-shavings. Wood-shavings are likely to make a much more comfortable resting surface than bare plastic. The rats in the continuous access box might have chosen to come to rest on the side with wood-shavings, when left uninterrupted for the hour long session. The T-maze keeps the rats active by removing it from the environment every five minutes, and the two options are more equivalent to rats that are active. Based on this conclusion it might be that environments that facilitate rest would produce higher estimates of preference using the continuous access box, but options (such as pillars, 
objects and rats) that promote activity, would produce higher estimates on the T-maze. However, on balance it seems likely that the T-maze is the more sensitive method for estimating environmental preferences.

In the T-maze a higher preference was shown for the semi-enriched environment. This suggested that there are some conditions the rats will show a preference for. It also suggested that the effect of enrichment might not depend on any one of its ingredients, but on the interaction of the ingredients with each other (enrichment may be more than the sum of its parts). Aside from this the results suggest that the presence of a larger group of other rats might be an effective way of improving the environment. 


\section{EXPERIMENT 3.3:}

\section{Preferences for Cage Furniture}

Of the four separate dimensions of environmental enrichment, a larger group size was the only option that the rats from the previous experiment showed a statistically significant preference for. However, enlarging the group size necessarily involves increasing the size of the cage, and thus such an enrichment may be difficult to provide in laboratories designed to accommodate smaller cage sizes.

The current experiment enlarged the search for an effective enrichment device that could be incorporated into standard housing. This was done in order to discover whether there are any short term changes that can be made whilst investigating the possibility of using a larger group cage at some time in the future.

Experiment 3.1 showed that arbitrarily chosen novel objects were not preferred by the rats. However, objects chosen in a less arbitrary way to fulfil specific behavioural functions, might be more successful. Some behaviours that rats show even in laboratory cages are resting, chewing, perching and tunnelling. Objects were chosen to provide some kind of substrate for the performance of these behaviours. 


\section{Method}

\section{$\underline{\text { Subjects }}$}

The subjects from Experiment 3.2 were used when that experiment came to an end.

\section{$\underline{\text { Apparatus }}$}

The cage furniture used included; 4 sheets of tissue paper (each being $20 \mathrm{~cm}^{2}$ )one handful of shredded paper, a nest box made from a large plastic pottle or a coffee tin with an $8 \mathrm{~cm}$ entrance hole, two sticks, four walnuts, a wooden platform, a PVC tunnel ( $8 \mathrm{~cm}$ diameter and $15-20 \mathrm{~cm}$ long) and three tunnels glued together in a pyramid. The T-maze and large continuous access box used were the same as shown in Experiment 3.2 .

\section{Procedure}

Each of the twelve rats had their preference for the environments assessed using the Tmaze and large continuous access box according to the procedure described in Experiment 3.2. Order of testing was counter-balanced for apparatus and the environment presentation was partially counter-balanced. Also, when T-maze choices and access box observations for tin nest boxes and shredded paper were made, the behaviour shown by the rats was categorised. In the final stage of the experiment, preference was assessed for the tin nest box versus the shredded paper. 


\section{Results}

Figure 3.5 shows arithmetic means and standard errors for the twelve subjects scores (out of 60). A score of 36 or higher was considered significantly different from chance, based on a binomial distribution $(\mathrm{p}<0.05)$. The first cage additions assessed were tissue paper, plastic containers, wooden sticks, platforms and plastic pipes. Mean data indicated that the tissue paper was significantly preferred as measured by the continuous access box, while the platform was significantly preferred as measured by the T-maze. Further testing was done to assess shredded paper, tin nesting boxes and walnuts. The paper and nest boxes returned mean preferences on both apparatus which were significantly higher than chance.

The two most preferred options (shredded paper and tin nest boxes) were further investigated. Observations of shredded paper and nesting box use by the rats are shown in Figure 3.6. The rats spent $25 \%$ of the time at rest on the paper, and $12.5 \%$ of the time manipulating the paper. With the nest boxes; they were inside the box $35 \%$ of the time, and leaning or standing on it $10 \%$ of the time. When preference was tested for paper versus nest boxes the nest boxes were significantly preferred (continuous access: 37.2 sd 3.2, T-maze: 37.6 sd 2.2). 


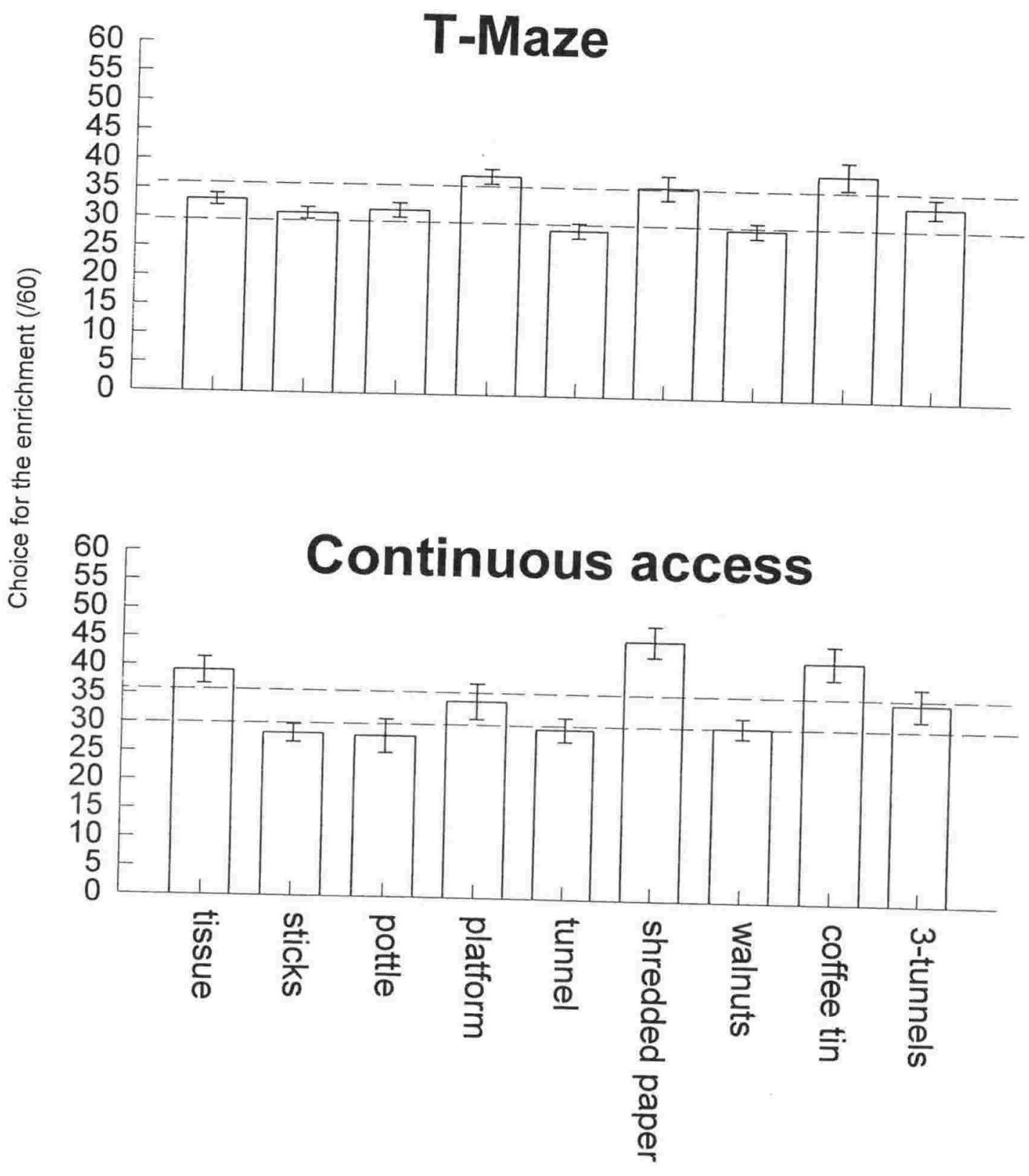

Figure 3.5 Preference for nine cage options as measured by the continuous access box and T-maze (showing standard errors of the mean). 


\section{Shredded \\ Paper}

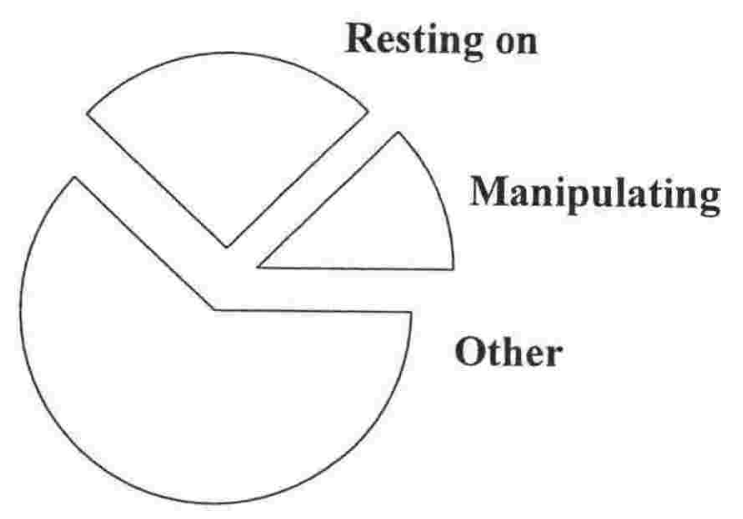

\section{Coffee}

Tin

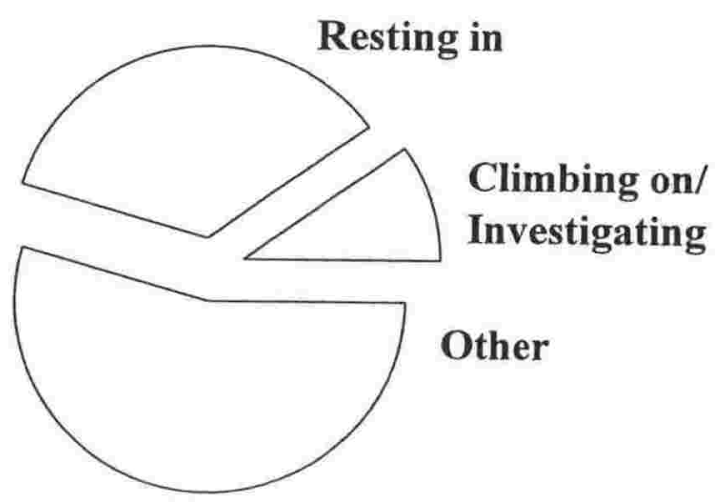

Figure 3.6 Behaviours shown by rats in the presence of nest boxes and shredded paper, mean percentage time spent in each activity as observed in five minute time samples. 


\section{Discussion}

Most of the objects provided did not result in the rats preferring the cages that contained them. The four exceptions were; tissue paper, shredded paper, nesting tins and platforms, with only the nesting box and shredded paper being significantly preferred in both appartus.

Substrates for resting returned higher preference estimates in the continuous access box than in the T-maze. For example, the tissue paper was significantly preferred as measured by the continuous access box but not the T-maze, and the platform for climbing and perching was significantly preferred as measured by the T-maze only.

The uses that the rats make of the furniture were only putative in most cases.

However in terms of the shredded paper and nest boxes some observations were made that suggested the rats did construct nests with the paper, and sit inside the nest boxes. Rats did not consistently prefer environments that provided opportunities for chewing and tunnelling. This may be because these behaviours are not highly valued, or because the objects provided did not allow the rat to properly perform these behaviours. For example, the rats lack of preference for the plastic nest box did not reflect a lack of preference for nest-boxes per. se., as the tin nest box was preferred. Likewise tissue paper was not as strongly preferred as shredded paper. Other forms of chewing or tunnelling substrate might be preferred in later experiments. Likewise, objects that allow climbing, swinging or some other behaviour that was not investigated might be preferred as highly as the nesting materials. However, this does 
not detract from the clear preference for the nesting material, or the recommendation that these should be provided to rats kept in standard cages. When the rats had to choose between the shredded paper and the nest boxes they showed a significant tendency to opt for the nest boxes. The preference for platforms as measured by the T-maze suggests that this should be investigated as the second next promising option, ahead of the plastic pipe tunnels that are normally recommended by welfare guidelines.

The experiments that follow assess the rats preferences for larger environments that can contain larger group sizes and higher degrees of environmental complexity. There was also the lasting concern that there is more to the rats preferences than those behaviours which occur frequently enough to bias choice and show up in a high proportion of the samples. There is the potential (as outlined by Duncan, 1978 and Matthews, 1994) for behaviours that are shown only occasionally (thus not producing high preference scores) to also be important. This led to a move to using a more sophisticated method of preference assessment in the remainder of the experiments. Chapter 4 explores the use of economic demand theory to assess the rats reactions to a range of enriched cages. 


\section{Chapter 4}

\section{Economic Demand Theory}

\section{Replacing Preference with Demand}

The preference data from the experiments in Chapter 3 led to some suggestions about how to house laboratory rats. However these experiments also raised questions that might be better answered by making use of economic demand theory (Hursh, 1980; 1984). There are two main reasons for using demand procedures. One is that demand procedures might reduce the difficulties that result from assessing a large number of possible environments (Section 4.1). The other is that there are limitations with the Premackian theory of reinforcement that underlie preference tests, that do not apply to demand procedures (see Section 4.2). The key aspects of behavioural economics are outlined in Section 4.3, and Section 4.4 addresses some major issues and concerns that arise from using demand procedures.

\section{1) Comparing Multiple Environments}

When two environments are significantly preferred over an empty cage, one may still be preferred significantly over the other when they are compared directly. Also, the result of a direct comparison cannot be easily predicted by looking at the relative degree of preference versus the empty cage (Bradshaw \& Poling, 1991). This would suggest that every preferred environment would have to be directly compared with every other preferred environment in order to appreciate which would be of the greatest benefit. To perform such comparisons would be very cumbersome and prohibitively time consuming. It would be better to be able to form a preference hierarchy in the first assessment, without the need for direct comparisons. 
Demand procedures might provide a hierarchy of preference that does not require extensive cross-comparisons.

\section{2) Limits of Premackian Theories of Reinforcement.}

The behavioural economic approach can also reveal a greater depth of information than simple preference procedures. Demand procedures are based on making the animal perform a response in order to gain access to an alternative environment. The number of responses required is varied, and the effect on the animals response rate is taken to reflect the subjective value of the alternative environment. It is often found that two environments that are responded to equally at a low response requirement, will produce very different responses when the price is increased (for example, Hogan et. al., 1970; Roper, 1973). The responding at low requirements is similar to preference, as determined by the preference procedures used in Chapter 3. However, the extent of responding at higher response requirements provides data that might distinguish between environments that are equally preferred (at low response requirements). In essence, demand procedures provide information about both an animal's preference for an environment, and how much it demands this option when it comes at a price.

Another situation where demand would help is with an environment that the rat does not choose to be in often (does not prefer) but will work very hard to maintain this occasional presence (demand). Simple preference studies have the potential to be misleading because they only take into account the time that the animal spends in different environments, when small amounts of time in some environments might still be highly valued (and vice versa). 
The following sections briefly outline the development of the economic analogue, and the problems with its use, before describing how it was used in the following experiments.

\section{3) Early Economic Demand Theory}

Over twenty years ago researchers began to study how hard animals would work, to obtain non-food rewards. They found that when food was provided according to a fixed ratio schedule (FR), animals increased their responding as the response requirement increased. When other commodities were provided the animals generally maintained a steady level of responding as the response requirement increased. Thus the animals essentially obtained fewer reinforcers for the same amount of responding (for example, fighting fish and mirrors for aggressive display, Hogan et. al, 1970; mice and nesting material, Roper, 1973).

Subsequently, demand theory from economics was used as an analogy for describing data produced using this kind of procedure (e.g. Hursh, 1984). The animals' responses were equated with work, the FR with price and the environment worked for, the commodity. The number of times the animal earns access to the commodity, is graphed against the price of the commodity during that session, using a log scale. The animals demand for a reinforcer is measured as the slope of a line fitted to these data. Demand is described as elastic if the slope is steeper than -1 or inelastic if the slope is shallower than -1 . Inelastic demand is thought to indicate that the commodity is a need and elastic demand that it is more likely to be a luxury. The provision of environmental needs would be more likely to improve an animal's welfare than the provision of luxuries. 
The study of economic demand has, so far, produced only a moderate number of applied experiments. This may be partly because the demand procedure is difficult to automate and often time consuming when used with commodities other than food. There is also a certain amount of disagreement on points of theory. This makes the use of demand more contentious than the use of simpler procedures like preference.

\section{4) Problems and Procedures}

The wholesale application of economic demand theory and its methods and analysis have produced a number of difficulties for those interested in its application to animal welfare. The most problematic issues for animal welfare researchers relate to quantifying the parameters of demand, the use of non-food commodities, session length, and the distinction between open and closed economies. These problems have become more pressing since the advent of a faster procedure (involving daily price increments) has enabled researchers to use a larger number of commodities within a reasonable time frame (Hursh et. al., 1988).

\section{Non-food Commodities}

There are practical problems when demand procedures are used to assess commodities that cannot be quickly consumed. Most demand experiments have used food reinforcement, as this can be delivered immediately into the operant chamber and is quickly consumed. It is interesting that many of the experiments that apply demand theory to a non-food commodity, used drugs as a commodity, as drugs are one of the few options other than food that can be provided as instantaneously as food (Hursh, 1991; English et. al., 1995 for example). 
In the field of animal welfare researchers often want to assess demand for commodities that are unwieldy, both in the space they occupy and the time the animal needs with them in order to perform the behaviour that is reinforcing (nesting material, climbing frames etc.). For most environmental variables it is necessary to allow fairly long periods of consumption time, even though this introduces a tricky confounding variable that has yet to see much investigation. One study settled on five minutes (Nicol \& Sherwin, 1998), and this period is commonly used in preference studies (Dawkins, 1977, 1978, 1982; Woodgush \& Vestergaard, 1991).

\section{Quantifying Demand}

Generally, demand has been measured by choosing a set of FR values (prices), gathering the consumption data, and fitting a straight line to the resulting data (for example Sherwin \& Nicol, 1997). If the slope of the line was steeper than -1.0 then it was called elastic (indicating the commodity is a luxury), otherwise demand was deemed inelastic. However, an animal's demand for a commodity can move from inelastic to elastic as the price increases; producing a downward sloping demand curve (Hursh, 1980). Unless the price range is restricted it seems that this will always be the case, making it impossible to use a straight line fit. Leaving out the high price range or elastic section of the data, and using a straight line fit to the remainder, might over emphasise the inelasticity of the data in many cases.

When commodities that are in moderate demand at low prices are tested until extinction, a curved pattern of data will result. This data can be described by a negatively accelerating fitted line (Equation 4.0, below). The point on the x-axis where this fitted line has a slope of 1 , can be used to indicate demand elasticity in much the same way as the linear slope of a straight line fit (Hursh et. al, 1988). 
Equation 4.0

$\log Q=\log L+b(\log P)-a P$

Hursh et. al., 1988

$Q$ is the number of reinforcers consumed and $P$ is the cost of each unit of reinforcement (FR). The free parameters of the curved line are $L, a$ and $b . L$, is the height of the line at the price 1.0. $L$ quantifies the overall height of the line (Hursh et. al., 1988). $L$ reflects something like the unconstrained preference as used in the experiments in Chapter 3, where no cost had to be paid to enter the alternative environments. $b$ is the initial slope of the line, and $a$ is the rate of its change. $a$ and $b$ together can be used to calculate pmax, which is the FR at which the slope is equal to -1. pmax is calculated using the following equation.

Equation 4.1

$$
\operatorname{pmax}=(1+b) / a
$$

Foster et. al., 1997

pmax directly reflects the animal demand for an environment by indicating the FR at which it stops increasing its responding with the increasing price, and therefore starts getting less of the commodity than it would prefer. Other values, like the highest FR at which a commodity is obtained, can be used to encode demand if pmax is not able to be calculated.

Some recent studies have continued to use a restricted $\mathrm{x}$ range and straight line fits (for example Sherwin \& Nicol, 1998). However, testing to extinction and using a curved fit is likely to be the better option for the current study (see Figure 4.1 which shows demand for chocolate by Subject 1 in Experiment 4.2). 


\section{Open and Closed Economies}

In the field of animal welfare science, the main impediment to using demand procedures is the widely accepted belief that a laboratory must maintain a fully closed economy in order to produce valid demand curves (for example Hursh, 1980). This requires that the commodity that the animal earns in the chamber is not available anywhere else. If this assumption is correct it would indicate that demand experiments cannot normally be used to determine the relative value of cage enrichment options.

The reason for this assumption is that every demand experiment uses two linked environments. In 24 hour experiments they are the work area where the animal performs the operant, and the area where the commodity is consumed. In the case of food these two areas are only separated by time, because food is delivered into the experimental chamber. In most other cases the work and consumption areas are also separated by space. The commodity is the environment the animal works to gain access to, but equally it is working to escape from the working area. Thus both environments contribute equally to the degree of demand shown. When short sessions are used, the working cage and the home cage are separate areas. It is not clear which of these would have a greater effect on demand. The working cage might represent an aversive environment the rat wanted to escape in the short term, and the home cage could contribute to longer term deprivation that also impacts on demand. 


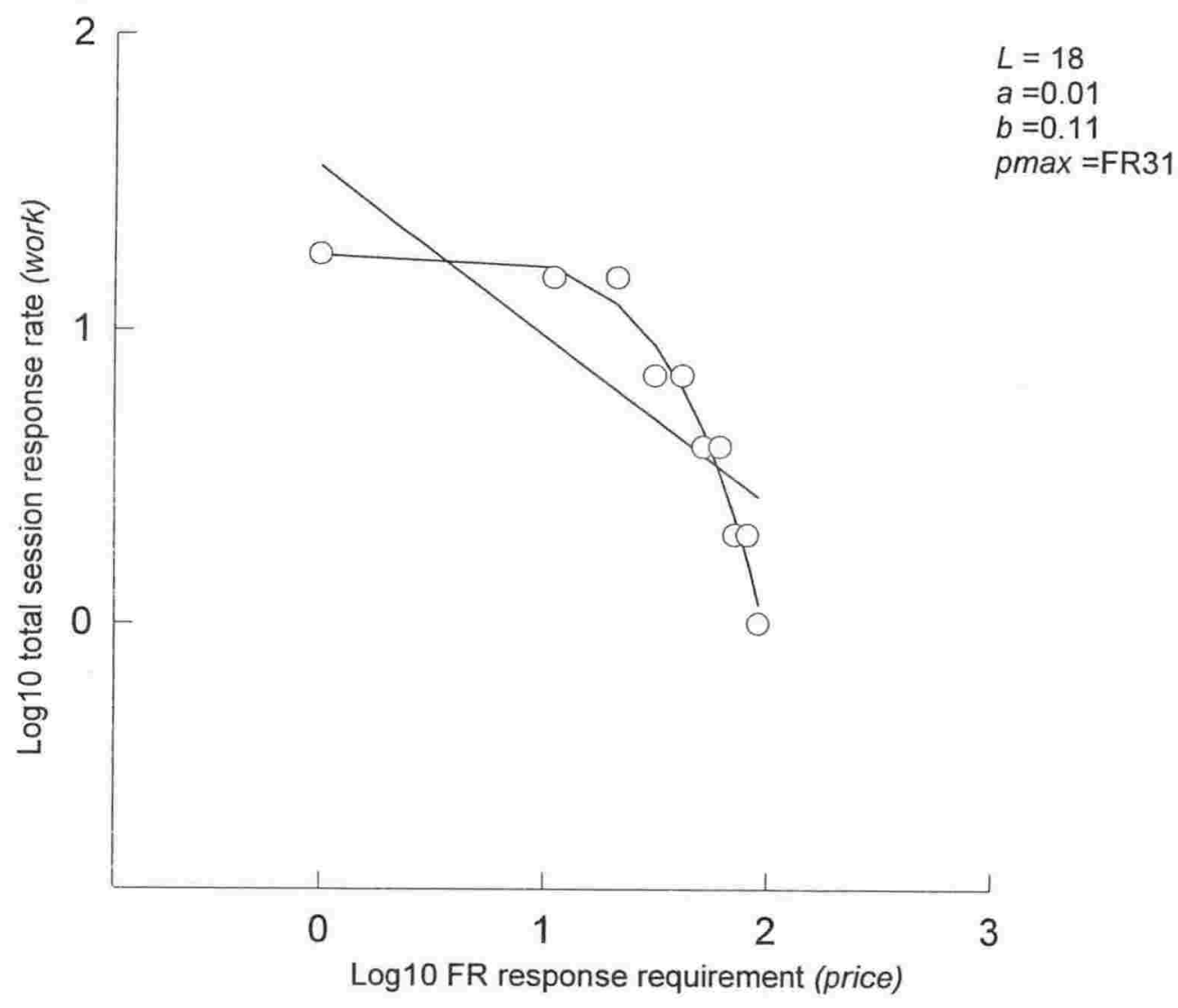

Figure 4.1 Demand data demonstrating straight and curved line fits (least squares regression and Equation 4.0). The data are from Subject One in Experiment 4.1.3, the first data collected from FR2 to extinction (one line) with chocolate as the commodity being responded to on an FR schedule. 
The main difficulty with maintaining a closed economy is that if the commodity worked for was open space, the home cage could not contain any space. This is obviously impossible, and it is difficult for most other interventions that might improve an animals welfare. In the standard home-cage there are manipulatable objects (sawdust, the rats tail etc.), fixed objects (bars, water dispenser) and other rats. Besides, in an applied setting we want to know what would improve the animals environment, which is by definition the things it would work for over and above what is already available to it. Even if it were possible to determine an animal's absolute demand for different commodities this would not tell us which of these it already had in sufficient amount under standard conditions and which was in most need of improvement. So, a closed economy is neither possible, nor desirable.

The main reason that closed economies are thought to be necessary is that demand curves generated by animals in open economies are extremely elastic (as stated in Lea \& Roper, 1977; Hursh, 1980; Hursh, 1984). This is thought to be because the commodities provided outside of the session are fully substitutable for commodities obtained within the session (Lea \& Roper, 1977; Lea, 1978; Hursh, 1980). However, this assumption is based almost entirely on a single three subject study by Lea and Roper (1977), and the conclusion is not beyond doubt. The acceptance of the distinction between economies might owe more to the place it has in the original economic theory, rather that its value in application to animal behaviour.

A series of experiments by Timberlake (Timberlake, 1984; Timberlake \& Peden, 1987 etc.) deal very thoroughly with the expected effects of open and closed economies on the behaviour of rats. Their findings show that rats do not substitute food during the session with 
food available at other times. Thus, the nature of the economy had no effect on behaviour within the session. Open economies reliably produced the same mixed curves that the closed economies did. This finding directly contradicts theories that distinguish between open and closed economies.

It is possible that the economy does have some effect on demand in some circumstances, but it probably does not normally produce the rampantly elastic curves that pure economic theory would predict. One would hope that the rats have some ability to substitute between the experimental session and the home cage. If they could then their demand data could be taken to reflect how their cages should be improved. If they could not substitute commodities across time (i.e. between the home cage and the experiment) then demand data would only reflect general preferences, and it would remain unclear how to best improve the existing home cage which would already satisfy some of these preferences to some extent.

\section{The Advantage of an Open Economy}

Using an open economy allows the whole range of possible prices to be used without risk of extreme deprivation (i.e. starvation) occurring at the higher prices. Timberlake (1984) extends this point by suggesting that in a closed economy for food the body-weight of the animal reduces at the higher prices, and that this introduces a strong confounding variable. It is logical to assume that the rats demand for food would increase as its body weight decreased, thus the demand line would not describe a single state of motivation, but one that increases as the FR does, thus exaggerating the inelasticity of the resulting demand curve. 


\section{Session Length}

Finally there is the issue of session length. In the following experiments the session was short (40-55 minutes). Long sessions have the advantage of making an open economy more closed because a greater proportion of the food would be obtained within the session. However, a different arrangement was used here to increase the independence of food within and outside of the experimental session so that a short session could be used. The food provided in the session was a preferred food, in this case sweetened condensed milk, while the supplementary feed, was the less preferred, diet pellets. These two commodities are not identical and will therefore be only partially substitutable (Lea \& Roper, 1977). So although the economy is unarguably open for food, it is closed for condensed milk. Elastic demand should only occur when the within session commodity is of equal or lesser value to that available in the home cage. Thus simply using a preferred food should safeguard against excessive elasticity in the data.

Short sessions are also not generally favoured for demand work because they limit the subjects 'income' (total presses that can be accomplished during the session). It is arguable that this is not important, as it is recognised that animals preferences are relative. That is, they indicate only which of the available options is 'better', rather than whether any single environment is 'good' (for example, Appleby, 1997). Demand studies also produce relative data, although the comparison being made is less obvious. Judicious tampering with the experimental parameters would allow an experimenter to produce almost any demand slope even for food. What is crucial is the relative demand found for different commodities, tested using the same procedure. An income limit would at the most produce a slight ceiling effect in some cases. 
The Current Economy

Therefore, the economy operating in these current studies is a 'preferred food' (quasi-closed) economy. It is likely (on several grounds) to produce data that approximates that of a closed economy. Similar economies have already produced valid mixed demand curves. When hens work in long or short sessions for barley, and are post fed to $85 \%$ of their free-feeding weight with pellets, they were found to produce data that was similar to that from an equivalent closed economy (Foster et. al., 1997).

The present experiments used the demand procedure to assess the effectiveness of altered cage environments, much as the preference tests were used in Chapter 3. However the first experiment was designed to address any lingering concerns about the use of a short session, quasi-closed economy procedure for this purpose. 


\section{EXPERIMENT 4.1:}

The Effect of Economy on Demand Intensity and Elasticity in Short Sessions

The present study was conducted to assess whether a short session demand procedure, performed under an open economy, would produce interpretable data. Experiment 4.1 also assessed whether demand during the session is affected by the presence of the commodity (or its substitutes) in the home cage. The optimal finding would be that demand elasticity is affected, but not to the extent that the data becomes fully elastic over the whole price range. If this were the case, the rats would show the highest demand for those interventions that they value and that are currently missing from their home cage. This would allow demand data to be used directly as an indicator of which cage improvements would be best introduced.

Subjects worked within the session for sweetened condensed milk but feeding in the home cage was varied to produce three different conditions. Firstly the rats were given $a d$. lib. access to rat pellets (Condition A), then restricted to $85 \%$ of their free-feeding weights and post fed with a limited amount of pellets (Condition B), and finally provided with ad. lib. pellets and $a d$. lib. sweetened condensed milk (Condition $\mathrm{C}$ ); in an ABAC design. It was expected that Conditions A and B would produce mixed demand curves typical of closed economies but Condition B would have greater inelasticity. This would be because pellets are probably partially substitutable for milk, as a less preferred form of food. Only Condition C might show less inelastic demand, or the completely elastic demand that reportedly results from an open economy (Lea \& Roper, 1977). This would be because condensed milk is available both in and outside of the session. 
This experiment deals incidentally with another issue. If the results do demonstrate mixed demand curves when the rats are free fed on pellets, then the usual practise of keeping these animals at $85 \%$ of their free-feeding body weight might be questioned. If food deprivation is not necessary to motivate the animals responding for food during the session, then researchers would need to look at whether it is justified on any other basis.

\section{Method}

\section{Subjects}

The subjects were six female Hooded Norway rats aged forty days at the beginning of autoshaping.

\section{$\underline{\text { Apparatus }}$}

The subjects were housed under standard conditions as described in Experiment 1. The response chamber was the same as that described in Experiment 2 except that it contained only one lever on the right hand side.

\section{$\underline{\text { Procedure }}$}

The rats were auto-shaped to press the lever for sweetened condensed milk and were then exposed to a VI30 s schedule for two days. Rats then commenced the experimental conditions. Experimental sessions lasted 45 minutes, and the sessions operated on every second day. The first session with FR1 re-established lever pressing and is not presented in the data, the second session used FR2 and the FR requirement increased by two for each 
session until the rat received less than eleven reinforcers per session for two consecutive sessions. This process was repeated three times for each rat (Condition A). During autoshaping and Condition A rat pellets were available $a d$. lib. in the rats' home cages. Then the rats weights were gradually reduced to $85 \%$ of their free-feeding weights and three more lines were generated in the same manner (Condition B). Then pellets were provided freely again and three more lines generated (to replicate the baseline Condition A). Finally, free access was given to sweetened condensed milk in the home cage and a final three lines were generated for each rat (Condition C).

\section{Results}

Equation 4.0 provided a good description of the data, and met the requirement that it be a good visual fit to the data. Also this equation produced parameter values within an acceptable tolerance range (5\%), within 100 iterations, (with starting values of 1.0$).{ }^{1}$ The tables in Appendix 4.1 show the parameters of the lines fitted to the individual data $(L, b$ and $a$, and pmax).

Figure 4.2 shows all of the data from Rat 1 . This subject was chosen arbitrarily as an example. The graphs on the left show all of Rat 1's data points, grouped according to condition. The large figure on the right shows lines fitted to the data from the three conditions.

1 This criterion was used in all of the experiments in Chapter 4. 
All Data

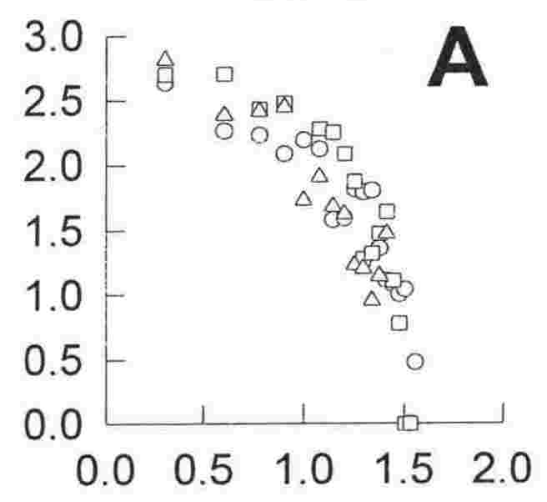

Fitted Lines
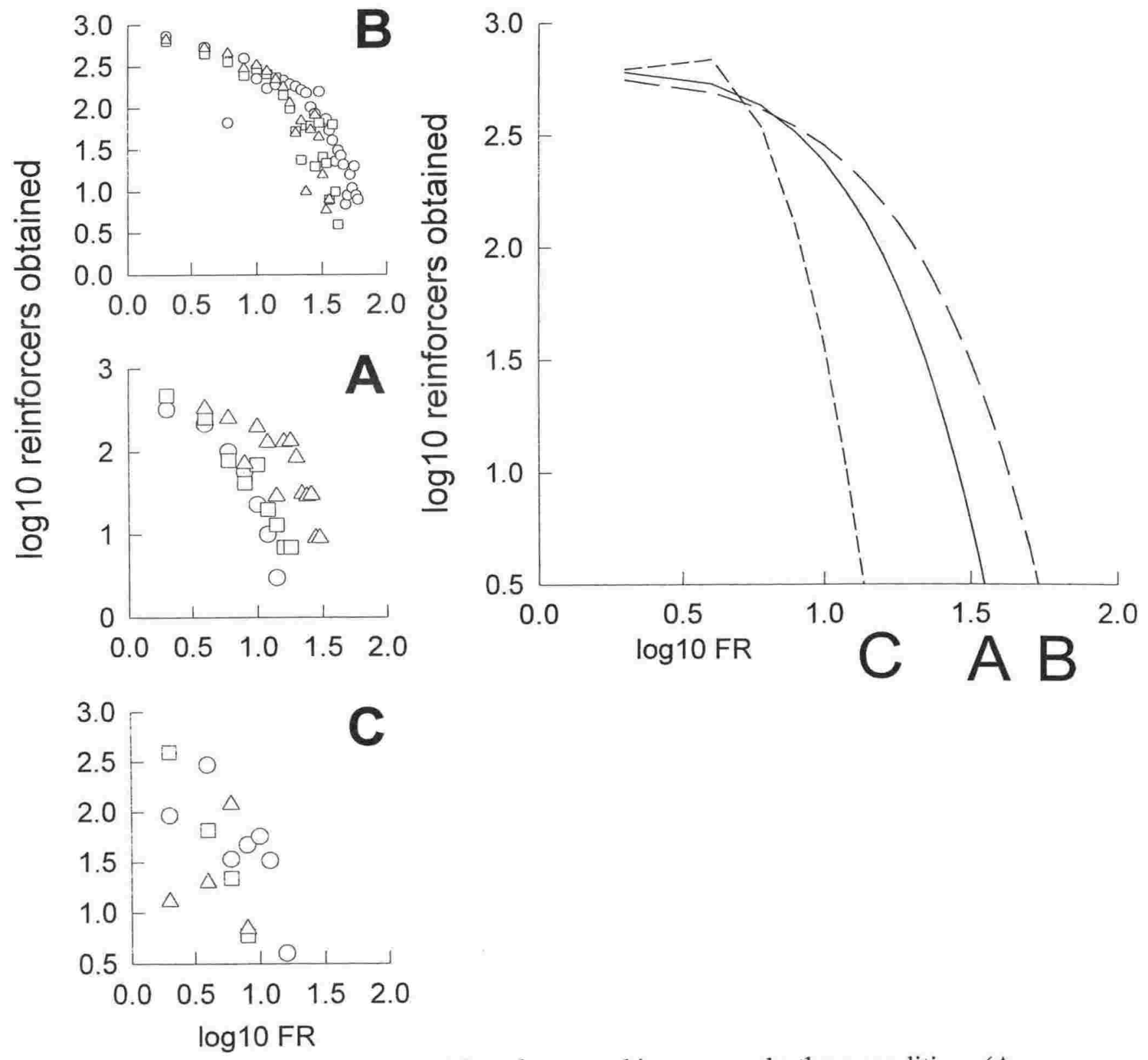

Figure 4.2 The demand curves and fitted lines for one subject across the three conditions (A, B and C). Different symbols are used for each of the three lines generated under the condition that the figure relates to. The fitted lines (shown in the right hand graph) are based mean parameters of lines (Equation 4.0) fitted to the individual 
Line B represents the laboratory practice that is used at this laboratory, where the animal works during the session for a preferred food and is post-fed with complete diet pellets. The line fitted to this data is a mixed curve with demand moving from inelastic to elastic as the FR ratio increases. It could not be said that the data is always inelastic (with a slope more negative than -1 at all ratios) during this condition, (as shown in Figure 4.2 or in the individual data for any of the rats shown in the Appendix 4.1).

Line A was generated when the rats were fed $a d$. lib. with pellets rather than restricted to $85 \%$ of their free feeding weights. Demand dropped off slightly sooner under these conditions but the basic shape of the curve is still mixed. This is reflected in the downward sloping curve with a pmax value smaller than for line B but still greater than zero. Of the 18 separate demand curves generated under Condition A, only two lines were fully elastic (see Appendix 4.1). One of these is the third line for Rat 1 (shown as the triangles in the top left graph) which just fails to meet the criterion for a mixed curve. Condition $\mathrm{C}$ is when the preferred food was also freely available in the subjects' home cages. The data under Condition C condition were less consistent than under Conditions A and B. In general demand was much more elastic in that the demand lines reaches a slope of -1.0 at a lower FR price. This should be reflected in lower pmax values for Condition C.

Figure 4.3 shows the mean intensity and elasticity of demand across the four rats that completed all three conditions. (Two rats died of a respiratory illness immediately prior to the commencement of Condition C; their data are present in the Appendices but does not contribute to Figure 4.3). The intensity of demand is reflected in the overall elevation of the demand curve and measured by the parameter $L . L$ (amount consumed at the cheapest price) 

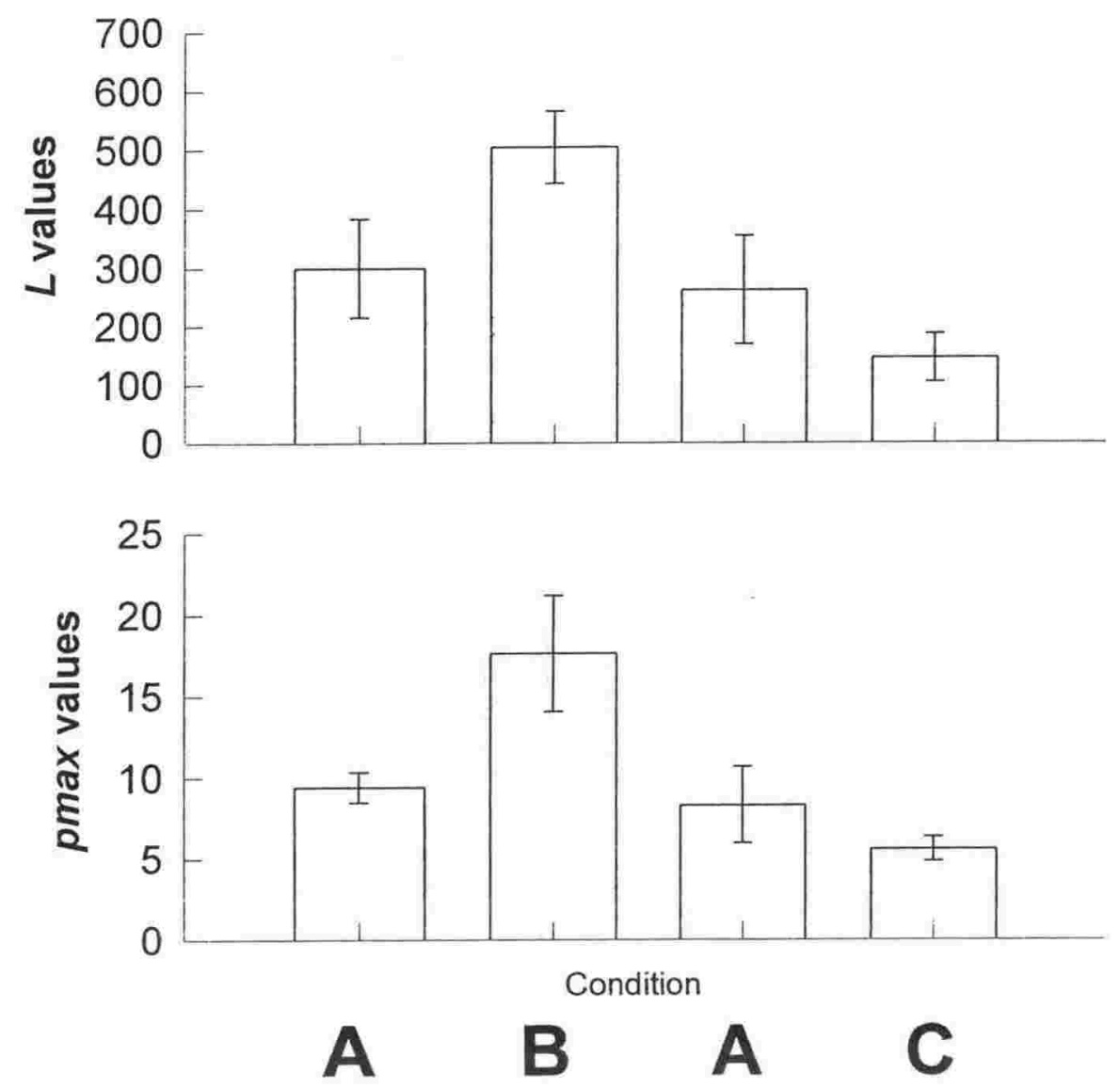

Figure 4.3 The mean values of $L$ and pmax for the fitted line of 12 demand curves of four subjects, for all conditions. Condition $\mathrm{A}=$ free feed pellets; closed economy condensed milk, Condition B $=85 \%$ body weight on pellets; closed economy condensed milk and Condition $\mathrm{C}$ = free feed pellets; free fed condensed milk. Standard error bars are shown. 
should be roughly equivalent to preference (amount consumed under conditions of free access). Figure 4.3 was based on an average of a representative data line from each subject (the median of the three lines collected for each subjects under each condition, each line being the response rate from FR2 to extinction).

$L$ values were highest in Condition B when access to pellets and milk was most restricted and thus most similar to a closed economy. In other words these subjects displayed relatively high preference or intensity of demand in this condition. $L$ was somewhat lower under Condition A when pellets were freely available in the home cage, and lowest under Condition $\mathrm{C}$ when both pellets and milk were freely available in the home cage. There were significant differences in $L$ across the three conditions (Friedman's ANOVA, Chi Sq=6.90, df=2, $\mathrm{p}<0.05)$

Elasticity is the degree to which the obtained amount of commodity per session reduces with increases in FR Ratio. It is shown here by the pmax and reflects the persistence of an animal's preference when a cost is incurred. pmax is the FR ratio when the demand moves from inelastic to elastic. It is the point beyond which the rat no longer increases its response rate sufficiently to maintain its consumption rate.

Under partial access to pellets (to $85 \%$ of body weight - Condition B) pmax occurred at approximately FR18. When the economy for pellets was open pmax occurs earlier (FR9 for the first replication and FR8 for the second). When the economy for condensed milk was open the mean pmax falls to FR6. The pmax data follows the same pattern as for the $L$ parameters. The conditions where the rats responded most at low prices (high intensity of 
demand), were also the conditions where the demand was maintained as FR increased (inelastic). These differences in pmax across condtion were statistically significant, (Friedman's ANOVA, Chi $\mathrm{Sq}=6.50$, df $=2, \mathrm{p}<0.05$ ).

It is clear that opening the economy for the two types of food results in a drop in overall elasticity. However the pmax remains measurable and positive, indicating that the demand curves are mixed rather than entirely elastic.

\section{Discussion}

This experiment demonstrated that the short session, open economy, procedures did generate mixed demand curves. Intensity and elasticity of demand varied according to the availability of pellets or condensed milk in the home cage. The great majority of the demand curves under all conditions were mixed rather than inelastic at all points, but the degree of elasticity depended partly on the availability of the commodity in the home cage. This is an ideal situation for the measurement of demand as an indication of how to best improve the rats' welfare. It means that the data showed the mixed form so that a uniform form of analysis can be used based on the negatively accelerating curve of Hursh et. al. (1988).

In this case only one commodity (condensed milk) was assessed. When this commodity was plentiful in the home cage, demand became less intense and more elastic. When a partially substitutable commodity (pellets) was freely available then demand was slightly less intense and slightly more elastic than when its availability was restricted. Thus a more intense and 
inelastic demand can be taken to indicate that a desirable commodity is available only in the experimental session. Less intense and more elastic demand would indicate that the commodity is less reinforcing, or that a substitute is available in the home cage. In such a case another commodity would perhaps make a more effective improvement to the home cage environment.

These data indicate that a short session, quasi-closed economy is suitable for assessing environmental interventions aimed at improving the welfare of laboratory rats. In terms of the second question about the necessity of reducing the weight of laboratory animals. The rats did show demand for condensed milk even when pellets were freely available. However the demand was less elastic when body weight was reduced to $85 \%$. Reducing the rats body weight probably does result in higher and more reliable response rates, but it may not be necessary for all experiments. 


\section{EXPERIMENT 4.2:}

\section{Rats' Demand for the Dimensions of Enrichment}

This experiment measured rats' demand for four dimensions of enrichment; space, rats, novel objects and cage fixtures. The same items from Experiment 3.2 were used. This allowed the preference and demand measures to be compared, and provided further information about the rats responses to these cage options.

In order to test demand for large environmental modifications the procedure used in Experiment 4.1 had to be modified somewhat. When the rats completed the FR requirement a door opened that allowed access to an adjoining cage that contained the cage modification being tested. After five minutes an alarm sounded and the experimenter placed the rats back into the working chamber. This procedure was similar to Sherwin \& Nicol's (1997) experiment on the demand of mice for larger cages.

A number of researchers have suggested that demand procedures are the best way to assess an animal's needs (Dawkins, 1992), and are a natural end-point to the use of preference (Matthews, 1993). However, very few researchers have gone on to make use of this technique in the field of welfare. Two researchers that did use demand procedures in the area of welfare were Sherwin and Nicol who studied demand for environmental options with mice (Sherwin, 1996; Sherwin \& Nicol, 1996, 1997). However their studies were limited in one of two ways, either they used a limited range of options, or used a procedure that cannot be analysed using Hursh's (1980) analysis. 
Sherwin and Nicol (1997) used only one commodity type (space). Preference is a relative measure, and demand as an extension of preference, shares that quality. The use of a single commodity would only be useful if demand procedures could produce absolute quantifications of demand, if demand measures are relative then other commodities must be provided as a basis for comparison. The relative nature of demand measures are, in fact, a greater difficulty with the demand procedure than was the case for preference. Preference has as its baseline the choices that would result from a random chance distribution, and deviations above or below this can be tested for statistical significance. With demand for a single commodity, no such statistical baseline exists. Also, Sherwin and Nicol (1997) used no prices above FR80, and fitted a straight line to the data. This limitation of the FR range might make the conditions seem more similar and more inelastic than they actually were.

Other studies by Sherwin and Nicol used multiple commodities, but are difficult to interpret for other reasons. Sherwin (1996) and Sherwin and Nicol (1996) used a procedure where the subjects could remain with the commodity until they chose to leave. This meant there was no unit price, in other words after the animal met the FR requirement it could consume as much of the commodity as it wished. This prevented the use of normal methods of interpreting demand including the use of the log slope of the demand function to measure elasticity. To be fair, these experiments were not intended to be classic demand experiments and they were not interpreted in this way. The results used were a balance of the number of entries into an environment and the amount of time spent before exiting again. Entries and dwelling times were treated as independent ways for the animal to respond to price increases. The other difficulty was the use of a 24 hour procedure. This meant that the dwelling times were greatly influenced by where the animal slept. The options provided across two 
experiments included many of the frequently used aspects of enrichment; a familiar mouse, extra space, objects, as well as a wheel, shelter and food. However in most cases significantly higher preferences were shown for only one option, the environment where the animal slept (the 'food' option in Experiment 1, and sawdust in Experiment 2, Sherwin \& Nicol, 1996). Other significant differences were rare and difficult to interpret. For example, the large cage was visited more often than the shelter. Given that the mice generally slept in the food bowl this is probably due to some problem with the shelter rather than reflecting the demand of mice for shelters. The shelter used was a plastic cup similar to the pottles not preferred by the rats in Experiment 3.2, rather than the more enclosed tins that were preferred.

In summary, those experiments that have used a demand procedure to assess cage modifications with rodents, were not able to take advantage of the theoretical basis of economic demand as originally formulated by Hursh (1984). If the full demand procedure is not practicable to use in welfare research, it would be best to investigate this now rather than simply let the theoretical and applied research gradually drift further apart.

\section{Method}

\section{$\underline{\text { Subjects }}$}

The subjects were six female Hooded Norway rats (two groups of three), that were 90 days old at the beginning of the experiment. They were kept under the standard conditions as described in Experiment 1. In the home cage the subjects had free access to complete diet pellets and water. 


\section{Apparatus}

One Commodore PC-10 computer used Pascal software to schedule experimental effects. The computer was connected by a custom built interface to a small response chamber containing one lever and one light. The response chamber was joined by a $20 \times 20 \mathrm{~cm}$ wide wooden tunnel to a cage containing the environment being used as the commodity. The tunnel was bisected by a cantilever door which opened when the FR requirement was met. At the end of the tunnel a pressure plate operated a switch which began the 5 minute timer and immediately re-closed the door to prevent access back to the response chamber.

\section{$\underline{\text { Procedure }}$}

The rats were trained using chocolate chips as reinforcers. They were trained to reliably lever press and traverse the tunnel, before the experiment began. The order of conditions was counter-balanced. Daily sessions lasted for 90 minutes. In each condition, completion of the fixed ratio lever pressing requirement opened the door and gave the rats access to the environment conditions presented in an otherwise standard cage. The FR began at a value of one for two sessions, and then increased by two (FR2 Group) or ten (FR10 Group) each session until a session occurred in which the FR requirement was not achieved (and therefore access to the commodity was not achieved). Next, demand for another commodity was assessed. Each subject was tested two (FR2 Group) or three (FR10 Group) times with each commodity. The commodities from Experiment 3.2 were used, these were: access to three familiar rats, novel objects, a double-sized cage and pillars. Three subjects were tested using FR2 steps, these subjects demand for chocolate was also assessed. For the other three subjects the FR incremented by 10 each session and their procedure included the full semi- 
enriched condition as a commodity. As a basis of comparison demand for access to a standard cage was also used as a baseline for both groups.

\section{Results}

\section{FR2 Group}

Figure 4.4 shows the parameters of demand curves generated by the three subjects when the FR requirement was incremented in steps of two. Equation 4.0 was not an adequate fit to the data for this group, in that it did not achieve convergence by 100 iterations with starting values of 1.0 (for $~ 60 \%$ of the data). When fits did converge (after extra iterations) they did not provide a good visual description of the data. For this reason this groups data was analysed using consumption rates at FR2 (rather than $L$ ) and last FR worked (instead of pmax). The individual parameters are shown in Appendix 4.2.

The consumption rates indicate that preference for all of the commodities was similar to, or less than that for the standard cage. Only access to chocolate, and possibly space, produced FR2 consumption rates similar to those for the standard cage.

The final FR value suggests that demand was most inelastic for access to the group of three rats. Table 4.2.1 shows the order of elasticity for the individual data from 1 (most inelastic) to 6 (most elastic). This confirms that access to the group of three rats resulted in the most inelastic data for all three subjects. No other commodity consistantly produced data that was more inelastic than that for the standard cage. Chocolate was included in this experiment 

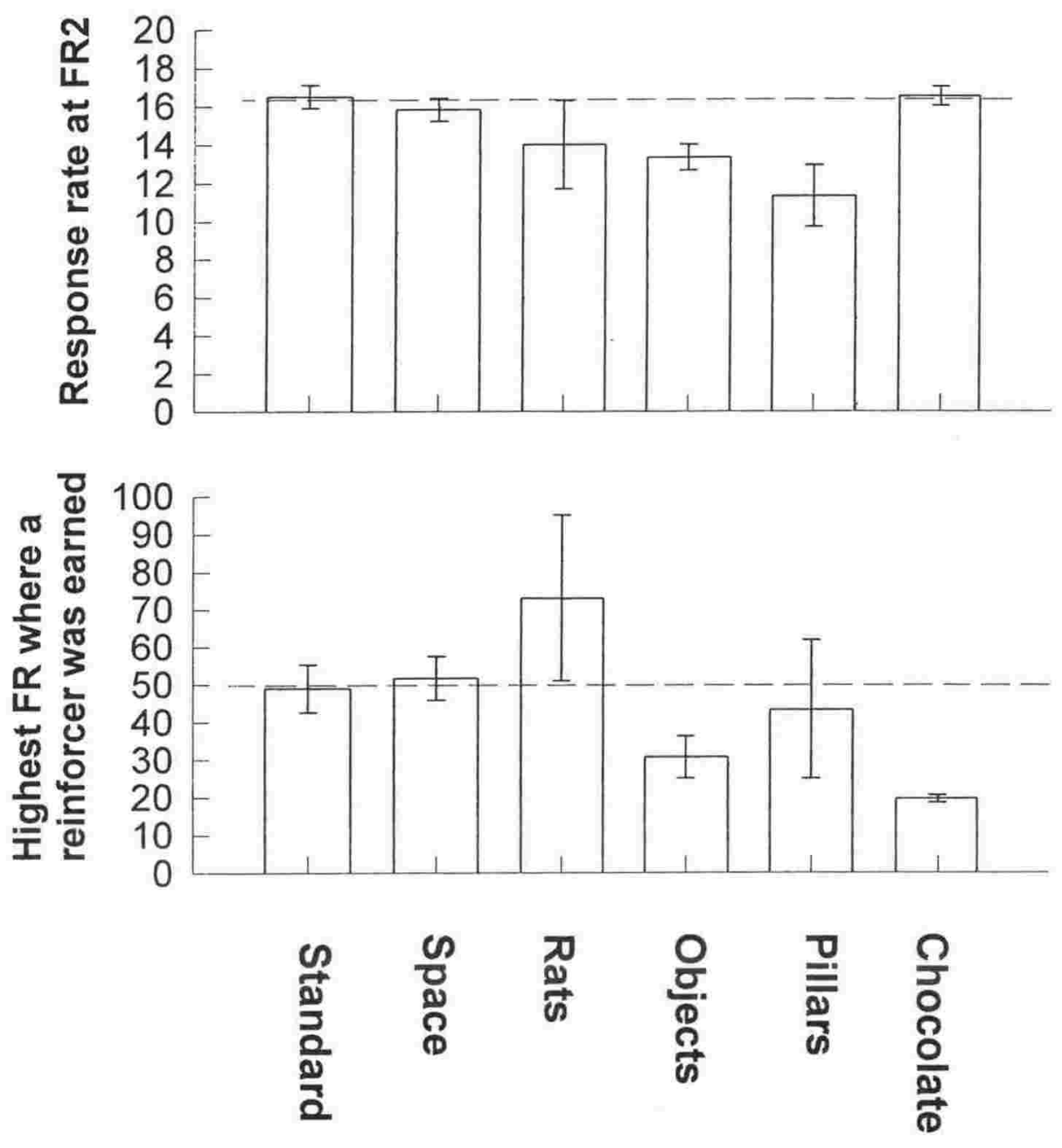

\section{Commodoties}

Figure 4.4 Intensity (response rate at FR2) and elasticity (last FR with at least one reinforcer earned) of demand for FR2 Group (FR2 steps). Arithmetic means and standard errors for three subjects. The dotted line indicates standard cage data. 
because it was thought that high demand would be shown for it, and this could be compared with the other commodities. Although chocolate produced some of the highest preferences, demand for its was consistently low. Thus it does not serve as a useful benchmark.

Table 4.2.1

Individual ratings of commodities based on individual's last FR where a commodity was obtained, bold type indicates median data that is more inelastic than that for a standard cage.

\begin{tabular}{|c|c|c|c|c|c|c|c|}
\hline rank & Subject 1 & $\underline{\text { rank }}$ & Subject 2 & rank & Subject 3 & rank & Group Mean \\
\hline 1 & 3 rats & 1 & 3 rats & 1 & 3 rats & 1 & 3 rats \\
\hline 2 & standard & 2 & space & 2 & pillars & 2 & space \\
\hline 3 & space & 3 & standard & 3 & standard & 3 & standard \\
\hline 4 & objects & 4 & pillars & 4 & space & 4 & pillars \\
\hline 5 & pillars & 4 & objects & 5 & objects & 5 & objects \\
\hline 6 & chocolate & 4 & chocolate & 6 & chocolate & 6 & chocolate \\
\hline
\end{tabular}

\section{FR10 Group}

With this group the FR steps were increased to ten. This allowed three lines to be generated rather than two, so the data are based on the median line of the three for each subject. These data was well described by Equation 4.0, so the $L$, a and $b$ parameters are used in the following section. Chocolate was no longer used as a commodity, the semi-enriched condition was now used as a commodity that might produce high demand.

Figure 4.5 shows the data for subjects when the FR requirement incremented by 10 . This figure used the mean of each subjects median data from the three repetitions with each commodity. That is, the median parameters of $L$, and pmax were based on the median $a$ and $b$ values for each subject (using Equation 4.1 and transforming the data from loge to log 10 ). 

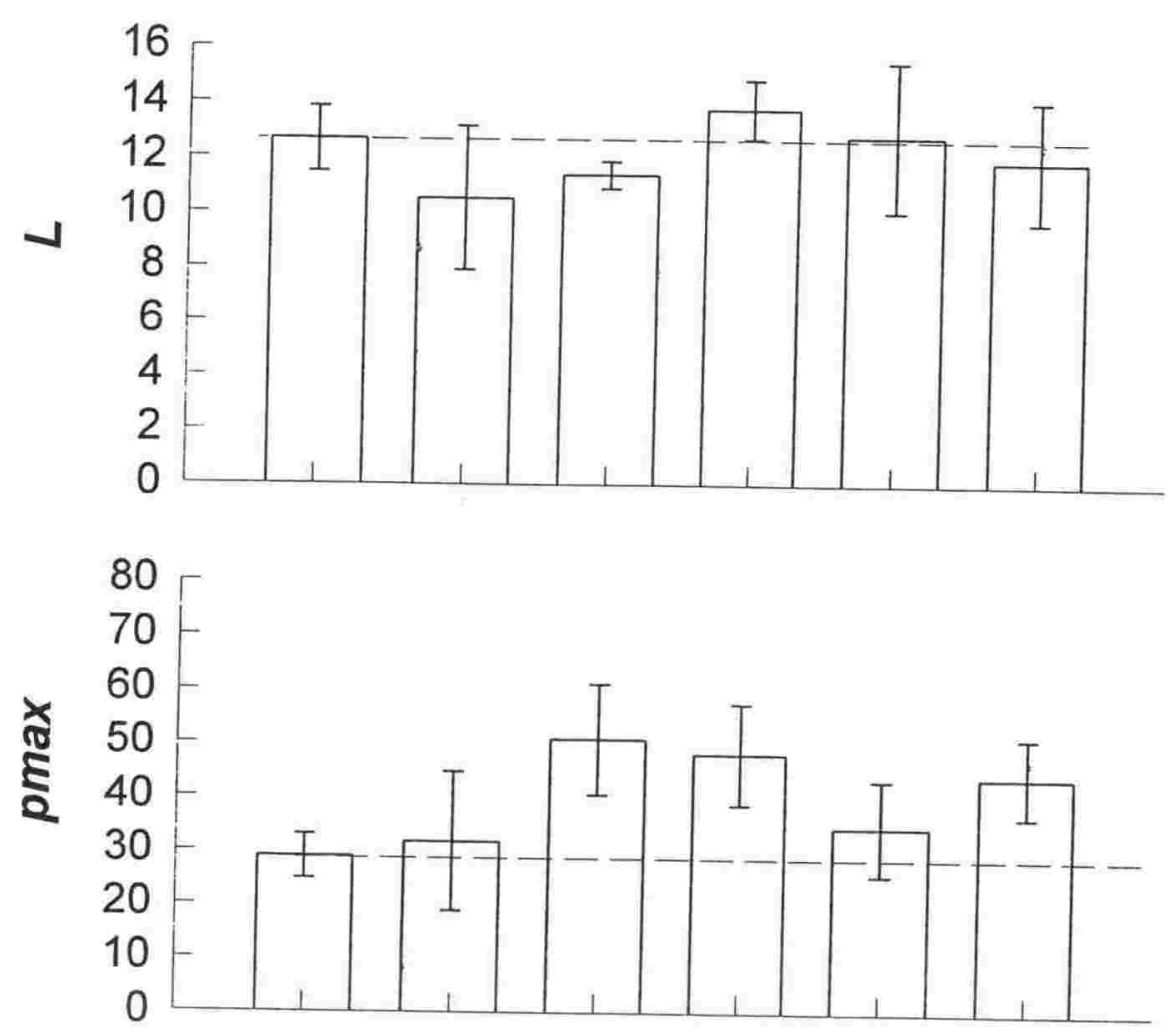

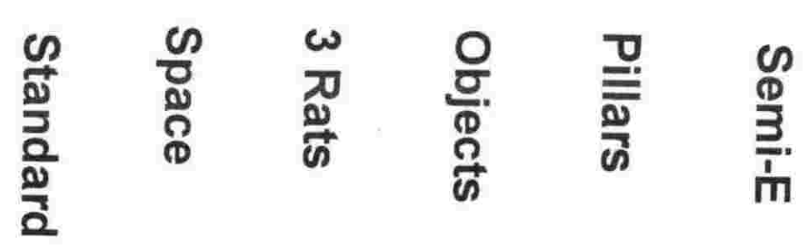

Commodities

Figure 4.5 Intensity $(L)$ and elasticity (pmax) of demand for FR10 Group (FR10 steps).

Arithmetic means and standard errors for three subjects. The dotted line shows data relating to the standard cage. 
The $L$ values for all of the commodities were similar to those for the standard cage, except that $L$ values for the group of three rats were somewhat lower. The three rats option produced lowest ranked $L$ values for two of the three subjects, but it was the second most preferred commodity for the third subject. The pmax values for the three rats, novel objects and semienriched caging were higher than for the standard cage.

Table 4.2.2 shows the commodities from most inelastic to least elastic for each subject. This shows that all three subjects showed demand for the semi-enriched condition that was higher than for the standard cage. For the other commodities Subjects 2 and 3 showed most inelastic demand for rats, followed by objects and space. However Subject 1 showed a different pattern of demand and only pillars had higher pmax values than the standard cage.

Table 4.2 .2

Individual ratings of commodities based on individual's pmax data. Bold type indicates median data that is more inelastic than that for a standard cage.

\begin{tabular}{|c|c|c|c|c|c|c|c|}
\hline$\underline{\text { rank }}$ & Subject 1 & $\underline{\text { rank }}$ & Subject 3 & $\underline{\text { rank }}$ & Subject 2 & rank & Group Mean \\
\hline 1 & semi-enriched & 1 & rats & 1 & rats & 1 & rats \\
\hline 2 & pillars & 2 & objects & 2 & objects & 2 & objects \\
\hline 3.5 & standard & 3 & space & 3 & semi-enriched & 3 & semi-enriched \\
\hline 3.5 & objects & 4 & semi-enriched & 4 & space & 4 & pillars \\
\hline 5 & rats & 5 & standard & 5 & standard & 5 & space \\
\hline 6 & space & 6 & pillars & 6 & pillars & 6 & standard \\
\hline
\end{tabular}

In summary, the two subject groups differed in size of FR increment between sessions. This had an effect on the fit of the line. The negatively accelerating line was an acceptable fit only with the FR10 steps and not with the FR2. In terms of overall levels of elasticity and 
intensity of demand, there were only small differences between the groups despite the use of different methods of quantification.

The finding common to both groups was that demand for access to the group of three rats, and the semi-enriched condition which also contained the three rats, tended to be the most inelastic. Intensity of demand at low FRs (reinforcers obtained at FR2 or $L$ ) tended to be at or below the level for the standard cage for all commodities.

\section{Discussion}

The $L$ parameter or consumption rate at FR2 did not clearly differentiate between the commodities. This preference related data failed to support the preference findings from Experiment 3.2, that the group of three rats and semi-enriched caging were significantly preferred over an empty cage, whilst the other commodities were not. Thus these measures were not equivalent to the preference tests used in Experiment 3. One factor which may have contributed to the difference between these measures of preference might be the novelty of the items. The preference measures in Experiment 3 were averaged over at least ten days of exposure to the items, whilst the $L$ parameter or FR2 response rates depended on only a few sessions at the beginning of the animals experience with the new commodity. The rats in the current experiment might show some avoidance of conditions that are dissimilar to the home cage, thus depressing their $L$ parameters or FR2 response rate for many of the commodities. Overall it seems that testing preference directly is a better option than relying on the use of parameters derived from a demand procedure. 
The pmax or last FR worked on, more clearly differentiated between the different cage options, however individual variation was quite high. Both subject groups produced a hierarchy that rated the group of three rats at the top, and rated the standard conditions at or near the bottom. Based on these results it could be said that demand for the company of a larger group of rats was the most inelastic, and thus this would be the best form of enrichment amongst the options investigated here. While the other options produced more modest or less reliable gains, they were all frequently favoured over the standard cage (as measured by demand elasticity).

The research reported here suggests that cage enrichment should concentrate upon social forms of enrichment. The following experiment provided environments that housed larger group sizes and assessed a subject's demand for these conditions. 


\section{EXPERIMENT 4.3:}

\section{Demand for Different Sized Social Groups}

Cage environments that include larger groups of rats probably need to be larger than standard cages. However, as the larger cage was not highly preferred or demanded the standard cage probably provides enough space for a pair of rats. Group size could be increased by joining together standard cages into larger units. This procedure has been previously suggested by Batchelor (1993).

The following experiment tested demand for cages that are double standard size with four rats (semi-enriched), and triple standard size with six rats (6-rat). Also included were the deprived and enriched conditions as comparisons. It was expected that the enriched condition would produce the most inelastic demand, and provide a basis of comparison for the other conditions (as in Experiment 1). However, the data also provides a test of whether the most enriched condition provided in Experiment 1, would in fact result in higher demand.

\section{Method}

\section{$\underline{\text { Subjects }}$}

The subjects were four female Hooded Norway rats that were one year old at the beginning of the experiment. They were housed under standard conditions as described in Experiment 1. 


\section{Apparatus}

A demand apparatus was constructed that was very similar to that used in Experiment 4.2. It differed in that the door had two panels that were lifted apart from above rather than levered up. This made the door more difficult for the rats to move when it was meant to be closed, thereby reducing the supervision that the procedure required. Also the pressure plate that signalled the animal had passed through the tunnel was replaced with the hinged door. This door had a right angle bend and one way swing that made it difficult to open from the outside.

\section{Procedure}

The procedure followed that from the previous experiment with the following exceptions. There was a single group which comprised four subjects, and the FR requirement was incremented in steps of 10 between sessions. The conditions used were access to group sizes of zero, one, three, five and eleven other rats. The corresponding cage sizes were; the deprived cage, a standard cage, a semi-enriched cage, a cage constructed from three standard cages and the enriched cage (as described in Experiment 1). This provided a floor area of $20 \times 20 \mathrm{~cm}$ for each rat in the group including the subject. In all but the enriched (12 rat) condition the rats used were housed interchangeably with the subject. This involved moving the rats between cages so that they met each other group member within the week. Chocolate was included as commodity of a different type, to provide a wider basis of comparison. 


\section{Results}

The data and parameters for Equation 4.0 fitted to these data are shown in Appendix 4.3.

Figure 4.6 shows the overall mean $L$ and pmax values for each commodity. A median was taken of each rats demand for each commodity (across the three trials) and a mean taken of the four subjects median data, to produce the group average. The $L$ and pmax values were similar in magnitude to the previous experiment. The highest initial preference ( $L$ value) was shown for the standard conditions. The lowest initial preference was for the deprived conditions.

If the standard ( 2 rat) condition was used as a baseline the demand for the deprived ( 1 rat) condition was more elastic, and demand for the 6 rat condition was more inelastic. The other three conditions (semi-enriched, enriched and chocolate) have elasticity of demand roughly equivalent to that of the standard cage. Table 4.3 shows the elasticity hierarchies of the individual subjects (with the most inelastic demand ranked 1st). This shows that the individual data is actually very variable. There is a slight tendency for the 6 rats data to be more inelastic than that for other conditions (ranked 1-3 for all of the subjects and above the standard 2 rat condition in the group averages). As in Experiment 4.2, demand for chocolate was fairly elastic. 

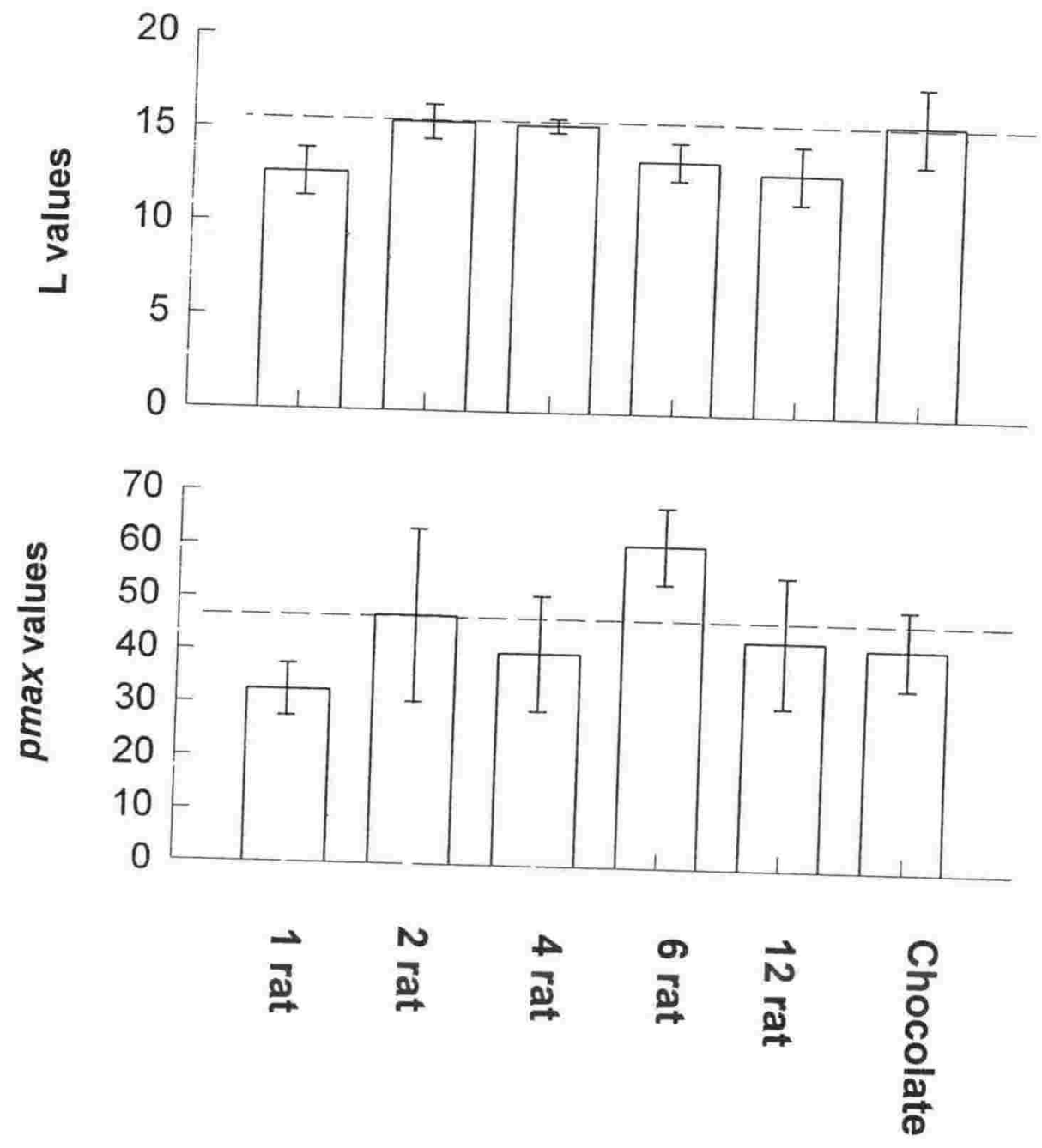

Commodities

Figure 4.6 Intensity $(L)$ and elasticity (pmax) of demand for six commodities (FR10 steps).

Arithmetic means and standard errors for four subjects. The dotted line shows data relating to the standard cage. 


\section{Table 4.3}

Inelasticity hierarchies based on pmax parameters, with data more inelastic than that for the standard cage shown in bold type.

\begin{tabular}{|c|c|c|c|c|c|}
\hline & Subject 1 & Subject 2 & Subject 3 & Subject 4 & Group Mean \\
\hline $1 \mathrm{st}$ & 4 rat & 12 rat & 2 rat & 6 rat & 6 rat \\
\hline $2 \mathrm{nd}$ & 1 rat & 6-rat & 6 rat & 1 rat & 2 rat \\
\hline 3rd & 6 rat & 4 rat & 4 rat & 2 rat & 12 rat \\
\hline 4th & 2 rat & chocolate & chocolate & chocolate & chocolate \\
\hline 5 th & 12 rat & 2 rat & 12 rat & 12 rat & 4 rat \\
\hline 6 th & chocolate & 1 rat & 1 rat & 4 rat & 1 rat \\
\hline
\end{tabular}

\section{Discussion}

The $L$ parameters were not particularly informative and did not appear to vary consistently with group size. There was some evidence that the rats favoured the familiar standard cage in that this condition was initially most preferred (i.e. higher $L$ values). So it may be that those conditions most different from the standard conditions will be rejected (both deprived and enriched). Traditional preference tests (as in Experiment 3) seem to differentiate more clearly between conditions. This might be partially because the preference procedure allows the experimenter to pre-expose the subjects to each condition thus reducing the confounding effects of novelty.

The overall conclusion from Chapter 4 is that laboratory rats should be kept in social groups, and that this can be accomplished without necessarily providing more space per rats. However, there was not a strong tendency to demand one group size over another. The group size that produced somewhat consistently highly elastic data was the 6 rat condition, however more data would be useful to confirm this suggestion. 
Elasticity of demand for different group sizes varied greatly between individuals. This partly reflects the variable nature of the demand data in general, compounded by the limited amount of data that can be collected using a semi-automated procedure. However, it also may reflect that the rats as individuals do differ in their preferences. Qualitative observations seemed to suggest that socially dominant rats (such as Subject 2) had a higher demand for larger groups of rats, while subordinates were equally likely to choose smaller groups. It is also possible that the relative familiarity of the environment is disrupting the data. This is a factor that could be examined in future studies that assess social dominance and other social relationships in the group, and using subjects raised in a range of housing conditions.

There was also a confounding factor with the fully enriched condition in that rats from the semi-enriched and 6-rat conditions were familiar to the subjects. In the enriched condition only six of the rats were familiar from the beginning of the session. The other five dwelt continually in the enriched cage and were encountered only during the session. It was considered impossible to house all 12 rats interchangeably and maintain a reasonable level of familiarity. Also it was apparent that when the subjects entered the enriched cage they were often 'mobbed' by the other rats and/or pushed aside by rats trying (usually unsuccessfully) to get through the tunnel before the door closed. These behaviours may have made entering the enriched condition less rewarding. These patterns were not observed in the semi-enriched or 6-rat cages.

Another option for clarifying the issue of optimal group size would be to return to the preference tests of Chapter 3. This could provide independent supporting evidence of the trends identified using this demand procedure. While the data provided by demand 
procedures is more multi-dimensional than that from enrichment, it is also less widely used and so further work needs to be done before demand results can be used in this area with a high degree of confidence. However, once the details of the procedure are decided upon, demand should provide the best possible information about the reinforcing value of environmental enrichment options, adding a whole extra dimension to the kinds of information extracted from preference procedures.

In terms of the procedural difficulties that require further scrutiny, the following were most obvious during the course of data collection. Data collection was time consuming due to the session time occupied by consumption periods and the manual procedure, and this severely limited the number of subjects that could be run. Increasing the subject numbers might produce more reliable measures of central tendency. One possibility might be running a large number of demand chambers in tandem.

The other problem was that both subjects and non-subjects 'enrichment' rats attempted to manually force the doors open. This behaviour was fairly persistent, it increased wear and tear on the equipment and meant the procedure needed to be fairly closely monitored. However coping with these difficulties was better than using restrained enrichment rats and thus restricting the social contact possible between the subject and enrichment rats. 


\section{General Discussion}

\section{Emergence, Open Field Activity and Hebb William's Maze Errors (Chapter 1)}

\section{The Findings}

If the validity of the traditional methods of assessing environmental enrichment are accepted, then concerns arise regarding the status of the standard laboratory rat. Experiment 1 demonstrated that the standard rat's data in the emergence box, Hebb William's maze and open field resembles that of the deprived rats more closely than that of the enriched rat. If the deprived rat is considered to have compromised welfare (compared with the enriched rat) then it follows that rats housed in standard laboratory conditions also suffer compromised welfare.

\section{The Procedures and Suggestions for Further Research}

There is no reason to doubt the validity of the emergence or field activity data as a measure of fear or neophobia. The enriched rats have more opportunities to habituate to the harmful potential of novel stimuli, whilst the deprived rats show the normal inherited pattern of fearful reaction to unknown objects or situations (as shown by wild rats, Boice, 1977; Cowan, 1977). In some sense this is a direct transfer effect as the testing enrionments are more different from the deprived cage than the enriched cage. However, I do not see this a flaw, as the source of the deprived rats fearfulness is its restricted experience and subsequent stressfulness of adjustment to more stimulating environments. Presumably, if the deprived rat never leaves its cage then fearfulness is not an issue, but inevitably any rat will be handled and removed from its secure environment, and this is when a fearfulness problem ensues. It would be useful to determine the generality of deprivation-induced neophobia. The open 
field uses a large open space, but it is not clear whether similar responses would occur in the presence of unfamiliar rats, fixtures, sounds, objects or other stimuli. The rats could be tested for freezing in the presence of these different stimuli. This would help expose the extent to which open field results reflected direct transfer and how much is due to a more general phenomenon like fearfulness or emotionality. In the absence of such evidence the direct transfer explanation is more parsimonious.

The basic validity of the open field procedure is often under-mined by poor standardisation. One of the most problematic is the variation in session length and the use of individual scores that are mean or sum data for long or multiple sessions. During long sessions the rate of habituation to the novelty of the situation represents a significant confounding variable, especially as this rate varies systematically as a result of housing condition (Experiment 1). Open field data should be routinely investigated for time based trends, and if these exist, whole session averaging should not be used. However if one recognises that the group differences in time course of activity have a significance in their own right, this moves it from being a confounding variable to an enlightening and valid dependent variable with its own implications for welfare. The levels of the freezing response need to be balanced by an understanding of their duration and persistence. Averaging these factors diminishes the deprived rats apparent emotionality. Treating them separately shows more clearly that the deprived rats display more initial freezing, and greater persistence of freezing throughout the session.

The Hebb William's maze procedure is not a valid test of environmental effects on cognition. It seems probable that it is not a valid cognitive test in any context. Researchers examining 
cognitive and neophobic effects of housing seem to be to willing to ascribe results to higher order behaviours like learning or emotionality when more simple phenomenon would produce identical results. The deprived rats greater sensitivity to stimulation is a problematic confound in the Hebb William's maze and other tests that are intended to assess cognitive abilities. This sensitivity may produce a neophobic response under some situations or greater sensitivity to some source of reinforcement in others. In the open mazes like the Hebb William's it may be that the deprived rats are more sensitive to the reinforcement available for exploring the environment rather than moving through it quickly to get to the food.

\section{Tests of Specific Cognitive Abilities (Chapter 2)}

\section{The Findings}

Independent verification of a global cognitive deficit caused by environmental deprivation, could not be obtained in the areas of learning, memory or persistence. However, attention or encoding of discriminations under conditions of disruption do tend to differ between groups. Thus it is suggested that studies that have not used electrical shock, water or open spaces have still incorporated a more subtle disadvantage to the deprived rats. They all incorporate extinction, normally in the form of visual discrimination reversal (Bingham \& Griffiths, 1952, etc.). This sudden extinguishing of continuous reinforcement might produce a classic 'frustration' response in the more sensitive deprived rats, in that they persistently respond to the previously correct position before eventually learning the currently correct behaviour (Experiment 2.3). The unimpaired cognitive abilities of the rats are demonstrated by their equality with the enriched rats performance in initial learning and superiority in reversal tasks using partial rather than continuous reinforcement. It would be unparsimonious to suggest that the deprived rats intelligence is some-how context specific. Rather it is that their 
cognitive abilities are sometimes concealed by their heightened sensitivity to the intrusion of any change or novelty in a familiar situation. This sensitivity is not always a disadvantage as the deprived rats demonstrate an enhanced sensitivity to reinforcement which is probably responsible for their superior performance in VI based learning tasks.

\section{Conclusions From Chapters 1 and 2}

Standard rats differ from enriched rats in two main ways. One, a tendency to be more fearful (Experiment 1). Two, a heightened reactivity to a range of events including food reinforcement (Experiments 2.3 and 2.4), some forms of disruption (Experiment 2.1) and extinction (Experiment 2.2).

The fearfulness of the environmentally deprived rat might be mitigated by enrichment, but other treatments might also be effective, for example human handling. Human handling might be sufficient to normalise excessive neophobic reactions (Spence \& Maher, 1962). Furthermore, many behavioural experiments may provide appropriate extra-cage enrichment for rats which would normally spend much of their time foraging for food. Many behavioural procedures are foraging tasks of some complexity and might compensate to some extent for aspects that the home environment lacks. Thus the data so far suggest that the standard conditions are not necessarily unacceptable conditions for adult animals.

The effect of housing on sensitivity to reinforcement, disruption and extinction should be kept in mind when generalising from specific data, as rats in one housing condition may not be representative of the capacities of the species as a whole. Also housing should be kept uniform within an experiment to prevent it acting as a confounding variable. The deprived 
rats behaviours may indicate a lack of environmental stimulation in the home cage, which should be corrected or provided from other sources. However, these behaviours are not in themselves deficits, as some (sensitivity to reinforcement) can function as advantages in some experiments.

\section{Preference (Chapter 3)}

\section{The Findings}

While standard caging may not always produce marked behavioural deficits, rats housed under these conditions do show significant preferences for some forms of enrichment. The use of preference procedures changed the emphasis of the thesis from one of damage control to one of pro-active concern for the animals quality of life. Preference data supports the provision of nesting boxes, paper and platforms as the best forms of cage furniture (Experiments 3.2 and 3.3). Preference was also shown for enrichment and particularly for the larger group size of three familiar rats (Experiment 3.1). There may be other environments that the rats would prefer, but these were not tested here. In fact, the literature in general would benefit from a greater data-base of preference results, using a wide variety of caging options.

\section{The Procedures}

Both T-maze and continuous access seem to be a useful methods of assessing interventions like cage furniture. The T-maze seems to give higher estimates of rats' preferences for 'activity' substrates, while the continuous access box favours 'resting' substrates. For this reason these two procedures should probably be used in tandem rather than separately. 


\section{Demand (Chapter 4)}

\section{The Findings}

Demand data were a little more difficult to interpret than the preference data. The findings show that even rats that are regularly handled by humans, display relatively inelastic demand for contact with other rats (Chapter 4). As such, rats should be socially housed, and there is some evidence that group sizes of about six rats would be advisable (Experiment 4.3). This need not require any more space as the ratio of space per rat remained constant across all conditions.

\section{The Procedure}

The main difficulty with demand is that when large commodities are used it was a time consuming procedure, and this restricted the number of subjects used and sessions completed. The data in Chapter 4 represents two years of data collection, averaging 4.5 hour per day, seven days a week. The limited number of subjects made the data set smaller and less reliable compared with, for example, preference procedures. However, the elasticity findings were broadly consistent with the preference data from Chapter 3 , particularly as both favoured larger group sizes as the commodity the rats were most motivated to enter. It might be a mistake to depend on elasticity measures exclusively as it should probably augment preference data rather than replace it. The problems with the demand procedure are practical matters involving the work and time involved and the many possible confounding variables involved with the more complex procedure. However the greater validity of the demand procedures assessment of the environments reinforcing value makes addressing these difficulties worthwhile. 


\section{Conclusion}

Based on the current findings I would advise the immediate provision of nesting material to rats under standard conditions, and the purchase of cages suitable for larger groups when this is practicable. I would suggest the provision of novel items, platforms or pillars when this is convenient. I would advise against the provision of cage furniture of the types that were not preferred, such as tunnels or sticks.

There are many aspects of this thesis which suggest areas of further research. The criticism of the Hebb William's maze undermines much that was learned using this procedure, but the maze has been largely replaced in most areas (often by operant techniques such as those used in Chapter 2). In fact, the deprived rats lack of wide-spread cognitive deficits will probably come as no surprise to many researchers despite the weight of evidence to the contrary (Chapter 1, Section 1.2). However, it is a finding that would benefit from independent replication.

On the other hand, the open field is still seeing a great deal of use (for example, Cheal \& Foley, 1985; van de Weerd et. al., 1994). Efforts to investigate the confounding variables (light, session length etc.) and to increase the standardisation of this task would be a great service to those trying to understand the data it produces.

The suggestion that deprived rats have an advantage in some learning tasks because they are more sensitive to the available reinforcement, with a specific disadvantage in that they respond more frequently under extinction is a more adventurous suggestion. The suggestion could be validated by testing enriched and deprived rats on other learning tasks that do not 
incorporate extinction. The most obvious example would be errorless discrimination learning. It would be expected that deprived rats will perform better than enriched rats on this task. This is because deprived rats would not be disadvantaged by their enhanced frustration, and would benefit from their greater sensitivity to reinforcement (Experiment 2.4).

The same is true, to some extent with demand procedures. If a larger number of studies existed then informative consistencies might emerge and the data could be applied with greater confidence.

To summarise, differential housing conditions produced behavioural differences in rats.

Overall, these difference indicate that the welfare of rats in standard housing conditions could be improved. Nesting material and social contact should be provided or extended for most laboratory rats. Further research is indicated in order to better understand the effect of environmental enrichment and methods for effectively enriching cage environments. The data that exists in this and other studies is beginning to support an understanding of how to protect the welfare of captive species, but the current state of animal welfare research techniques and findings does allow for any complacency. 


\section{References}

Ader, R. (1965). Effects of early experience and differential housing on behavior and susceptibility to gastric erosions in the rat. Journal of Comparative and Physiological Psychology, 60(2), 233-238**

Appleby, M. C. (1997). Life in a variable world: behaviour, welfare and environmental design. Applied Animal Behaviour Science, 54, 1-19.

Anzaldo, A. J., Harrison, P. C., Riskowski, G. L., Maghirangi, R-G and Gonyou, H. W. (1994). Increasing welfare of laboratory rats with the help of spatially enhanced cages. Animal Welfare Information Centre Newsletters, 5(3), 1-5.

Archer, J. (1973).Tests for emotionality in rats and mice: a review. Animal Behavior, $21,205-235$.

Bantin, G. C. \& Sanders, P. D. (1989). Animal caging: is bigger necessarily better? Animal Technology, 40(1), 45-54.

Barnett, (1963). A Study in Behaviour: Principles of Ethology and Behavioural Physiology Displayed mainly in the Rat. London: Methuen Co. Ltd.

Batchelor, G. R. (1993). An enriched commune housing system for laboratory rats - a preliminary view. Animal Technology, 44 (3), 201-213.

Baum, W. M. (1974). On two types of deviation from the matching law: bias and undermatching. Journal of the Experimental Analysis of Behavior, 22, 231-242.

Bennett, E. L., Diamond, M. L., Krech, D. \& Rosenzweig, M. P. (1964). Chemical and anatomical plasticity of brain. Science, 146, 610-619. 
Bingham W. E. \& Griffiths, W. J. (1952). The effect of differential environments during infancy on adult behavior in the rat. Journal of Comparative and Physiological Psychology, 45, 307-312.*

Blanchard, R. J., Kelly, M. J. \& Blanchard, D. C. (1974). Defensive reactions and exploratory behavior in rats. Journal of Comparative and Physiological Psychology, 87(6), 1129-1133.

Blom, H. J. M., Baumans, V., Van Vorstenbosch, C. J. A. H. V. \& Beynen, A. C. (1993). Preference test with rodents to assess housing conditions. Animal Welfare, 2, 67-80.

Blough, D. S. (1959). Delayed Matching in the Pigeon, Journal of Experimental Analysis of Behaviour, 151-160.

Boice, R. (1977). Burrows of wild and albino rats: effects of domestication, outdoor raising, age, experience, and maternal state. Journal of Comparative and Physiological Psychology, 91(3), 649-661.

Bradshaw, A. L. \& Poling, A. (1991). Choice by rats for enriched versus standard home cages: plastic pipes, wood platforms, wood chips, and paper towels as enrichment items. Journal of the Experimental Analysis of Behavior, 55, 245-250.

Broadhurst, P. L. (1956). Determinants of emotionality in the rat II: antecedent factors. British Journal of Psychology, 49, 12-20.

Brown, R. T. (1968). Early experience and problem-solving ability. Journal of Comparative and Physiological Psychology, 65(3), 433-440.*

Brown, P. S., Down, M. L. \& Wyatt, A. C. (1968). Caging rats for a bioassay. Journal of the Institute of Animal Technicians, 19 (2) 78-84. 
Candland, D. K., Horoquitz, S. H. \& Culbertson, J. L. (1962). Acquisition and retention of acquired avoidance with gentling as reinforcement. Journal of Comparative and Physiological Psychology, 55(6), 1062-1064.

Candland, D. K. \& Nagy, Z. M. (1969). The open field, some comparative data. Annals of the New York Academy of Science, 159, 46-51.

Chamove, A. S. (1984). Analysis of learning in retarded monkeys. Journal of Mental Deficiency Research, 28, 21-41.

Chamove, A. S. (1989). Cage design reduces emotionality in mice. Laboratory Animals, 23, 215-219.

Chamove, A. S. (1994). Enrichment - past and future. ANZCCART News, 7 (1), 4-5.

Coburn, J. F. \& Tarte, R. D. (1976). The effect of rearing environment on the contrafreeloading phenomenon in rats. Journal of the Experimental Analysis of Behaviour, 26, 289-294.

Cowan, P. E. (1977). Neophobia and neophilia: new-object and new-place reactions of the three rattus species, Journal of Physiological and Comparative Psychology, 91 (1), 6371.

Davenport, J. W., Gonzalez, L. M., Carey, J. C., Bishop, S. B. \& Hagquist, W. W. (1975). Environmental stimulation reduces learning deficits in experimental cretinism. Science, 191, 578-579*

Davis, H. \& Perusse, R. (1983). Human-based social interaction can reward a rat's behaviour. Animal Learning and Behavior, 16(1), 89-92.

Davison, M \& McCarthy, D. (1988). The Matching Law: a Research Review. New Jersey: Lawrence Erlbaum Asociates. 
Dawkins, M. (1976). Towards an objective method of assessing welfare in domestic fowl. Applied Animal Ethology, 2, 245-254.

Dawkins, M. (1977). Do hens suffer in battery cages? Environmental preference and welfare. Animal Behaviour, 25, 1034-1046.

Dawkins, M. (1978). Welfare and the structure of a battery cage: size and cage floor preferences in domestic hens. British Veterinary Journal, 134, 469-475.

Dawkins, M. S. (1990). From an animal's point of view: motivation, fitness, and animal welfare. Behavioural Brain Sciences, 13, 1-61.

Dawkins, M. S. (1992) Suffering, demand curves and welfare: a reply to Houston. Animal Behaviour, 53(3), 1119-1121.

Denenberg, V. H. \& Morton, J. R. C. (1962). Effects of environmental complexity and social groupings on modification of emotional behavior. Journal of Physiological and Comparative Psychology, 55(2), 242-246.*

Denenberg, V. H., Woodcock, J. M. \& Rosenberg, K. M. (1968). Long-term effects of preweaning and postweaning free-environment experience on rats' problem-solving behavior. Journal of Physiological and Comparative Psychology, 66(2), 533-535.*

Denny, M. S. (1975). The rat's long-term preference for complexity in its environment. Animal Learning and Behavior, 3(3), 245-249.

Doty, B. A. \& Doty, L. A. (1967). Effects of handling at various ages on later openfield behaviour. Canadian Journal of Psychology, 21 (6), 463-470.

Duncan, I. J. H. (1978). The interpretation of preference tests in animal behaviour. Applied Animal Behavior, 4, 197-200.

Einon, D. \& Morgan, M. (1976). Habituation of object contact in socially-reared and isolated rats (rattus norvegicus). Animal Behaviour, 24, 415-420.* 
English, J. A. R., Rawlet, J. K. \& Woolverton, W. L. (1995). Unit price analysis of opiod consumption by monkeys responding under a progressive ratio schedule of drug injection. Journal of the Experimental Analysis of Behavior, 64, 361-371.

F.A.W.C. (1992). The Five Freedoms. Farm Animal Welfare Council Press Release, London. cited in Mellor D.J. \& Reid, C.S.W. (1994). Assessment of Well-being. In Baker, R.M., Jenkin, G \& Mellor, D. J. Improving the Well-Being of Animals in the Research Environment (pp.3-18). Sydney: ANZCCART.

Forgays, D. G. \& Forgays, J. W. (1952). The nature of the effect of freeenvironmental experience in the rat. Journal of Physiological and Comparative Psychology, $45,322-328$.

Forgays, D. G. \& Read, J. M. (1962). Crucial periods for free-environmental experience in the rat. Journal of Physiological and Comparative Psychology, 55(5), 816818.*

Forgus, R. H. (1958). The effect of different kinds of form pre-exposure on form discrimination learning. Journal of Comparative and Physiological Psychology, 51, 75-78.

Foster, T. M., Blackman, K. A. \& Temple, W. (1997). Open versus closed economies: performance under fixed-ration schedules. Journal of the Experimental Analysis of Behavior, 67, 67-89.

Fraser, D., Weary, D. M., Pajor, E. A. \& Milligan, B. N. (1997). A scientific conception of animal welfare that reflects ethical concerns. Animal Welfare, 6(3), 187-205.

Gibson, E. J. \& Walk, R. D. (1956). The effect of prolonged exposure to visually presented patterns on learning to discriminate them. Journal of Comparative and Physiological Psychology, 49, 239-242. 
Gibson, E. J., Walk, R. D. \& Tighe, T. J. (1959). Enhancement and deprivation of visual stimulation during rearing as factors in visual discrimination learning. Journal of Comparative and Physiological Psychology, 52, 74-81.

Gill, J. H., Reid, L. D. \& Porter, P. B. (1966). Effects of restricted rearing on Lashley stand performance. Psychological Reports, 19, 239-242.*

Hall, F. S., Humbly, T., Wilkinson, L. S. \& Robbins, T. W. (1997). The effects of isolation rearing on preference by rats for a novel environment. Physiology and Behavior, 62 (2), 299-307.

Hebb, D. O. (1947). The effects of early experience on problem-solving at maturity. The American Psychologist, 2, 306-307.

Henderson, N. D. (1966a). Effects of intensity and spacing of prior stimulation on later emotional behavior. Journal of Comparative and Physiological Psychology, 62 (3), 441448*.

Henderson, N. D. (1966b). Changes in open field behaviour as a result of experimenter manipluation before or after intense shock. Canadian Journal of Psychology, 20 (3), 296-301.

Herrnstein, R. J. (1970). On the law of effect. Journal of the Applied Analysis of Behavior, 13, 243-266.

Heyman, G. M. (1983). A parametric evaluation of the hedonic and motoric effects of drugs; pimozide and amphetamine, Journal of the Experimental Analysis of Behavior, 40, 113-122.

Hogan, J. A., Kliest, S. \& Hutchings, C. S. L. (1970). Display and food as reinforcers in the siamese fighting fish (betta splendens), Journal of Comparative and Physiological Psychology, 70 (3), 351-357. 
Holson, R. R. (1986). Feeding neophobia: a possible explanation for the differential maze perfromance of rats reared in enriched or isolated environments. Physiology and Behavior, 38, 191-201*.

Huck, W. U. \& Price, E. O. (1975). Differential effects of environmental enrichment on the open-field behavior of wild and domestic Norway rats, Journal of Comparative and Physiological Psychology, 89(8), 802-808.

Hughes, K. R. (1965). Dorsal and ventral hippocampus lesions and maze learning: influence of pre-operative environment. Canadian Journal of Psychology, 19(4), 325-332.* Hughes, B. O. (1975). Spatial preference in the domestic hen. British Veterinary Journal, 131, 560-563.

Hughes, B. O. (1976) Preference decisions of domestic hens for wire of litter floors. Applied Animal Ethology, 2, 155-165.

Hughes, B. O. \& Black, A. J. (1973). The preference of domestic hens for different types of battery cage floor. British Poultry Science, 14, 615-619.

Hursh, S. R. (1980). Economic concepts for the analysis of behavior. Journal of the Experimental Analysis of Behavior, 34, 219-238.

Hursh, S. R. (1984). Behavioral Economics. Journal of the Experimental Analysis of Behavior, 42, 435-452.

Hursh, S. R. (1991). Behavioural economics of drug self-administration and drug abuse policy, Journal of the Experimental Analysis of Behavior, 56(2), 377-393.

Hursh, S. P., Raslear, T. G., Shurtleff, D., Bauman, R. \& Simmons (1988). A cost benefit analysis of demand for food. Journal of the Experimental Analysis of Behavior, 80(3), $419-440$ 
Hymovich, B. (1952). The effects of experimental variations on problem solving in the rat. Journal of Physiological and Comparative Psychology, 45, 313-321.*

Jones, G. H., Marsden, C. A. \& Robbins, T. W. (1990). Increased sensitivity to amphetamine and reward-related stimuli following social isolation in rats: possible disruption of dopamine-dependent mechanisms of the nucleus accumbens. Psychopharmacology (Berlin), 102, 364-372.

King, D. L. (1970) Effect of early experience and litter on some emotionality variables in the rat. Journal of Comparative and Physiological Psychology, 73, 436-441.

Kling, J. W. \& Riggs, L. A. (1972). Woodworth and Schlosberg's Experimental Psychology. Methuen \& Co. Ltd.: London.

Kolb, B. \& Elliott W. (1987). Recovery from early cortical damage in rats. II. Effects of experience on anatomy and behavior following frontal lesions at 1 or 5 days of age. Behavioural Brain Research, 26, 47-56.*

Krech, D., Rosenzweig, M. R. \& Bennett, E. L. (1962). Relations between brain chemistry and problem-solving among rats raised in enriched and impoverished environments. . Journal of Comparative and Physiological Psychology, 55(5), 801-807.*

Krech, D, Rosenzweig \& Bennett, E. L. (1966). Environmental impoverishment, social isolation and changes in brain chemistry and anatomy. Physiology and Behavior, 1, 99104.

Lavalee, R. J. (1970). Effects of visual form and distance experience before maturity and adult problem solving ability in the rat. Developmental Psychology, 2 (2), 257-263.

Lawlor, M (1990). The size of rodent cages. In Guttman (Ed.) Guidelines for the Wellbeing of Rodents in Research. (pp. 19-28). Bethesda: Scientist Centre for Animal Welfare. 
Lea, S. E. G. (1978). The psychology and economics of demand. Psychological Bulletin, 85(3), 411-466.

Lea, S. E. G. \& Roper, T. J. (1977). Demand for food on fixed-ratio schedules as a function of the quality of concurrently available reinforcement. Journal of the Experimental Analysis of Behavior, 27, 371-380.

Luchins, A. S. \& Forgus, R. H. (1955). The effect of differential post-weaning environment on rigidity of an animal behavior. The Journal of Genetic Psychology, 86, 51$58 .^{*}$

McCall, R. B., Lester, M. L. (1969). Differential rearing and the exploration of stimuli in the open field. Developmental Psychology, 1(6), 750-762.*

Macintosh, L. N J. (962). The effects of over-training on a reversal and a nonreversal shift. Journal of Comparative and Physiologocal psychology, 55(4), 555-559.

Matthews, L. R. (1994). A new methodology for assessing the behavioural requirements of captive animals. Improving the Well-Being of Animals in the Research Environment, Proceedings of a Conference held at the Marriott Hotel, Sydney, October, 1993, 143-146.

Mazur, J. E. (1998). Learning and Behaviour (4th ed.) Prentice Hall: New Jersey.

Meier, G. W. \& McGee, R. K. (1959). A re-evaluation of the effect of early perceptual experience on discrimination performance during adulthood. Journal of Comparative and Physiological Psychology, 52, 390-395.

Morgan, M. J. (1973). Effects of post-weaning environment on learning in the rat. Animal Behavior, 21, 429-442.*

Mundy, L. A. \& Porter, G (1969). Some effects of the physical environment on rats. Journal of the Institute of Animal Technicians, 20(2), 78-81 
Nevin, J. A. (1974). Response strength in multiple schedules. Journal of the Experimental Analysis of Behaviour, 21, 389-408.

Nevin, J. A. (1992). An integrative model for the study of behavioral momentum. Journal of the Experimental Analysis of Behaviour, 57, 301-316.

Nevin, J. A., Mandell, C. \& Atak, J. R. (1983). The analysis of behavioral momentum. Journal of the Experimental Analysis of Behaviour, 39, 49-59.

Nickolotsea, M. \& Lore, R. (1981). Aggression in domesticated rats reared in a burrow-digging environment. Aggressive Behavior, 7, 245-252.

Ough, B. R., Beatty, W. W. \& Khalili, J. (1972). Effects of isolated and enriched rearing on response inhibtion. Psychonomic Science, 27, 293-94.

Pacteau, C., Einon, D. \& Sinden, J. (1989). Early rearing environment and dorsal hippocampal ibotenic acid lesions: long term influences on spatial learning and alternation in the rat. Behavioural Brain Research, 34, 79-96.

Parsons, P. J. \& Spear, N. E. (1972). Long-term retention of avoidance learning by immature and adult rats as a function of environmental enrichment. Journal of Comparative and Physiological Psychology, 80 (2), 297-303.

Pope, S. (1991). Animal Expermentation Resource Material for Students. Animal Liberation Front: Victoria.

Rachlin, H. \& Green, L. (1972). Commitment, choice and self-control. Journal of the Experimental Analysis of Behaviour, 17: 15-22.

Raslear, T. G., Bauman, R. A., Hursh, S. R., Shurtleff, D. \& Simmons, L. (1988). Rapid demand curves for behavioural economics. Animal Learning and Behaviour, 16(3), 330-339. 
Renner, M. J. \& Rosenzweig, M. R. (1986). Object interactions in juvenile rats (Rattus norvegicus): effects of differential experiential histories. Journal of Comparative Psychology, 100(3), 229-236.*

Renner, M. J. \& Rosenzweig, M. R. (1987). Enriched and Impoverished Environments: Effects on Brain and Behavior. Springer-Verlag: New York.

Renner, M. J. \& Seltzer, C. P. (1991). Molar characteristics of exploratory and investigatory behavior int he rat (rattus norvegicus). Journal of Comparative Psychology, 105(4), 326-339.

Renner, M. J. \& Seltzer, C. P. (1994). Sequential structure in behavioral components of objects investigation by Long-Evans rat (rattus norvegicus). Journal of Comparative Psychology, 108(4), 335-343.

Robanovich, M. S. \& Rosvold, H. E. (1951). A close-field intelligence test for rats. Canadian Journal of Psychology, 5, 122-128.

Roeder, J. J., Chetcuti, Y. \& Will, B. (1980). Behavior and length of survival in populations of enriched and impoverished rats in the presence of a predator. Biology of Behavior, 5, 361-369.

Roper, T. J. (1973). Nesting material as a reinforcer for female mice. Animal Behaviour, 21, 733-740.

Rose, F. D. (1988). Environmental enrichment and recovery of function following brain damage in the rat. Medical Science Research, 16, 257-263.

Rose, M. A. (1990). Animal accomodation: is bigger better? ACCART News, 3(1), 8.

Rose, M. A. (1994). Environmental Factors Likely to Impact on an Animal's WellBeing-an Overview. Improving the Well-Being of Animals in the Research Environment 
Proceedings of the Conference Held at the Marriott Hotel, Sydney, October, 1993. ANZCCART, 99-116.

Rose, F. D., Davey, M. J., Al-Khamees, K. \& Attree E. A. (1992). General adaptive capacity and recovery of function following cortical damage in the rat. Medical Science Research, 20, 359-360.*

Roselinni, R. A. \& Widman, D. R. (1989). Prior exposure to stress reduces the diversity of exploratory behavior of novel objects in the rat (rattus norvegicus). Journal of Comparative Psychology, 103 (4), 339-346.

Sackett, G. P. (1967). Response to stimulus novelty and complexity as a function of rats' early rearing experiences. Journal of Comparative and Physiological Psychology, 63 (3), 369-375.

Schneider, S. (1988). Rats' behaviour in two different home cages. Humane Innovations and Alternatives in Animal Experimentation, 2, 39-42.

Schwartz, S. (1964). Effect of neonatal cortical lesions and early environmental factors on adult rat behavior. Journal of Comparative and Physiological Psychology, 57(1), 72-77.*

Shibagaki, M., Seo, M., Asano, T. \& Kiyono, S. (1981). Environmental enrichment to alleviate maze performance deficits in rats with microcephaly induced by $\mathrm{X}$-irradiation. Physiology and Behavior, 27, 797-802.*

Sherwin, C. M. (1996). Laboratory mice persist in gaining access to resources: a method of assessing importance of environmental features. Applied Animal Behavior Science, $48,103-214$.

Sherwin, C. M. \& Nicol, C. J. (1996). Reorganisation of behaviour in laboratory mice (mus musculus) with varying cost of access to resources. Animal Behaviour, 51, 1087-1093. 
Sherwin, C. M. \& Nicol, C. J. (1997). Behavioural demand functions of caged laboratory mice for additional space. Animal Behaviour, 53, 69-74.

Spence, J. T. \& Maher, B. A. (1962). Handling and noxious stimulation of the albino rats II. Effects on subsequent performance in a learning situation. Journal of Comparative and Physiological Psychology, 55(2), 252-255.

Smith, H. V. (1972). Effects of environmental enrichment on open-field activity and Hebb-Williams problem solving in rats. Journal of Comparative and Physiological Psychology, 80(1), 163-168.*

Sriklin, W. P. (1995). Space as an environmental enrichment. Laboratory Animals, 24(4), 24-27.

Timberlake, W. (1984). A temporal limit on the effect of future food on current performance in an analogue of foraging and welfare. Journal of the Experimental Analysis of Behavior, 41, 117-124.

Timberlake, W. \& Peden, B. F. (1987). On the distinction between open and closed economies. Journal of the Experimental Analysis of Behavior, 48, 35-60.

Townsend, P. (1997). Use of in-cage shelters by laboratory rats. Animal Welfare, 6, 95-103.

van Haaren, F. \& van Hest, A. (1989). Spatial matching and non-matching in male and female Wistar rats: Effects of delay interval duration. Animal Learning and Behavior, $17(3), 355-360$.

Venable, N., Pinto-Hamuy, T., Arratoa, J. A., Contador, M. T. Chellew, A. Pearn, C. \& Valenzuela, X. (1988). Greater efficacy of preweaning environmental enrichment on maze learning in rats. Behavioural Brain Research, 31, 89-92.* 
Walk, R. D. (1958) Visual and visual-motor experience: a replication. Journal of Comparative and Physiological Psychology, 51. 785-787.

Walk, R. D., Gibson, E. J., Pick, H. L. \& Tighe, T. J. (1958). Further experiments on prolonged exposure to visual forms: the effects of single stimuli and prior reinforcement. Journal of Comparative and Physiological Psychology, 51, 483-487.

Walsh, R. N. \& Cummins, R. A. (1976). The open field test: a critical review. Psychological Bulletin, 83, 482-504.

White, K. G. \& Alsop, B. (1993). In A. Sahagal (Ed.), Behavioural Neuroscience: a Practical Approach. I.R.L. Press: Oxford, 137-147.

Widman, D. R. \& Rosellini, R. A. (1990). Restricted daily exposure to environmental enrichment increases the diversity of exploration. Physiology and Behaviour, 47, 57-62.*

Will, B. E., Rosenzweig, M. R. \& Bennett, E. L. (1976). Effects of differential environments on recovery from neonatal brain lesions, measured by problem-solving scores and brain dimensions. Physiology and Behaviour, 16, 603-611.*

Will, B. E., Rosenzweig, M. R., Bennett, E. L., Hebert, M. \& Morimoto, H. (1977). Relatively brief environmental enrichment aids recovery of learning capacity and alters brain measures after postweaning brain lesions in rats. Journal of Physiological and Comparative Psychology, 91(1), 33-50**

Wood-gush, R. G. M. \& Vestergaard, K. (1991). The seeking of novelty and its relation to play. Animal Behaviour, 42, 599-606.

Woods, P. J. (1959). The effects of free and restricted environmental experience on problem-solving behavior in the rat. Journal of Physiological and Comparative Psychology, $52,399-402 * *$ 
Woods, P. J., Fiske, A. S. \& Ruckelshaus, S. I. (1961). The effects of drives conflicting with exploration on the problem-solving behavior of rats reared in free and restricted environments. Journal of Physiological and Comparative Psychology, 54(2), 167169.*

Woods, P. J., Ruckelshaus, S. I. \& Bowling, D. E. (1960). Some effects of "free" and "restricted" environmental rearing conditions on adult behavior in the rat. Psychological Reports, 6, 191-200**

Zajonc, R. (1972). Brainwash: familiarity breeds comfort. In Psychology Today (2nd ed.). C.R.M. Books: California.

Zimbardo, P. G. \& Montgomery, K. C. (1957). Effects of "free-environmental" rearing upon exploratory behavior. Psychological Reports, 3, 589-594.*

*Used to construct Table 1 


\section{APPENDIX 1}

Individual Data From Experiment 1; Emergence, Open Field and Hebb William's Maze (Relating to Figures 1-2 to 1-6)

\section{Emergence Latencies}

\begin{tabular}{|c|c|c|c|c|c|c|c|}
\hline el & $\begin{array}{l}5.72 \\
50.91 \\
162.34\end{array}$ & el & $\begin{array}{l}61.03 \\
65.15 \\
40.23\end{array}$ & sl & $\begin{array}{l}20.57 \\
15.75 \\
48.23\end{array}$ & dl & $\begin{array}{l}257.49 \\
155.89 \\
900\end{array}$ \\
\hline $\mathrm{se} 2$ & $\begin{array}{l}18.59 \\
20.54 \\
20.45\end{array}$ & e2 & $\begin{array}{l}77.57 \\
40.33 \\
32\end{array}$ & s2 & $\begin{array}{l}2.99 \\
29.67 \\
6.73\end{array}$ & d2 & $\begin{array}{l}194.93 \\
90.27 \\
46.77\end{array}$ \\
\hline se3 & $\begin{array}{l}9.97 \\
5.88 \\
5.77\end{array}$ & e3 & $\begin{array}{l}19.19 \\
7.2 \\
5.43\end{array}$ & s3 & $\begin{array}{l}10.99 \\
24.62 \\
19.57\end{array}$ & d3 & $\begin{array}{l}62.29 \\
8.63 \\
6.51\end{array}$ \\
\hline se 4 & $\begin{array}{l}5.54 \\
17.69 \\
12.02\end{array}$ & e4 & $\begin{array}{l}13.43 \\
63.9 \\
8.97\end{array}$ & s4 & $\begin{array}{l}21.07 \\
17.06 \\
5.68\end{array}$ & $\mathrm{~d} 4$ & $\begin{array}{l}900 \\
194.37 \\
88.09\end{array}$ \\
\hline ses & $\begin{array}{l}11.23 \\
4.56 \\
10.76\end{array}$ & e5 & $\begin{array}{l}29.85 \\
44.96 \\
33.01\end{array}$ & s5 & $\begin{array}{l}43.71 \\
123.87 \\
35.47\end{array}$ & $\mathrm{~d} 5$ & $\begin{array}{l}317.03 \\
34.22 \\
10.07\end{array}$ \\
\hline set & $\begin{array}{l}22.06 \\
118.54 \\
47.38\end{array}$ & e6 & $\begin{array}{l}14.85 \\
17.57 \\
7.8\end{array}$ & s6 & $\begin{array}{l}96.87 \\
24.51 \\
12.8\end{array}$ & d6 & $\begin{array}{l}473.53 \\
900 \\
900\end{array}$ \\
\hline $\mathrm{se} 7$ & $\begin{array}{l}10.69 \\
10.42 \\
7.72\end{array}$ & e7 & $\begin{array}{l}51.91 \\
51.36 \\
66.6\end{array}$ & s7 & $\begin{array}{l}15.32 \\
12.65 \\
10.85\end{array}$ & d7 & $\begin{array}{l}122.49 \\
5.88 \\
7.54\end{array}$ \\
\hline se8 & $\begin{array}{l}16.05 \\
16.6 \\
9.42\end{array}$ & e8 & $\begin{array}{l}10.53 \\
31.84 \\
17.47\end{array}$ & s8 & $\begin{array}{l}900 \\
114.34 \\
75.26\end{array}$ & & \\
\hline & & & & s9 & $\begin{array}{l}42.32 \\
14.99 \\
18.68\end{array}$ & & \\
\hline & & & & s10 & $\begin{array}{l}20.65 \\
14.06 \\
23.96\end{array}$ & & \\
\hline & & & & s11 & $\begin{array}{l}24.32 \\
.9 \\
4.47\end{array}$ & & \\
\hline & & & & $\mathrm{s} 12$ & $\begin{array}{l}3.84 \\
8.85 \\
10.55\end{array}$ & & \\
\hline
\end{tabular}

First Two Minute Activity Totals For Session One of the Open Field

$\begin{array}{lllllllll}\text { Enriched } & 50 & 75 & 119 & 101 & 95 & 51 & 96 & 85 \\ \text { Semi-E } & 64 & 105 & 94 & 62 & 61 & 80 & 91 & 58 \\ \text { Stand. 1 } & 52 & 67 & 50 & 58 & 68 & 80 & 73 & 49 \\ \text { Stand. 2 } & 56 & 48 & 82 & 56 & & & & \end{array}$


Deprived 51 32

58

35

50

66

55

Ten Minute Activity Totals For Session One of the Open Field

Enriched 146

237

* 281

\section{8}

$61 \quad 283$

$367 \quad 343$

329

337

241

164

199

371

200

Stand. $1 \quad 300$

324

258

342

185

229

166

32

286
243

192

223

205

Ten Minute Activity Totals For Session Two of the Open Field

$\begin{array}{ll}\text { Enriched } & 13 \\ \text { Semi-E } & 328 \\ \text { Stand. 1 } & 357 \\ \text { Stand. 2 } & 305 \\ \text { Deprived } & 355\end{array}$

$\begin{array}{ll}304 & * \\ 284 & 202 \\ 281 & 501 \\ 263 & 348 \\ 143 & 416\end{array}$

174
164
214
242
260

112

112

152

120

$202 \quad 164 \quad 178$

172

444

278

198

140

248

$\begin{array}{ll}\text { Stand. } 2 & 305 \\ \text { Deprived } & 355\end{array}$

258

335

131

Last Two Minute Activity Totals For Session Two of the Open Field

Enriched 13

Semi-E 60

Stand. $1 \quad 67$

Stand. $2 \quad 71$

Deprived 77

Enriched 172

Semi-E 305

Stand. $1 \quad 266$

Deprived 310

Enriched 19

Semi-E 67

Stand. $1 \quad 34$

Deprived 55

66
26
60
59
43

$*$
15
69
48
92

6
16
78
7
68

19

34

72

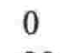

20

8

80

42

$52 \quad 32$

8

Ten Minute Totals For Session Three of the Open Field

$\begin{array}{llllll}210 & * & 160 & 229 & 99 & 138 \\ 329 & 215 & 222 & 172 & 66 & 416 \\ 345 & 427 & 410 & 405 & 125 & 182 \\ 315 & 388 & 286 & 317 & 343 & 272\end{array}$

Last Two Minute Totals For Session Three of the Open Field

$\begin{array}{lllllll}4 & * & 17 & 18 & 0 & 51 & 0 \\ 51 & 49 & 78 & 15 & 0 & 32 & 36 \\ 66 & 85 & 46 & 81 & 16 & 27 & 47 \\ 51 & 74 & 53 & 74 & 65 & 36\end{array}$

\section{Open Field Raw Data}

DAY ONE

Super-Enriched Rats

$\begin{array}{lllllllllllll}29 & 21 & 23 & 8 & 0 & 9 & 16 & 29 & 11 & 6 & 50 & 81 & 146 \\ 44 & 31 & 33 & 40 & 26 & 3 & 13 & 16 & 19 & 12 & 75 & 174 & 237 \\ 57 & 62 & 56 & * & * & * & * & * & * & * & 119 & * & * \\ 51 & 50 & 51 & 21 & 33 & 10 & 19 & 23 & 19 & 4 & 101 & 206 & 281 \\ 44 & 51 & 40 & 29 & 22 & 23 & 16 & 22 & 9 & 2 & 95 & 186 & 258 \\ 29 & 22 & 10 & 0 & 0 & 0 & 0 & 0 & 0 & 0 & 51 & 61 & 61 \\ 40 & 56 & 42 & 45 & 32 & 16 & 24 & 21 & 5 & 2 & 96 & 215 & 283 \\ 38 & 47 & 35 & 33 & 19 & 7 & 2 & 5 & 16 & 5 & 85 & 172 & 200\end{array}$

Enriched Rats

$\begin{array}{lllllllllllll}35 & 29 & 45 & 37 & 34 & 36 & 43 & 45 & 49 & 09 & 64 & 180 & 362 \\ 56 & 49 & 32 & 41 & 38 & 42 & 32 & 32 & 17 & 28 & 105 & 216 & 367\end{array}$


$\begin{array}{lllllllllllll}38 & 56 & 39 & 34 & 28 & 49 & 15 & 23 & 41 & 30 & 94 & 195 & 343\end{array}$

$\begin{array}{lllllllllllll}30 & 32 & 28 & 31 & 32 & 21 & 23 & 26 & 4 & 14 & 62 & 153 & 241\end{array}$

$\begin{array}{lllllllllllll}31 & 30 & 32 & 29 & 3 & 6 & 20 & 12 & 1 & 0 & 61 & 125 & 164\end{array}$

$\begin{array}{lllllllllllll}43 & 37 & 26 & 17 & 13 & 4 & 11 & 20 & 28 & 0 & 80 & 136 & 199\end{array}$

$\begin{array}{lllllllllllll}46 & 45 & 37 & 39 & 41 & 31 & 20 & 27 & 56 & 29 & 91 & 208 & 371\end{array}$

$\begin{array}{lllllllllllll}31 & 27 & 22 & 20 & 0 & 3 & 0 & 4 & 33 & 16 & 58 & 100 & 166\end{array}$

Standard Rats

$\begin{array}{lllllllllllll}29 & 23 & 29 & 32 & 40 & 44 & 21 & 21 & 29 & 32 & 52 & 153 & 300\end{array}$

$\begin{array}{lllllllllllll}29 & 38 & 35 & 43 & 26 & 32 & 30 & 29 & 34 & 33 & 67 & 171 & 329\end{array}$

$\begin{array}{lllllllllllll}20 & 30 & 23 & 45 & 25 & 37 & 39 & 49 & 35 & 34 & 50 & 143 & 337\end{array}$

$\begin{array}{lllllllllllll}20 & 38 & 21 & 32 & 21 & 20 & 25 & 22 & 41 & 18 & 58 & 132 & 258\end{array}$

$\begin{array}{lllllllllllll}34 & 34 & 48 & 42 & 40 & 48 & 28 & 38 & 28 & 32 & 68 & 198 & 342\end{array}$

$\begin{array}{lllllllllllll}46 & 34 & 33 & 13 & 1 & 26 & 17 & 14 & 1 & 0 & 80 & 127 & 185\end{array}$

$\begin{array}{lllllllllllll}37 & 36 & 31 & 23 & 32 & 22 & 22 & 16 & 5 & 37 & 73 & 159 & 229\end{array}$

$\begin{array}{lllllllllllll}25 & 24 & 40 & 40 & 26 & 37 & 18 & 28 & 38 & 41 & 49 & 155 & 317\end{array}$

Standard Rats (second control)

$\begin{array}{lllllllllllll}22 & 34 & 39 & 30 & 38 & 32 & 22 & 12 & 24 & 40 & 56 & 163 & 293\end{array}$

$\begin{array}{lllllllllllll}22 & 26 & 39 & 43 & 27 & 27 & 42 & 33 & 35 & 30 & 48 & 157 & 324\end{array}$

$\begin{array}{lllllllllllll}42 & 40 & 43 & 29 & 16 & 39 & 31 & 28 & 9 & 9 & 82 & 170 & 286\end{array}$

$\begin{array}{lllllllllllll}32 & 24 & 24 & 14 & 18 & 30 & 26 & 2 & 2 & 2 & 56 & 112 & 168\end{array}$

Deprived Rats

$\begin{array}{lllllllllllll}23 & 28 & 29 & 27 & 24 & 33 & 30 & 30 & 26 & 21 & 51 & 131 & 271\end{array}$

$\begin{array}{lllllllllllll}28 & 4 & 0 & 0 & 0 & 0 & 0 & 0 & 0 & 0 & 32 & 32 & 32\end{array}$

$\begin{array}{lllllllllllll}27 & 31 & 33 & 20 & 14 & 13 & 25 & 30 & 23 & 27 & 58 & 125 & 243\end{array}$

$\begin{array}{lllllllllllll}15 & 20 & 27 & 31 & 18 & 30 & 27 & 10 & 4 & 20 & 35 & 111 & 202\end{array}$

$\begin{array}{lllllllllllll}22 & 28 & 22 & 21 & 23 & 16 & 22 & 14 & 13 & 11 & 50 & 116 & 192\end{array}$

$\begin{array}{lllllllllllll}34 & 32 & 22 & 24 & 20 & 24 & 15 & 19 & 19 & 14 & 66 & 132 & 223\end{array}$

$\begin{array}{lllllllllllll}22 & 33 & 27 & 23 & 12 & 15 & 14 & 14 & 25 & 20 & 55 & 117 & 205\end{array}$

Super-enriched Rats

DAY TWO

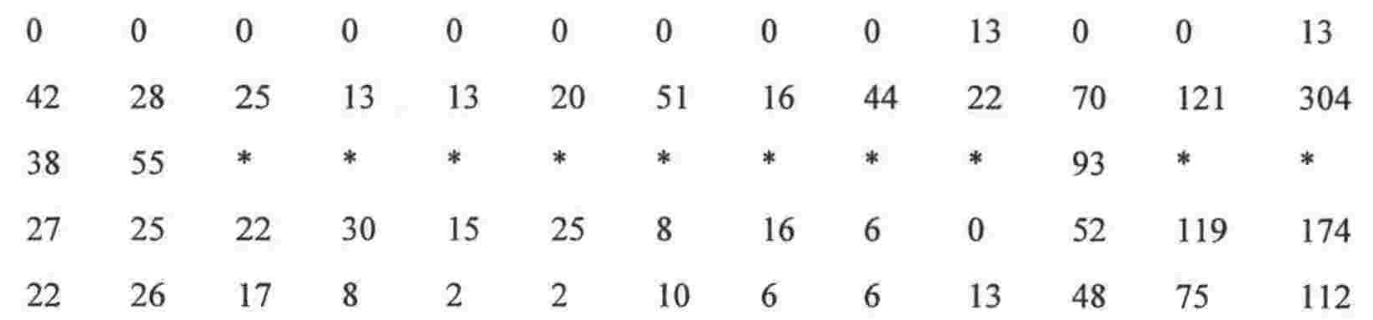




$\begin{array}{lllllllllllll}12 & 31 & 16 & 23 & 17 & 13 & 0 & 0 & 0 & 0 & 43 & 99 & 112 \\ 34 & 27 & 33 & 28 & 12 & 4 & 0 & 6 & 4 & 4 & 61 & 134 & 152 \\ 20 & 23 & 19 & 26 & 6 & 0 & 0 & 5 & 11 & 10 & 43 & 94 & 120\end{array}$

Enriched rats

$\begin{array}{lllllllllllll}25 & 31 & 36 & 33 & 34 & 32 & 36 & 41 & 25 & 35 & 56 & 159 & 328 \\ 38 & 30 & 29 & 46 & 14 & 38 & 34 & 24 & 18 & 8 & 68 & 157 & 284 \\ 35 & 35 & 36 & 35 & 29 & 20 & 48 & 9 & 7 & 8 & 70 & 170 & 202 \\ 27 & 9 & 8 & 17 & 5 & 37 & 33 & 12 & 6 & 10 & 36 & 66 & 164 \\ 32 & 31 & 21 & 8 & 18 & 10 & 0 & 24 & 16 & 18 & 63 & 110 & 178 \\ 18 & 19 & 13 & 19 & 0 & 0 & 0 & 20 & 16 & 4 & 37 & 69 & 172 \\ 66 & 38 & 58 & 48 & 50 & 41 & 33 & 30 & 34 & 46 & 104 & 260 & 444 \\ 28 & 16 & 30 & 25 & 41 & 25 & 7 & 0 & 0 & 0 & 44 & 106 & 109\end{array}$

Standard rats

$\begin{array}{lllllllllllll}31 & 45 & 34 & 44 & 26 & 25 & 45 & 40 & 35 & 32 & 76 & 180 & 357 \\ 24 & 52 & 19 & 35 & 28 & 35 & 15 & 13 & 38 & 22 & 76 & 158 & 281 \\ 32 & 41 & 60 & 62 & 58 & 53 & 62 & 44 & 30 & 39 & 73 & 253 & 501 \\ 27 & 21 & 8 & 12 & 10 & 4 & 18 & 36 & 48 & 30 & 48 & 78 & 214 \\ 12 & 15 & 6 & 28 & 44 & 25 & 32 & 44 & 35 & 37 & 27 & 105 & 278 \\ 48 & 40 & 16 & 6 & 17 & 16 & 21 & 8 & 16 & 10 & 88 & 127 & 198 \\ 19 & 16 & 15 & 14 & 25 & 9 & 4 & 0 & 4 & 38 & 35 & 89 & 140 \\ 12 & 18 & 31 & 43 & 23 & 18 & 35 & 37 & 26 & 5 & 30 & 127 & 248\end{array}$

Standard Rats (second control)

$\begin{array}{lllllllllllll}26 & 38 & 26 & 44 & 30 & 19 & 19 & 32 & 31 & 40 & 64 & 164 & 305 \\ 36 & 32 & 32 & 18 & 10 & 25 & 30 & 21 & 23 & 36 & 68 & 128 & 263 \\ 36 & 46 & 37 & 42 & 38 & 43 & 36 & 22 & 20 & 28 & 82 & 199 & 348 \\ 41 & 26 & 46 & 29 & 26 & 31 & \underline{16} & 20 & 2 & 5 & 67 & 168 & 242\end{array}$

Deprived Rats

$\begin{array}{lllllllllllll}23 & 45 & 33 & 37 & 34 & 16 & 37 & 53 & 29 & 48 & 68 & 172 & 355 \\ 6 & 10 & 19 & 17 & 9 & 4 & 12 & 23 & 27 & 16 & 16 & 61 & 143 \\ 50 & 47 & 47 & 32 & 38 & 48 & 27 & 35 & 42 & 50 & 97 & 214 & 416 \\ 22 & 28 & 28 & 32 & 18 & 10 & 30 & 24 & 39 & 29 & 50 & 128 & 260 \\ 22 & 28 & 22 & 18 & 30 & 41 & 28 & 17 & 31 & 21 & 50 & 120 & 258 \\ 56 & 20 & 37 & 31 & 35 & 48 & 31 & 45 & 29 & 3 & 76 & 179 & 335 \\ 9 & 33 & 18 & 16 & 12 & 12 & 17 & 6 & 4 & 4 & 42 & 88 & 131\end{array}$


Super-enriched Rats

$\begin{array}{lllllllllllll}13 & 17 & 18 & 31 & 8 & 20 & 32 & 14 & 15 & 4 & 30 & 87 & 172\end{array}$

$\begin{array}{lllllllllllll}41 & 29 & 47 & 16 & 15 & 35 & 11 & 12 & 4 & 0 & 70 & 148 & 210\end{array}$

$\begin{array}{lll}45 & 35 & 29\end{array}$

$\begin{array}{lllllllllllll}22 & 13 & 22 & 16 & 11 & 32 & 24 & 3 & 12 & 5 & 35 & 84 & 160\end{array}$

$\begin{array}{lllllllllllll}34 & 18 & 15 & 10 & 36 & 42 & 29 & 27 & 7 & 11 & 51 & 113 & 229\end{array}$

$\begin{array}{lllllllllllll}10 & 14 & 10 & 27 & 24 & 10 & 4 & 0 & 0 & 0 & 24 & 85 & 99\end{array}$

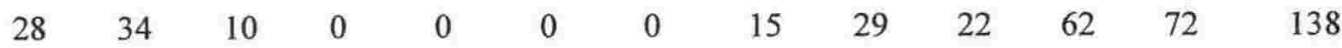

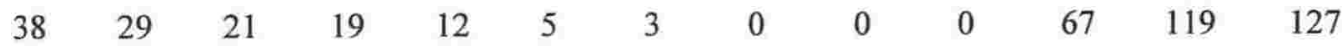

Enriched rats

$\begin{array}{lllllllllllll}30 & 36 & 45 & 36 & 34 & 38 & 15 & 4 & 34 & 33 & 66 & 181 & 305\end{array}$

$\begin{array}{lllllllllllll}54 & 21 & 29 & 41 & 34 & 40 & 34 & 25 & 35 & 16 & 75 & 179 & 329\end{array}$

$\begin{array}{lllllllllllll}25 & 20 & 26 & 34 & 41 & 25 & 25 & 0 & 19 & 30 & 45 & 146 & 215\end{array}$

$\begin{array}{lllllllllllll}30 & 30 & 10 & 18 & 14 & 28 & 0 & 28 & 36 & 42 & 60 & 102 & 222\end{array}$

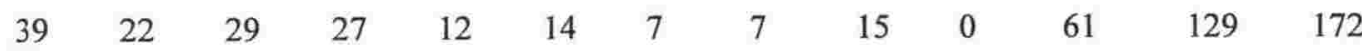

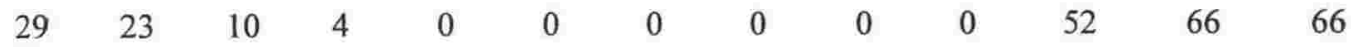

$\begin{array}{lllllllllllll}74 & 57 & 46 & 44 & 33 & 31 & 28 & 41 & 37 & 25 & 131 & 254 & 416\end{array}$

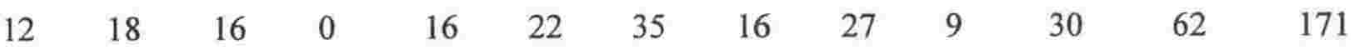

Standard rats

$\begin{array}{lllllllllllll}46 & 27 & 46 & 25 & 8 & 23 & 24 & 33 & 0 & 34 & 73 & 152 & 266 \\ 54 & 42 & 36 & 37 & 40 & 40 & 33 & 37 & 29 & 37 & 96 & 206 & 345 \\ 50 & 47 & 37 & 37 & 44 & 54 & 29 & 44 & 50 & 35 & 97 & 215 & 427 \\ 38 & 55 & 62 & 64 & 45 & 34 & 42 & 24 & 20 & 26 & 93 & 264 & 410 \\ 26 & 28 & 35 & 54 & 53 & 50 & 44 & 34 & 48 & 33 & 54 & 196 & 405 \\ 40 & 31 & 18 & 14 & 6 & 0 & 0 & 0 & 0 & 16 & 71 & 109 & 125 \\ 22 & 16 & 10 & 22 & 7 & 44 & 23 & 11 & 27 & 0 & 38 & 77 & 182 \\ 12 & 29 & 45 & 46 & 34 & 39 & 51 & 26 & 28 & 19 & 41 & 166 & 329\end{array}$

Deprived rats

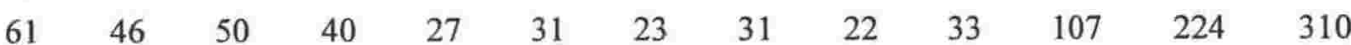

$\begin{array}{lllllllllllll}6 & 30 & 32 & 31 & 41 & 40 & 39 & 45 & 27 & 24 & 36 & 140 & 315\end{array}$

$\begin{array}{lllllllllllll}48 & 34 & 35 & 37 & 37 & 52 & 47 & 24 & 30 & 44 & 82 & 191 & 388\end{array}$

$\begin{array}{lllllllllllll}30 & 25 & 31 & 32 & 43 & 13 & 28 & 31 & 39 & 14 & 55 & 161 & 286\end{array}$

$\begin{array}{lllllllllllll}30 & 30 & 37 & 26 & 45 & 31 & 35 & 19 & 34 & 30 & 60 & 168 & 317\end{array}$

$\begin{array}{lllllllllllll}44 & 42 & 49 & 41 & 22 & 14 & 31 & 35 & 25 & 40 & 82 & 194 & 343\end{array}$ 
$\begin{array}{lllllllllllll}34 & 26 & 33 & 30 & 33 & 31 & 24 & 25 & 20 & 16 & 60 & 156 & 272\end{array}$

Initial Errors Made in the Hebb William's Maze

\begin{tabular}{|c|c|c|c|c|c|c|c|c|}
\hline Enriched & 49 & 48 & 53 & 46 & 41 & 41 & 41 & 41 \\
\hline Semi-E & 41 & 42 & 43 & 45 & 50 & 48 & 48 & 43 \\
\hline Stand. 1 & 35 & 56 & 46 & 39 & 49 & 43 & 45 & 44 \\
\hline Stand. 2 & 47 & 50 & 48 & 48 & & & & \\
\hline Deprived & 42 & 42 & 54 & 46 & 57 & 52 & 50 & \\
\hline \multicolumn{9}{|c|}{ Repeated Errors Made in the Hebb William's Maze } \\
\hline Enriched & 125 & 142 & 129 & 118 & 102 & 89 & 123 & 115 \\
\hline Semi-E & 187 & 116 & 141 & 188 & 145 & 144 & 149 & 55 \\
\hline Stand. 1 & 147 & 249 & 155 & 163 & 181 & 118 & 188 & 143 \\
\hline Stand. 2 & 178 & 170 & 184 & 175 & & & & \\
\hline Deprived & 131 & 220 & 229 & 149 & 158 & 128 & 155 & \\
\hline
\end{tabular}




\section{APPENDIX 2.1}

Individual Raw Data From Experiment 2.1: Correct Choices: T-Maze and Experimental Chamber (Relating to Figures 2.1, and 2.2).

\section{Memory (T-maze)}

\begin{tabular}{lllllllllll} 
Enriched & \multicolumn{7}{c}{ Deprvd } \\
5334 & 5424 & 4443 & 4334 & 5244 & 3232 & 4221 & 3544 & 5344 & 5343 & 4323 \\
3203 & 4211 & 4333 & 5332 & 4522 & 5423 & 5342 & 2513 & 5431 & 5334 & 5223 \\
4133 & 5455 & 5455 & 5333 & 2223 & 3351 & 4523 & 4244 & 5342 & 3343 & 5312 \\
5324 & 3444 & 5333 & 5454 & 4333 & 5343 & 3334 & 5333 & 5232 & 4041 & 5433 \\
5331 & 5334 & 5553 & 5325 & 4143 & 3122 & 5233 & 5312 & 4322 & 2313 & 5231 \\
4452 & 5444 & 5553 & 5452 & 5422 & 4224 & 2433 & 5343 & 4424 & 5224 & 2334 \\
3134 & 5534 & 5414 & 4435 & 5232 & 5334 & 3541 & 4231 & 3422 & 5230 & 4333 \\
4445 & 5333 & 5424 & 4323 & 4433 & 3331 & 4133 & 5232 & 4432 & 4111 & 2244 \\
4121 & 5544 & 4335 & 4212 & 3224 & 5244 & 4453 & 3124 & 5212 & 2232 & 5321 \\
4312 & 5433 & 5454 & 4312 & 2422 & 4134 & 5221 & 4133 & 3543 & 3132 & 4233 \\
4313 & 5433 & 3541 & 2433 & 4124 & 5331 & 5223 & 5342 & 5233 & 3323 & 4332 \\
2322 & 5325 & 4433 & 5313 & 5113 & 5234 & 4335 & 5341 & 4412 & 5233 & 4224 \\
4421 & 5425 & 5353 & 3312 & 4224 & 5434 & 1423 & 3231 & 5233 & 4422 & 5321 \\
4214 & 5213 & 3422 & 5343 & 4323 & 5444 & 3233 & 2351 & 3332 & 5443 & 3233 \\
5422 & 5332 & 5132 & 4332 & 5233 & 5444 & 5421 & 4322 & 4222 & 5322 & 4322 \\
5343 & 5534 & 5143 & 5342 & 4132 & 3324 & 3342 & 3323 & 3324 & 4231 & 5231 \\
4535 & 3242 & 2420 & 4342 & 1142 & 5253 & 3422 & 4403 & 5315 & 4222 & 4322 \\
4321 & 4213 & 4442 & 5342 & 3323 & 5224 & 4444 & 5143 & 4232 & 4145 & 5232 \\
4222 & 5242 & 4431 & 3201 & 3434 & 4334 & 4322 & 4322 & 3241 & 4422 & 5332 \\
3345 & 5333 & 3233 & 4145 & 4333 & 4321 & 2243 & 5232 & 4442 & 3231 & 5334 \\
5243 & 3533 & 4345 & 5342 & 4333 & 4222 & 3322 & 4133 & 3321 & 3311 & 5233 \\
4223 & 4213 & 5333 & 4334 & 3421 & 4522 & 2322 & 4332 & 2323 & 5032 & 5443 \\
4324 & 3404 & 4531 & 3543 & 4242 & 4313 & 2323 & 5355 & 3343 & 5341 & 5423
\end{tabular}

\section{Memory (Operant)}

LeftCorrect LeftError RightCorrect RightError 4 Delays Deprived Rats

A 4724222 365203
B

591609 342234
C

5834510 290229
D

673590 240390
E

623511 174381
F

3412440 175211 


$\begin{array}{llllll}286203 & 246268 & 2631714 & 342262 & 279223 & 169211 \\ \begin{array}{l}19908 \\ \text { Enriched Rats }\end{array} & 24101911 & 217209 & 2551717 & 1215294 & 192194 \\ \text { A } & \text { B } & \text { C } & \text { D } & \text { E } & \text { F } \\ 574586 & 602439 & 313353 & 5610561 & 290312 & 560507 \\ 254303 & 230249 & 150174 & 241313 & 170131 & 263232 \\ 1719280 & 2351811 & 1551811 & 270235 & 153131 & 233282 \\ 824301 & 1991414 & 127116 & 257214 & 77153 & 195218 \\ 5 \text { Delays } & & & & & \\ \text { Deprived } & & & & & \\ 210143 & 261202 & 140123 & 203312 & 181151 & 251101 \\ 141203 & 261192 & 21173 & 293157 & 97190 & 142175 \\ 16794 & 223195 & 93154 & 2241217 & 129120 & 172148 \\ 106138 & 1281811 & 95125 & 2261113 & 85166 & 24359 \\ 89194 & 1671214 & 67810 & 196821 & 118115 & 871112 \\ \text { Enriched } & & & & & \\ 160215 & 192215 & 231184 & 290235 & 240170 & 13291 \\ 230171 & 200171 & 210184 & 280256 & 211181 & 130121 \\ 175163 & 174163 & 211168 & 322187 & 153211 & 101122 \\ 146203 & 188163 & 188161 & 3041217 & 138155 & 94123 \\ 1110162 & 814172 & 1111176 & 241924 & 88196 & 102311\end{array}$


APPENDIX 2.2

Individual Response Ratio Data From Experiment 2.2: Response in S1/Total Responding (Relating to Figures $2.3,2.4,2.5$ and 2.6).

\begin{tabular}{|c|c|c|c|c|c|}
\hline$\frac{\text { Subject1 }}{\text { Schedules }}$ & Subject2 & $\underline{\text { Subject3 }}$ & $\underline{\text { Subject4 }}$ & $\underline{\text { Subject5 }}$ & $\underline{\text { Subject6 }}$ \\
\hline \multicolumn{6}{|c|}{ Rich objects } \\
\hline 1.17771 & 1.05178 & 1.09892 & .87454 & .92614 & .55959 \\
\hline .72378 & .91201 & 1.10152 & .95728 & 1.03687 & 2.45491 \\
\hline .65066 & .92032 & 1.11892 & .94846 & 1.10033 & .87394 \\
\hline \multicolumn{6}{|c|}{ Lean Objects } \\
\hline 1.11457 & .73721 & .75264 & .91375 & .75794 & 1.14559 \\
\hline .88867 & .81432 & .76296 & .59166 & .92214 & .91837 \\
\hline .48932 & .76296 & .74823 & .64563 & .85821 & .84590 \\
\hline \multicolumn{6}{|c|}{ Rich Pre-F } \\
\hline .84036 & .74920 & 1.01176 & .87305 & .87747 & .87998 \\
\hline .83218 & .77634 & .70173 & .96875 & .82144 & .99074 \\
\hline .07473 & 1.01454 & .09193 & 1.00578 & .54057 & 1.24550 \\
\hline .01294 & .01082 & .04236 & .01682 & .14261 & .46089 \\
\hline .42827 & .11821 & .16113 & .02685 & .03867 & .41421 \\
\hline \multicolumn{6}{|c|}{ Lean Pre-F } \\
\hline .37043 & .79167 & .93952 & 1.00175 & .87439 & .82164 \\
\hline .39575 & .63904 & .37441 & 1.05379 & .68923 & .85304 \\
\hline .05687 & .60201 & .07415 & .94806 & .35219 & .93844 \\
\hline .02418 & .01204 & .02140 & .00457 & .07382 & .12783 \\
\hline .17515 & .20109 & .18140 & .02397 & .02610 & .16648 \\
\hline \multicolumn{6}{|c|}{ Rich Extinc } \\
\hline .38314 & .53895 & .36079 & .61072 & 1.22794 & .53267 \\
\hline .57365 & .29061 & .28348 & .29340 & .50210 & .51830 \\
\hline .20822 & .10541 & .08113 & .08664 & .38971 & .09484 \\
\hline .32407 & .38890 & .28500 & .39246 & .67041 & .29019 \\
\hline .59877 & .19316 & .24581 & .16647 & .42528 & .24683 \\
\hline .20679 & .09074 & .11667 & .20132 & .25399 & .04003 \\
\hline
\end{tabular}

Effect of the Disrupters in the Order; Objects, Prefeeding Extinction

$$
\text { Group } 1
$$

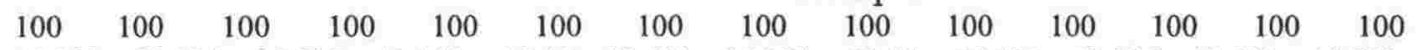

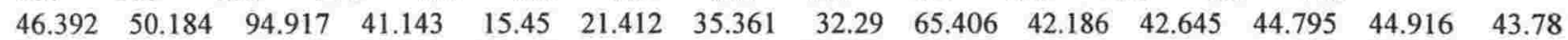

$\begin{array}{llllllllllllll}24.189 & 22.994 & 46.369 & 38.256 & 7.1063 & 28.762 & 59.1 & 26.391 & 44.297 & 39.673 & 27.039 & 25.538 & 27.946 & 37.006\end{array}$

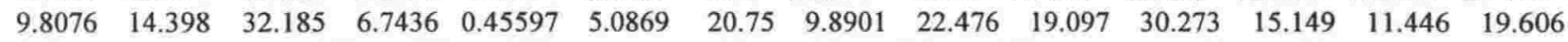

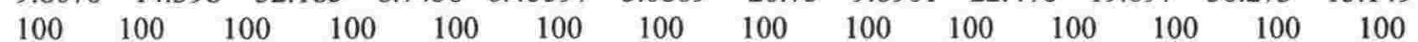

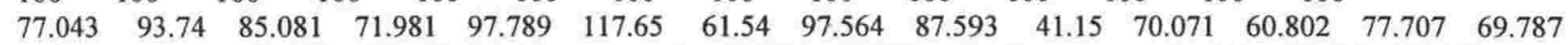

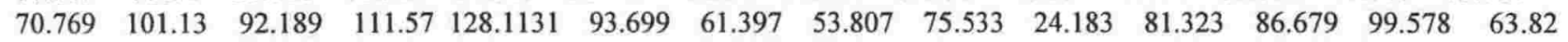
$\begin{array}{llllllllllllll}8.304 & 51.919 & 51.48 & 61.865 & 42.98 & 32.086 & 6.58 & 44.638 & 49.752 & 7.4919 & 45.852 & 28.493 & 41.439 & 30.468\end{array}$

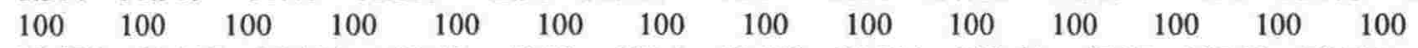

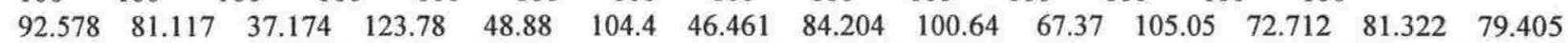

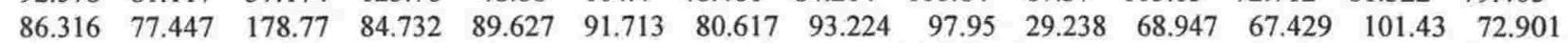

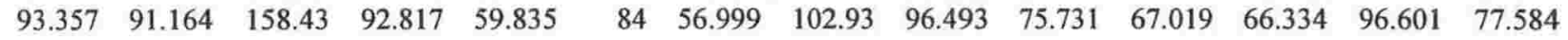

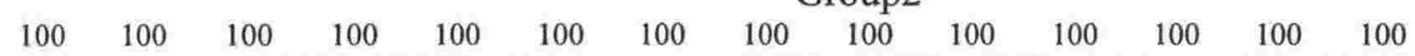

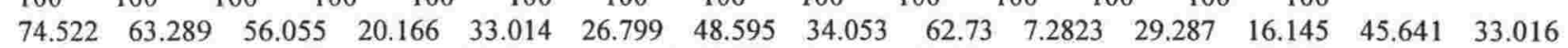

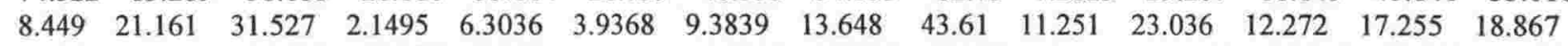

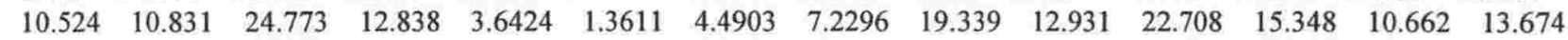




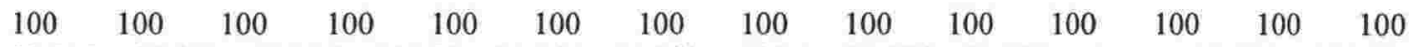

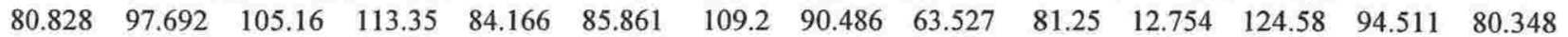

$\begin{array}{llllllllllllll}22.878 & 28.959 & 54.118 & 16.607 & 32.361 & 56.926 & 27.192 & 9.303 & 35.237 & 2.5953 & 4.9229 & 40.253 & 35.308 & 19.917\end{array}$

$\begin{array}{llllllllllllll}30.171 & 15.964 & 29.034 & 89.117 & 14.888 & 13.276 & 17.127 & 2.5407 & 6.7366 & 0.78983 & 2.8238 & 7.0017 & 32.075 & 6.1698\end{array}$

$\begin{array}{llllllllllllll}1.8563 & 1.159 & 29.436 & 0.081335 & 69.716 & 1.4147 & 3.1881 & 1.0696 & 11.832 & 1.2337 & 20.821 & 5.3479 & 17.277 & 7.2487\end{array}$

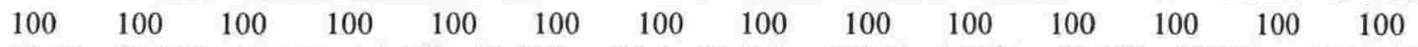

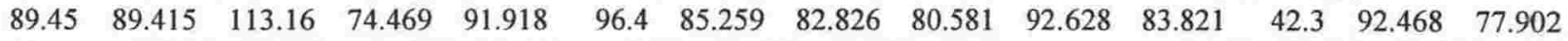

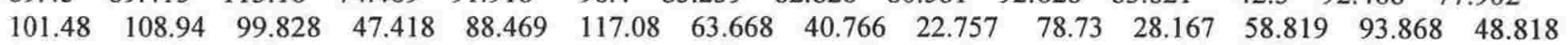

$\begin{array}{llllllllllllll}142.12 & 79.705 & 68.358 & 159.73 & 71.229 & 119.27 & 72.032 & 133.63 & 41.974 & 122.76 & 86.69 & 109.82 & 106.67 & 94.484\end{array}$ 
APPENDIX 2.3

Individual Matching Line Slopes and Biases and Ratios of Responding to S1/total From Experiment 2.3 (Relating to Figures 2.7 and 2.8).

Visual Discrimination

1

Enriched

0.489664

0.496139

0.462347

0.473161

0.438487

0.390593

0.370664

0.346711

0.320818

0.304728

Deprived

0.471736

0.401661

0.414078

0.359159

0.346183

0.351828

0.363657

0.348756

0.326278

0.301143

0.532579
0.485494
0.476241
0.447078
0.384805
0.375168
0.349018
0.326586
0.29639
0.332089

0.505216
0.294849
0.319048
0.306462
0.338933
0.298485
0.343596
0.37277
0.271163
0.238609

0.495946
0.47514
0.419245
0.412955
0.329171
0.288261
0.251567
0.210805
0.228239
0.254641

0.575926

0.547802

0.490928

0.494234

0.468242

0.423846

0.412573

0.363754

0.408408

0.367554

0.392956

0.400688

0.49839

0.351272

0.424213

0.425084

0.314589

0.451581

0.352408

0.427879

0.305151

0.265493

0.458263

0.291505

0.439892

0.414508

0.294302

0.269309

0.379827

0.265447

\subsection{6}

0.515582

0.448133

0.431798

0.488514

0.486568

0.388459

0.363289

0.443286

0.396609

0.433359

0.423496

0.411541

0.336991

0.327011

0.43988

0.419242

0.345977

0.338445

0.335448

0.344279

0.412703

0.378447

0.446323

0.373094

0.35943

0.322387

0.405019

0.384235

0.346125

0.366743

0.361525

0.428776

0.39253

0.334082

0.325804

0.349893

0.324687

0.301875

2

enriched

0.370411

0.477578

0.517333

0.512043

0.544458

deprived

0.552886

0.446542

0.59869

0.650039

0.66566

0.700648

0.748309

0.379884

0.335584

0.300097

0.393194

0.307916

0.408321

0.441548

0.491489

0.488718

0.525261

0.470673

0.629802

0.520158

0.54175

0.600615

0.531357

0.637783

0.615273

0.615115

0.664622

0.57092

0.66266

0.577879

0.598418

0.61971

0.451473

0.432881

0.418455

0.437733

0.584139

0.494519

0.503505

0.686221

0.483078

0.557643

0.551881

0.672831

0.505851

0.57158

0.62443

0.759601

0.618617

0.646521

0.669134

0.768963

0.658393

0.62208

0.643077

3

enriched

0.530988

0.486631

0.461006

0.418249

0.376781

0.461507

deprived

0.425933

0.314139

0.300747

0.469778
0.437136
0.412703
0.39492
0.372239
0.354672

0.418859
0.25468
0.245606

0.495385
0.331964
0.297121
0.346364
0.319741
0.26624
0.515459
0.423701
0.389898

0.669391
0.577587
0.513241
0.405748
0.371834
0.440607

0.565608
0.483177
0.46612

0.577561

0.538968

0.490648

0.39564

0.487961

0.37863

$0.433632 \quad 0.353613$

$0.469923 \quad 0.391484$

$0.472437 \quad 0.299263$

$0.552163 \quad 0.534597$

$0.412358 \quad 0.45083$

$0.442702 \quad 0.41674$ 


$\begin{array}{llllll}0.283357 & 0.224579 & 0.379875 & 0.445923 & 0.387921 & 0.391447 \\ 0.331016 & 0.31124 & 0.376998 & 0.437648 & 0.389266 & 0.36297 \\ 0.344536 & 0.247017 & 0.36916 & 0.385101 & 0.378612 & 0.368066\end{array}$

\section{Matching}

$\begin{array}{lllll}\text { biast } & \text { enriched } & \text { semi-e } & \text { standard } & \text { deprived } \\ 1 & .00563 & .04314 & -.01972 & -.07923 \\ 2 & -.09068 & -.11785 & -.15748 & -.02139 \\ 3 & -.00261 & .05448 & -.01361 & -.04995 \\ 4 & -.04838 & -.12888 & -.04729 & -.03684 \\ 5 & -.1178 & .08718 & & -.02946 \\ 6 & -.09033 & -.11364 & & -.11204 \\ 7 & -.05057 & .03232 & & -.06323 \\ 8 & -.00004 & -.09042 & & \\ \text { slope } & & & & \\ 1 & .033 & -.03054 & .07351 & .20987 \\ 2 & .11576 & .19462 & .50063 & .39829 \\ 3 & .02132 & .01399 & .19737 & .08376 \\ 4 & .14675 & .14299 & .22267 & .37404 \\ 5 & .16306 & .41313 & & .10836 \\ 6 & .10396 & .14478 & & .28025 \\ 7 & .18898 & .17572 & & .18454 \\ 8 & .03552 & .13189 & & \end{array}$


APPENDIX 2.4

Herrnstein's Hyperbola Individual $K$ and Ro Values from Experiment 2.4 (Relating to Figure 2.9)

Enriched Box 7 Deprived Box 8

$\begin{array}{llllll}1 & 2 & 1 & 2 & 3 & 4 \\ \text { FR240 } & & & & & \\ 146 & 43 & 89 & 299 & 181 & 413 \\ 62 & 85 & 101 & 250 & 113 & 256 \\ 26 & 62 & 93 & 367 & 44 & 281 \\ 89 & 60 & 103 & 307 & 107 & 274 \\ 61 & 39 & 108 & 252 & 218 & 189 \\ \mathbf{7 6 . 8} & \mathbf{5 7 . 8} & \mathbf{9 8 . 8} & \mathbf{2 9 5} & \mathbf{1 3 2 . 6} & \mathbf{2 8 2 . 6} \\ \text { FR150 } & & & & & \\ 140 & 84 & 166 & 349 & 284 & 217 \\ 89 & 112 & 157 & 270 & 297 & 116 \\ 113 & 112 & 97 & 266 & 356 & 123 \\ 100 & 41 & 133 & 227 & 293 & 123 \\ 42 & 90 & 198 & 235 & 407 & 197 \\ \mathbf{9 6 . 8} & \mathbf{8 7 . 8} & \mathbf{1 5 0 . 2} & \mathbf{2 6 9 . 4} & \mathbf{3 2 7 . 4} & \mathbf{1 9 9 . 8} \\ \text { FR75 } & & & & & \\ 254 & 137 & 140 & 398 & 385 & 320 \\ 161 & 95 & 205 & 235 & 347 & 259 \\ 364 & 140 & 180 & 403 & 314 & 283 \\ 321 & 171 & 69 & 404 & 247 & 379 \\ 223 & 175 & 155 & 327 & 263 & 379 \\ \mathbf{2 6 4 . 6} & \mathbf{1 4 3 . 6} & \mathbf{1 4 9 . 8} & \mathbf{2 7 9 . 2} & \mathbf{3 1 1 . 2} & \mathbf{3 2 4} \\ \text { FR30 } & & & & & \\ 562 & 497 & 336 & 544 & 477 & 692 \\ 449 & 602 & 242 & 554 & 310 & 650 \\ 695 & 403 & 238 & 570 & 535 & 608 \\ 560 & 531 & 318 & 648 & 582 & 494 \\ 503 & 430 & 307 & 633 & 535 & 500 \\ \mathbf{5 5 2 . 8} & \mathbf{4 9 2 . 6} & \mathbf{2 5 7} & \mathbf{5 9 0 . 2} & \mathbf{4 8 7 . 8} & \mathbf{5 8 8 . 8} \\ \text { FR10 } & & & & & \\ 494 & 352 & 152 & 388 & 97 & 650 \\ 694 & 392 & 222 & 363 & 351 & 523 \\ 725 & 409 & 315 & 512 & 362 & 580 \\ 654 & 436 & 364 & 572 & 295 & 614 \\ 751 & 404 & 291 & 422 & 241 & 618 \\ \mathbf{6 6 3 . 6} & \mathbf{3 9 8 . 6} & \mathbf{2 6 8 . 8} & \mathbf{4 5 1 . 4} & \mathbf{2 6 9 . 2} & \mathbf{5 9 7} \\ \text { FR5 } & & & & & \\ 635 & 397 & 241 & 426 & 278 & 595 \\ 541 & 337 & 302 & 452 & 292 & 582 \\ 609 & 308 & 363 & 440 & 360 & 486 \\ 652 & 411 & 352 & 466 & 282 & 619 \\ 643 & 352 & 319 & 529 & 294 & 690\end{array}$

Enriched Box $8 \quad$ Deprived Box 8

FR240

$\begin{array}{llllll}1 & 2 & 3 & 4 & 5 & 6 \\ 237 & 209 & 260 & 77 & 185 & 205 \\ 88 & 146 & 260 & 29 & 327 & 237 \\ 181 & 190 & 125 & 20 & 159 & 235 \\ 140 & 171 & 219 & 156 & 359 & 187 \\ 136 & 64 & 196 & 81 & 276 & 149 \\ \mathbf{1 5 6 . 4} & \mathbf{1 5 6} & \mathbf{2 1 2} & \mathbf{7 2 . 6} & \mathbf{2 6 1 . 2} & \mathbf{2 0 1 . 6} \\ \text { FR150 } & & & & & \\ 88 & 84 & 232 & 243 & 193 & 82\end{array}$




$\begin{array}{llllll}118 & 93 & 245 & 266 & 318 & 257 \\ 80 & 102 & 213 & 232 & 315 & 192 \\ 139 & 75 & 73 & 301 & 199 & 143 \\ 142 & 85 & 201 & 21 & 397 & 219 \\ \mathbf{1 1 3 . 4} & \mathbf{8 7 . 8} & \mathbf{1 9 2 . 8} & \mathbf{2 1 2 . 6} & \mathbf{2 8 4 . 4} & \mathbf{1 7 8 . 6} \\ \text { FR75 } & & & & & \\ 179 & 443 & 285 & 357 & 301 & 160 \\ 246 & 316 & 102 & 468 & 644 & 190 \\ 264 & 343 & 188 & 312 & 672 & 199 \\ 154 & 158 & 234 & 414 & 437 & 210 \\ 138 & 205 & 234 & 306 & 410 & 196 \\ \mathbf{1 9 6 . 2} & \mathbf{2 9 3} & \mathbf{2 0 8 . 6} & \mathbf{3 7 1 . 4} & \mathbf{4 9 2 . 8} & \mathbf{1 9 1} \\ \text { FR30 } & & & & & \\ 570 & 276 & 526 & 655 & 748 & 239 \\ 403 & 201 & 403 & 571 & 564 & 219 \\ 592 & 334 & 402 & 746 & 491 & 228 \\ 646 & 257 & 667 & 357 & 328 & 229 \\ 477 & 223 & 417 & 626 & 308 & 122 \\ \mathbf{5 3 7 . 6} & \mathbf{2 5 8 . 2} & \mathbf{4 8 3} & \mathbf{5 9 1} & \mathbf{4 8 7 . 8} & \mathbf{2 0 7 . 4} \\ \text { FR10 } & & & & & \\ 414 & 110 & 360 & 320 & 705 & 187 \\ 516 & 286 & 646 & 469 & 769 & 244 \\ 654 & 156 & 575 & 448 & 727 & 243 \\ \mathbf{7 9 1} & 281 & 260 & 59 & 885 & 352 \\ 544 & 199 & 856 & 378 & 695 & 200 \\ \mathbf{5 8 3 . 8} & \mathbf{2 0 6 . 4} & \mathbf{5 3 9 . 4} & \mathbf{3 3 4 . 8} & \mathbf{7 5 6 . 2} & \mathbf{2 4 5 . 2} \\ \text { FR5 } & & & & & \\ 1095 & 563 & & 456 & 592 & 444 \\ 972 & 456 & & 538 & 872 & 378 \\ \mathbf{8 7 8} & \mathbf{6 6 7} & & 606 & 683 & 617 \\ 282 & 503 & & 576 & 909 & 332 \\ 485 & 552 & & 815 & 992 & 575\end{array}$


APPENDIX 3.1

Time Spent Contacting or Cohabiting with Objects (Out of 50) from Experiment 3.1 (Relating to Figures 3.2 and 3.3)

Number of Ten Second point Samples During Which the Rats Were in Nose or Mouth Contact with a Object.

Two objects changed daily

$\begin{array}{llllllllll}14 & 1 & 8 & 1 & 12 & 0 & 15 & 0 & 21 & 0 \\ 11 & 1 & 5 & 1 & 19 & 0 & 7 & 1 & 21 & 1 \\ 16 & 0 & 15 & 0 & 16 & 0 & 22 & 0 & 9 & 0 \\ 14 & 0 & 18 & 0 & 6 & 0 & 20 & 1 & 13 & 0 \\ 10 & 0 & 5 & 0 & 9 & 1 & 4 & 0 & 14 & 2 \\ 9 & 1 & 4 & 0 & 5 & 3 & 1 & 0 & 11 & 2 \\ 3 & 0 & 20 & 1 & 3 & 0 & 4 & 1 & 19 & 0 \\ 1 & 0 & 10 & 0 & 6 & 1 & 5 & 0 & 12 & 0\end{array}$

Two objects unchanged

$\begin{array}{llllllllll}14 & 1 & 10 & 1 & 2 & 4 & 9 & 0 & 7 & 1 \\ 17 & 1 & 4 & 1 & 1 & 2 & 1 & 1 & 3 & 3 \\ 12 & 0 & 0 & 0 & 0 & 0 & 0 & 0 & 0 & 0 \\ 17 & 1 & 2 & 1 & 2 & 0 & 2 & 0 & 0 & 1 \\ 3 & 0 & 0 & 0 & 1 & 0 & 1 & 0 & 0 & 0 \\ 10 & 1 & 1 & 4 & 1 & 5 & 0 & 0 & 1 & 3 \\ 6 & 2 & 2 & 0 & 3 & 0 & 0 & 5 & 1 & 0 \\ 1 & 0 & 0 & 0 & 15 & 1 & 2 & 0 & 3 & 0\end{array}$

Four objects changed daily

$\begin{array}{llllllllll}18 & 1 & 22 & 0 & 17 & 1 & 20 & 0 & 7 & 4 \\ 17 & 2 & 9 & 1 & 9 & 2 & 15 & 0 & 14 & 5 \\ 12 & 1 & 13 & 1 & 17 & 2 & 10 & 0 & 10 & 4 \\ 17 & 1 & 20 & 1 & 13 & 0 & 12 & 0 & 12 & 0 \\ 7 & 0 & 3 & 2 & 8 & 2 & 16 & 24 & 7 & 0 \\ 10 & 1 & 5 & 1 & 23 & 2 & 26 & 19 & 6 & 1 \\ 18 & 0 & 20 & 1 & 19 & 5 & 24 & 2 & 19 & 8 \\ 17 & 1 & 21 & 2 & 17 & 1 & 30 & 1 & 7 & 1\end{array}$

Four objects unchanged

$\begin{array}{llllllllll}15 & 0 & 0 & 0 & 0 & 1 & 4 & 1 & 3 & 2 \\ 8 & 0 & 1 & 2 & 2 & 0 & 3 & 0 & 0 & 0 \\ 24 & 1 & 1 & 2 & 0 & 1 & 1 & 0 & 1 & 4\end{array}$




$\begin{array}{llllllllll}9 & 1 & 5 & 1 & 1 & 3 & 0 & 0 & 0 & 1 \\ 15 & 3 & 3 & 2 & 1 & 9 & 3 & 3 & 3 & 3 \\ 23 & 3 & 5 & 2 & 2 & 1 & 4 & 3 & 6 & 5 \\ 11 & 1 & 1 & 1 & 2 & 2 & 1 & 5 & 14 & 3 \\ 9 & 0 & 2 & 0 & 4 & 2 & 1 & 13 & 2 & 0\end{array}$

Number of Ten Second Point Samples During Which the Rats Were on the Same Side of the Cage as the Objects.

Two objects changed daily

$\begin{array}{llllllllll}14 & 17 & 22 & 15 & 29 & 10 & 22 & 21 & 26 & 17 \\ 13 & 17 & 16 & 19 & 29 & 16 & 21 & 19 & 27 & 19 \\ 13 & 18 & 11 & 14 & 12 & 13 & 13 & 18 & 15 & 3 \\ 20 & 16 & 24 & 23 & 23 & 8 & 7 & 14 & 9 & 8 \\ 7 & 16 & 15 & 14 & 18 & 8 & 19 & 1 & 8 & 11 \\ 7 & 19 & 23 & 22 & 8 & 20 & 19 & 10 & 12 & 18 \\ 23 & 28 & 25 & 28 & 24 & 20 & 25 & 21 & 9 & 16 \\ 26 & 26 & 23 & 26 & 17 & 21 & 23 & 23 & 21 & 23\end{array}$

Two objects unchanged

$\begin{array}{llllllllll}16 & 24 & 10 & 18 & 4 & 17 & 5 & 20 & 16 & 10 \\ 11 & 12 & 7 & 22 & 14 & 18 & 11 & 17 & 19 & 6 \\ 13 & 20 & 8 & 13 & 18 & 8 & 21 & 3 & 18 & 7 \\ 8 & 16 & 7 & 12 & 12 & 10 & 19 & 8 & 13 & 3 \\ 8 & 15 & 14 & 13 & 11 & 10 & 16 & 18 & 11 & 10 \\ 14 & 25 & 18 & 19 & 18 & 18 & 14 & 22 & 9 & 17 \\ 14 & 22 & 23 & 23 & 22 & 26 & 25 & 26 & 12 & 24 \\ 14 & 23 & 28 & 18 & 11 & 29 & 19 & 30 & 10 & 27\end{array}$

Four objects changed daily

$\begin{array}{llllllllll}24 & 16 & 27 & 18 & 25 & 13 & 24 & 16 & 25 & 21 \\ 22 & 15 & 24 & 26 & 21 & 13 & 24 & 18 & 23 & 20 \\ 10 & 27 & 8 & 23 & 4 & 14 & 22 & 26 & 21 & 7 \\ 12 & 25 & 10 & 18 & 10 & 18 & 23 & 28 & 5 & 16 \\ 12 & 15 & 15 & 15 & 7 & 22 & 18 & 14 & 13 & 12 \\ 9 & 19 & 3 & 19 & 11 & 19 & 9 & 12 & 17 & 11 \\ 23 & 19 & 17 & 10 & 10 & 15 & 13 & 17 & 17 & 21 \\ 8 & 21 & 12 & 10 & 9 & 17 & 18 & 15 & 14 & 24\end{array}$

Four objects unchanged 


$\begin{array}{llllllllll}16 & 16 & 11 & 10 & 12 & 16 & 10 & 9 & 8 & 15 \\ 15 & 22 & 15 & 15 & 4 & 19 & 7 & 21 & 10 & 15 \\ 6 & 19 & 14 & 17 & 20 & 6 & 28 & 15 & 12 & 10 \\ 5 & 15 & 13 & 16 & 16 & 13 & 13 & 17 & 12 & 22 \\ 19 & 13 & 20 & 22 & 16 & 17 & 11 & 14 & 6 & 21 \\ 15 & 14 & 12 & 19 & 15 & 13 & 23 & 17 & 10 & 20 \\ 5 & 26 & 20 & 24 & 7 & 24 & 10 & 13 & 15 & 14 \\ 9 & 26 & 19 & 21 & 8 & 30 & 18 & 25 & 16 & 24\end{array}$

control

$\begin{array}{llllllllll}24 & 24 & 10 & 29 & 17 & 12 & 10 & 14 & 0 & 29 \\ 1 & 21 & 0 & 8 & 0 & 4 & 30 & 10 & 0 & 9 \\ 2 & 12 & 11 & 29 & 20 & 26 & 7 & 14 & 5 & 25 \\ 14 & 26 & 2 & 29 & 3 & 14 & 11 & 8 & 14 & 23 \\ 2 & 26 & 1 & 27 & 5 & 16 & 5 & 14 & 0 & 11 \\ 3 & 21 & 0 & 19 & 4 & 20 & 9 & 10 & 0 & 30 \\ 13 & 19 & 9 & 13 & 2 & 8 & 1 & 5 & 0 & 7 \\ 2 & 19 & 0 & 29 & 0 & 1 & 0 & 2 & 1 & 2\end{array}$


APPENDIX 3.2

Choice for the Non-Standard Option (Out of 60) from Experiment 3.2 (Relating to Figure 3.3)

\begin{tabular}{|c|c|c|c|c|c|}
\hline & $\begin{array}{l}\text { rat } \\
\text { no barriers }\end{array}$ & Space & Objects & Rats & Pillars \\
\hline Female1 (Cont.) & 40 & 47 & 21 & 40 & 31 \\
\hline Female1 (T) & 45 & 35 & 22 & 40 & 31 \\
\hline Female2 (Cont.) & 40 & 50 & 30 & 19 & 28 \\
\hline Female2 $(\mathrm{T})$ & 31 & 42 & 28 & 36 & 39 \\
\hline Female3 (Cont.) & 38 & 38 & 36 & 38 & 34 \\
\hline Female3 (T.) & 34 & 35 & 34 & 34 & 36 \\
\hline Female4 (Cont.) & 30 & 24 & 28 & 40 & 28 \\
\hline Female4 (T) & 32 & 39 & 35 & 37 & 38 \\
\hline Female5 (Cont.) & 45 & 45 & 34 & 28 & 32 \\
\hline Female5 (T) & 29 & 31 & 33 & 42 & 43 \\
\hline Female6 (Cont.) & 31 & 30 & 28 & 30 & 37 \\
\hline Female6 (T) & 28 & 32 & 31 & 34 & 30 \\
\hline Male 1 (Cont.) & & 16 & 12 & 41 & 14 \\
\hline Malel $(\mathrm{T})$ & & 40 & 28 & 35 & 22 \\
\hline Male2 (Cont.) & & 47 & 17 & 12 & 18 \\
\hline Male2 (T) & & 35 & 29 & 41 & 34 \\
\hline Male3 (Cont.) & & 51 & 35 & 23 & 29 \\
\hline Male3 (T) & & 27 & 26 & 34 & 30 \\
\hline Male4 (Cont.) & & 33 & 14 & 24 & 28 \\
\hline Male4 (T) & & 30 & 32 & 29 & 26 \\
\hline Male5 (Cont.) & & 47 & 18 & 40 & 52 \\
\hline $\operatorname{Male5}(\mathrm{T})$ & & 32 & 28 & 43 & 36 \\
\hline Male6 (Cont.) & & 43 & 34 & 19 & 27 \\
\hline Male6 (T) & & 28 & 28 & 36 & 27 \\
\hline
\end{tabular}




\section{APPENDIX 3.3}

Choice for the Non-Standard Option (Out of 60) from Experiment 3.3 (Relating to Figure 3.4, 3.5 and 3.6)

tunnel 3-tunnel platform plastic box Shredded tin box sticks tissue walnuts

\begin{tabular}{|c|c|c|c|c|c|c|c|c|c|}
\hline $\begin{array}{l}\text { Female1 } \\
\text { (Cont.) }\end{array}$ & 22 & $26 / 2 / 17$ & 46 & 30 & $39 / 2 / 0$ & $38 / 15 / 6$ & 21 & 40 & $29 / 0$ \\
\hline $\begin{array}{l}\text { Female 1 } \\
\text { (T) }\end{array}$ & 30 & $34 / 4 / 5$ & 40 & 36 & $28 / 0 / 0$ & $34 / 2 / 5$ & 37 & 35 & $33 / 0$ \\
\hline $\begin{array}{l}\text { Female2 } \\
\text { (Cont.) }\end{array}$ & 34 & $49 / 17 / 0$ & 40 & 29 & $49 / 14 / 26$ & 54 & 35 & 41 & $44 / 2$ \\
\hline $\begin{array}{l}\text { Female2 } \\
\text { (T) }\end{array}$ & 31 & $43 / 7 / 1$ & 36 & 30 & $38 / 2 / 24$ & $41 / 18 / 0$ & 33 & 29 & $32 / 0$ \\
\hline $\begin{array}{l}\text { Female3 } \\
\text { (Cont.) }\end{array}$ & 25 & $36 / 2 / 6$ & 23 & 31 & $48 / 2 / 30$ & $52 / 42 / 3$ & 31 & 48 & $32 / 3$ \\
\hline $\begin{array}{l}\text { Female3 } \\
\text { (T.) }\end{array}$ & 34 & $39 / 4 / 2$ & 34 & 32 & $32 / 0 / 13$ & $45 / 28 / 2$ & 31 & 31 & $35 / 3$ \\
\hline $\begin{array}{l}\text { Female4 } \\
\text { (Cont.) }\end{array}$ & 34 & $40 / 3 / 13$ & 25 & 24 & $53 / 6 / 9$ & $59 / 57 / 0$ & 34 & 44 & $23 / 1$ \\
\hline $\begin{array}{l}\text { Female4 } \\
\text { (T) }\end{array}$ & 23 & $27 / 0 / 1$ & 38 & 28 & $37 / 0 / 0$ & $47 / 45 / 0$ & 33 & 29 & $28 / 0$ \\
\hline $\begin{array}{l}\text { Female5 } \\
\text { (Cont.) }\end{array}$ & 23 & $47 / 2 / 18$ & 48 & 31 & $50 / 8 / 13$ & $35 / 24 / 0$ & 31 & 55 & $31 / 7$ \\
\hline $\begin{array}{l}\text { Female } 5 \\
\text { (T) }\end{array}$ & 27 & $32 / 4 / 1$ & 43 & 33 & $31 / 1 / 4$ & $38 / 15 / 1$ & 27 & 34 & $27 / 1$ \\
\hline $\begin{array}{l}\text { Female6 } \\
\text { (Cont.) }\end{array}$ & 21 & $19 / 7 / 3$ & 39 & 26 & $46 / 20 / 4$ & $37 / 19 / 1$ & 31 & 41 & $29 / 3$ \\
\hline $\begin{array}{l}\text { Female6 } \\
\text { (T) }\end{array}$ & 28 & $36 / 0 / 0$ & 38 & 37 & $33 / 1 / 3$ & $32 / 7 / 2$ & 34 & 29 & $28 / 2$ \\
\hline $\begin{array}{l}\text { Male1 } \\
\text { (Cont.) }\end{array}$ & 40 & $38 / 5 / 6$ & 17 & 40 & $37 / 2 / 0$ & $42 / 2 / 20$ & 29 & 34 & $22 / 0$ \\
\hline Male1 (T) & 30 & $37 / 0 / 1$ & 44 & 26 & $38 / 3 / 0$ & $31 / 0 / 2$ & 31 & 37 & $20 / 0$ \\
\hline $\begin{array}{l}\text { Male2 } \\
\text { (Cont.) }\end{array}$ & 37 & $40 / 10 / 1$ & 42 & 34 & $59 / 16 / 39$ & $42 / 31 / 0$ & 24 & 42 & $34 / 0$ \\
\hline Male2 (T) & 31 & $35 / 4 / 0$ & 36 & 38 & $52 / 2 / 45$ & $45 / 35 / 0$ & 34 & 38 & $34 / 0$ \\
\hline $\begin{array}{l}\text { Male3 } \\
\text { (Cont.) }\end{array}$ & 37 & $20 / 0 / 0$ & 36 & 11 & $54 / 15 / 28$ & $41 / 19 / 1$ & 17 & 31 & $37 / 8$ \\
\hline Male3 (T) & 35 & $28 / 1 / 0$ & 33 & 28 & $48 / 1 / 44$ & $35 / 28 / 0$ & 28 & 32 & $28 / 0$ \\
\hline $\begin{array}{l}\text { Male4 } \\
\text { (Cont.) }\end{array}$ & 33 & $43 / 5 / 17$ & 32 & 18 & $45 / 2 / 10$ & $22 / 0 / 0$ & 26 & 32 & $27 / 0$ \\
\hline Male4 $(\mathrm{T})$ & 25 & $38 / 4 / 10$ & 30 & 28 & $35 / 0 / 7$ & $27 / 0 / 0$ & 25 & 38 & $33 / 5$ \\
\hline Male5 & 30 & $32 / 1 / 7$ & 20 & 26 & $39 / 3 / 18$ & $34 / 22 / 1$ & 31 & 26 & $32 / 9$ \\
\hline
\end{tabular}


(Cont.)

$\begin{array}{lccccccccc}\text { Male5 (T) } & 29 & 24 / 1 / 0 & 36 & 27 & 29 / 0 / 13 & 56 / 43 / 3 & 28 & 35 & 27 / 0 \\ \begin{array}{l}\text { Male6 } \\ \text { (Cont.) }\end{array} & 19 & 31 / 3 / 2 & 44 & 41 & 24 / 0 / 5 & 49 / 1 / 0 & 30 & 35 & 25 / 3 \\ \text { Male6 (T) } & 20 & 29 / 0 / 0 & 44 & 36 & 34 / 0 / 3 & 35 / 1 / 0 & 30 & 30 & 32 / 0\end{array}$

single tunnel C $\quad 29.58333 \quad 2.090593$

$\begin{array}{lll}\mathrm{T} & 28.58333 & 1.252019\end{array}$

3-tunnel $\quad \mathrm{C} \quad 35.08333 \quad 2.821611$

$\begin{array}{lll}\mathrm{T} & 33.5 & 1.621354\end{array}$

$\begin{array}{llll}\text { platform } & \mathrm{C} & 34.33333 & 3.087939\end{array}$

$\begin{array}{lll}\mathrm{T} & \mathbf{3 7 . 6 6 6 6 7} & 1.28117\end{array}$

$\begin{array}{llll}\text { container } & \text { C } & 28.41667 & 2.435403\end{array}$

$\begin{array}{lll}\mathrm{T} & 31.5833 & 1.245953\end{array}$

$\begin{array}{llll}\text { nest box } & C & \mathbf{4 2 . 0 8 3 3 3} & 2.934292\end{array}$

$\begin{array}{lll}T & \mathbf{3 8 . 8 3 3 3 3} & 2.373571\end{array}$

$\begin{array}{llll}\text { sticks } & \text { C } & 28.33333 & 5.348464\end{array}$

$\begin{array}{lll}\text { T } & 30.9187 & 3.476109\end{array}$

$\begin{array}{llll}\text { walnuts } & \mathrm{C} & 30.41667 & 6.15642\end{array}$

$\begin{array}{lll}\mathrm{T} & 29.75 & 4.22385\end{array}$

$\begin{array}{llll}\text { tissue paper } & \mathrm{C} & 39.08333 & 2.307024\end{array}$

$\begin{array}{lll}\mathrm{T} & 33.08333 & 1.018453\end{array}$

$\begin{array}{llll}\text { shredded } & C & \mathbf{4 5 . 2 5} & 2.708363\end{array}$

$\begin{array}{llll}\text { paper } & \mathrm{T} & \mathbf{3 6 . 2 5} & 2.089385\end{array}$ 


\section{APPENDIX 4.1}

Parameters of Individual Demand Curves (Relating to Figure 4.1-4.3)

The fitted lines and their parameters are based on Hursh, Raslear, Shurtleff, Bauman \& Simmons, 1988a) referred to in the text as Equation 4.0. A '*' is an invalid data point which counts as 0.0 in any calculation, missing data is indicated as NA.

\section{$p \max$}

$\begin{array}{lllllllllllll} & \text { A } & \text { A } & \text { A } & \text { B } & \text { B } & \text { B } & \text { A } & \text { A } & \text { A } & \text { C } & \text { C } & \text { C } \\ \text { rat1 } & 8.495 & 8.069 & * & 9.069 & 7.91 & 8.70 & 3.83 & 1.02 & 9.81 & 5.25 & 1.26 & 5.10 \\ \text { rat2 } & 14.07 & 8.502 & 5.915 & 10.36 & 14.15 & 19.74 & 15.95 & 14.91 & \text { NA } & 5.06 & 5.97 & 2.76 \\ \text { rat3 } & 12.09 & 7.052 & 10.54 & 9.97 & 10.37 & 8.88 & 6.11 & 11.43 & 8.14 & \text { NA } & \text { NA } & \text { NA } \\ \text { rat4 } & 9.048 & 7.821 & 9.758 & 38.41 & 18.07 & 20.07 & 7.81 & 13.05 & 5.05 & 6.94 & 11.97 & 2.05 \\ \text { rat5 } & 12.87 & 11.32 & 11.68 & 16.3 & 7.76 & 10.56 & 5.59 & 4.98 & 6.68 & \text { NA } & \text { NA } & \text { NA } \\ \text { rat6 } & * & 13.64 & 12.13 & 14.02 & 7.02 & 15.68 & 6.76 & 10.98 & 5.45 & 6.90 & 3.82 & 4.41\end{array}$

$a$ values

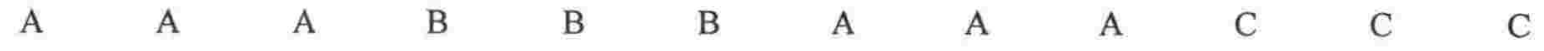

$\begin{array}{lllllllllllll}1 & 0.072 & 0.120 & 0.029 & 0.043 & 0.034 & 0.065 & 0.244 & 0.071 & 0.070 & 0.255 & 0.301 & 0.620\end{array}$

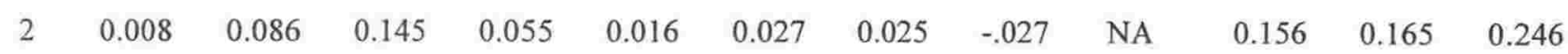

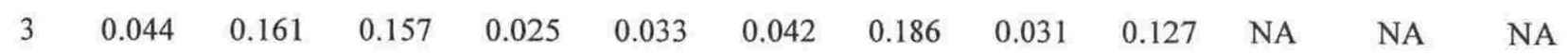

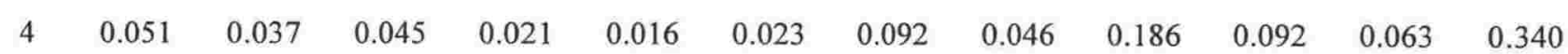

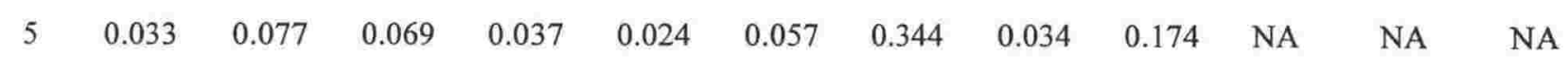

$\begin{array}{lllllllllllll}6 & 0.005 & 0.042 & 0.044 & 0.039 & 0.012 & 0.029 & 0.146 & 0.091 & 0.191 & -.157 & 0.251 & 0.059\end{array}$

$b$ values

$\begin{array}{lllllllllllll} & \mathrm{A} & \mathrm{A} & \mathrm{A} & \mathrm{B} & \mathrm{B} & \mathrm{B} & \mathrm{A} & \mathrm{A} & \mathrm{A} & \mathrm{C} & \mathrm{C} & \mathrm{C} \\ 1 & 0.412 & 1.223 & -.980 & -.109 & 0.184 & 0.302 & 1.153 & -.834 & 0.581 & 2.085 & -.129 & 6.286 \\ 2 & -.749 & 0.683 & 0.969 & 0.313 & -.120 & 0.227 & -.082 & -1.927 & \mathrm{NA} & 0.816 & 1.268 & 0.565 \\ 3 & 0.231 & 1.622 & 2.825 & -.426 & 0.003 & -.141 & 1.618 & -.184 & 1.379 & \mathrm{NA} & \mathrm{NA} & \mathrm{NA} \\ 4 & 0.070 & -.341 & 0.989 & 0.856 & -.043 & 0.063 & 0.654 & 0.382 & 1.162 & 0.660 & .737 & 0.603 \\ 5 & -.012 & 1.005 & 1.067 & 0.389 & 0.018 & 0.414 & 3.430 & -.610 & 1.677 & \text { NA } & \text { NA } & \text { NA } \\ 6 & -1.18 & 0.319 & 0.229 & 0.259 & -.531 & 0.047 & 1.273 & 1.301 & 1.396 & -3.496 & 1.205 & -.401\end{array}$

$L$ values

S A

A $A$ B

B

B

A

A

A

C

C $\mathrm{C}$ 
$\begin{array}{llllllllllllll}1 & 282.7 & 274.2 & * & 824.6 & 409.6 & 677.6 & 431.5 & 1234.5 & 202.3 & 79.8 & 1594 & 2.4\end{array}$

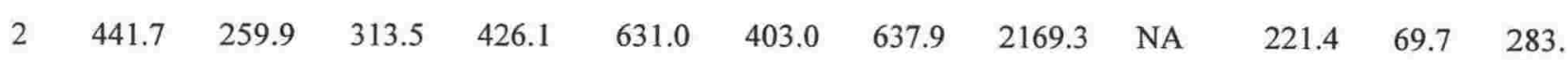

$\begin{array}{lllllllllllll}3 & 469.4 & 221.0 & 7.973 & 1053.9 & 584.8 & 692.4 & 154.1 & 633.9 & 162.5 & \text { NA } & \text { NA } & \text { NA }\end{array}$

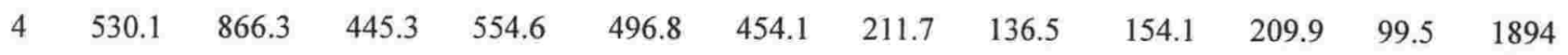

$\begin{array}{lllllllllllll}5 & 583.7 & 154.7 & 128.9 & 362.2 & 412.4 & 469.6 & 77.8 & 677.3 & 188.1 & \text { NA } & \text { NA } & \text { NA }\end{array}$

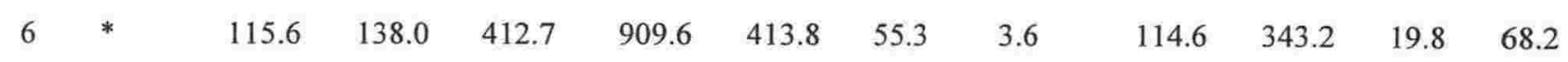




\section{APPENDIX 4.2}

Parameters of Individual Demand Curves (Relating to Figure 4.4 and 4.5, and Table 4.2.1 and 4.2.2)

Response rate at the lowest FR (2)

\begin{tabular}{lllllll} 
& & \multicolumn{5}{c}{ Reinforcers Obtained at FR2 } \\
& Subject 1 & & Subject 2 & \multicolumn{3}{c}{ Subject3 } \\
standard & 17 & 18 & 15 & 17 & 18 & 13 \\
space & 16 & 18 & 16 & 15 & 15 & 15 \\
3 rats & 17 & & 15 & 16 & 14 & 5 \\
objects & 15 & 13 & 10 & 14 & 15 & 13 \\
chocolate & 17 & 15 & 17 & 16 & 18 & 16 \\
pillars & 11 & & 2 & 15 & 14 & 15
\end{tabular}

\begin{tabular}{lllllll} 
& & & \multicolumn{5}{c}{ Last FR obtained } \\
& Subject 1 & & Subject 2 & \multicolumn{4}{c}{ Subject3 } \\
standard & 72 & 24 & 44 & 20 & 80 & 54 \\
space & 42 & 46 & 42 & 54 & 28 & 78 \\
3 rats & 50 & 58 & 56 & 78 & 108 & 88 \\
objects & 30 & 26 & 20 & 24 & 54 & 30 \\
chocolate & 24 & 20 & 8 & 14 & 14 & 16 \\
pillars & 36 & 28 & 20 & 24 & 80 & 76
\end{tabular}

pmax

\begin{tabular}{llllllllll} 
& standard & \multicolumn{9}{c}{ Space } & \multicolumn{7}{c}{ Rats } \\
& 1 & 2 & 3 & 1 & 2 & 3 & 1 & 2 & 3 \\
rat1 & 37.4 & 10.7 & 35.0 & 2.7 & 12.4 & 15.3 & 11.3 & 31.4 & 31.3 \\
rat2 & 25.6 & 9.8 & 38.8 & 23.8 & 28.3 & 26.5 & 95.5 & 44.4 & 66.2 \\
rat3 & 37.1 & 60.3 & 19.4 & 80.4 & 25.9 & 56.2 & 730.9 & 120.9 & 54.7 \\
& Objects & & & Pillars & & & Semi- & & \\
& & & & & & & Enriched & & \\
& 1 & 2 & 3 & 1 & 2 & 3 & 1 & 2 & 3 \\
rat1 & 77.2 & 17.4 & 35.0 & 55.8 & 43.3 & 52.0 & 60.7 & 58.3 & 18.1 \\
rat2 & 55.0 & 95.5 & 42.8 & 35.2 & $*$ & 12.6 & 23.2 & 34.7 & 45.3 \\
rat3 & 66.2 & 35.8 & 151.8 & 26.6 & 27.5 & 33.6 & 193.6 & 20.1 & 38.5
\end{tabular}

$a$ values

\begin{tabular}{llllllllll} 
& standard & \multicolumn{9}{c}{ Space } & \multicolumn{7}{c}{ Rats } \\
& 1 & 2 & 3 & 1 & 2 & 3 & 1 & 2 & 3 \\
rat1 & .009 & .079 & .013 & .005 & .063 & .032 & .063 & .014 & .017 \\
rat2 & .021 & .054 & .012 & .025 & .019 & .025 & .003 & .009 & .007 \\
rat3 & .014 & .006 & .019 & .005 & .021 & .008 & .001 & .002 & .007 \\
& Objects & & & Pillars & & & Semi- & & \\
& & & & & & & Enriched & & \\
& 1 & 2 & 3 & 1 & 2 & 3 & 1 & 2 & 3
\end{tabular}




$\begin{array}{llllllllll}\text { rat1 } & .009 & .030 & .013 & .008 & .002 & .005 & .007 & .005 & .034 \\ \text { rat2 } & .011 & .003 & .010 & .017 & -.002 & .065 & .024 & .020 & .010 \\ \text { rat3 } & .007 & .011 & .002 & .016 & .012 & .013 & .002 & .025 & .010\end{array}$

$$
b \text { values }
$$

standard

$\begin{array}{ll}\text { rat1 } & -.209 \\ \text { rat2 } & .221 \\ \text { rat3 } & .183\end{array}$

Objects

rat1 -.335

rat2 .404

rat3 -.007

\section{Space}

23

$.941 \quad .06$

$.206 \quad .109$

$-.199 \quad-.171$

Pillars

23

.206

1

$-.136$

.258

.244

.537

.537

.023

3

Rats

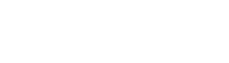

standard

$$
\text { rat }
$$

$$
\text { rat3 }
$$

standar

$\begin{array}{lll} & \text { Space } \\ 2 & 3 & 1\end{array}$

\section{$L$ values}

$\begin{array}{lllll} & 1 & 2 & 3 & 1 \\ \text { rat1 } & 13.52 & 10.78 & 10.18 & 12.00\end{array}$

rat2 $\quad 10.92 \quad 19.66 \quad 6.31 \quad 15.41$

rat3 14.92

8.85

16.18

14.11

23

Rats

Objects

Pillars

$\begin{array}{lll}5.79 & 15.24 & 11.34\end{array}$

23

\section{Enriched}

$1 \quad 2 \quad 3$




\section{APPENDIX 4.3}

Parameters of Individual Demand Curves (Relating to Figure 4.6)

\begin{tabular}{|c|c|c|c|c|c|c|c|c|c|}
\hline & & & & & $\operatorname{nax}$ & & & & \\
\hline & 1 rats & & & 2 rat & & & 4 rats & & \\
\hline & 1 & 2 & 3 & 1 & 2 & 3 & 1 & 2 & 3 \\
\hline rat1 & 27.3307 & 43.1855 & 53.8176 & 33.5075 & 45.3535 & 25.1206 & 705.4561 & 41.8916 & 8.2030 \\
\hline rat2 & 20.7411 & 128.4627 & 15.7849 & 23.7993 & 46.0117 & 23.7443 & 93.0384 & 10.7265 & 44.5993 \\
\hline rat3 & 39.8883 & 17.8883 & 28.9895 & 110.0533 & 80.3319 & $?$ & 45.6743 & 80.5045 & $?$ \\
\hline rat4 & 10.3065 & 37.2742 & 50.3817 & 34.9302 & 42.6386 & 13.4961 & 18.9897 & 10.7514 & 6.7339 \\
\hline & 6 rat & & & 12 rat & & & Chocolate & & \\
\hline & 1 & 2 & 3 & 1 & 2 & 3 & 1 & 2 & 3 \\
\hline rat1 & 43.0812 & 39.4581 & 104.7577 & 30.1321 & 31.4289 & 59.9442 & 30.8488 & 30.5914 & 36.7596 \\
\hline rat2 & 29.6004 & 63.9121 & 56.3350 & 29.0065 & 104.385 & 79.8666 & 41.7061 & 52.4394 & 41.9089 \\
\hline rat3 & 67.1483 & 52.9517 & 75.0428 & 33.5805 & 33.4300 & 59.7093 & 37.3264 & 62.9922 & 151.1165 \\
\hline rat4 & 22.8045 & $\begin{array}{l}810.845 \\
3\end{array}$ & 76.4420 & 28.9180 & 27.1828 & 23.0845 & 32.2742 & 24.1369 & 33.5281 \\
\hline
\end{tabular}

$a$ values

1 rats

2 rat

23

1

rat

1

$$
\text { rat2 }
$$

rat3

rat 4

$\begin{array}{llll} & .01229 & .02935 & .00858 \\ \text { rat4 } & .05962 & .00913 & .00603\end{array}$

6 rat

1

rat rat

$\begin{array}{llllllllll}\text { at4 } & .0185 & .00025 & .00418 & .0115 & .0117 & .0146 & .01258 & .01987 & .01421\end{array}$

$b$ values

\begin{tabular}{llllllllll}
\multicolumn{1}{c}{1 rats } & \multicolumn{9}{c}{2 rat } \\
& 1 & 2 & 3 & 1 & 2 & 3 & 1 & 2 & 3 \\
rat1 & .12332 & -.23532 & -.10778 & -.13202 & -.27943 & -.00974 & -.47963 & -.2727 & -.47963 \\
rat2 & .20685 & .11015 & .34689 & .4933 & -.23931 & -.05251 & -.29733 & .26186 & .05261 \\
rat3 & .12879 & .20879 & .28031 & -.4273 & -.10844 & $*$ & -.02298 & -.16584 & $?$
\end{tabular}




\begin{tabular}{|c|c|c|c|c|c|c|c|c|c|}
\hline \multirow[t]{2}{*}{ rat4 } & .47473 & -.2164 & -.30047 & -.25522 & -.22144 & -.20881 & .59948 & .1467 & -.31617 \\
\hline & \multicolumn{3}{|l|}{6 rats } & \multicolumn{3}{|l|}{12 rats } & \multicolumn{3}{|l|}{ Chocolate } \\
\hline & 1 & 2 & 3 & 1 & 2 & 3 & 1 & 2 & 3 \\
\hline rat1 & -.15384 & -.08781 & -.28842 & -.11816 & .07683 & -.16494 & .11094 & .02003 & -.13919 \\
\hline rat2 & .20434 & -.18766 & -.09222 & -.03266 & -.29311 & -.14119 & -.06465 & -.09078 & -.15467 \\
\hline rat3 & -.10942 & .09977 & -.0842 & .19849 & 16.7744 & -.0321 & 23.32389 & .26189 & .09607 \\
\hline rat4 & -.02858 & -.53324 & -.26426 & -.23171 & -.1046 & -.22395 & -.06513 & .10432 & .09703 \\
\hline \multicolumn{10}{|c|}{$L$ values } \\
\hline & 1 rats & & & 2 rat & & & 4 rats & & \\
\hline & 1 & 2 & 3 & 1 & 2 & 3 & 1 & 2 & 3 \\
\hline rat1 & 12.91198 & 10.10887 & 8.88668 & 13.60681 & 17.12499 & 15.40559 & 16.01234 & 16.40679 & 10.64781 \\
\hline rat2 & 14.95854 & 3.20265 & 15.51115 & 10.77336 & 17.70954 & 16.67888 & 16.56447 & 15.73028 & 6.64503 \\
\hline rat3 & 10.77176 & 13.61608 & 7.40321 & 15.03633 & 10.63084 & $*$ & 16.25149 & 12.53906 & $?$ \\
\hline \multirow[t]{3}{*}{ rat4 } & 16.72891 & 11.16145 & 14.81982 & 18.50146 & 16.72176 & 12.70917 & 7.59457 & 18.58366 & 15.06637 \\
\hline & 6 rats & & & 12 rats & & & Chocolate & & \\
\hline & 1 & 2 & 3 & 1 & 2 & 3 & 1 & 2 & 3 \\
\hline rat1 & 16.75782 & 14.35552 & 15.33255 & 15.29761 & 7.74121 & 11.86576 & 18.48617 & 18.46492 & 17.17034 \\
\hline rat2 & 15.41958 & 14.65914 & 8.37642 & 15.40682 & 15.77613 & 10.20626 & 19.54069 & 19.30552 & 20.93986 \\
\hline rat3 & 13.15918 & 9.83309 & 10.81853 & 9.04901 & 0 & 9.43269 & 0 & 9.86358 & 12.75795 \\
\hline rat4 & 16.78341 & 13.16185 & 10.38981 & 16.20117 & 15.30829 & 11.98478 & 18.33745 & 15.88777 & 12.18948 \\
\hline
\end{tabular}

